$D O E / I D / 13223-78$

\title{
A GEOTHERMAL RESOURCE DATA BASE NEW MEXICO
}

\author{
James C. Witcher
}

Southwest Technology Development Institute

New Mexico State University

Box 30001 Dept 3SOL

Las Cruces, New Mexico 88003

\author{
JULY 1995
}




\section{DISCLAIMER}

This report was prepared as an account of work sponsored by the United States Government. Neither the United States nor any agency thereof, nor any of their employees, makes any warranty, expressed or implied, or assumes any legal liability or responsibility for the accuracy, completeness, or usefulness of any information, apparatus, product, or process disclosed, or represents that its use would not infringe privately owned rights. Reference to any specific commercial product, process, or service by trade name, trademark, manufactururer, or otherwise, does not constitute or imply its endorsement, recommendation, or favoring by the Southwest Technology Development Institute, New Mexico State University, United States Government or any agency thereof. The views and opinions of the author expressed herein do not necessarily state or reflect those of the United States Government or any agency thereof. 


\section{TABLE OF CONTENTS}

INTRODUCTION

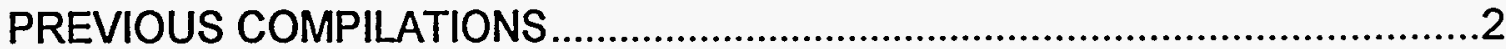

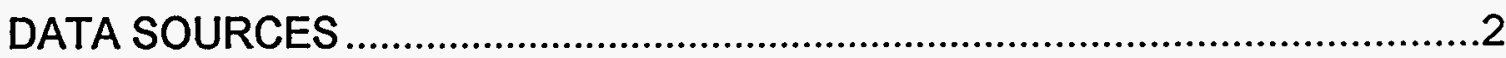

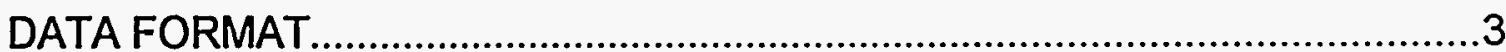

OVERVIEW OF DATABASE

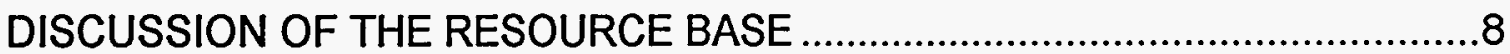

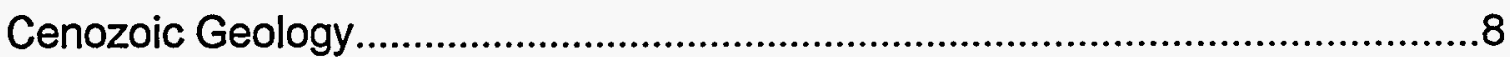

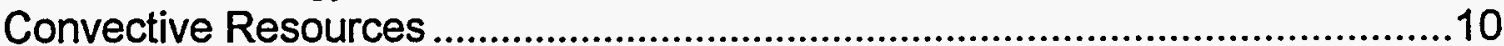

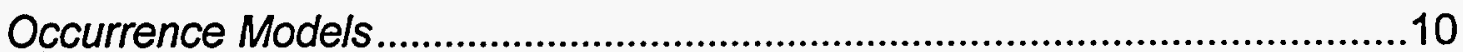

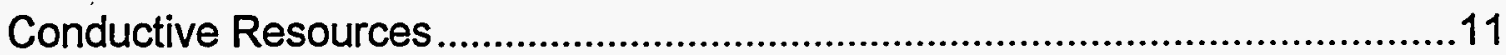

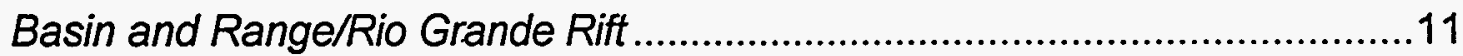

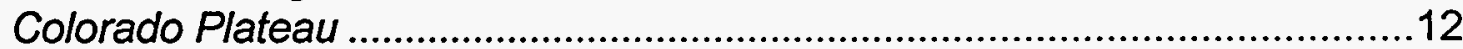

PRIORITY AREAS AND AREAS WITH NEAR-TERM POTENTIAL .................12

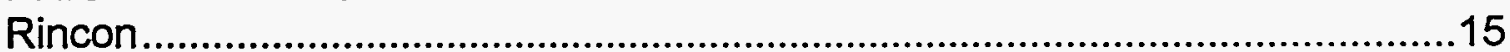

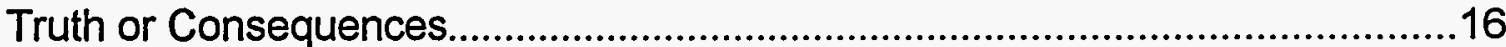

Las Cruces East Mesa/Tortugas Mountain ......................................................18

Montezuma Hot Springs/United World College .............................................19

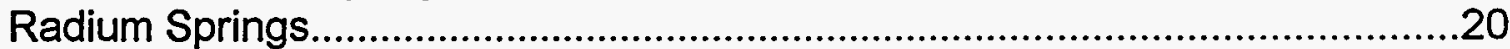

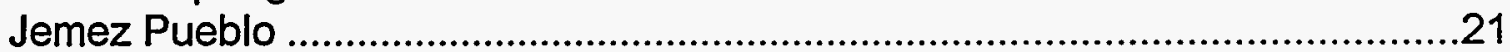

Jicarilla Apache Reservation.......................................................................22

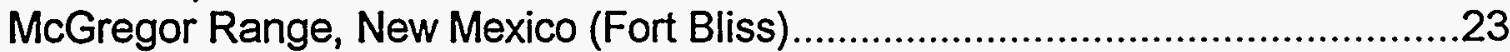

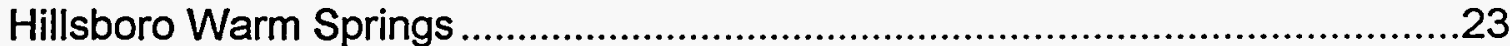

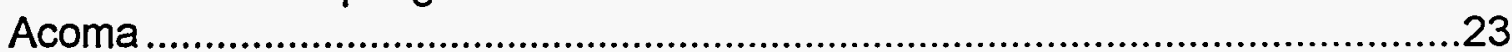

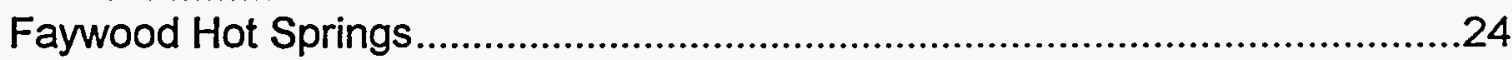

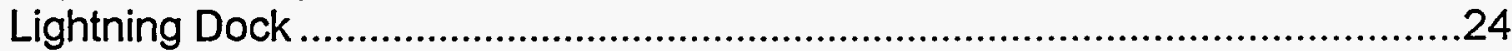

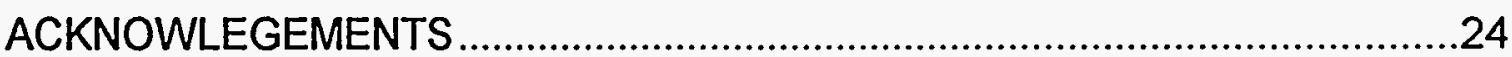

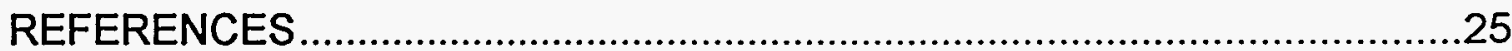




\section{APPENDICES}

APPENDIX 1 Geothermal sites and location data tables

APPENDIX 2 Tables of complete chemical analyses

APPENDIX 3 Tables of partial chemical analyses

APPENDIX 4 Site and sample information tables

APPENDIX 5 References for data sources

RECFI: D

FER 23 i $=. j$

0511

APPENDIX 6 New Mexico well and spring location system 


\section{FIGURES}

FIGURE 1 Histogram of well and spring discharge temperatures.....................6

FIGURE 2 Generalized map of thermal (>30 C) wells and springs.....................7

FIGURE 3 Physiographic provinces of New Mexico .......................................8

FIGURE 4 Map showing general locations of priority study areas...................14 


\section{INTRODUCTION}

This report provides a compilation of geothermal well and spring information in New Mexico up to 1993. Economically important geothermal direct-use development in New Mexico and the widespread use of personal computers (PC) in recent years attest to the need for an easily used and accessible data base of geothermal data in a digital format suitable for the PC. This report and data base are a part of a larger congressionally-funded national effort to encourage and assist geothermal direct-use. In 1991, the U. S Department of Energy, Geothermal Division (DOE/GD) began a LowTemperature Geothermal Resources and Technology Transfer Program. Phase 1 of this program includes updating the inventory of wells and springs of ten western states and placing these data into a digital format that is universally accessible to the PC. The Oregon Institute of Technology GeoHeat Center (OIT) administers the program and the University of Utah Earth Sciences and Resources Institute (ESRI) provides technical direction.

Since 1980, New Mexico has had significant direct-use geothermal development. In 1982, one of the nation's larger district heating systems began operation at New Mexico State University. In 1986, a geothermally-heated geothermal greenhouse research and business 'incubator' facility came on line through a combination of private donations and State funds and is operated by the Southwest Technology Development Institute (SWTDI/NMSU), a division of the Engineering College at New Mexico State University (Schoenmackers, 1988). The first client in the NMSU greenhouse now operates the nation's second largest geothermally-heated commercial greenhouse at Radium Springs, New Mexico. Currently, New Mexico has the largest acreage of geothermal greenhouses in the nation with more than 40 acres $\left(161,900 \mathrm{~m}^{2}\right)$. This acreage is about half of the total greenhouse acreage in New Mexico and represents an estimated capital investment of more than $\$ 30$ million and the direct creation of nearly 400 jobs.

So far in the 1990's, interest and growth has continued in using geothermal heat in New Mexico. Primary interest is from the agriculture sector, including greenhousing, aquaculture, crop and food processing, and milk and cheese processing. Other interest has included space heating and heated swimming pools. This data base will assist in further direct-use geothermal efforts. 


\section{PREVIOUS COMPILATIONS}

The first statewide evaluation and compilation of geothermal information for New Mexico was begun in the mid 1960's and resulted in New Mexico Bureau of Mines and Mineral Resources Hydrologic Report 4, 'Catalog of Thermal Waters in New Mexico' (Summers, 1976). Summers (1976) remains the primary source information on New Mexico thermal springs. During the mid 1970's and early 1980's, Federal and State geothermal resource characterization efforts led to additional information collection efforts. Two U. S. Geological Survey (USGS) Circulars provided the initial estimates of resource size and quality (Muffler, 1979; and Reed, 1983). In addition, a cooperative effort between the U.S. Department of Energy (DOE), the National Oceanic and Atmospheric Administration (NOAA), and New Mexico State University resulted in 1:500,000 scale geothermal resource maps (Swanberg, 1980 and Swanberg and Icerman, 1983). Prior to 1983, geothermal data for New Mexico were included in GEOTHERM, a USGS mainframe computer system of geothermal data bases and geothermal evaluation software (Bliss and Rapport, 1983). The USGS discontinued GEOTHERM in 1983. More recently, a relational database system for the PC platform was developed at NMSU for geothermal information covering New Mexico (Witcher and others, 1990). Limited data compilation and new and easier to use relational database software make the 1990 database obsolete.

\section{DATA SOURCES}

Major statewide sources of data include Summers (1976), Swanberg (1980), Norman and Bernhardt (1982). A major source of statewide information is contained in the U. S. Geological Survey (USGS) WATSTORE file. WATSTORE has two major databases, the Ground-Water Site Inventory and the Water Quality File. A 1993 commercial version of the WATSTORE Water Quality File on CD ROM was used in this study.

Additional important data for the geothermally significant Jemez Mountains (Valles Caldera) region in north central New Mexico is found in Shevenell and others (1987).

The state geothermal resources maps and the USGS GEOTHERM file were reviewed for data and used to assist in the compilation. However, neither 
the maps or the GEOTHERM file are primary information sources for the type of data compiled in this study.

Additional information was compiled from published and unpublished site specific geothermal resource investigations at several locations. Other data was compiled from published ground-water studies and government open-file reports. Finally, it should be noted that this study is not an exhaustive compilation of data for geothermal wells and springs in New Mexico. Except for a few sites at high elevations, the only data compiled was for wells and springs with measured discharge temperatures greater than $30^{\circ} \mathrm{C}$. Virtually all wells and springs found at elevations below 5,000 feet $(1,524 \mathrm{~m})$ elevation in New Mexico exceed $20^{\circ} \mathrm{C}$.

In addition, sites based upon bottom-hole temperature data are not included in this data base. The 1980 state geothermal map includes bottomhole temperature data. Also, no heat-flow or temperature-gradient data is included in this compilation. These data sets require analysis and interpretation beyond the scope of this project. The Southwest Technology Development Institute at NMSU has extensive compilations of heat-flow and bottomtemperature data for New Mexico.

\section{DATA FORMAT}

Three Excel@ (Microsoft Windows@ software) spreadsheets provided a working medium for data compilation, editing, and sorting. The first spreadsheet (Appendix 1) lists the geothermal sites and provides location information. Location data in many cases is poor quality and may be only accurate to a minute of latitude or longitude. Field experience shows that this is true of some WATSTORE data as well data from other sources. Field checks and determination of UTM coordinates are required to improve the locations at most sites.

The second spreadsheet lists 'complete' chemical analyses for geothermal sites in New Mexico (Appendix 2). Data in the second spreadsheet contains at a minimum a dissolved silica analysis and sufficient major cation ( $\mathrm{Na}$, $\mathrm{K}, \mathrm{Ca}, \mathrm{Mg}$ ) and major anion ( $\mathrm{Cl}, \mathrm{HCO}, \mathrm{SO} 4)$ data to check for analytical charge and mass balance (see Reed and Mariner, 1991). Each analysis for geothermal sites in New Mexico is assigned a unique sample identification if the original data source failed to provide this information. This approach assists in duplicate record checking and provides a foundation to include these data in a relational 
data base and Geographic Information System (GIS) for New Mexico geothermal information in the future.

The third spreadsheet lists 'partial' chemical analyses (Appendix 3). These data do not satisfy the criteria for the second spreadsheet. Also, the third spreadsheet has an added entry that shows sodium and potassium as a single analysis $(\mathrm{Na}+\mathrm{K})$ as is commonly reported in older citations. In general, the third spreadsheet may have lower quality data than those found in the second spreadsheet ('complete analysis'). Caution is advised in applying chemical geothermometers or in assessing potential for corrosion and scaling with the data in the third spreadsheet ('partial analysis'). The same caution applies to using data in the second spreadsheet with significant charge and mass balance errors (greater than 5 or 10 percent).

Except for the GEOTHERM and WATSTORE information, data was manually (keyboard) entered. WATSTORE data was extracted from the CD ROM data base by sequentially retrieving all analytical data for individual sites with measured temperatures greater than $30^{\circ} \mathrm{C}$ and placing these data in an ASCII master file using software provided by the data vendor. A small FORTRAN program was written and used to read the ASCII master file and retrieve specific analyses and to organize these data into a tabular ASCII file that can be opened by Excel@ and placed directly into the formatted spreadsheets.

\section{OVERVIEW OF THE DATA BASE}

The last comprehensive geothermal data compilation in 1980 (state geothermal map - Swanberg, 1980) displayed 312 thermal wells and springs. Many sources shown on the 1980 map are bottom hole temperature (BHT) measured either during geophysical logging of oil and gas exploration wells or from academic heat flow studies. No BHT data are included in this compilation. GEOTHERM lists 65 chemical analysis of New Mexico thermal wells and springs (Reed and others, 1983).

This data base contains 842 chemical analysis for 360 discrete thermal wells and spring discharges. About half of the sites (175 sites) are extracted from WATSTORE. The remaining data are taken from published and unpublished reports. 
Figure 1 is a histogram that shows the relative frequency of measured surface discharge temperatures for 308 well and spring sites. Data for high temperature $\left(>150{ }^{\circ} \mathrm{C}\right.$ ) test wells in the Jemez Mountains (Valles or Baca geothermal system) are excluded from the histogram. A median temperature of about $35^{\circ} \mathrm{C}$ is evident. On a percentile basis, measured temperatures above 46 ${ }^{\circ} \mathrm{C}$ score 75 or higher while temperatures above $62{ }^{\circ} \mathrm{C}$ score 90 and above. With hot spring data removed, the remaining data for the greater than $62^{\circ} \mathrm{C}$ category are from wells in three developing geothermal areas, Lightning Dock, Radium Springs, the Las Cruces East Mesa. Many, if not most, data in the 30 to $40{ }^{\circ} \mathrm{C}$ bracket are from deep wells with conductive thermal regimes (normal or slightly above regional temperature gradient averages). It is clear that a developer will need to drill new wells in areas with convective geothermal resources in order to obtain resource temperatures over $45^{\circ} \mathrm{C}$. On the other hand, if temperatures below $45^{\circ} \mathrm{C}$ are required, there are many existing sites to evaluate.

Figure 2 is a map of New Mexico which summarizes the locations of thermal (mostly $>30^{\circ} \mathrm{C}$ ) wells and springs. Several areas are notable when Figure 2 is compared to the 1980 and 1983 compilations (Swanberg, 1980 and Swanberg and Icerman, 1983). A new region with low-temperature potential is indicated in the Pecos Valley in southeastern New Mexico in Chaves and Eddy County. Numerous wells between 26 and $29^{\circ} \mathrm{C}$ occur in the area of Eddy and Chaves Counties. Two wells, $30{ }^{\circ} \mathrm{C}$ or warmer, are shown in Figure 2. Aquaculture is one possible use for the low-grade thermal waters. Recent analysis of oil-and-gas well, temperature data and thermal conductivity measurement of subsurface units across the region by Reiter and Jordan (1995) suggest broad, upward cross-formational flow from depths of 3,000 to 5,000 feet (914 to $1,524 \mathrm{~m}$ ) beneath the Pecos Valley.

An extensive north-south alignment of saline, travertine-depositing springs in remote western Valencia County are not included in this compilation. However, the springs are shown on the 1980 and 1983 compilations as the Laguna springs and seeps. All of the springs discharge less than $30^{\circ} \mathrm{C}$ temperature fluids. Goff and others (1983) discuss these springs in some detail and use fluid chemistry to identify spring origins and hydrogeology.

Also, another new region is compiled in central Cibola County on the Acoma Pueblo lands. Kues (1989) briefly discusses many of the Acoma thermal wells. 


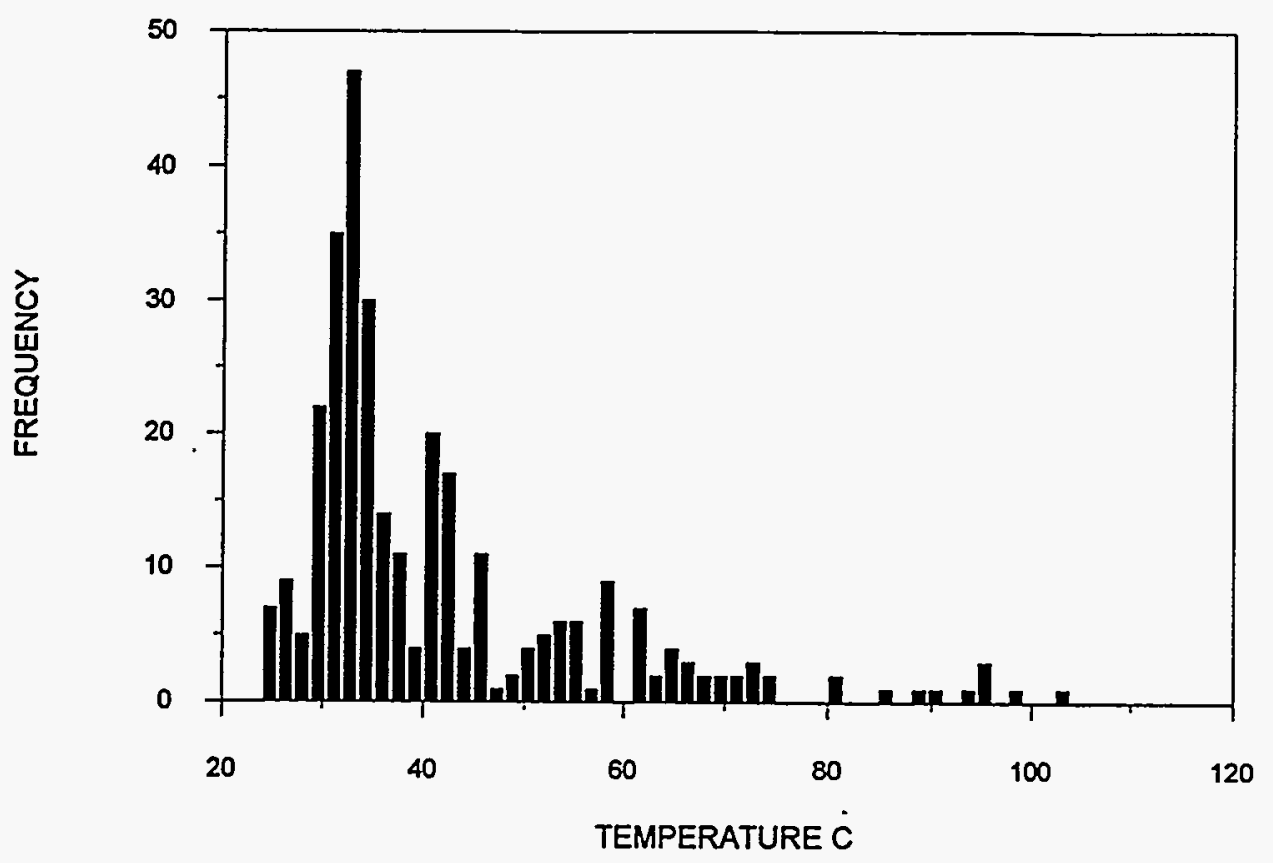

Figure 1 Histogram of well and spring discharge temperatures. 


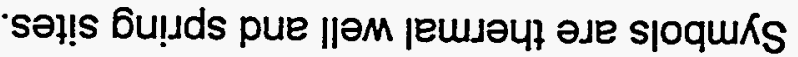

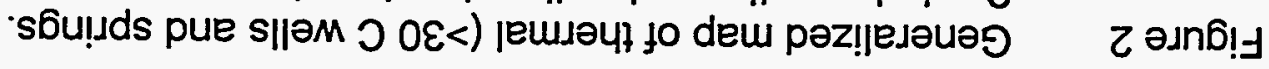

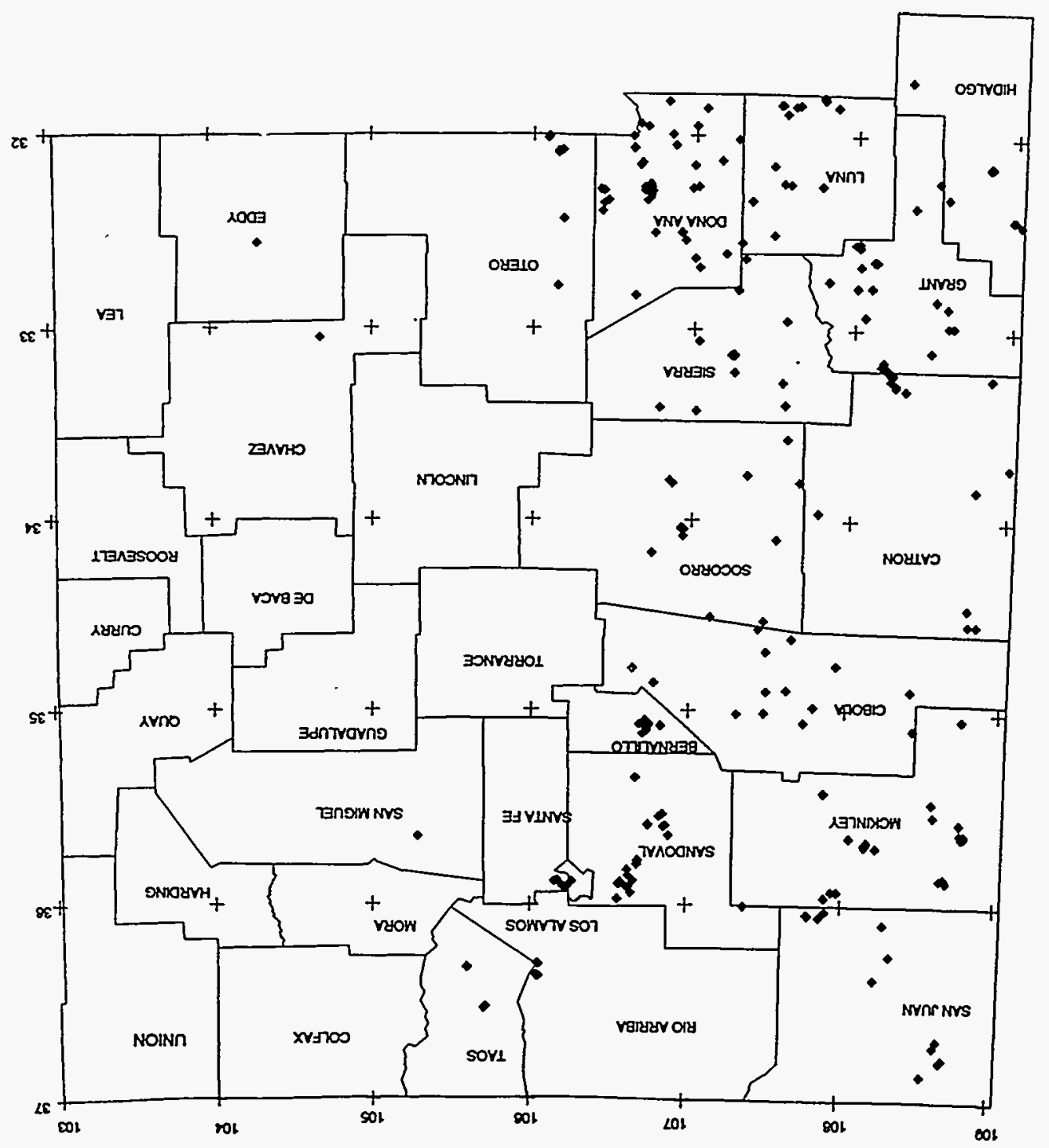




\section{DISCUSSION OF THE RESOURCE BASE}

The geothermal potential varies considerably from one area of New Mexico to the next. Regionally, the variation in subsurface temperatures is largely the result of physiographic or tectonic diversity. Physiographic provinces generally have unique geologic histories, structures, topography, hydrology, climate, and rocks. New Mexico includes four major physiographic provinces (Fig. 3). Provinces include the Southern Basin and Range (SBRP), Colorado Plateau (CP), Southern Rocky Mountains (SRMP), and the Great Plains (GPP). Three subdivisions form the Basin and Range: 1) the Sacramento section; 2) the Mexican Highland section; 3) the Datil-Mogollon section. The eastern and northern portions of the Mexican Highland section of the SBRP and the SRMP are frequently referred to as the Rio Grande rift (RGR) 'tectonic province.'

High-to-moderate heat flow $\left(>80 \mathrm{mWm}^{-2}\right)$, widely-scattered hot springs and thermal wells, Quaternary volcanism (mostly basalt), recurrent Pleistoceneto-Recent and predominantly-normal faulting indicates, by rank of overall enhanced crustal heat, that the SBRP, SRMP, and CP have elevated subsurface temperatures and significant geothermal resource potential (Swanberg, 1980; Swanberg, 1983; Summers, 1976; Morgan and others, 1986; Reiter and others, 1975, 1978, and 1986; Decker and Smithson, 1975; Reiter and Barrol, 1990; Reiter and Minier, 1989). Crustal thinning in the SBRP and Rio Grande rift has resulted in crustal thicknesses as thin as $26 \mathrm{~km}$ (Sinno and others, 1986).

\section{Cenozoic Geology}

Cenozoic geology in the geothermally-important, western two-thirds of New Mexico (SBRP, SRMP, RGR, and CP) is dominated by three major tectonic episodes: 1) the Laramide orogeny; 2) a mid-Tertiary extensional and volcanic event; 3) a late Tertiary episode of rifting.

Laramide (Late Cretaceous to Eocene) deformation includes several large north- and west-northwest- trending, basement involved uplifted terranes that exhibit one to five kilometers or more of structural relief. These 'basementcored' uplifts are frequently large-scale asymmetric homoclines with high-angle reverse faults and drape folds (monoclines) on vergent boundaries. Significant strike-slip movement occurred in other areas during the Laramide and resulted 


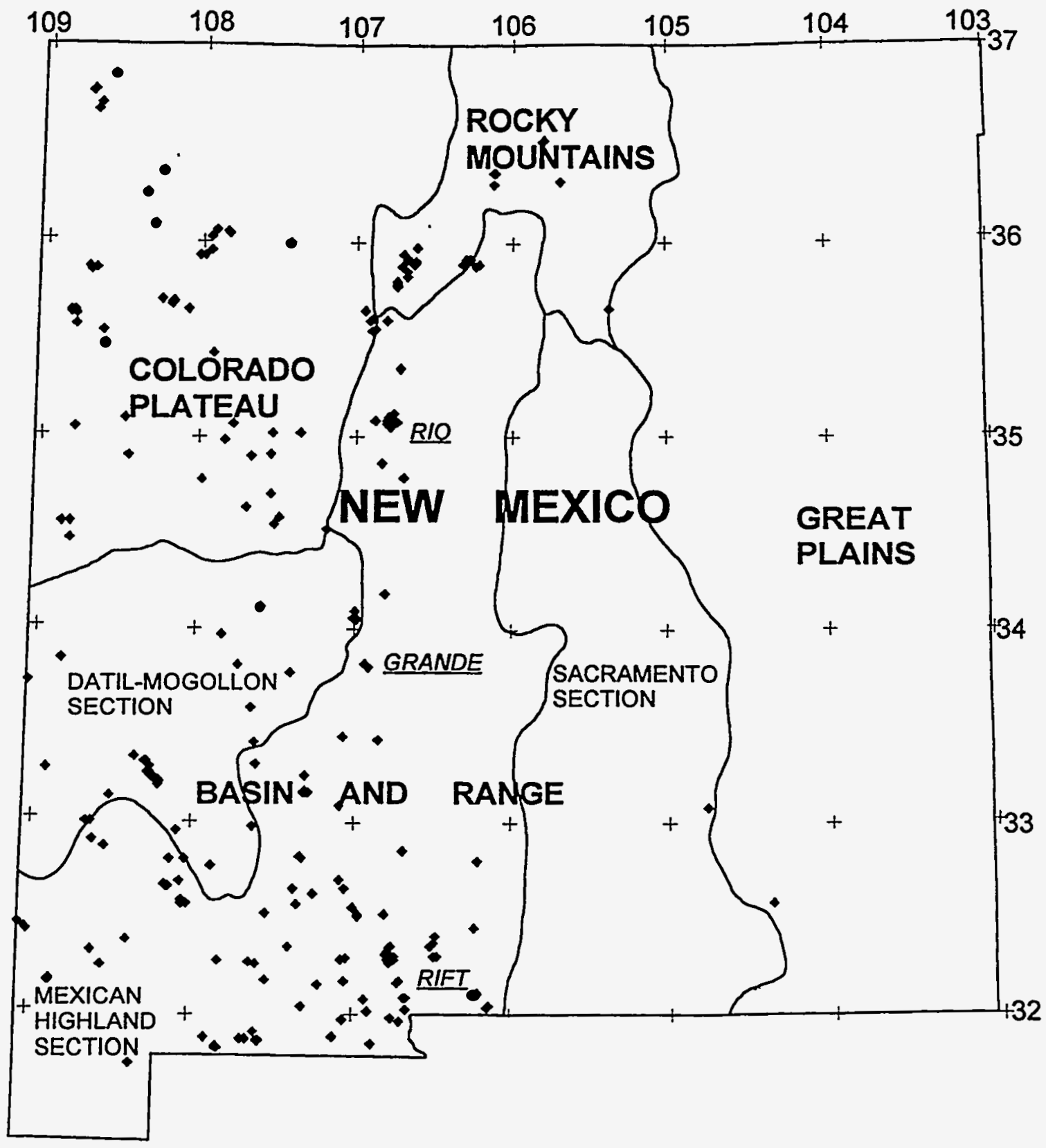

Figure 3 Physiographic provinces of New Mexico.

Symbols are thermal well and spring sites. 
in large symmetric and asymmetric transpressional structures (flower structures) which also involved basement rocks. Tertiary subcrops over these areas consist of Precambrian crystalline rocks and Paleozoic carbonate rocks. Important finegrained Mesozoic aquitards are stripped away. Virtually all convective geothermal systems in New Mexico, including the Jemez systems, occur over Laramide structural highs (Witcher, 1987 and 1988).

During the mid-Tertiary much of the Datil-Mogollon and Mexican Highland sections of the SBRP were covered by a blanket of predominantly volcaniclastic sediments and minor volcanic flows averaging one kilometer thickness (Cather and others, 1994). Flows locally dominate near volcanic centers. Regionally extensive volcaniclastic blankets provide important aquitards in the region. Locally, volcanic piles several kilometers thick occur, especially in association with silicic cauldron complexes. Many of the cauldron complexes were also the locus of intense extension (up to 100 percent) along systems of close-spaced, domino-style normal faults (Chamberlin and Osburn, 1986).

Large, widely-spaced normal faults largely blocked out present-day topography from 12 to 9 Ma over the SBRP and RGR in New Mexico up until 4 to $6 \mathrm{Ma}$ (Seager and others, 1984). This late Tertiary rifting continues at lower rates today and has left an en echelon series of north-trending half grabens with extension amounting to no more than 10 or 15 percent. Many of the best geothermal systems in New Mexico occur where late Tertiary horsts intersect older highly-extended cauldron complexes and vergent boundaries of Laramide uplifts (Witcher, 1988). Late Tertiary horsts are frequently stripped of midTertiary volcaniclastic aquitards to expose underlying fractured terranes.

Convective Resources

\section{Occurrence Models}

Several models for convective geothermal resource occurrence have been proposed the Rio Grande rift and SBRP. Chapin and others (1978), Elston (1981), Elston and others (1983) show that several systems occur at the intersection highly-faulted ring-fracture zones of mid-Tertiary cauldrons, regional lineaments, and Pleistocene faults. Elston and others (1983), Jiracek and Smith (1976), and Swanberg (1975) observe that late Tertiary fault zones apparently control other geothermal systems. 
A model of forced convection through Tertiary basin-fill sediments was presented by Harder others (1980) and Morgan and others (1981). This model places geothermal discharges at surface hydrologic outlets and down-gradient structural boundaries of late Tertiary rift basins. This model is commonly referred as the 'constriction model.' Many systems in the Rio Grande rift appear to occur at basin 'constrictions' and the model is commonly cited in the literature to explain the Rio Grande rift geothermal resource base and associated thermal regimes. Actually, the model poorly predicts discharges on a local scale and fails to explain the predominance of system upflow zones in fractured bedrock. In fact, vertical flow across major regional aquitards, followed by horizontal flow across major fault zones, which usually act as flow-regime boundaries, is required to explain many geothermal system locations relative to a 'constriction model.'

Another model which allows forced, free, or a combination of convective processes is proposed by Witcher (1988). With this model, convective geothermal systems occur in fractured bedrock (structurally-high terrane) at low elevation within horst blocks. Fluid circulation depths are not restricted by graben structural relief and the systems are not confined to areas adjacent to horst-bounding faults, as predicted by a constriction model. A regional view of New Mexico convective occurrences indicates that virtually all known systems occur where aquitards or confining units have been stripped by faulting or by erosion from basement terranes which contain significant vertical fracture permeability. A variety of structures, ranging from faults, folds, and fractured stocks and dikes can provide local vertical permeability and reservoirs. This model is referred to as a 'hydrogeologic window model.'

\section{Conductive Resources}

\section{Basin and Range and Rio Grande Rift}

Half grabens, forming the southern Basin and Range and Rio Grande rift, may contain several thousand of feet of Cenozoic sediments in various stages of diagenesis, compositional and grain-size ranges, and degrees of structural deformation. Because of the region's high heat flow and general tendency of Cenozoic basin fills to have significant fine-grained lithologies with low thermal conductivity and low vertical permeability, deep-seated and permeable 
sediments, especially fractured and faulted older basin fill units, provide potential for large-volume conductive geothermal resources. In general, the cost of deep wells is a drawback to the use of the resource. However, existing deep water supply wells and irrigation wells have potential for use.

\section{Colorado Plateau}

The eastern Colorado Plateau, including the San Juan Basin and the Mogollon Slope, has generally high flow (Minier and Reiter, 1991, and Reiter and Mansure, 1983). Locally, heat flow can be as high as that observed in the Rio Grande rift and southern Basin and Range. Significant thicknesses of finegrained Cenozoic and Mesozoic sediments are preserved over permeable lower Mesozoic redbed sands and Paleozoic redbed and carbonate aquifers. Because the bulk of the Cenozoic and Mesozoic fine-grained sequences act as aquitards and have low thermal conductivity, they act as thermal blankets to create a deep-seated conductive geothermal resource. Much of this conductive resource is under sufficient artesian pressure to allow flowing wells to be drilled and developed. A drawback to this resource, however, is fluids with high salinity, few geological alternatives for fluid injection, and the general remoteness of this region of New Mexico. Much of this region is covered by the Navajo, Zuni, Acoma, Laguna, and Jicarilla Reservations.

\section{PRIORITY AREAS AND AREAS WITH NEAR-TERM UTILIZATION POTENTIAL}

Several areas in New Mexico are identified as priority sites for near-term, low-to-intermediate temperature geothermal resource utilization. Identified areas should receive additional site specific geologic and feasibility studies. Selection is based upon several criteria. Potential quality of the resource is important. The resource quality is an engineering economics and feasibility problem as much as it is a geologic problem. Higher temperatures and highly productive shallow wells are most favorable. However, many other factors are required for development success. Resource co-location with users and other geographic attributes specific to particular direct-use applications are crucial.

Space heating and district heating are most feasible in areas where the resource is co-located with population and facilities with large heating loads. Geothermal heating has potential to be incorporated without retrofit of existing 
heating systems in some areas of New Mexico that are experiencing rapid growth.

Geothermal greenhouse heating requires a favorable land status to include costs and ownership, availability of nearby fresh water, a labor force, good transportation infrastructure or nearness to markets. Almost all of New Mexico has the sunshine and climate that growers need. Availability and cost of water rights may be an issue in some areas because New Mexico is an arid region.

While aquaculture is less labor intensive than greenhousing, a favorable land status and transportation or nearness to markets is necessary. Availability and cost of water rights may be an issue in some areas.

New Mexico has a rapidly growing dairy industry. Milk and cheese processing are very energy intensive. A high quality geothermal resource that is easily accessible and near dairies may have much potential energy savings and economic benefits. Other users include chile, onion, and other agricultural processors. Good transportation and year around product availability are desired.

Low-to-intermediate temperature geothermal direct-use utilization has much potential to enhance or create economic opportunities. This makes geothermal energy, a relatively unknown alternative to conventional fossil fuels, much more marketable. Most of the priority areas selected in New Mexico have geothermal resources co-located in areas with many favorable geographic and demographic attributes for specific end users. Most importantly, all of the priority areas have on-going private sector, Indian tribal, and/or municipal development and exploration activities or serious interest in development. Success in these areas will no doubt spawn additional interest and economic development centered on geothermal energy in New Mexico.

Selected areas cover a broad range of representative geologic and hydrologic settings favorable for economic geothermal resources in New Mexico. Areas are located in both southern and northern New Mexico. Figure 4 shows the locations of the areas to be discussed in this report. 


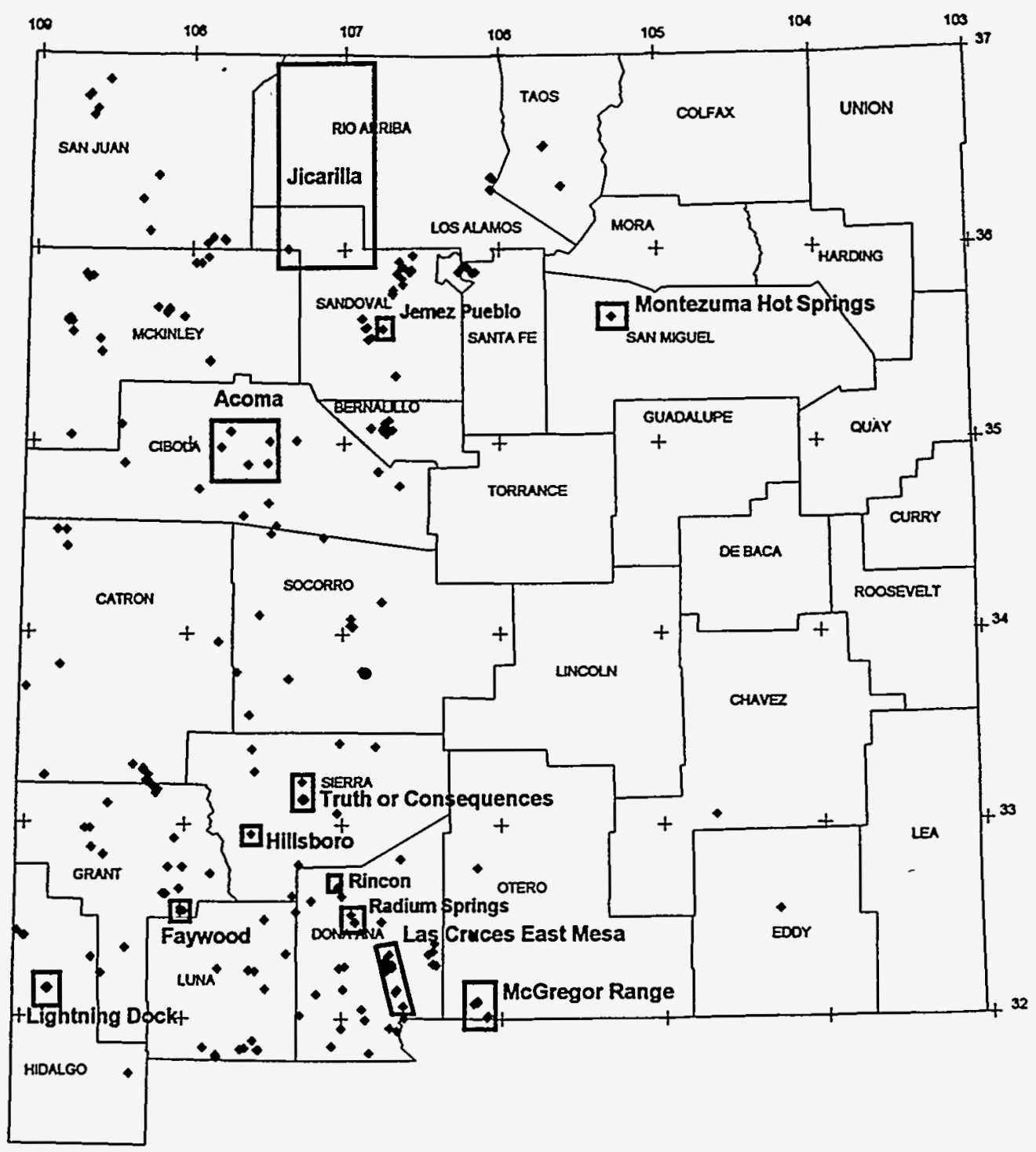

Figure 4 Map with general locations of priority study areas. Symbols are thermal well and spring sites. 
Rincon

The Rincon geothermal system is a blind system (no surface hot springs). However, Pleistocene opal beds (fossil siliceous hot spring (?) deposits) are interbedded at this site in ancestral Rio Grande fluvial deposits exposed in escarpments adjacent to the present-day downcut Rio Grande floodplain.

A recently drilled continuous-core $(H Q)$ borehole to 1,218 feet $(371 \mathrm{~m})$ at the site indicates a shallow highly-fractured reservoir from 300 to 600 feet ( 91 to $183 \mathrm{~m}$ ) depth with a temperature of 85 to $90^{\circ} \mathrm{C}$ in pervasively silicified ancestral Rio Grande fluvial deposits (Witcher, in preparation). The top of the reservoir is roughly the same level as the present day water table in the Rio Grande flood plain. The silicified zone is accompanied by adularia and disseminated sulfide mineralization. This zone is a part of the upflow zone for a much hotter and deeper-seated reservoir located in a fault zone dipping east beneath the core hole total depth. Between 600 and 1,218 feet (183 to $371 \mathrm{~m}$ ), the core hole encountered a relatively unaltered clayey siltstone which acts as an aquitard or aquaclude. Temperature gradients are $250{ }^{\circ} \mathrm{C} / \mathrm{km}$ in the lower 200 feet $(61 \mathrm{~m})$ of the hole. The bottom hole temperature is $100^{\circ} \mathrm{C}$. Geothermometer estimates indicate reservoir temperatures in the fault zone between 120 and $175^{\circ} \mathrm{C}$. The core hole was funded by the State of New Mexico Legislature.

The geothermal area is bounded on the south by Interstate 25 and on the east by an Atchison, Topeka and Santa Fe rail about 25 miles $(40 \mathrm{~km})$ north of Las Cruces in southern New Mexico. This area is an important agricultural area along the Rio Grande. The town of Hatch is located 5 miles $(8 \mathrm{~km})$ west of the site and is the locus of chile growers. The area is well known for the quality of chile produced. Large dairies are located a few miles west of Hatch. Fresh water is available within one to two kilometers of the Rincon site. Political support for geothermal development at Rincon includes the town of Hatch, the City of Las Cruces, Dona Ana County, and the State of New Mexico.

Reservoir production rates have not been determined at the site and some infrastructure work is needed to include land leveling. The land status is Federal Bureau of Land Management (BLM). Development will require a surface use license and such a license could be the first granted by the BLM for geothermal direct-use. Potential geothermal uses at this site include greenhouses, milk and cheese processing, chile processing, refrigerated warehousing, and binary electrical power. To date, exploration has included a 
slim-hole continuous core hole, 4 shallow temperature gradient holes (Witcher, 1991), a radon soil-gas survey (Witcher, 1991), a detailed SP survey (Ross and Witcher 1992).

Completion of geologic mapping at 1:6,000 scale and study of the core is needed. A shallow (600 feet or $183 \mathrm{~m}$ maximum) production hole is required to begin geothermal development at this site. Also, re-entering the core hole with an NQ drill string (the HQ string is currently in the hole) will determine deeper production and temperatures. Preliminary feasibility studies for direct use application have been performed. Detailed feasibility, production drilling, and infrastructure work is required for geothermal utilization at Rincon.

The Rincon resource has very high priority because it provides a case study for new exploration strategies and geologic occurrence models for 'blind' resources in the Rio Grande rift and southern Basin and Range capable of producing intermediate-temperature fluids for higher-end direct use and binary power production.

\section{Truth or Consequences ( $\mathrm{T}$ or $\mathrm{C}$ )}

The town of Truth or Consequences ( $T$ or $C$ ) was formerly called Hot Springs before being renamed after a 1950's television game show as a part of promotional effort. $T$ or $C$ is a retirement and resort town of about 5,000 located along the Rio Grande near Elephant Butte Reservoir, one of the largest manmade lakes in the Southwest. Numerous shallow hot artesian wells exist in the downtown area of $\mathrm{T}$ or $\mathrm{C}$. These wells have been used for most of this century in health spas. Generally, temperatures range from 40 to $43.3^{\circ} \mathrm{C}$. The aggregate flow from this system is estimated at 1,314 acre/ft per year $\left(1.6 \times 10^{6}\right.$ $\mathrm{m}^{3}$ ). All of the wells have high-priority water rights and are a part of one of the first State Engineer declared ground-water basins in New Mexico.

Small-scale space heating is done at the Geronimo Springs Museum and the Carre Tinglely veterans center and in the spas around town. In recent years, a citizens group has been interested in using geothermal heat in a heated municipal swimming pool. One of the drawbacks to geothermal development at $T$ or $C$ has centered on the reluctance of spa owners to support additional geothermal use in the downtown area.

A variety of evidence suggests that a larger and hotter geothermal system may exist outside of town and away from the Rio Grande in the vicinity of the 
Mud Springs Mountains. The currently known geothermal area probably represents outflow that has mixed with cold shallow ground water that is associated with the Rio Grande. Beds of opal (fossil hot spring (?) deposits) occur near the top of Pleistocene ancestral Rio Grande fluvial deposits. One bed is exposed in a road cut along $1-25$. Some manganese mineralization also occurs in nearby fluvial sand deposits. Late Pleistocene faults occur near the mineralized area. However, the most important structures are Laramide (Late Cretaceous to early Eocene) reverse faults that extend from $T$ or $C$ westward along the southwest margin of the Mud Springs Mountains. Such structures appear to provide first-order structure and deep plumbing for geothermal systems at Rincon, Radium Springs, Derry Warm Springs, and San Diego Mountain further south along the Rio Grande.

Some exploration has been done in the area including electrical resistivity (Jiracek and Mahoney (1981), heat flow studies (Sanford and others, 1979), reconnaissance geologic mapping in the Mud Springs Mountains and hydrogeologic evaluation (Wells and Granzow, 1981). Reported heat-flow measurements are insufficient to shed much information on the system as they are located far from the noted mineralization and probable structural control. The resistivity surveys map areas of low resistivity and steep resistivity gradients in the area of interest.

A hotter geothermal resource located north and west of $T$ or $C$ has potential for space heating, district heating, geothermal greenhousing and aquaculture. A 'phase 1' exploration effort is required to identify and confirm a probable system outside of downtown $\mathrm{T}$ or $\mathrm{C}$. This effort should be concentrated near mineralized Pleistocene sediments of the ancestral Rio Grande. Detailed mapping (1:12,000 scale) of Quaternary geology and bedrock geology at the southeastern end of the Mud Springs Mountains should be performed. A thorough hydrochemical study of the known system in downtown $\mathrm{T}$ or $\mathrm{C}$ would be useful to evaluate mixing and probable sources of the water. Radon soil-gas, soil mercury, and SP surveys may identify potential upflow zones for shallow temperature gradient evaluation. If a system is found, sufficient local support would likely be generated to pursue use in the near future. In any case, more will be learned on the nature of the known resource in $T$ or $C$ and how to manage the resource so that current users interests are better insured in the future. 


\section{Las Cruces East Mesa/Tortugas Mountain}

One of the largest 'convective' low-temperature geothermal systems in the United States occurs east of Las Cruces and southward along I-10 and I-25 nearly to the Texas line. This system, as outlined by more than 70 shallow temperature-gradient holes, is nearly 20 miles $(32 \mathrm{~km})$ long and 2 to 3 miles ( 3.2 to $4.8 \mathrm{~km}$ ) wide over a buried horst block (Lohse and Icerman, 1982). Reservoir temperatures range from 40 to $70{ }^{\circ} \mathrm{C}$. Production wells at New Mexico State University, and various industry exploration wells indicates a highly productive fractured reservoir system in Paleozoic carbonate rocks, Tertiary volcanic rocks, and older Tertiary basin-fill sediments. Production in excess of $1,000 \mathrm{gpm}$ (63 $\mathrm{L} / \mathrm{s}$ ) has been demonstrated by NMSU production well PG-4 and inferred by the Chaffee geothermal test wells (Cunniff and Chintawongvanich, 1985). Available heat-flow data indicates that the total heat loss of the system exceeds $50 \mathrm{MWt}$ with a natural mass flux exceeding 15,000 acre/ft per year $\left(18 \times 10^{6} \mathrm{~m}^{3}\right)$ of $70{ }^{\circ} \mathrm{C}$ water (Witcher and Schoenmackers, 1990).

Current use of geothermal includes a district heating system for the New Mexico State University campus, a research and business start-up (incubator) greenhouse and aquaculture facility operated by SWDTI at NMSU, and a commercial 2 acre $\left(8,093 \mathrm{~m}^{2}\right)$ greenhouse operated by $\mathrm{J} \& \mathrm{~K}$ Growers. J \& K Growers leased the SWTDI/NMSU facility to startup their business prior to moving to their present location. J \& K Growers have increased the size of their greenhouse business annually.

Near-term geothermal utilization includes more geothermally-heated commercial greenhouses, aquaculture, and space heating of large buildings to include schools, hotels, and businesses. District residential heating is believed to be only marginally feasible due to generally mild winters. Access to the area is good and much of the resource is adjacent to the city or within the city limits.

The U. S. Bureau of Land Management has recently designated more than $40 \mathrm{~km}^{2}$ of the area as a KGRA. This action discourages direct-use operators by adding additional time, paper work, and risk to business planning and execution.

A major attribute of this resource is co-location with one of the fastest growing medium-sized cities in the United States. In fact, the city of Las Cruces is growing in the direction of the resource at a rapid rate. Definitive integration of geothermal energy into city and public school planning is needed. Some 
feasibility studies were conducted in cooperation with the city and SWTDI/NMSU more than 10 years ago for existing large buildings near, but outside, the resource area (Icerman and Whittier, 1983; and $\left.\mathrm{CH}^{2} \mathrm{MHILL}, 1984\right)$. These studies need to be updated and applied to plans and projections of growth over the resource area proper. A significant cost-shared drilling or a demonstration project in the commercial or local government sectors and outside of the NMSU area is probably needed to fully realize the potential for Las Cruces. City and county officials are aware of geothermal energy and have been supportive of SWTDI/NMSU initiatives at Rincon and Radium Springs. However, momentum and general awareness needs to be fostered for potential of the Las Cruces East Mesa geothermal resource.

Montezuma Hot Springs/United World College

The Armand Hammer United World College at Montezuma Hot Springs near Las Vegas, New Mexico is interested in using geothermal energy for space heating the college in order to replace a coal-fired boiler. Sandia National Laboratory, Los Alamos National Laboratory and SWTDI/NMSU have teamed up to provide UWC with geotechnical and engineering services.

Initial 'phase 1' work is needed to determine the production potential of the resource and to determine the influence that a production well will have on the long-term flow of Montezuma Hot Springs. Resource quality and degradation potential on natural spring flow rate will dictate the geothermal heating approach. The least expensive alternatives are direct-use space heating or the use of ground water-coupled heat pumps.

The gross structural control for the Montezuma Hot Springs is a southern Rocky Mountain 'front range' structure consisting of a Laramide reverse fault and attendant fold structure that forms the vergent margin the basement-cored Sangre De Cristo uplift (Baltz, 1972; and Bejnar and Bejnar, 1979). This structure and others of similar nature continue northward into Colorado. It is possible that additional geothermal systems occur elsewhere on this trend, especially in the Mora, New Mexico area north of Las Vegas. It is also possible that Montezuma Hot Springs proper represents a larger local geothermal system that could provide geothermal for more users than UWC.

SWTDI/NMSU was recently awarded a contract from the State of New Mexico Department of Economic Development that will be used to provide a 
state match for a 'phase 1' evaluation of the resource at Montezuma Hot Springs. Sandia and Los Alamos labs have authorization from the U. S. DOE to apply federal funds as a match in the labs joint efforts in the 'phase 1' work.

A successful geothermal heating system at UWC will provide a high visibility demonstration of geothermal technology in northern New Mexico. Also, if the resource base in the area is determined to be larger, significant economic benefits will accrue to this economically depressed region of New Mexico.

\section{Radium Springs}

Radium Springs is the site of the second largest geothermally-heated greenhouse in the United States at 9.5 acres $\left(38,440 \mathrm{~m}^{2}\right)$. At the present time, construction is proceeding on two additional acres $\left(8,000 \mathrm{~m}^{2}\right)$ and land is being prepared for 4 more acres $\left(16,000 \mathrm{~m}^{2}\right)$ of greenhouse. Prior to building the facility at Radium Springs, Alexander Masson of Lindeville, Kansas leased space in the SWTDI/NMSU greenhouse facility at Las Cruces in order to assist with business startup in New Mexico.

While the Masson greenhouse at Radium Springs is on private land, most of the area is either a part of the Radium Springs KGRA or the NMSU Research Ranch. The extreme southern and northern areas are located adjacent to fresh water that occurs in Rio Grande flood plain alluvial deposits.

The geothermal resource in the area is extensive across an area 3 miles $(4.8 \mathrm{~km})$ wide by 10 miles $(16 \mathrm{~km})$ in length, extending from Radium Springs on the south to San Diego Mountain on the north (Witcher and Schoenmackers, 1990). Deep drilling $(8,000$ to 9,000 feet or 2,438 to $2,743 \mathrm{~m}$ ) by Hunt Energy indicates a deep reservoir in Paleozoic carbonate and Precambrian granite. Temperatures in the deep reservoir are not known, but they are probably between 100 and $150^{\circ} \mathrm{C}$. Several shallow and isolated reservoirs or upflow zones in fractured rhyolite dikes and plugs and fault zones provide discharge to the near surface from the deep system. With wells less than 250 feet $(76 \mathrm{~m})$ deep, the Masson greenhouse taps 65 to $70^{\circ} \mathrm{C}$ water that is contained in a large rhyolite dike.

Because of the limited extent of the upflow vertical permeability, nearness to the Rio Grande, and increasing geothermal production, studies are needed to detail shallow reservoir interaction with the Rio Grande and coupling of production and injection. These are potential problems that greenhouse 
operators, with lay knowledge of geology and hydrogeology, have trouble understanding and are generally not receptive to spending money for monitoring and evaluation. This attitude is probably universal in early development experience, even among engineers, geologists, and managers, as experience in over development of ground water in the arid West demonstrates. With directuse geothermal there are few case studies that directly address these types of sustained production problems. A large geothermally-heated commercial greenhouse is a significant investment and provides much economic benefits through jobs, taxes, and local purchases. Cost-shared private and government funded studies may be a way to quantify and understand the best way to proceed with development and monitor a low-temperature resource that is palatable to pioneer operators in the current early stages of low-temperature geothermal development in the United States.

Jemez Pueblo

A poorly explored resource occurs within 1.5 miles $(2.4 \mathrm{~km})$ of the Jemez Pueblo in northcentral New Mexico. This resource represents a distal discharge from the outflow plume of the high-temperature Baca geothermal system in the Jemez Mountains (Witcher and others, 1992). Reconnaissance exploration by SWTDI/NMSU includes geologic mapping, geochemistry, a detailed gravity profile, and one mile $(1.6 \mathrm{~km})$ of shallow seismic reflection survey in two profiles, and a State Legislature funded shallow exploratory well (Witcher, 1988, 1990, and 1991). This well produces a $250 \mathrm{gpm}(15.8 \mathrm{~L} / \mathrm{s})$ artesian flow of $57.8^{\circ} \mathrm{C}$ water with $3,366 \mathrm{mg} / \mathrm{L}$ TDS. Fresh water is co-located with this shallow resource.

Tribal officials are very interested in using the geothermal resource as a spring board for much needed economic development to provide income and jobs for the Jemez People. The resource is also located near the Pueblo and it may be feasible to economically provide space heating. Potential uses include geothermal greenhousing, a spa/resort, geothermal aquaculture, and district heating.

Drawbacks include slow tribal decision making which results from frequent change over in tribal leadership. Also, resource utilization will require suitable heat exchangers and materials to control corrosion. 
SWTDI/NMSU and pueblo officials are currently discussing approaches for detailed feasibility and action/business plans for direct-use geothermal utilization as a next step toward the eventual use of the resource.

Jicarilla Apache Reservation

The Jicarilla Apache are currently working with the NMSU Agriculture College on an Agricultural Sciences Center in northern New Mexico and to economically develop tribal lands. The Jicarilla Apache and the NMSU Agriculture College have invited SWTDI/NMSU to participate in evaluation of the geothermal resource base in this region. In June 1995, the Jicarilla leadership passed a resolution to begin an assessment of the geothermal resources in the region.

The Jicarilla Apache have significant oil and gas production on the southern portion of the reservation. This area is in the eastern portion of the San Juan Basin which has abnormally high heat flow for the Colorado Plateau (Minier and Reiter, 1991, and Reiter and Mansure, 1983). Heat flow is similar to the Rio Grande rift and southern Basin and Range provinces. Many petroleum wells have encountered hot saline water ranging from 50 to $110^{\circ} \mathrm{C}$, indicating a significant deep-seated conductive geothermal resource. Currently, the petroleum industry disposes much of this water in injection wells.

The northern portion of the Jicarilla Apache reservation is bounded on the north by Colorado. The area also is home to most of the tribe. Tribal headquarters are in Dulce which has harsh winters due to its elevation. The area is astride the Archuleta Arch, a.northwest trending structure extending northward into Colorado. Several important geothermal occurrences exist along this trend in Colorado, including Pagosa Hot Springs. Numerous Tertiary dikes in the Dulce area may provide vertical permeability for upflow of deep-seated conductive thermal waters.

Potential uses may include geothermal space heating, geothermal greenhousing, aquaculture, and oil field cleanup and disposal with geothermal artificial wetlands. 
McGregor Range, New Mexico (Ft Bliss)

An area covering more than $40 \mathrm{~km}^{2}$ with abnormally high temperature gradients occurs just north of the New Mexico and Texas boundary within the McGregor Range military reservation (Henry and Gluck, 1981; and Taylor, 1981). Ft Bliss (Army), in conjunction with SWTDI/NMSU and the University of Texas at El Paso (UTEP), is currently investigating the resource potential in this area to determine if geothermal can lower energy costs for Army facilities in the region.

\section{Hillsboro Warm Springs}

Hillsboro is a small community west of Truth or Consequences in the foothills of the Black Range. Ranching, mining, and tourism provide an economic base for the area. About 3 miles $(4.8 \mathrm{~km})$ north of the community, a group of thermal springs occur on private land. Temperature-gradient/heat-flow studies (Files, SWTDI), geothermometry (Swanberg, 1980 and 1984), and preliminary SP studies (Ross and Witcher, in progress) indicate potential for an intermediate temperature $\left(>90^{\circ} \mathrm{C}\right)$ resource at shallow depths. Potential use of a resource in this area may include minerals extraction, greenhousing, smallscale binary electrical power, and district heating. An important porphyry copper deposit occurs at Copper Flat about 2 miles $(3.2 \mathrm{~km})$ east of the thermal springs (Dunn, 1982).

Acoma

An potentially important conductive geothermal resource occurs in the San Andres limestone along Interstate 40 about 10 miles $(16 \mathrm{~km})$ east of Grants on the Acoma Reservation. Test drilling by the U. S. Geological Survey Water Resources Division (USGS-WRD) shows that flowing artesian thermal wells may be developed in this area (Kues, 1989; White and Kelley, 1980; and Baldwin and Anderholm, 1992). Because this area is also in the heart of the Grants uranium belt, potential uses of geothermal may include greenhousing and algae culture which produces crops suitable for mine reclamation and waste-water cleanup. Small-scale space and district heating is also possible in the near-term, 
considering the areas local climate and concentration of rural population along the Interstate.

Faywood Hot Springs

Faywood Hot Springs has recently been sold by Phelps Dodge, a major mining company, to a private individual, opening the way for possible smallscale commercial geothermal utilization. The area is well situated with respect to highway transportation.

\section{Lightning Dock}

The Lightning Dock geothermal system is certainly a high priority area as past performance in geothermal development will attest. This area currently has the largest geothermally-heated greenhouse complex in the nation at more than 30 acres $\left(121,000 \mathrm{~m}^{2}\right)$. Burgett Geothermal Greenhouses grow roses that are marketed throughout the Southwest and the rest of the country. From a hydrogeologic standpoint, it is unknown how current or future use will affect sustainable resource use.

\section{ACKNOWLEDGEMENTS}

Paul Lienau and Gene Culver of the Oregon Institute of Technology, GeoHeat Center are thanked for their patience, support, and suggestions. Also, Howard Ross, Earth Science Research Institute, University of Utah provided helpful comments and suggestions for the project. 


\section{REFERENCES}

Baldwin, J. A., and Anderholm, S. K., 1992, Hydrogeology and ground-water chemistry of the San Andres-Glorieta aquifer in the Acoma embayment and eastern Zuni uplift, west central New Mexico: U. S. Geological Survey WaterResources Investigations Report 91-4033, 304 p.

Baltz, E. H., 1972, Geologic map and cross sections of the Gallinas Creek area, Sangre De Cristo Mountains, San Miguel County, New Mexico: U. S. Geological Survey Miscellaneous Geologic Investigations Map I-673, 1:24,000 scale.

Barroll, M. W., Reiter, M., 1990, Analysis of the Socorro hydrothermal system: central New Mexico: Journal of Geophysical Research, v. 95, no. B13, p. 2194921963.

Bejnar, W., and Bejnar, K. C., 1979, Structural geology related to the Montezuma Hot Springs, Montezuma, New Mexico: New Mexico Geology, v. 2, no. 2, p. 21-24.

Bliss, J. D., and Rapport, A., 1983, GEOTHERM: The U. S. Geological Survey geothermal information system: Computers and Geosciences, v. 9, no.1, p. 3539.

Cather, S. M., Chamberlin, R. M., and Ratte, J. C., 1994, Tertiary stratigraphy and nomenclature for western New Mexico and eastern Arizona in Chamberlin, R. M., Kues, B. S., Cather, S. M., Barker, J. M., and Mclntosh, W. C. eds., Mogollon Slope, West-Central New Mexico and East-Central Arizona: New Mexico Geological Society 45th Annual Field Conference, p. 259-266.

$\mathrm{CH}^{2}$ MHILL, 1984, Feasibility of a geothermal heating utility: Technical Report prepared for the City of Las Cruces, New Mexico, 2 volumes.

Chamberlin, R. M, and Osburn, G. R., 1986, Tectonic framework, character, and evolution of upper crustal extensional domains in the Socorro area of the Rio Grande rift, New Mexico: Arizona Geological Society Digest 16, p. 464. 
Chapin, C. E., Chamberlin, R., M., Osburn, G. R., White, D. W., and Sanford, A. R., 1978, Exploration framework of the Socorro geothermal area: New Mexico Geological Society Special Publication 7, p. 115-129.

Cunniff, R. A., and Chintawongvanich, P. 1985, New Mexico State University geothermal exploratory well DT-3: New Mexico State University Physical Science Laboratory Technical Completion Report, $129 \mathrm{p}$.

Decker, E. R., and Smithson, S. B., 1975, Heat flow and gravity interpretation across the Rio Grande rift in southern New Mexico and West Texas: Journal of Geophysical Research, v. 80, p. 2542-2552.

Dunn, P. G., 1982, Geology of the Copper Flat porphyry copper deposit, in Titley, S. R., ed., Advances in Geology of the Porphyry Copper Deposits Southwestern North America: University of Arizona Press, Tucson, Arizona, chapter 14, p. 313-325.

Elston, W. E., 1981, Assessment of the geothermal potential of southwestern New Mexico: New Mexico Research and Development Institute Report EMD 2$67-2123,39 \mathrm{p}$.

Elston, W. E., Deal., E. G., and Logsdon, M. J., 1983, Geology and geothermal waters of Lightning Dock region, Animas Valley and Pyramid Mountains, Hidalgo County, New Mexico: New Mexico Bureau of Mines and Mineral Resources Circular 177, $44 \mathrm{p}$.

Goff, F. E., McCormick, T., Gardner, J. N., Trujillo, P. E., Counce, D., Vidale, R., Charles, R., 1983, Water chemistry of the Lucero uplift, New Mexico: Los Alamos National Laboratory Report LA-9738-OBES, 26 p.

Harder, V., Morgan, P. and Swanberg, C. A., 1980, Geothermal resources in the Rio Grande rift - origins and potential: Transactions, Geothermal Resources Council, v. 4, p. 61-64. 
Henry, C. D., and Gluck, J. K., 1981, A preliminary assessment of the geologic setting, hydrology, and geochemistry of the Hueco Tanks geothermal area, Texas and New Mexico: Texas Bureau of Economic Geology Geological Circular $81-1,48 p$.

Icerman, L., and Whittier, J., 1983, A preliminary evaluation of geothermal energy development by the City of Las Cruces: New Mexico State University Energy Institute Final Technical Report, $158 \mathrm{p}$.

Jiracek, G. R., and Mahoney, M., 1981, Electrical resistivity investigation of the geothermal potential of the Truth or Consequences area, in Icerman, L., Starkey, A., Trentman, N., eds., State-Coupled Low-Temperature Geothermal Resource Assessment Program, Fiscal Year 1980, Final Technical Report: New Mexico Energy Institute at New Mexico State University, p. 3-53 to 3-72.

Jiracek, G. R., and Smith, C., 1976, Deep resistivity investigations at two Known Geothermal Resource Areas (KGRA's) in New Mexico - Radium Springs and Lightning Dock: New Mexico Geological Society Special Publication 6, p. 71-76.

Kues, G. E., 1989, Well drilling, water-quality sampling, and aquifer testing on Acoma Pueblo lands 1 November 1988 to 8 March 1989: New Mexico Geological Society 40th Field Conference Guidebook, p. 29-30.

Lohse, R. L., and Icerman, L., 1982, Temperature gradient drilling in Las Cruces East Mesa geothermal field: Transactions, Geothermal Resources Council, v. 6, p. $141-144$.

Minier, J., and Reiter, M., 1991, Heat flow on the southern Colorado Plateau: Tectonophysics, v. 200, p. 51-66.

Morgan, P., Harder, V., Swanberg, C. A., and Daggett, P. H., 1981, A ground water convection model for Rio Grande rift geothermal resources: Transactions, Geothermal Resources Council, v. 5, p. 193-196. 
Morgan, P., Seager, W. R., and Golombek, M. P., 1986, Cenozoic thermal, mechanical and tectonic evolution of the Rio Grande rift: Journal of Geophysical Research, v. 91, p. 6263-6276.

Muffler, L. J. P., ed., 1979, Assessment of geothermal resources of the United States 1979: U. S. Geological Survey Circular 790, p. 163.

Norman, D. I., and Bernhardt, C. A., 1982, Assessment of geothermal reservoirs by analysis of gases in thermal water: New Mexico Research and Development Institute EMD 2-68-2305, $130 \mathrm{p}$.

Reed, M. J.,ed., 1983, Assessment of low-temperature geothermal resources of the United States 1982: U. S. Geological Survey Circular 892, p. 73.

Reed, M. J., Mariner, R. H., Brook, C. A., and Sorey, M. L., 1983, Selected data for low-temperature geothermal systems in the United States; reference data for U. S. Geological Survey Circular 892: U. S. Geological Survey Open-File Report 83-250.

Reed, M. J., and Mariner, R. H., 1991, Quality control of chemical and isotopic analyses of geothermal water samples: Proceedings, Sixteenth Workship on Geothermal Reservoir Engineering, Stanford University, p. 9-13.

Reiter, M., and Barroll, M. W., 1990, High heat flow in the Jornada del Muerto: 'a region of crustal thinning in the Rio Grande rift without upper crustal extension: Tectonophysics, v. 174, p. 183-195.

Reiter, M., Edwards, C. L., Hartman, H., and Weidman, C., 1975, Terrestrial heat flow along the Rio Grande rift, New Mexico and southern Colorado: Geological Society of America Bulletin, v. 86, p. 811-818.

Reiter, M., Eggleston, R. E., Broadwell, B. R., and Minier, J., 1986, Estimates of terrestrial heat flow from deep petroleum tests along the Rio Grande rift in central and southern New Mexico: Journal of Geophysical Research, v. 91, no. B6, p. 6225-6245. 
Reiter, M., and Jordan, D. J., 1995, Preliminary hydrogeothermal studies across the Pecos River Valley in southeastern New Mexico (Abstract): Proceedings, 1995 New Mexico Geological Society Spring Meeting, p. 26

Reiter, M., and Mansure, A. J., 1983, Geothermal studies in the San Juan basin and the Four Corners area of the Colorado Plateau I. Terrestrial heat flow measurements: Tectonophysics, v. 93, p. 233-251.

Reiter, M., Shearer, C., and Edwards, C. L., 1978, Geothermal anomalies along the Rio Grande rift in New Mexico: Geology, v. 6, p. 85-88

Ross, H. P., and Witcher, J. C., 1992, Self-potential expression of hydrothermal resources in the southern Rio Grande rift, New Mexico: Transactions, Geothermal Resources Council, v. 16, p. 247-253.

Sanford, R. M., Bowers, R. L., and Combs, J., 1979, Rio Grande rift geothermal exploration case history: Elephant Butte prospect, south-central New New Mexico: Transactions, Geothermal Resources Council, v. 3, p. 609-612.

Schoenmackers, R., 1988, Design and construction of the NMSU geothermally heated greenhouse research facility: New Mexico Research and Development Institute Report NMRDI 2-72-4214, 38 p.

Seager, W. R., Shafiqullah, M., Hawley, J. W., and Marvin, R. F., 1984, New KAr dates from basalts and the evolution of the southern Rio Grande rift: Geological Society of America Bulletin, v. 95, p. 87-99.

Shevenell, L., Goff; F., Vuataz, F., Trujillo, P. E., Counce, D., Janik, C. J., and Evans, W., 1987, Hydrogeological data for thermal and non thermal waters and gases of the Valles caldera southern Jemez Mountains region, New Mexico: Los Alamos National Laboratory Report LA-10923 OBES, $100 \mathrm{p}$.

Sinno, Y. A., Daggett, P. H., Keller, G. R., Morgan, P., and Harder, S. H., 1986, Crustal structure of the southern Rio Grande rift determined from seismic refraction profiling: Journal of Geophysical Research, v. 91 , no. B6, p. 61436156. 
Summers, W. K., 1976, Catalog of thermal waters in New Mexico: New Mexico Bureau of Mines and Mineral Reosurces Hydrologic Report 4, $80 \mathrm{p}$.

Swanberg, C. A., 1975, Detection of geothermal components in groundwaters of Dona Ana County, southern Rio Grande rift, New Mexico: New Mexico Geological Society 26th Field Conference Guidebook, p. 175-180.

Swanberg, C. A., 1980a, Chemistry, origin, and geothermal potential of thermal and non-thermal groundwaters in New Mexico: unpublished report submitted under USGS grant 14-08-001-6-255, $99 \mathrm{p}$.

Swanberg, C. A., 1980b, Estimated subsurface temperatures, in Icerman, L., Starkey, A., and Trentman, N., eds., State-Coupled Low-Temperature Geothermal Resource Assessment Program, Fiscal Year 1979: New Mexico Energy Institute at New Mexico State University, p. 1-1 to 1-17.

Swanberg, C. A., compiler, 1980c, Geothermal Resources of New Mexico: New Mexico Energy Institute at New Mexico State University in cooporation with the National Oceanic and Atmospheric Administration, 1:500,000 scale.

Swanberg, C. A., 1983, Geothermal resources of rifts: a comparison of the Rio Grande rift and the Salton trough: Tectonophysics, v. 94, p. 659-678.

Swanberg, C. A.and Icerman, L., compilers, 1983, Geothermal Resources of New Mexico, Scientific Map Series: New Mexico Energy Institute at New Mexico State University in cooporation with the National Oceanic and Atmospheric Administration, 1:500,000 scale.

Swanberg, C. A., 1984, Evaluation of the Na-K, Na-Li, K-Li, $\mathrm{SiO}_{2}$ (chalcedony), and $\mathrm{Na}-\mathrm{K}-\mathrm{Ca}$ geothermometers, in Icerman, L., ed., Regional Geothermal Exploration in North Central New Mexico: New Mexico Energy Research and Development Institute Report NMERDI 2-69-2208, p. 189-207.

Taylor, B., 1981, Heat flow studies and geothermal exploration in western TransPecos, Texas: unpublished PhD dissertation, University of Texas El Paso, 325 p. 
Wells, S. G., and Granzow, H., 1981, Hydrogeology of the thermal aquifer near Truth or Consequences, New Mexico, in Icerman, L., Starkey, A., Trentman, N., eds., State-Coupled Low-Temperature Geothermal Resource Assessment Program, Fiscal Year 1980, Final Technical Report: New Mexico Energy Institute at New Mexico State University, p. 3-5 to 3-35.

White, W. D., and Kelley, T. E.,1989, The San Andres-Glorieta Aquifer in westcentral New Mexico: New Mexico Geological Society 40th Field Conference Guidebook, p. 331-335.

Witcher, J. C., 1987, Geothermal resources in New Mexico - geologic settings and development update: The Interstate Oil and Gas Compact and Committee Bulletin, v. 1, no. 2, p. 49-60.

Witcher, J. C., 1988a, Geothermal resources of southwestern New Mexico and southeastern Arizona: New Mexico Geological Society 39th Field Conference Guidebook, p. 191-197.

Witcher, J. C., 1988b, Geothermal resources of the Jemez Pueblo, a prefeasibility assessment: Southwest Technology Development Institute, New Mexico State University, Technical Report prepared for the Council of Energy Resource Tribes, $50 \mathrm{p}$.

Witcher, J. C., 1990, Pre-drilling geophysical studies in the Indian Springs geothermal area, Jemez Pueblo, New Mexico: Southwest Technology Development Institute, New Mexico State University, Technical Report prepared for the Council of Energy Resource Tribes, $22 \mathrm{p}$.

Witcher, J. C., 1991a, The Rincon geothermal system, southern Rio Grande rift, New Mexico: a preliminary report on a recent discovery: Transactions, Geothermal Resources Council, v. 15, p. 205-212.

Witcher, J. C. 1991b, Jemez Pueblo geothermal assessment: Technology Enterprize Division, New Mexico Economic Development Department Report 278-5206, 11p. 
Witcher, J. C., Heller-Turrietta, M., Fischer, C., 1992, Geothermal resources at the Pueblo of Jemez: Southwest Technology Development Institute, New Mexico State University Technical Report prepared for the Council of Energy Resource Tribes, $36 \mathrm{p}$.

Witcher, J. C. and Schoenmackers, R., 1990, Time-integrated radon soil-gas surveys in geothermal exploration in the southern Rio Grande rift, New Mexico: Final Report to U. S. Department of Energy, Grant DE-FG07-88ID12794, 175 p.

Witcher, J. C., Whittier, J., and Morgan, R., 1990, New Mexico geothermal data base: Transactions, Geothermal Resources Council, v. 14 Part 1, p. 513-517 


\section{APPENDIX 1 \\ GEOTHERMAL SITES AND LOCATION DATA TABLES}

NOTES:

$\begin{array}{ll}\text { SITE ID } & \text { geothermal site number } \\ \text { NAME } & \text { well or spring name } \\ \text { WIS } & \text { well }- \text { w } / \text { spring }-s \\ \text { COUNTY } & \text { county name } \\ \text { DEPTH } & \text { well depth (meters) } \\ \text { TEMP } & \text { temperature }{ }^{\circ} \mathrm{C} \\ \text { LATITUDE } & \text { degrees } \\ \text { LONGITUDE } & \text { degrees } \\ \text { QUAD } & \text { quadrant of state (see Appendix } 6 \text { for description) } \\ \text { TWN } & \text { township (see Appendix } 6 \text { for description) } \\ \text { RNG } & \text { range (see Appendix } 6 \text { for description) } \\ \text { SEC } & \text { section (see Appendix } 6 \text { for description) } \\ \text { QTR } & \text { section quarters (see Appendix } 6 \text { for description) }\end{array}$




\begin{tabular}{|c|c|c|c|c|c|c|c|c|c|c|c|c|}
\hline SITEID & $\overline{\text { NAME }}$ & $W / S$ & COUNTY & $\begin{array}{c}\text { DEPTH } \\
\mathbf{m}\end{array}$ & $\begin{array}{c}\text { TEMP } \\
C\end{array}$ & LATITUDE & LONGITUDE & QUAD & TWN & $R N G$ & SEC & QTR \\
\hline BE1 & well & $w$ & Bernalillo & 362.1 & 40.5 & 35.0797 & 106.7211 & 2 & 10 & $\overline{2}$ & 22 & 143 \\
\hline BE2 & well & $w$ & Bernalillo & 371.9 & 34.5 & 35.0786 & 106.7319 & 2 & 10 & 2 & 21 & 412 \\
\hline $\mathrm{BE3}$ & well & $w$ & Bernalillo & 373.1 & 33 & 35.0803 & 106.8253 & 2 & 10 & 1 & 22 & 322 \\
\hline BE4 & West Mesa \#1 & $w$ & Bernalillo & & 32.2 & 35.0750 & 106.7367 & & & & & \\
\hline BE5 & West Mesa \#4 & $w$ & Bernalillo & & 32.1 & 35.0800 & 106.7233 & & & & & \\
\hline BE6 & well & $w$ & Bernalillo & 387.4 & 32 & 35.0717 & 106.6894 & 2 & 10 & 2 & 25 & 111 \\
\hline BE7 & Don \#1 & $w$ & Bermatillo & 490 & 31.4 & 35.0683 & 106.7517 & & & & & \\
\hline BE8 & well & $w$ & Bernalillo & 359.7 & 31 & 35.0722 & 106.7414 & 2 & 10 & 2 & 21 & 343 \\
\hline BE9 & West Mesa \#2 & $w$ & Bernalillo & & 30.6 & 35.0850 & 106.7317 & & & & & \\
\hline BE10 & well & $w$ & Bernalillo & & 30 & 35.0522 & 106.7261 & 2 & 10 & 2 & 33 & 240 \\
\hline BE11 & well & $w$ & Bernalillo & & 30 & 35.0814 & 106.7483 & 2 & 10 & 2 & 28 & 212 \\
\hline BE12 & well & $w$ & Bernalillo & & 30 & 35.0853 & 106.7236 & 2 & 10 & 3 & 21 & 213 \\
\hline BE13 & College \#2 & $w$ & Bernalillo & 480.1 & 30 & 35.1033 & 106.7358 & & & & & \\
\hline BE14 & well & $w$ & Bernalilio & & 29.0 & 35.1183 & 106.7100 & 2 & 10 & 2 & 15 & \\
\hline CA1 & hof spring & $\mathrm{s}$ & Catron & & 64.8 & 33.2333 & 108.2367 & 3 & 12 & 14 & 24 & 44 \\
\hline CA2 & hot spring & s & Catron & & 60.6 & 33.2333 & 108.2417 & 3 & 12 & 14 & 24 & 411 \\
\hline CA3 & Lower Frisco Hot Spring & s & Catron & & 46.11 & 33.2450 & 108.8817 & 3 & 12 & 20 & 23 & 12 \\
\hline CA4 & Lower Frisco Hot Spring & s & Catron & & 43.3 & 33.2450 & 108.8817 & 3 & 12 & 20 & 23 & 32 \\
\hline CA5 & Lower Frisco Hot Spring & $\mathbf{s}$ & Catron & & 43 & 33.2447 & 108.8811 & 3 & 12 & 20 & 23 & 321 \\
\hline CA6 & Lower Frisco Hot Spring & s & Catron & & 40.0 & 33.2467 & 108.8783 & 3 & 12 & 20 & 23 & 14 \\
\hline CA7 & Upper Frisco Hot Spring & s & Catron & & 36.5 & 33.8314 & 108.7994 & 3 & 5 & 19 & 35 & 132 \\
\hline CAB & Gila Middle Fork Hot Spring & s & Catron & & 36.0 & 33.2900 & 108.2650 & 3 & 11 & 14 & 34 & 24 \\
\hline CA9 & Lower Frisco Hot Spring & s & Catron & & 35 & 33.2447 & 108.8811 & 3 & 12 & 20 & 23 & 321 \\
\hline CA10 & Pueblo Windmill & $w$ & Catron & 320 & 33.8 & 34.5389 & 108.7772 & 1 & 4 & 19 & 25 & 414 \\
\hline CA11 & Frieborn Canyon Hot Spring & $\mathbf{s}$ & Catron & & 33.33 & 33.7100 & 109.0100 & 3 & 7 & 21 & 9 & 442 \\
\hline CA12 & hot spring & $\mathbf{s}$ & Catron & & 32.8 & 33.2600 & 108.2300 & 3 & 12 & 13 & 7 & 34 \\
\hline CA13 & test well & $w$ & Catron & 182.9 & 32.2 & 33.2200 & 108.2200 & 3 & 12 & 13 & 30 & 231 \\
\hline CA14 & well & $w$ & Catron & & 32 & 33.9575 & 107.8014 & $\frac{5}{3}$ & 4 & 9 & 17 & 311 \\
\hline CA15 & well & $w$ & Catron & 182.9 & 32 & 33.2250 & 108.2417 & 3 & 12 & 14 & 25 & 231 \\
\hline CA16 & Gila Middle Fork Hot Spring & $\mathbf{s}$ & Catron & & 31.0 & 33.2833 & 108.2633 & 3 & 11 & 14 & 35 & 34 \\
\hline CA17 & well & $w$ & Catron & 411.5 & 28 & 34.5400 & 108.8300 & 1 & 4 & 19 & 28 & 234 \\
\hline CA18 & Gila Middle Fork Meadows HS & s & Catron & & 27.5 & 33.3100 & 108.3300 & 3 & 11 & 14 & 30 & 2 \\
\hline CA19 & warm seep & s & Catron & & 27.2 & 33.2900 & 108.2600 & 3 & 11 & 14 & 35 & 4 \\
\hline $\mathrm{CA} 20$ & Zuni Salt Lake Warm Spring & $\mathbf{s}$ & Catron & & 26.0 & 34.4550 & 108.7667 & 1 & 3 & 18 & 30 & 31 \\
\hline CA21 & Gila Middle Fork Pool HS & $\mathbf{s}$ & Catron & & & 33.2333 & 108.2333 & 3 & 12 & 13 & 31 & $\frac{1}{1}$ \\
\hline CA22 & Glla Middle Fork Hot Spring & $\mathbf{s}$ & Catron & & 37 & 33.2833 & 108.2666 & 3 & 11 & 14 & 35 & 32 \\
\hline CA23 & Gila Middle Fork Hot Spring & s & Catron & & 34 & 33.2666 & 108.2500 & 3 & 12 & 14 & 1 & 33 \\
\hline $\mathrm{CB2}$ & well & $w$ & Cibola & 884.2 & 52.8 & 35.0644 & 107.7406 & 1 & 10 & 9 & 25 & 3241 \\
\hline $\mathrm{CB} 3$ & Acoma \#1 Well & $w$ & Cibola & 766.3 & 41.5 & 34.9806 & 107.7983 & 1 & 9 & 9 & 28 & 1344 \\
\hline CB4 & well & $w$ & Cibola & 615.4 & 41 & 34.6208 & 107.6542 & 1 & 5 & 8 & 35 & 123 \\
\hline $\mathrm{CB5}$ & well & $w$ & Cibola & 807.7 & 38.1 & 34.8914 & 107.6278 & 1 & 8 & 8 & 25 & 4231 \\
\hline CB6 & well & $w$ & Cibola & 984.2 & 36 & 34.8931 & 108.4147 & 1 & 8 & 15 & 27 & 311 \\
\hline CB7 & well & $w$ & Cibola & 807.7 & 34.5 & 35.0147 & 107.4836 & 1 & 9 & 6 & 16 & 111 \\
\hline CBB & well & $w$ & Cibola & 848.3 & 34 & 34.7625 & 107.9356 & 1 & 6 & 10 & 7 & 1413 \\
\hline CB9 & well & $w$ & Cibola & 524.3 & 34 & 35.0189 & 107.3097 & 1 & 9 & 5 & 12 & 4424 \\
\hline CB10 & well & $w$ & Cibola & 671.5 & 33.1 & 34.9005 & 107.4992 & 1 & 8 & 6 & 20 & 3334 \\
\hline CB12 & well & $w$ & Cibola & & 32 & 35.0939 & 108.4383 & 1 & 10 & 15 & 17 & 414 \\
\hline CB13 & Salado Spring & $s$ & Cibola & & 25 & 34.6900 & 107.4900 & 1 & 5 & 6 & 5 & 42 \\
\hline CV1 & well & $w$ & Chaves & & 33 & 33.0500 & 104.6808 & 4 & 15 & 23 & 6 & 22 \\
\hline DA1 & Chaffee 55-25 & $w$ & Dona Ana & 806.2 & 68 & 32.2781 & 106.6889 & 4 & 23 & 2 & 25 & 4111 \\
\hline DA2 & Radium Hot Springs Balley Well \#15 & $w$ & Dona Ana & & 70.0 & 32.4957 & 106.9167 & 3 & 21 & 1 & 10 & \\
\hline DA3 & Clary and Ruther State 1 & $w$ & Dona Ana & 784.3 & 69.4 & 32.2717 & 106.6981 & 4 & 23 & 2 & 36 & 1111 \\
\hline DA4 & Chaffee 35-25 & $w$ & Dona Ana & 196.6 & 68 & 32.2667 & 106.6833 & 4 & 23 & 2 & 25 & 123 \\
\hline DA5 & $L C-2$ & $w$ & Dona Ana & 269.8 & 67.9 & 32.3333 & 106.7000 & 4 & 23 & 2 & 34 & \\
\hline DA6 & Exxon Beard Ole Federal & $w$ & Dona Ana & 1219.5 & 65.5 & 32.3236 & 106.7128 & 4 & 23 & 2 & 11 & 1341 \\
\hline DA7 & Chaffee 12-24 & $w$ & Dona Ana & 400.8 & 65 & 32.2833 & 106.6833 & 4 & 23 & 2 & 24 & 311 \\
\hline DAB & NMSU PG-3 & $w$ & Dona Ana & 265.2 & 63.3 & 32.2878 & 106.7158 & 4 & 23 & 2 & 22 & 4442 \\
\hline DA9 & Radium Hot Springs Bailoy's bathh & $w$ & Dona Ana & 6.1 & 60.8 & 32.4967 & 106.9167 & 3 & 21 & 1 & 10 & 213 \\
\hline DA10 & Certified Sand Well & $w$ & Dona Ana & 182.9 & 58.8 & 32.2833 & 106.6833 & 4 & 23 & 2 & 14 & 431 \\
\hline DA11 & $\mathrm{T}-14$ & $w$ & Dona Ana & 1833 & 54.4 & 32.3900 & 106.4200 & 4 & 22 & 5 & 15 & 221 \\
\hline DA12 & Radium Hot Springs Hotel Well \#2 & $w$ & Dona Ana & 10.4 & 52.5 & 32.5003 & 106.7503 & 3 & 21 & 1 & 10 & 213 \\
\hline DA13 & LC-1 & $w$ & Dona Ana & 239.3 & 52.5 & 32.3089 & 106.7222 & 4 & 23 & 2 & 15 & 2334 \\
\hline DA14 & Radium Hot Springs Roy Smith Well & $w$ & Dona Ana & & 50.0 & 32.4967 & 106.9167 & 3 & 21 & 1 & 10 & \\
\hline DA15 & NMSU PG-2 & $w$ & Dona Ana & 153.9 & 47.7 & 32.2831 & 106.7250 & 4 & 23 & 2 & 27 & 1243 \\
\hline DA16 & Pure Oil Federal " $H^{\prime \prime} 1$ & $w$ & Dona Ana & 207.3 & 45 & 31.9494 & 106.9994 & 3 & 28 & $\frac{2}{2}$ & 24 & 213 \\
\hline DA17 & Nations Well & $w$ & Dona Ana & 108 & 43 & 32.2689 & 106.7194 & 4 & 23 & 2 & 34 & 2233 \\
\hline DA18 & well & $w$ & Dona Ana & 64.6 & 43 & 32.2697 & 106.7217 & 4 & 23 & 2 & 34 & 214 \\
\hline
\end{tabular}




\begin{tabular}{|c|c|c|c|c|c|c|c|c|c|c|c|c|}
\hline SITEID & NAME & $W / S$ & COUNTY & $\begin{array}{c}\text { DEPTH } \\
\mathrm{m}\end{array}$ & $\begin{array}{c}\text { TEMP } \\
\mathrm{C} \\
\end{array}$ & LATITUDE & LONGITUDE & QUAD & $T W N$ & $R N$ & SEC & QTR \\
\hline DA19 & Husand Well & $w$ & \begin{tabular}{|l|} 
Dona Ana \\
\end{tabular} & 106 & 4 & 32.2500 & 106.7167 & $\overline{4}$ & 23 & $\overline{2}$ & $\overline{34}$ & 234 \\
\hline DA20 & well & $w$ & \begin{tabular}{|l|} 
Dona Ana \\
\end{tabular} & 471.8 & 42.5 & 31.9942 & 106.8489 & 4 & 27 & 1 & 4 & $121 \mathrm{~A}$ \\
\hline DA21 & Rowan well & $w$ & Dona Ana & 100.6 & 36.8 & 32.2500 & 106.7167 & 4 & 23 & 2 & 34 & \\
\hline DA22 & Tellyer Well & $w$ & \begin{tabular}{|l|} 
Dona Ana \\
\end{tabular} & 148.1 & 36.5 & 32.2633 & 106.7208 & 4 & 23 & 2 & 34 & 4123 \\
\hline DA23 & Running Indian Well & $w$ & Dona Ana & 320 & 36 & 32.2625 & 107.0144 & 3 & 23 & 2 & 35 & 411 \\
\hline DA24 & well & $\bar{w}$ & \begin{tabular}{|l|} 
Dona Ana \\
\end{tabular} & & 36.0 & 32.6333 & 107.0000 & & & & & \\
\hline DA25 & well & $w$ & \begin{tabular}{|l|} 
Dona Anaa \\
\end{tabular} & 219.5 & 35.56 & 32.0667 & 106.6139 & 4 & 26 & 3 & 2 & \\
\hline DA26 & NMSU GD-1 & $w$ & \begin{tabular}{|l|} 
Dona Ana \\
\end{tabular} & 185 & 35 & 32.2883 & 106.7325 & 4 & 23 & 2 & 21 & 4424 \\
\hline DA27 & White well & $w$ & \begin{tabular}{|l|} 
Dona Ana \\
\end{tabular} & 94.8 & 34.5 & 32.2500 & 106.7167 & 4 & 23 & 2 & 34 & \\
\hline DA28 & well & $w$ & \begin{tabular}{|l|} 
Dona Ana \\
\end{tabular} & 59.4 & 34 & 32.5514 & 107.2922 & 3 & 20 & 4 & 19 & 324 \\
\hline DA29 & well & $w$ & Dona Ana & 131.1 & 33.5 & 32.0525 & 106.8711 & 4 & 26 & 1 & 18 & 222 \\
\hline DA30 & well & $w$ & \begin{tabular}{|l|} 
Dona Ana \\
\end{tabular} & 213.4 & 33.3 & 31.9394 & 106.6547 & 4 & 27 & 3 & 20 & 432 \\
\hline DA31 & Gardner Well & $w$ & \begin{tabular}{|l|} 
Dona Ana \\
\end{tabular} & 171.6 & 33 & 32.0100 & 106.0900 & 3 & 26 & 1 & 25 & 414 \\
\hline DA32 & $T-15$ & $w$ & \begin{tabular}{|l|} 
Dona Ana \\
\end{tabular} & 623 & 33 & 32.3500 & 106.4300 & 4 & 22 & 5 & 33 & 244 \\
\hline DA33 & well & $w$ & \begin{tabular}{|l|} 
Dona Ana \\
\end{tabular} & 365.8 & 32.22 & 32.2700 & 106.9800 & 3 & $23 \mid$ & 1 & 31 & 432 \\
\hline DA34 & well & $w$ & \begin{tabular}{|l|} 
Dona Ana \\
\end{tabular} & 243.8 & 32 & 32.2583 & 107.7833 & 3 & 23 & 2 & 35 & 430 \\
\hline DA35 & $T-17$ & $w$ & \begin{tabular}{|l|} 
Dona Ana \\
\end{tabular} & 765 & 32 & 32.2800 & 106.4100 & 4 & 23 & 5 & 27 & 142 \\
\hline DA36 & Berino Well & $w$ & \begin{tabular}{|l|} 
Dona Ana \\
\end{tabular} & \begin{tabular}{|l|}
152.7 \\
\end{tabular} & 31.1 & 32.0675 & 106.6147 & 4 & 26 & 3 & 11 & 111 \\
\hline DA37 & Souse Springs & $s$ & Dona Ana & & 31.0 & 32.6067 & 107.1950 & 3 & 19 & 3 & 31 & 34 \\
\hline DA38 & Test Hole & $w$ & \begin{tabular}{|l|} 
Dona Ana \\
\end{tabular} & & 31 & 32.1300 & 107.1600 & 3 & 25 & 3 & 17 & 111 \\
\hline DA39 & well & $w$ & \begin{tabular}{|l|} 
Dona Ana \\
\end{tabular} & & 31 & 32.1394 & 106.6647 & 4 & 25 & 1 & 17 & 111 \\
\hline DA40 & well & $w$ & \begin{tabular}{|l|} 
Dona Ana \\
\end{tabular} & & 31 & 32.0047 & 106.6097 & 4 & 26 & 3 & 35 & 141 \\
\hline DA41 & well & $w$ & \begin{tabular}{|l|} 
Dona Ana \\
\end{tabular} & 304.5 & 31 & 31.8256 & 106.8261 & 4 & 28 & 1 & 34 & 414 \\
\hline DA42 & well & $w$ & \begin{tabular}{|l|} 
Dona Ana \\
\end{tabular} & 306 & 31 & 32.8236 & 106.6319 & 4 & 28 & 3 & 34 & 331 \\
\hline DA43 & Pol Ranch Windmill & $w$ & \begin{tabular}{|l|} 
Dona Ana \\
\end{tabular} & & 30.5 & 32.0183 & 107.2617 & 3 & 26 & 4 & 28 & 131 \\
\hline DA44 & $\mathrm{sc2}$ & $w$ & \begin{tabular}{|l|} 
Dona Ana \\
\end{tabular} & & 30.5 & 32.2842 & 106.4314 & 4 & 23 & 5 & 28 & 223 \\
\hline DA45 & well & $w$ & \begin{tabular}{|l|} 
Dona Ana \\
\end{tabular} & \begin{tabular}{|l|}
549.3 \\
\end{tabular} & 30.5 & 31.9556 & 106.6989 & 4 & 27 & 2 & 13 & 331 \\
\hline DA46 & $T-18$ & $w$ & \begin{tabular}{|l|} 
Dona Ana \\
\end{tabular} & 72.8 & 30 & 32.3361 & 106.4575 & 4 & 23 & 5 & 5 & 321 \\
\hline DA47 & Dominguez Brothers Well & $w$ & Dona Ana & 76.2 & 30 & 32.1522 & 106.6544 & 4 & 25 & 3 & 8 & 214 \\
\hline DA48 & well & $w$ & Dona Ana & & 28.0 & 32.1533 & 106.9900 & 3 & 25 & 1 & 7 & 11 \\
\hline DA49 & Railroad Well & $w$ & Dona Ana & & & 32.6800 & 107.0300 & 3 & 19 & 2 & 9 & 12 \\
\hline DA50 & Radium Springs College Ranch Windmill & $w$ & Dona Ana & & & 32.5383 & 106.9394 & 3 & 20 & 1 & 27 & 34 \\
\hline DA51 & Radium Hot Springs Hotel Well \#1 & $\mathbf{w}$ & \begin{tabular}{|l|} 
Dona Ana \\
\end{tabular} & 3.7 & & 32.4967 & 167 & 3 & 21 & 1 & 10 & 213 \\
\hline DA52 & Radium Springs Masson 21 & $w$ & \begin{tabular}{|l|} 
Dona Ana \\
\end{tabular} & 85.3 & 76.1 & 32.5000 & 106 & 3 & 21 & 1 & 10 & 211 \\
\hline DA53 & Radium Springs Masson 22 & $w$ & \begin{tabular}{|l|} 
Dona Ana \\
\end{tabular} & & & 32.5000 & 106.9167 & 3 & 21 & 1 & 10 & 111 \\
\hline DA54 & Radium Springs Masson 23 & $w$ & \begin{tabular}{|l|} 
Dona Ana \\
\end{tabular} & & & 32.5000 & 100 & 3 & 21 & 1 & 10 & 112 \\
\hline DA55 & Radium Springs Masson 26 & $w$ & \begin{tabular}{|l|} 
Dona Ana \\
\end{tabular} & 36.6 & 76.7 & 32.5000 & & 3 & 21 & 1 & 10 & 211 \\
\hline DA56 & Radium Springs Ryan 72-35 & $w$ & \begin{tabular}{|l|} 
Dona Ana \\
\end{tabular} & 20.7 & 51.7 & 32.5000 & 106.9167 & 3 & 21 & 1 & 10 & 223 \\
\hline DA57 & NMSU GD-2 & $w$ & Dona Ana & 302 & & 32.2833 & & 4 & 23 & 2 & 27 & 1133 \\
\hline DA58 & NMSU OW-1 & $w$ & Dona Ana & \begin{tabular}{|l|}
262.1 \\
\end{tabular} & & 32.2839 & & 4 & 23 & 2 & 27 & 2242 \\
\hline DA59 & NMSU PG-1 & $w$ & Dona Ana & 262.1 & & 32.2842 & 106.7170 & 4 & 23 & 2 & 27 & 2241 \\
\hline DA60 & NMSU PG-4 & $w$ & Dona Ara & 309.4 & & 32.2 & & 4 & 23 & 2 & 23 & 3314 \\
\hline DA61 & Radium Springs Masson 25 (injection) & $w$ & \begin{tabular}{|l|} 
Dona Ana \\
\end{tabular} & 62.8 & & 32.5000 & & 3 & 21 & 1 & 10 & \\
\hline DA62 & Radium Springs Masson 27 (injection) & $w$ & \begin{tabular}{|l|} 
Dona Ana \\
\end{tabular} & 24.4 & & 32.5000 & 106 & 3 & 21 & 1 & 10 & \\
\hline DA63 & Radium Springs Masson 28 & $w$ & \begin{tabular}{|l|} 
Dona Ana \\
\end{tabular} & 36.6 & 71.7 & 32.5 & & 3 & 21 & 1 & 10 & 211 \\
\hline ED1 & Clayton Well & $w$ & Eddy & & 31 & 32.5603 & 104. & 4 & 20 & 27 & 21 & 133 \\
\hline GR1 & Miimbres Hot Springs \#3 & $\mathbf{s}$ & Grant & & & 32.7483 & 107 & 3 & 18 & 10 & 13 & 11 \\
\hline GR2 & Gila Hot Springs Doyle Well & $w$ & Grant & \begin{tabular}{|l|}
138.7 \\
\end{tabular} & 74 & 33.1967 & 108.2035 & 3 & 13 & 13 & 5 & 2323 \\
\hline GR3 & Turkey Creek Hot Spring & $\mathbf{s}$ & Grant & & 74.0 & 33.108 & 108.4833 & 3 & 14 & 16 & 3 & 34 \\
\hline GR4 & Gila Hot Springs Campbell \#4 Well & $w$ & Grant & 72.5 & 72 & 33.198 & 108 & 3 & 13 & 13 & 5 & 2141 \\
\hline GR5 & Gila Spring Hot Spring \#14 & $\mathbf{s}$ & Grant & & 66 & 33.20 & 108.2000 & 3 & 13 & 13 & 5 & 2 \\
\hline GR6 & Gila HS northern artesian well & $\mathbf{w}$ & Grant & & 65 & 33.2 & 108.2083 & 3 & 13 & 13 & 5 & 12 \\
\hline GR7 & Gila Hot Springs & 5 & Grant & & 64 & 33.1 & 108. & 3 & 13 & 13 & 5 & 214 \\
\hline GR8 & well & $w$ & Grant & 158.5 & 62.2 & 32.670 & 108. & 3 & 19 & 12 & 12 & \\
\hline GR9 & Gila HS middle artesian well & $w$ & Grant & & 62 & 33.2000 & & 3 & 13 & 13 & 5 & 12 \\
\hline GR10 & Gila Hot Springs Campbell \#2 Well & $w$ & Grant & 84.4 & 61 & 33.2000 & 108 & 3 & 13 & 13 & 5 & 212 \\
\hline GR11 & Miimbres Hot Springs \#25 & $s$ & Grant & & 59 & 32.7483 & 107.8350 & 3 & 18 & 10 & 13 & 11 \\
\hline GR12 & Miimbres Hot Springs \#28 & 5 & Grant & & 58.06 & 32.7483 & 107.8350 & 3 & 18 & 10 & 13 & 11 \\
\hline GR13 & Gilla Spring Hot Spring \#41 & $\mathbf{s}$ & Grant & & 58 & 33.2 & 108.2000 & 3 & 13 & 13 & 5 & 2 \\
\hline GR14 & Mimbres Hot Springs & $s$ & Grant & & 58 & 32.7492 & 107.8344 & 3 & 18 & 1 & 13 & 111 \\
\hline GR15 & Milimbres Hot Springs \#8 & $\mathbf{s}$ & Grant & & 57.5 & 32.7483 & 107.8350 & 3 & 18 & 10 & 13 & 11 \\
\hline GR16 & Miimbres Hot Springs \#12 & $\mathbf{s}$ & Grant & & 56.4 & 32.7483 & 107.8350 & 3 & 18 & 10 & 13 & 11 \\
\hline GR17 & Milimbres Hot Springs \#21 & $s$ & Grant & & 55 & 32.7483 & 107.8350 & 3 & 18 & 10 & 13 & 11 \\
\hline GR18 & Miimbres Hot Springs \#23 & $\mathbf{s}$ & Grant & & 54.44 & 32.7483 & 107.8350 & 3 & 18 & 10 & 13 & 11 \\
\hline GR19 & Faywood Hot Springs & $\mathbf{s}$ & Grant & & 53 & 32.5547 & 107.9950 & 3 & 20 & 11 & 20 & 243 \\
\hline GR20 & Gila Lyons Lodge swimming pool HS & 5 & Grant & & 52.2 & 33.1847 & 108.1744 & 3 & 13 & 13 & 10 & 121 \\
\hline GR21 & well & $w$ & Grant & & 52 & 33.1847 & 108.1744 & 3 & 13 & 13 & 10 & 121 \\
\hline
\end{tabular}




\begin{tabular}{|c|c|c|c|c|c|c|c|c|c|c|c|c|}
\hline SITEID & NAME & $W / S$ & COUNTY & $\begin{array}{c}D E P T H \\
m\end{array}$ & \begin{tabular}{|c|}
$T E M P$ \\
$C$
\end{tabular} & LATITUDE & LONGITUDE & QUAD & $T W N$ & RNG & SEC & QTR \\
\hline GR22 & Gila River Waterfall HS & 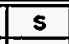 & Grant & & 43.6 & 33.1633 & 108.1833 & 3 & 13 & 13 & 17 & 32 \\
\hline GR23 & Gila East Fork no name spring & s & Grant & & 41.39 & 33.1833 & 108.1667 & 3 & 13 & 13 & 10 & 2 \\
\hline GR24 & Kennecott Warms Springs Well & $w$ & Grant & 36.6 & 41.1 & 32.5700 & 108.0200 & 3 & 20 & 11 & 18 & 3133 \\
\hline GR25 & Gila East Fork Hot Spring & $\mathbf{s}$ & Grant & & 36.0 & 33.1917 & 108.1833 & 3 & 13 & 13 & 10 & 121 \\
\hline GR26 & Gila Lyons Lodge East Fork Hot Springs & s & Grant & & 35.56 & 33.1847 & 108.1744 & 3 & 13 & 13 & 10 & 121 \\
\hline GR27 & Kennecott Warm Spring Well \#3 & $w$ & Grant & 68 & 34.55 & 32.5700 & 108.0200 & 3 & 20 & 11 & 18 & 310 \\
\hline GR28 & Kennecott Warms Springs Well \#2 & $w$ & Grant & 13.4 & 34.4 & 32.5700 & 108.0200 & 3 & 20 & 11 & 18 & 31434 \\
\hline GR29 & Kennecott Warms Springs Well \#3 & $w$ & Grant & 68 & 34.4 & 32.5700 & 108.0200 & 3 & 20 & 11 & 18 & 33221 \\
\hline GR30 & Cliff Warm Well & $w$ & Grant & 91.4 & 33.5 & 32.9758 & 108.5931 & 3 & 15 & 17 & 27 & 111 \\
\hline GR31 & Kennecott Warm Springs Well \#1 & $w$ & Grant & 7.6 & 33.3 & 32.5700 & 108.0200 & 3 & 20 & 11 & 18 & 332 \\
\hline GR32 & Muir Ranch well & $w$ & Grant & & 33.0 & 32.2283 & 108.5117 & 3 & 24 & 16 & 8 & 32 \\
\hline GR33 & Ground Hog Mine & $w$ & Grant & 670 & 32 & 32.7800 & 108.0100 & 3 & 17 & 12 & 32 & 44414 \\
\hline GR34 & well & $w$ & Grant & 609.6 & 32 & 32.7797 & 108.0994 & & & & & $\cdot$ \\
\hline GR35 & well & $w$ & Grant & 268.5 & 31.1 & 32.6444 & 108.1278 & & & & & \\
\hline GR36 & Spring Canyon Warm Spring & $\mathbf{s}$ & Grant & & 31.0 & 32.8767 & 108.5833 & 3 & 16 & 17 & 34 & 21 \\
\hline GR37 & Apache Tejo Warm Springs Well \#7 & $w$ & Grant & 268.5 & 31 & 32.6444 & 108.1278 & 3 & 19 & 12 & 19 & 13212 \\
\hline GR38 & Kennecott Warms Springs Well \#4 & $w$ & Grant & 89 & 30.5 & 32.5700 & 108.0200 & 3 & 20 & 11 & 18 & 133 \\
\hline GR39 & Riverside Well & $w$ & Grant & 10.9 & 30 & 32.9300 & 108.0600 & 3 & 16 & 17 & 9 & 242 \\
\hline GR40 & Kennecolt Warms Springs Well \#6 & $w$ & Grant & 121.9 & 30 & 32.5500 & 108.0200 & 3 & 20 & 11 & 19 & 11133 \\
\hline GR41 & Mangas Springs & $\mathbf{s}$ & Grant & & 27.0 & 32.8417 & 108.5100 & 3 & 17 & 16 & 8 & 24 \\
\hline GR42 & Allen Spring & $\mathbf{s}$ & Grant & & 25.6 & 32.3617 & 108.3617 & 3 & 16 & 15 & 26 & 41 \\
\hline GR43 & Cliff Warm Spring & $\mathbf{s}$ & Grant & & 25 & 32.9750 & 108.6250 & 3 & 15 & 17 & 30 & 222 \\
\hline GR44 & Apache Tejo Warms Springs Well \#4 & $w$ & Grant & 236.8 & & 32.6400 & 108.1200 & 3 & 19 & 12 & 19 & 3 \\
\hline GR45 & Apache Tejo Warms Springs Well & $w$ & Grant & 745.2 & & 32.6400 & 108.1100 & 3 & 19 & 12 & 19 & 3 \\
\hline HD1 & Lightning Dock Burgett Well \#1 & $w$ & Hidalgo & & 104.0 & 32.1450 & 108.8317 & 3 & 25 & 19 & 7 & \\
\hline HD2 & Lightning Dock hot well & $w$ & Hidalgo & & 98 & 32.1458 & 108.8325 & 3 & 25 & 19 & 7 & $234 \mathrm{~A}$ \\
\hline HD3 & Lightning Dock hot well & $\mathbf{w}$ & Hidalgo & & 96 & 32.1450 & 108.8317 & 3 & 25 & 19 & 7 & 134 \\
\hline HD4 & Lightning Dock hot well & $w$ & Hidalgo & & 95.5 & 32.1458 & 108.8325 & 3 & 25 & 19 & 7 & 234 \\
\hline HDS & Lighting Dock Burgett Well \#10 & $w$ & Hidalgo & & 95.2 & 32.1450 & 108.8317 & 3 & 25 & 19 & 7 & \\
\hline HD6 & Lightning Dock hot well & $w$ & Hidalgo & & 94 & 32.1486 & 108.8314 & 3 & 25 & 19 & 7 & \\
\hline HD7 & Lightning Dock hot well & $w$ & Hidalgo & & 85.0 & 32.1450 & 108.8317 & 3 & 25 & 19 & 7 & 23 \\
\hline HD8 & Lightning Dock McCants Well & $w$ & Hidalgo & & 81.0 & 32.1483 & 108.8317 & 3 & 25 & 19 & 7 & 21 \\
\hline HD9 & Lightning Dock hot well & $w$ & Hidalgo & & 71.0 & 32.1450 & 108.8400 & 3 & 25 & 19 & 7 & 14 \\
\hline HD10 & Lightning Dock hot well & $w$ & Hidalgo & 36.6 & 52 & 32.1453 & 108.8389 & 3 & 25 & 19 & 7 & 143 \\
\hline HD11 & warm well & $w$ & Hidalgo & & 35 & 32.4167 & 108.9833 & 3 & 22 & 21 & 3 & 312 \\
\hline HD12 & Lightning Dock hot well & $w$ & Hidalgo & 86.3 & 33.5 & 32.1453 & 108.8425 & 3 & 25 & 19 & 7 & $\overline{133}$ \\
\hline HD13 & well & $w$ & Hidalgo & 734.6 & 32 & 31.7125 & 108.3228 & 3 & 30 & 15 & 12 & 323 \\
\hline HD14 & Blowing Well & $w$ & Hidalgo & 136.9 & 31 & 32.4178 & 108.9928 & 3 & 22 & 21 & 3 & 3234 \\
\hline HD15 & well & $w$ & Hidalgo & 169.8 & 30 & 32.4433 & 109.0331 & 3 & 21 & 21 & 30 & 444 \\
\hline HD16 & Kipp Ranch Hot Well & $w$ & Hidalgo & & & 32.3100 & 108.5700 & 3 & 23 & 17 & 10 & 11 \\
\hline HD17 & Lightning Dock hot well & $w$ & Hidalgo & & & 32.1450 & 108.8317 & 3 & 25 & 19 & 7 & 243 \\
\hline HD18 & Lightning Dock hot well & $w$ & Hidalgo & & & 32.1450 & 108.8317 & 3 & 25 & 19 & 7 & $234 b$ \\
\hline HD19 & Lightning Dock hot well & $w$ & Hidalgo & & & 32.1450 & 108.8317 & 3 & 25 & 19 & 7 & 342 \\
\hline $\mathrm{HD} 20$ & Lightning Dock hot well & $w$ & Hidalgo & & & 32.1450 & 108.8317 & 3 & 25 & 19 & 7 & 243 \\
\hline HD21 & Lightning Dock Rosette 1 & $w$ & Hidalgo & 134.1 & 104.4 & 32.1300 & 108.8300 & 3 & 25 & 19 & 6 & 4 \\
\hline $\mathrm{HD} 22$ & Lightning Dock Rosette 1 & $w$ & Hidalgo & 170.7 & 107.2 & 32.1300 & 108.8300 & 3 & 25 & 19 & 6 & 4 \\
\hline $\mathrm{HD} 23$ & Lightning Dock Rosette 1 & $w$ & Hidalgo & 134.1 & 106.7 & 32.1300 & 108.8300 & 3 & 25 & 19 & 6 & 4 \\
\hline LA1 & Pueblo Canyon \#7 Well & $w$ & Los Alamos & & 31 & 35.8833 & 106.2636 & 2 & 19 & 6 & 13 & 131 \\
\hline LU1 & Smyer Well & $w$ & \begin{tabular}{l|} 
Luna \\
\end{tabular} & 338.3 & 39 & 32.2508 & 107.5881 & 3 & 24 & 7 & 5 & 133 \\
\hline LU2 & Nunn Stock Well & $w$ & Luna & & 38 & 32.5100 & 107.4900 & 3 & 21 & 6 & 6 & 11332 \\
\hline LU3 & well & $w$ & Luna & 129.5 & 34.5 & 32.2461 & 107.5506 & 3 & 24 & 7 & 3 & 300 \\
\hline LU4 & well & $w$ & Luna & 161.2 & 33 & 31.8500 & 107.6092 & 3 & 28 & 8 & 25 & 211 \\
\hline LU5 & well & $w$ & Luna & 199 & 32 & 31.8600 & 107.0600 & 3 & 28 & 7 & 19 & 2211 \\
\hline LU6 & well & $w$ & Luna & 304.8 & 31.5 & 32.1547 & 107.4828 & 3 & 25 & 6 & 8 & 112 \\
\hline LU7 & well & $w$ & Luna & & 31.5 & 31.8033 & 107.7867 & 3 & 29 & 9 & 8 & 11 \\
\hline LU8 & well & $w$ & Luna & & 31.1 & 31.8017 & 107.7817 & 3 & 29 & 9 & 8 & 23 \\
\hline LU9 & well & $w$ & Luna & 304.8 & 31 & 31.8861 & 107.5597 & 3 & 28 & 7 & 9 & 411 \\
\hline LU10 & well & $w$ & Luna & 213.4 & 31 & 31.8400 & 107.5300 & 3 & 28 & 7 & 26 & 4222 \\
\hline LU11 & well & $w$ & Luna & 142.6 & 31 & 31.8511 & 107.8683 & 3 & 29 & 10 & 9 & 3111 \\
\hline LU12 & well & $w$ & Luna & 335.3 & 30.7 & 31.8133 & 107.7900 & 3 & 29 & 9 & 8 & 12134 \\
\hline LU13 & Little Ed Well & $w$ & Luna & 65.8 & 30 & 32.3353 & 107.3492 & 3 & 23 & 5 & 3 & 311 \\
\hline LU14 & well & $w$ & Luna & & 30.0 & 31.8417 & 107.5200 & 3 & 28 & 7 & 26 & 42 \\
\hline LU15 & well & $w$ & Luna & 49.4 & 30 & 31.8439 & 107.6361 & 3 & 28 & 8 & 26 & 311 \\
\hline MK1 & Fort Wingate Well/Santa Fe 'Spring' & $w$ & McKinley & 592.3 & 55 & 35.4667 & 108.5744 & 1 & 15 & 16 & 30 & 3443 \\
\hline MK2 & well & $w$ & McKinley & 905.3 & 46 & 35.4244 & 107.8789 & 1 & 14 & 10 & 22 & 400 \\
\hline MK3 & Ya-Ta-Hey Well & $w$ & McKinley & & 45.5 & 35.6400 & 108.7800 & & & & & \\
\hline MK4 & well & $w$ & McKinley & & 42.2 & 35.9594 & 107.8981 & 1 & 20 & 10 & 16 & 4413 \\
\hline
\end{tabular}




\begin{tabular}{|c|c|c|c|c|c|c|c|c|c|c|c|c|}
\hline SITEID & NAME & W/S & COUNTY & \begin{tabular}{|c} 
DEPTH \\
$\mathbf{m}$
\end{tabular} & $\mid \begin{array}{c}\text { TEMP } \\
\mathrm{C}\end{array}$ & LATITUDE & LONGITUDE & QUAD & $T W N$ & RNG & SEC & QTR \\
\hline MK5 & well & $w$ & McKinley & 939.1 & 40.2 & 35.4256 & 107.8803 & 1 & 14 & 10 & 22 & $\overline{414}$ \\
\hline MK6 & well & $w$ & McKinley & 965.3 & 39 & 35.6289 & 108.7764 & 1 & 16 & 18 & 7 & 423 \\
\hline MK7 & Navajo well & $w$ & McKinley & 762 & 37.5 & 35.8525 & 108.6694 & & & & & \\
\hline MK8 & Toh Sah Toh & $w$ & McKinley & & 37.0 & 35.8700 & 108.6800 & & & & & \\
\hline MK9 & well & $w$ & McKinley & 981.5 & 36 & 35.6228 & 108.7683 & 1 & 16 & 18 & 17 & 122 \\
\hline \begin{tabular}{|l} 
MK10 \\
\end{tabular} & NTUA \#2Well & $w$ & McKinley & & 36 & 35.6953 & 108.1439 & 1 & 17 & 12 & 20 & 1111 \\
\hline MK11 & well & $w$ & McKinley & & 35.5 & 35.6842 & 108.1442 & 1 & 17 & 12 & 20 & 3313 \\
\hline MK12 & Pure Oil Navajo \#1 Well (Tohachi Wel) & $w$ & McKinley & 762 & 35 & 35.8511 & 108.6683 & 1 & 19 & 17 & 29 & 230 \\
\hline MK13 & Pure Oil Navajo $\$ 3$ Well & $w$ & McKinley & 243.8 & 35 & 35.8600 & 108.6400 & 1 & 19 & 17 & 22 & \\
\hline MK14 & Mobil Well Crown Point & $w$ & McKinley & 655.3 & 33.5 & 35.7008 & 108.2158 & 1 & 17 & 13 & 15 & 332 \\
\hline MK15 & well & $w$ & McKinley & & 33.5 & 35.9297 & 107.9778 & 1 & 20 & 11 & 26 & 3414 \\
\hline $\begin{array}{l}\text { MK16 } \\
\end{array}$ & well & $\bar{w}$ & McKinley & & 33 & 35.6847 & 108.1528 & 1 & 17 & 12 & 19 & 4314 \\
\hline \begin{tabular}{|l|} 
MK17 \\
\end{tabular} & well & $w$ & MoKinley & 654.7 & 32.5 & 35.6372 & 108.7917 & 1 & 16 & 18 & 7 & 1111 \\
\hline MK18 & well & $w$ & McKinley & & 32.5 & 35.9314 & 107.9397 & 1 & 20 & 10 & 30 & 3244 \\
\hline \begin{tabular}{|l|} 
MK19 \\
\end{tabular} & well & $w$ & McKinley & 502.9 & 32 & 35.5361 & 108.5878 & 1 & 15 & 17 & 13 & 210 \\
\hline MK20 & well & $w$ & McKinley & 240.2 & 32 & 35.6250 & 108.7917 & 1 & 16 & 18 & 7 & 3333 \\
\hline MK21 & well & $w$ & McKinley & 877.8 & 31.5 & 35.5683 & 108.7611 & 1 & 16 & 18 & 32 & 44 \\
\hline MK22 & well & $w$ & McKinley & & 30.5 & 35.0372 & 108.7611 & 1 & 9 & 18 & 5 & 324 \\
\hline MK23 & well & $w$ & McKinley & & 30.5 & 35.6522 & 108.0450 & 1 & 16 & 11 & 5 & 1212 \\
\hline MK24 & well & $w$ & MeKinley & & 30 & 35.6714 & 108.1561 & 1 & 17 & 12 & 30 & 3241 \\
\hline $0 T 1$ & N-9 Well & $w$ & Otero & 137 & 71.1 & 32.0931 & 106.1514 & 4 & 26 & 8 & 5 & 332 \\
\hline OT2 & N-11 Well & $w$ & Otero & 227 & 61.1 & 32.0842 & 106.1514 & 4 & 25 & 8 & 32 & 333 \\
\hline OT3 & M-11 Well & $w$ & Otero & 153 & 50 & 32.0789 & 106.1750 & 4 & 26 & 7 & 1 & 241 \\
\hline OT4 & FLOUR-1 Well & $w$ & Otero & 321.3 & 33.5 & 32.4306 & 811 & 4 & 21 & 7 & 36 & 344 \\
\hline OT5 & Garton Well & $w$ & Otero & 301.5 & 34 & 32.7800 & 106.1500 & 4 & 18 & 8 & 5 & 431 \\
\hline OT6 & N-8 Well & $w$ & Otero & 304 & & 32.0167 & 106.0833 & 4 & 26 & 8 & 33 & 120 \\
\hline OT7 & $\mathrm{N}-11$ & $w$ & Otero & 227.1 & 61 & 32.0841 & 106.1513 & 4 & 25 & 8 & 32 & 300 \\
\hline RA1 & Ojo Caliente Hot Spring Soda Spring & 5 & Rio Arriba & & & 36.3050 & 106.0567 & 2 & 24 & 8 & 24 & 11 \\
\hline RA2 & Ojo Caliente Hot Spring Well & $w$ & \begin{tabular}{|l|} 
Rio Arriba \\
\end{tabular} & 26.5 & 55.6 & 36.3050 & 567 & 2 & 24 & 8 & 24 & 11 \\
\hline RA3 & Ojo Callente Arsenic Hot Spring & $\mathbf{s}$ & Rio Arriba & & 45.0 & 36.3050 & 106.0567 & 2 & 24 & 8 & 24 & 11 \\
\hline RA4 & Ojo Caliente Hot Spring & s & Rlo Arriba & & 43 & 36.3050 & 106.0567 & 2 & 24 & 8 & 24 & 11 \\
\hline RA5 & Ojo Caliente HS Lithia Spring & s & Rio Arriba & & 41.9 & 36.3050 & 106.0567 & 2 & 24 & 8 & 24 & 11 \\
\hline RA6 & Ojo Caliente HS Sodium Sulfate Spring & $\mathbf{s}$ & Rio Arriba & & 40.6 & 36.3050 & 106.0567 & 2 & 24 & 8 & 24 & 11 \\
\hline RA7 & Ojo Caliente Hot Spring Iron Spring & s & Rio Arriba & & 40 & 36.3044 & 0522 & 2 & 24 & 8 & 24 & 11 \\
\hline RAB & Statue Spring & $\mathbf{s}$ & \begin{tabular}{|l|} 
Rio Arriba \\
\end{tabular} & & 36 & 36.3683 & 106.0583 & 2 & 25 & 8 & 26 & 414 \\
\hline RA9 & Ojo Caliente Hot Spring Well \#1 & $w$ & Rio Arriba & 14.6 & 34.3 & 36.3050 & 567 & 2 & 24 & 8 & 24 & 11 \\
\hline RA10 & spring & 5 & $\begin{array}{l}\text { Rio Arriba } \\
\end{array}$ & & 27.5 & 36.3667 & 450 & 2 & 25 & 8 & 25 & 44 \\
\hline RA11 & spring & s & Rio Arriba & & 25.5 & 36. & 17 & 2 & 25 & 8 & 36 & 22 \\
\hline RA12 & Ojo Caliente Hot Spring Well \#2 & $w$ & Rio Arriba & 16.2 & & 36.3050 & 67 & 2 & 24 & 8 & 24 & 11 \\
\hline SA1 & Jemez/Baca \# 15 & $w$ & Sandoval & & 326.0 & 35.8933 & 00 & & & & & \\
\hline SA2 & Jemez/Baca \# 4 & $\bar{w}$ & Sandoval & & 29 & 35.8892 & 106. & & & & & \\
\hline SA3 & Jemez/Baca \# 4 & $w$ & Sandoval & & 294.0 & 35.8892 & 106 & & & & & \\
\hline SA4 & Jemez/Baca \# 13 & $w$ & Sandoval & & 279.0 & 35.8964 & 106.5678 & & & & & \\
\hline SA5 & Jemez/Baca \#13 & $w$ & \begin{tabular}{|l|} 
Sandoval \\
\end{tabular} & & 278.0 & 35.8964 & 106. & & & & & \\
\hline SA6 & Jemez/Baca \#24 & $w$ & Sandoval & & 261.0 & 35.8844 & 106.5825 & & & & & \\
\hline SA7 & Jemez/GRIWC 23-4 at $6300 \mathrm{ft}$ depth & $w$ & Sandoval & & \begin{tabular}{|l|}
232.6 \\
\end{tabular} & 35.9094 & 106.6314 & & & & & \\
\hline SAB & Jemez/Baca \# 19 & $w$ & Sandoval & & 223.0 & 35.8933 & 106.5800 & & & & & \\
\hline SA9 & Jemez/GRI WC $23-4$ at $4800 \mathrm{ft}$ depth & $w$ & Sandoval & & 214.0 & 35.9094 & 106.6314 & & & & & \\
\hline SA10 & Jemez/Sulphur Springs Women's Bathhouse & $\mathbf{s}$ & Sandoval & & 90.0 & 35.9081 & 150 & & & & & \\
\hline SA11 & Jemez/Sulphur Springs main fumarole & $s$ & Sandoval & & 88.0 & 35.9081 & 50 & & & & & \\
\hline SA12 & Jémez HS Jemez Springs \#1 Well & $w$ & Sandoval & & 73.3 & 35.7733 & 889 & 2 & 18 & 2 & 23 & \\
\hline SA13 & Jemez HS Travertine Mound Spring & $s$ & Sandoval & & 72.6 & 35.7733 & 106.6889 & 2 & 18 & 2 & 23 & \\
\hline SA14 & Jemez HS Soda Spring & $\mathbf{s}$ & Sandoval & & 65.5 & 35.7725 & 106 & 2 & 18 & 2 & 23 & \\
\hline SA15 & Jemez/Sulphur Springs unnamed HS & $\mathbf{s}$ & Sandoval & & 63.0 & 35.9081 & 106.6150 & & & & & \\
\hline SA16 & Jemez Hot Springs & s & Sandoval & & 58 & 35.7706 & 106.6914 & 2 & 18 & 2 & 23 & 432 \\
\hline SA17 & Jemez/Sulphur Springs Lemonade Spring & s & Sandoval & & 58.0 & 35.9081 & 106.6150 & & & & & \\
\hline SA18 & Jemez Pueblo \#1 Well & $w$ & Sandoval & 73.2 & 57.8 & 35.5900 & 106.7530 & 2 & 16 & 2 & 29 & 213 \\
\hline SA19 & Jemez HS Gazebo Spring & s & Sandoval & & 55.0 & 35.7733 & 106.6889 & $\frac{5}{2}$ & 18 & 2 & 23 & \\
\hline SA2O & Kaseman \#2 Well (Zia Hot Well) & $w$ & Sandoval & 612.7 & 52 & 35.6456 & 106.8883 & $\frac{2}{1}$ & 16 & $\frac{2}{1}$ & $\frac{20}{1}$ & 4211 \\
\hline SA21 & Jemez HS Buddhist Spring & $s$ & Sandoval & & 50.0 & 35.7733 & 106.6889 & 2 & 18 & 2 & 23 & \\
\hline SA22 & Jemez HS Marsh Spring & s & Sandoval & & 49.0 & 35.7733 & 106.6889 & $\frac{5}{2}$ & 18 & 2 & 23 & \\
\hline SA23 & Jemez'Soda Dam Hot Spring & $\mathbf{s}$ & Sandoval & & 46 & 35.7914 & 106.6861 & 2 & 18 & 2 & 14 & 442 \\
\hline SA24 & Jemez'Soda Dam Hot Springs (west spring) & $\mathbf{s}$ & Sandoval & & 46 & 35.7917 & 106.6864 & 2 & 18 & 2 & 14 & \\
\hline SA25 & Jemez/Spence Hot Spring & s & Sandoval & & 44 & 35.8494 & 106.6283 & $\frac{5}{2}$ & 19 & 3 & 28 & 143 \\
\hline SA26 & Jemez/Sulphur Springs Men's Bathhouse & s & Sandoval & & 43.5 & 35.9067 & 106.6153 & & & & & \\
\hline SA27 & Jemez Pueblo Indian Hot Spring & s & Sandoval & & 43.0 & 35.5911 & 106.7531 & 2 & 16 & 2 & 29 & 1423 \\
\hline SA28 & Jemez/McCauley Hot Spring & s & Sandoval & & 43 & 35.8200 & 106.6267 & 2 & 18 & 3 & 4 & 321 \\
\hline
\end{tabular}




\begin{tabular}{|c|c|c|c|c|c|c|c|c|c|c|c|c|}
\hline SITEID & NAME & W/S & COUNTY & \begin{tabular}{|c|} 
DEPTH \\
$\mathbf{m}$
\end{tabular} & $\begin{array}{c}\text { TEMP } \\
C\end{array}$ & LATITUDE & LONGITUDE & $Q U A D$ & TWN & RNG & SEC & QTR \\
\hline$\overline{\text { SA29 }}$ & Jemez/San Antonio Hot Spring & $\bar{s}$ & Sandoval & & 41.3 & 35.9383 & 106.6456 & $\overline{2}$ & 20 & 3 & 29 & 1 \\
\hline SA30 & Jemez/Sulphur Springs Footbath Spring & s & Sandoval & & 40.5 & 35.9081 & 106.6150 & & & & & \\
\hline SA31 & Jemez/San Antonio Warm Springs & $\mathbf{s}$ & Sandoval & & 38.5 & 35.9711 & 106.5614 & & & & & \\
\hline SA32 & Jemez/Soda Dam Grotto Spring & $\mathbf{s}$ & Sandoval & & 38.0 & 35.7914 & 106.6864 & 2 & 18 & 2 & 13 & \\
\hline SA33 & Jemez/Bathhouse HS & $s$ & Sandoval & & 37.4 & 35.9714 & 106.5606 & & & & & \\
\hline SA34 & Jemez/Sulphur Springs Electric Spring & $\mathbf{s}$ & Sandoval & & 36.0 & 35.9081 & 106.6150 & & & & & \\
\hline SA35 & Jemez/Little Spence Hot Spring & $\mathbf{s}$ & Sandoval & & 34.0 & 35.8494 & 106.6289 & 2 & 19 & 3 & 28 & \\
\hline SA36 & Star Lake \#2 Ojo Encino Well & $w$ & Sandoval & & 33 & 36.0047 & 107.3775 & 1 & 21 & 5 & 32 & 424 \\
\hline SA37 & Jemez'Soda Dam Hidden Warm Spring & $\mathbf{s}$ & Sandoval & & 32.3 & 35.7914 & 106.6864 & 2 & 18 & 2 & 14 & \\
\hline SA38 & well & $w$ & Sandoval & & 32 & 35.3494 & 106.6694 & 2 & 13 & 3 & 18 & 330 \\
\hline SA39 & Penasco \#3 Spring & $s$ & Sandoval & & 27.0 & 35.5940 & 106.8637 & 2 & 16 & 1 & 29 & 1143 \\
\hline SA40 & Penasco \#4 Spring & s & Sandoval & & 27.0 & 35.5940 & 106.8648 & 2 & 16 & 1 & 29 & 1134 \\
\hline SA41 & Salado Warm Spring & $s$ & Sandoval & & 25.0 & 35.5368 & 106.8467 & 2 & 15 & 1 & 16 & 1112 \\
\hline SA42 & San Ysidro Warm Spring & $s$ & Sandoval & & 24 & 35.5478 & 106.8256 & 2 & 15 & 1 & 10 & 1 \\
\hline SA43 & Swimming Pool Spring & $\mathbf{s}$ & Sandoval & & 24.0 & 35.6015 & 106.8555 & 2 & 16 & 1 & 20 & 4123 \\
\hline SA44 & Jemez/PC-1 at $1712 \mathrm{ft}$ (w/drilling fluid) & $w$ & Sandoval & & & 35.8758 & 106.6617 & & & & & \\
\hline SF1 & Guaje \#3 Well & $w$ & Santa $\mathrm{Fe}$ & 88.4 & 30.5 & 35.9089 & 106.2122 & 2 & 19 & 7 & 4 & 133 \\
\hline SF2 & Los Alamos \#1B Well & $w$ & Santa Fe & 694 & 30.5 & 35.8833 & 106.1564 & 2 & 19 & 7 & 13 & 114 \\
\hline SF3 & Los Alamos \#G6 Well & $w$ & Santa $\mathrm{Fe}$ & 617 & 30.5 & 35.9108 & 106.2353 & 2 & 19 & 7 & 6 & 2 \\
\hline SF4 & Los Alamos $\# 6$ Well & $w$ & Santa Fe & 46.9 & 30 & 35.8772 & 106.1742 & 2 & 19 & 7 & 14 & 312 \\
\hline SF5 & Los Alamos \# & $w$ & Santa $\mathrm{Fe}$ & 617 & 30.0 & 35.9061 & 106.2025 & 2 & 19 & 7 & 4 & \\
\hline SI1 & Torc well & $w$ & Sierra & & 45.5 & 33.2228 & 107.2561 & 3 & 13 & 4 & 33 & 0 \\
\hline $\mathrm{S} 12$ & TorC artesian well & $w$ & Sierra & & 45 & 33.1350 & 107.2533 & 3 & 13 & 4 & 33 & \\
\hline S13 & TorC Blackstone Mineral Bath & $w$ & Sierra & & 45.0 & 33.1350 & 107.2533 & 3 & 13 & 4 & 33 & 41 \\
\hline S14 & TorC well & $w$ & Sierra & & 44.5 & 33.1358 & 107.2553 & 3 & 13 & 4 & 33 & 0 \\
\hline SI5 & TorC Slerra Grande Bath & $w$ & Sierra & & 44 & 33.1350 & 107.2533 & 3 & 13 & 4 & 33 & \\
\hline $\mathrm{SI6}$ & TorC Yucca Lodge well $14 \mathrm{ft}$ & $w$ & Sierra & 4.5 & 43.33 & 33.1350 & 107.2517 & 3 & 13 & 4 & 33 & \\
\hline S17 & TorC Yucca Lodge & $w$ & Sierra & & 43 & 33.1350 & 107.2533 & 3 & 13 & 4 & 33 & \\
\hline $\mathrm{s} 18$ & TorC Yucca Lodge outdoor pool & $w$ & Sierra & & 41.67 & 33.1350 & 107.2517 & 3 & 13 & 4 & 33 & \\
\hline S19 & TorC Sierra Mineral Bath & $w$ & Sierra & & 41.0 & 33.1367 & 107.2483 & 3 & 13 & 4 & 33 & 24 \\
\hline S110 & TorC warm spring & $\mathbf{s}$ & Sierra & & 41.0 & 33.1333 & 107.2417 & 3 & 13 & 4 & 34 & 32 \\
\hline Sl11 & TorC Yucca Lodge & $w$ & Sierra & & 41.0 & 33.1350 & 107.2517 & 3 & 13 & 4 & 33 & 41 \\
\hline SI12 & TorC Old Govemment Spring & $s$ & Sierra & & 40 & 33.1333 & 107.2333 & 3 & 13 & 4 & 34 & 0 \\
\hline SI13 & Torc well & $w$ & Sierra & & 40 & 33.1294 & 107.2572 & 3 & $\overline{13}$ & 4 & 33 & 344 \\
\hline S114 & TorC well & $w$ & Sierra & 30.5 & 40 & 33.1300 & 107.2525 & 3 & 13 & 4 & 33 & 434 \\
\hline S115 & TorC Ponce De Leon Spring & $w$ & Sierra & 75.3 & 40 & 33.1278 & 107.2531 & 3 & 14 & 4 & 4 & 412 \\
\hline SI16 & TorC Geronimo (State) Springs & s & Sierra & & 38.5 & 33.1294 & 107.2550 & 3 & 13 & 4 & 33 & 433 \\
\hline SI17 & Hillsboro Warm Spring & $\mathbf{s}$ & Sierra & & 34.5 & 32.9533 & 107.5817 & 3 & 15 & 7 & 5 & 21 \\
\hline S118 & Victorio Land and Cattle Co. \#1 & $w$ & Sierra & & 34.4 & 33.4100 & 106.7900 & 3 & 10 & 1 & 25 & 1 \\
\hline SI19 & Sun Oil Test Well & $w$ & Sierra & & 34 & 33.4250 & 107.0167 & 3 & 10 & 2 & 25 & 100 \\
\hline S120 & Derry Warm Springs & $s$ & Sierra & & 34 & 32.7917 & 107.2717 & 3 & 17 & 4 & 32 & 213 \\
\hline$S \mid 21$ & well & $w$ & Sierra & & 34 & 32.7953 & 107.2769 & 3 & 17 & 4 & 29 & 343 \\
\hline SI22 & well & $w$ & Sierra & & 31 & 32.6333 & 107.3167 & 3 & 19 & 5 & 25 & $131 \mathrm{~A}$ \\
\hline S123 & warm spring & 5 & Sierra & & & 33.2783 & 107.5633 & 3 & 12 & 7 & 9 & \\
\hline S124 & TorC "warm spring" & s & Sierra & & & 33.1350 & 107.2533 & 3 & 13 & 4 & 33 & \\
\hline $\mathrm{S} 125$ & Bamey lorio \#1 Fee & $w$ & Sierra & 466.3 & & 33.0600 & 107.0300 & 3 & 14 & 5 & 25 & 41 \\
\hline SJ1 & well & $w$ & San Juan & 1738.6 & 62 & 36.0469 & 107.7881 & 1 & 21 & 9 & 16 & 44233 \\
\hline $5 \sqrt{2}$ & well & $w$ & San Juan & 1547.2 & 57 & 36.0536 & 107.7928 & 1 & 21 & 9 & 16 & 23233 \\
\hline S.J3 & Navajo well & $w$ & San Juan & & 51.8 & 36.2578 & 108.3247 & & & & & \\
\hline S. 4 & Dome Well Chaco & $w$ & San Juan & & 42 & 36.0622 & 107.8656 & 1 & 21 & 10 & 11 & 431 \\
\hline SJ5 & ARCOWS- 2 well & $w$ & San Juan & & 39.9 & 36.8658 & 108.5561 & 1 & 31 & 16 & 30 & $4 \overline{442}$ \\
\hline S. 6 & Navajo well & $w$ & San Juan & 932.7 & 35.5 & 36.0922 & 108.2744 & & & & & \\
\hline SJ7 & Navajo $12 T-630$ Well & $w$ & San Juan & & 33 & 36.7153 & 108.6369 & & & & & \\
\hline $5 \sqrt{8}$ & well & $w$ & San Juan & & 32.8 & 36.0311 & 107.9039 & 1 & 21 & 10 & 21 & 3444 \\
\hline SJ9 & Navajo well & $w$ & San Juan & 524.3 & 32 & 36.7889 & 108.6806 & & & & & \\
\hline SJ10 & Navajo 12T-520 Well & $w$ & San Juan & 542.5 & 31 & 36.7764 & 108.6939 & & & & & \\
\hline SJ11 & well & $w$ & San Juan & & 30.5 & 36.3792 & 108.2267 & 1 & 25 & 13 & 28 & 1212 \\
\hline SJ12 & Navajo 12T-629 Well & $w$ & San Juan & & 30.5 & 36.6822 & 108.6572 & 1 & 29 & 17 & 32 & 31 \\
\hline SM1 & Montezuma Hot Spring \#1 & $w$ & San Miguel & & 55.17 & 35.6533 & 105.2900 & 2 & 17 & 13 & 36 & 44 \\
\hline SM2 & Montezuma Hot Spring \#6 & $w$ & San Miguel & & 50.56 & 35.6533 & 105.2900 & 2 & 17 & 13 & 36 & 44 \\
\hline SM3 & Montezuma Hot Spring & $w$ & San Miguel & & 49 & 35.6533 & 105.2900 & 2 & 17 & 15 & 36 & 44 \\
\hline SM4 & Montezuma Hot Spring \#13 & $w$ & San Miguel & & 41.11 & 35.6533 & 105.2900 & 2 & 17 & 13 & 36 & 44 \\
\hline SM5 & Montezuma Hot Spring \#15 & $w$ & San Miguel & & & 35.6533 & 105.2900 & 2 & 17 & 13 & 36 & 44 \\
\hline SM6 & Montezuma Hot Spring \#16 & $w$ & San Miguel & & & 35.6533 & 105.2900 & 2 & 17 & 13 & 36 & 44 \\
\hline SM7 & Montezuma Hot Spring \#18 & $w$ & San Miguel & & & 35.6533 & 105.2900 & 2 & 17 & 13 & 36 & 44 \\
\hline SM8 & Montezuma Hot Spring \#19 & $w$ & San Miguel & & & 35.6533 & 105.2900 & 2 & 17 & 13 & 36 & 44 \\
\hline SM9 & Montezuma Hot Spring \#2 & $w$ & San Miguel & & & 35.6533 & 105.2900 & 2 & 17 & 13 & 36 & 44 \\
\hline
\end{tabular}




\begin{tabular}{|c|c|c|c|c|c|c|c|c|c|c|c|c|}
\hline SITEID & NAME & $W / S$ & COUNTY & $\begin{array}{c}D E P T H \\
\mathbf{m}\end{array}$ & $\begin{array}{c}\text { TEMP } \\
C \\
\end{array}$ & LATITUDE & LONGITUDE & $Q U A D$ & TWN & RNG & $S E C$ & $\overline{Q T R}$ \\
\hline SM10 & Montezuma Hot Spring $\# 20$ & $\bar{w}$ & San Miguel & & & 35.6533 & 105.2900 & 2 & 17 & 13 & 36 & 44 \\
\hline S01 & core hole & $w$ & Socorro & 205.7 & 42.2 & 34.0800 & 106.9500 & 3 & 3 & 1 & 4 & 433 \\
\hline $\mathrm{SO2}$ & warm well & $w$ & Socorro & 66.5 & 36 & 34.1006 & 107.5419 & 3 & 2 & 7 & 27 & 444 \\
\hline $\mathbf{S O 3}$ & Welty Salty Well & $w$ & Socorro & 234.7 & 35 & 33.7997 & 107.6839 & 3 & 6 & 8 & 8 & 432 \\
\hline SO4 & Bosque del Apache Well \#13 & $\mathbf{w}$ & Socorro & & 33 & 33.7900 & 106.8600 & 4 & 6 & 1 & 17 & 213 \\
\hline SO5 & warm well & $w$ & Socorro & 30.5 & 33 & 33.8058 & 106.8761 & 4 & 6 & 1 & 7 & 213 \\
\hline 506 & Blue Canyon Well & $w$ & Socorto & 91.4 & 32.4 & 34.0467 & 106.9508 & 3 & 3 & 1 & 16 & 323 \\
\hline S07 & Soconto Gallery Spring & $\mathbf{s}$ & Socorro & & 32 & 34.0403 & 106.9383 & 3 & 3 & 1 & 22 & 113 \\
\hline .508 & Socorro/Sedilla Gallery Spring & $s$ & Socorto & & 30 & 34.0378 & 106.9386 & 3 & 3 & 1 & 22 & 1131 \\
\hline $\mathbf{5 0 9}$ & well & $w$ & Socorro & & 30.0 & 33.7633 & 107.3500 & 3 & 6 & 5 & 27 & 32 \\
\hline $\mathbf{5 0 1 0}$ & well & $\mathbf{w}$ & Socorro & & 30 & 34.1706 & 106.7522 & 4 & 2 & 2 & 5 & 223 \\
\hline S011 & Monticello Box Warm Spring & $\mathbf{s}$ & Socorro & & 29.0 & 33.3933 & 107.5817 & 3 & 8 & 7 & 31 & 24 \\
\hline S012 & Monticello Box Warm Spring & $\mathbf{s}$ & Socorro & & 28.0 & 33.5733 & 107.6017 & 3 & 8 & 7 & 31 & 41 \\
\hline 5013 & artesian well & $w$ & Socorro & & 26 & 34.5700 & 107.4400 & 1 & 4 & 6 & 14 & 31 \\
\hline 5014 & Field Artesian Well & $w$ & Socorro & & 25 & 34.5300 & 107.4700 & 1 & 4 & 6 & 33 & 34 \\
\hline S015 & Ojo Saladito Spring & $\mathbf{s}$ & Socorro & & 24 & 34.5100 & 107.1300 & 1 & 3 & 3 & 4 & 24 \\
\hline S016 & Cook Spring & $\mathbf{s}$ & Socorro & & & 34.0467 & 106.9367 & 3 & 3 & 1 & 17 & 31 \\
\hline TS1 & Hondo Hot Spring & $\mathbf{s}$ & Taos & & 40.6 & 36.5283 & 105.7150 & 2 & 27 & 12 & 36 & 42 \\
\hline TS2 & Mamby Hot Spring & $\mathbf{s}$ & Taos & & 38 & 36.5222 & 105.7222 & 2 & 26 & 11 & 1 & 120 \\
\hline TS3 & warm spring & $w$ & Taos & & 37 & 36.5308 & 105.7117 & 2 & 27 & 12 & 31 & 311 \\
\hline TS4 & Rancho Del Rio Grande Well & $\mathbf{w}$ & Taos & & 32 & 36.3236 & 105.6056 & & & & & \\
\hline TS5 & Ponce de Leon Hot Spring & s & Taos & & & 36.3233 & 105.6083 & 2 & 24 & 13 & 7 & 333 \\
\hline VA1 & well & $w$ & Valencia & 219.5 & 80 & 34.7800 & 106.6400 & 2 & 6 & 3 & 5 & 234 \\
\hline VA2 & well & $\mathbf{w}$ & Valencia & 801 & 32.5 & 34.8536 & 106.7781 & 2 & 7 & 2 & 7 & \\
\hline
\end{tabular}




\section{APPENDIX 2}

\section{TABLES OF COMPLETE CHEMICAL ANALYSES}

NOTES:

SITE ID

SAMPLE

NAME

TEMP

CHEMISTRY

$\mathrm{mg} / \mathrm{L}$

uS/cm

TDS

TDS (sum)

CHARG BAL \%

COND BAL \%

MASS BAL \% geothermal site number

cited or assigned sample number or designation

(see Appendix 4 for dates and data source

well or spring name

temperature ${ }^{\circ} \mathrm{C}$

units as shown

milligrams per liter

microsiemens per centimeter

analytical total dissolved solids

arithmetic sum dissolved constituents/total dissolved solids charge balance (see Reed and Mariner, 1991)

conductance balance (see Reed and Mariner, 1991) mass balance (see Reed and Mariner, 1991) 


\begin{tabular}{|c|c|c|c|c|c|c|c|c|c|c|c|c|c|c|c|c|c|c|c|c|c|c|c|c|c|c|}
\hline SITE ID & SAMPLE & NAME & $\begin{array}{c}T M P \\
C\end{array}$ & \begin{tabular}{|l|}
$p H$ \\
field \\
\end{tabular} & $\begin{array}{l}\mathrm{pH} \\
\mathrm{lab} \\
\end{array}$ & $\begin{array}{l}\text { COND } \\
\mu \mathrm{S} / \mathrm{cm}\end{array}$ & $\begin{array}{c}\text { TDS } \\
\text { mod } \\
\end{array}$ & $\begin{array}{c}\text { TDS } \\
\text { (sum) }\end{array}$ & $\begin{array}{c}\mathrm{Na} \\
\mathrm{mg} \mathrm{L}\end{array}$ & $\begin{array}{c}K \\
m \neq / L\end{array}$ & $\begin{array}{c}\mathrm{Ca} \\
\mathrm{m} / \mathrm{L}\end{array}$ & $\begin{array}{c}\mathrm{Mg} \\
\mathrm{mg} / \mathrm{L}\end{array}$ & $\begin{array}{c} \\
m q / L\end{array}$ & \begin{tabular}{c|}
$S r$ \\
$m g / h$
\end{tabular} & $\begin{array}{l}\mathrm{HCO} 3 \\
\mathrm{mog} /\end{array}$ & $\begin{array}{l}\text { SO4 } \\
\text { mol }\end{array}$ & $\begin{array}{c}C l \\
m g / 2\end{array}$ & \begin{tabular}{|c|}
$F$ \\
$m \alpha / h$
\end{tabular} & \begin{tabular}{l|}
$B r$ \\
$g / L$
\end{tabular} & \begin{tabular}{|c|}
$B$ \\
$m q L$ \\
\end{tabular} & $\begin{array}{c}\mathrm{Fe} \\
\mathrm{mo} / \mathrm{L}\end{array}$ & $\begin{array}{l}\mathrm{NO3} \\
\mathrm{mol} /\end{array}$ & $\begin{array}{r}\text { SIO2 } \\
\alpha^{\prime} L \\
\end{array}$ & $\begin{array}{l}\text { CHRG } \\
\text { BAL \& }\end{array}$ & \begin{tabular}{|l|} 
COND \\
BAL $\%$ \\
\end{tabular} & $\begin{array}{l}\text { MASS } \\
\text { BAL \% }\end{array}$ \\
\hline BE10 & 209 & well & 30.0 & 8.9 & & 524 & 340 & 430 & 110 & 2 & 6.1 & 0.4 & & & 165 & 84 & 13 & 1.1 & & 0.26 & 0.03 & 7.97 & 40 & 2.94 & 1.27 & 26.43 \\
\hline CA1 & GILA7 & hot spring & 64.8 & & 7.92 & & 548 & 620 & 151.5 & 3.5 & 15.4 & 0.1 & 0.43 & 0.03 & 131.1 & 118 & 104.3 & 9.5 & & 0.07 & & & 85.9 & 3.40 & & 13.11 \\
\hline CA3 & LFHS12-74 & Lower Frisco Hot Spring & \begin{tabular}{|l|}
35.2 \\
\end{tabular} & 6.5 & & 1200 & & 855 & 236 & 7.5 & 11 & 7.8 & \begin{tabular}{|l|}
0.41 \\
\end{tabular} & & 167 & 40 & 327 & 1.5 & 0.61 & & & & 56 & 4.77 & 2.44 & \\
\hline CA3 & M77-СТ2 & Lower Frisco Hot Spring & 37.0 & 7.35 & & & 1015 & 1013 & 270 & 15 & 46 & 6.2 & 0.43 & & 121 & 39 & 430 & 1.4 & & 0.26 & & & 84 & 0.01 & & 0.17 \\
\hline $\mathrm{CA} 3$ & SNM-19 & Lower Frisco Hot Spring & \begin{tabular}{|l|}
50.5 \\
\end{tabular} & 6.80 & 7.64 & 1894 & 1142.9 & 1144 & 307 & 19 & 49.4 & 5.7 & 0.53 & 0.37 & 131 & 43 & 499 & 0.51 & & 0.23 & & & 88 & 0.84 & \begin{tabular}{|l|}
10.99 \\
\end{tabular} & 0.07 \\
\hline CA4 & $\sqrt{3}$ & Lower Frisco Hot Spring & \begin{tabular}{|l|}
43.3 \\
\end{tabular} & & 7.89 & & 992 & 1090 & 307.2 & 15.6 & 49.7 & 6.8 & 0.48 & 0.33 & 129.4 & 57.6 & 445.3 & 1.43 & 0.56 & 0.28 & & & 75.2 & 2.78 & & 9.86 \\
\hline CA4 & $\sqrt{5}$ & Lower Frisco Hot Spring & \begin{tabular}{|l|}
48.9 \\
\end{tabular} & & 7.79 & & 1280 & 1353 & 406 & 18.8 & 54.3 & 6.9 & 0.65 & \begin{tabular}{|l|}
0.43 \\
\end{tabular} & 107.4 & 90.3 & 574.3 & 1.8 & 0.56 & 0.38 & & & 90.9 & 3.81 & & 5.68 \\
\hline CA4 & SNM-18 & Lower Frisco Hot Spring & 45.0 & 6.80 & 7.64 & 1495 & \begin{tabular}{|l|}
925.8 \\
\end{tabular} & 924 & 232 & 10.8 & 46.3 & 7.2 & \begin{tabular}{|l|}
0.4 \\
\end{tabular} & 0.35 & 138 & 35.6 & 372 & 1.5 & & 0.13 & & & 80 & 0.89 & 10.79 & 0.16 \\
\hline CA6 & $\mathrm{J4}$ & Lower Frisco Hot Spring & 40.0 & & 7.95 & & 768 & 817 & 215.6 & 11.3 & 39.3 & 7.4 & 0.34 & 0.28 & 136.7 & 44.2 & 294.6 & 1.49 & 0.43 & 0.22 & & & 64.8 & 3.09 & & 6.34 \\
\hline CA7 & $\sqrt{2}$ & Upper Frisco Hot Spring & \begin{tabular}{|l|}
36.7 \\
\end{tabular} & & 9.60 & & 156 & 202 & 62.8 & 0.4 & 1.2 & & 0.01 & & 72 & 19.2 & 0.4 & 0.72 & 0.31 & 0.03 & & & 45.4 & 26.38 & & 29.76 \\
\hline CA7 & SNM-20 & Upper Frisco Hot Spring & 37.0 & 8.80 & 9.67 & 261 & 211.6 & 135 & 65.1 & 0.4 & 1.3 & 0.04 & & & 14.6 & 1.5 & $\frac{\pi+4}{2}$ & 0.75 & & & & & 49 & 77.63 & 11.50 & 36.35 \\
\hline CAB & MFG3 & Gila Middle Fork Hot Spring & 36.0 & & 8.09 & & 192 & 297 & 43.7 & 1.2 & 16.8 & 9.6 & & & 139.7 & 28.3 & 3.9 & 5.28 & & 0.07 & & & 56.5 & 5.92 & & 54.71 \\
\hline \begin{tabular}{|l|} 
CA10 \\
\end{tabular} & DL-32-NM & Pueblo Windmill & 33.8 & 6.00 & & & & 1270 & 140 & 13 & 130 & 29 & & & 561 & 310 & 68 & 3.4 & & 0.36 & 1.6 & & 14 & 7.42 & & \\
\hline \begin{tabular}{|l|} 
CA13 \\
\end{tabular} & GS54856 & test well & 32.2 & & 8.70 & 2710 & 2540 & 2430 & 224 & 1.6 & 505 & & & & 15 & 1604 & 52 & 7.8 & & 0.39 & & & 20 & 0.76 & 29.09 & $\overline{4.34}$ \\
\hline \begin{tabular}{|l|} 
CA14 \\
\end{tabular} & 130 & well & \begin{tabular}{|l|}
32.0 \\
\end{tabular} & 9 & & 330 & 256 & 283 & 65 & 2.7 & 0.9 & 0.1 & & & 120 & 23 & 13 & 1.4 & & \begin{tabular}{|l|}
0.1 \\
0.1
\end{tabular} & 0.05 & 3.99 & 53 & 1.09 & 10.62 & 10.64 \\
\hline CA15 & 119 & well & \begin{tabular}{|l|}
32.0 \\
\end{tabular} & 8.7 & & 2710 & 2540 & 2417 & 220 & 1.6 & 500 & & & & 15 & 1600 & 52 & 7.8 & & 0.39 & 0.03 & 0.2 & 20 & 1.25 & 27.53 & 4.84 \\
\hline CA16 & MFG1 & Gila Middle Fork Hot Spring & 31.0 & & 8.08 & & 196 & 299 & 40 & 1.6 & 20.4 & 2.6 & & & 145.8 & 29.8 & 3.2 & 4.86 & & 0.05 & & & 51 & 5.39 & & 52.71 \\
\hline \begin{tabular}{|l|} 
CA18 \\
\end{tabular} & MEAD.10 & Gila Mlddle Fork Meadows HS & \begin{tabular}{|l|}
27.5 \\
\end{tabular} & & 7.60 & 215 & 150 & 167 & 25 & 2 & 17 & 1.8 & & & 94 & 11 & 10 & 3.4 & & & & & 2.8 & 2.19 & 0.70 & 11.33 \\
\hline \begin{tabular}{|l|} 
CA18 \\
\end{tabular} & MEAD.12 & Glla MIddle Fork Meadows HS & 29.5 & & 7.10 & 220 & 150 & 171 & 25 & 1.8 & 17 & 1.8 & & & 82 & 11 & 12 & 3.4 & & & & & 17 & 0.93 & 3.19 & 14.00 \\
\hline CA18 & MEAD-14 & Gila Middle Fork Meadows HS & 32.5 & & 7.60 & 215 & 145 & 174 & 25 & 1.7 & 19 & 1.8 & & & 92 & 90 & 6 & 3.4 & & & & & 15 & 3.79 & 3.59 & 19.93 \\
\hline \begin{tabular}{|l|} 
CA18 \\
\end{tabular} & MEAD-19 & Glla Middle Fork Meadows HS & 27.5 & & 7.70 & 215 & 155 & 171 & 27 & 1.8 & 18 & 1.7 & & & 88 & 5.5 & 6 & 3.4 & & & & & 20 & 8.49 & 5.05 & 10.58 \\
\hline \begin{tabular}{|l|} 
CA18 \\
\end{tabular} & MEAD-5 & Gila Middle Fork Meadows HS & 28.0 & & 7.50 & 220 & 160 & 173 & 24 & 1.8 & 17. & 1.8 & & & 90 & 5.5 & 8 & 3.4 & & & & & 21 & 2.26 & 5.16 & 7.81 \\
\hline $\begin{array}{ll}\text { CA2O } \\
\end{array}$ & US107 & Zuni Salt Lake Warm Spring & 26.0 & & 8.24 & & 220152 & 202370 & 66205 & 1173 & 195.2 & 4885 & & & 238 & 28722 & 100938 & 0.73 & & 3 & & & 10 & 1.89 & & 8.08 \\
\hline CA21 & GS62058 & Gila Middle Fork Pool HS & & & 7.90 & 771 & 508 & 607 & 177 & 4.1 & 16 & 0.1 & 0.2 & 0.2 & 128 & 84 & 108 & 9.6 & & 0.12 & & & 80 & 7.76 & 12.12 & 19.55 \\
\hline CA21 & M77-CT1 & Gila Middle Fork Pool HS & 66.0 & 7.78 & & & 587 & 581 & 150 & 3.1 & 14 & 0.1 & 0.36 & & 135 & 79 & 105 & 9.5 & & 0.11 & & & 85 & 0.30 & & 0.99 \\
\hline CA21 & PHS-SUM & Gila Middle Fork Pool HS & 65.3 & & 8.20 & 720 & 514 & 479 & 146 & 3.9 & 16 & 0.1 & 0.4 & & 94 & 20 & 107 & 3.4 & 0.25 & 0.1 & & & 88 & 17.30 & 1.60 & 6.78 \\
\hline CA21 & SNM-14 & Gila Middle Fork Pool HS & 65.5 & 6.80 & 7.90 & 777 & 580.8 & 580 & 145 & 3 & 16.6 & 0.13 & 0.38 & 0.23 & 139 & 79.9 & 108 & 9.4 & & 0.05 & & & 78 & 1.35 & 6.26 & 0.19 \\
\hline CA22 & MFG2 & Gila Middle Fork Hot Spring & 37.0 & & 8.07 & & 188 & 289 & 41.8 & 0.8 & 19.2 & 1.6 & & & 128.1 & 31.7 & 4.2 & \begin{tabular}{|l|}
5.28 \\
\end{tabular} & & 0.02 & & & 56 & 3.74 & & 53.56 \\
\hline CA23 & MFG4 & Gilla Middle Fork Hot Spring & \begin{tabular}{|l|}
26.0 \\
\end{tabular} & & 8.15 & & 168 & 267 & 37.5 & 0.8 & 14.8 & 1.5 & & & 931.2 & 19.2 & 3.2 & 5.07 & & & & & 54 & 7.26 & & 59.09 \\
\hline CA23 & SW307 & Gila Middle Fork Hot Spring & 32.0 & & 8.44 & & 224 & 227 & 34 & 0.8 & 14 & 1.3 & & & 97.6 & 23.2 & 1.1 & \begin{tabular}{|l|}
5.01 \\
\end{tabular} & & & & & 50 & 1.55 & & 1.34 \\
\hline CA23 & SW306 & Gila Middle Fork Hot Spring & 34.0 & & 8.12 & & 192 & 233 & 35.2 & 0.8 & 14.2 & 1.3 & & & 82 & 44 & 1.4 & 5.01 & & & & & 49.5 & 3.98 & & 21.57 \\
\hline $\mathrm{CB} 2$ & 210 & well & 52.8 & 6.3 & 7.2 & 7000 & 5450 & 6652 & 1100 & 47 & 730 & 110 & 4 & 8.6 & 1320 & 2000 & 1300 & 0.6 & 3 & 3.2 & 2 & & 24 & 2.41 & 36.14 & 22.06 \\
\hline $\mathrm{CB} 6$ & 178 & well & 36.0 & & & 989 & & 797 & 42 & 7 & 120 & 36 & 0.05 & & 257 & 300 & 19 & 0.7 & & 0.03 & & & 15 & 0.31 & \begin{tabular}{|l|}
10.84 \\
\end{tabular} & \\
\hline CB9 & 193 & well & 34.0 & 6.8 & & 25200 & 18500 & 19156 & 5600 & 55 & 460 & 460 & 0.81 & & 1360 & 4300 & 6900 & 1.6 & & & 3.4 & 0.6 & 15 & 0.10 & 21.40 & 3.55 \\
\hline CB9 & 197 & well & 31.5 & 6.7 & & 18000 & & 14600 & 4100 & 59 & 320 & 330 & & & 1770 & 4300 & 3700 & 2.9 & & 3.5 & & 0.3 & 14 & 0.02 & \begin{tabular}{|l|}
23.87 \\
\end{tabular} & \\
\hline CB13 & LUC-11 & Salado Spring & 25.0 & 6.46 & & 3800 & & 3583 & 195 & 21 & 670 & \begin{tabular}{|l|}
92.4 \\
\end{tabular} & 0.52 & \begin{tabular}{|l|}
9.42 \\
\end{tabular} & 425 & 1990 & 156 & 1.87 & & 0.68 & 5.83 & & 15 & 2.47 & \begin{tabular}{|l|}
32.48 \\
\end{tabular} & \\
\hline DA1 & \begin{tabular}{|l|} 
CHAF55-25 \\
\end{tabular} & Chaffee 55.25 & 69.0 & & 7.46 & 2300 & 1480 & 1642 & 350.8 & 53.2 & \begin{tabular}{|l|}
96.9 \\
\end{tabular} & 33 & & & 373.4 & 198 & 482.2 & 2.41 & & & \begin{tabular}{|l|}
0.1 \\
\end{tabular} & & 51.5 & 0.41 & 5.09 & 10.91 \\
\hline DA2 & SNM-5 & Radium Hol Springs Bailey Well \#15 & 70.0 & 6.00 & 7.11 & 7050 & 3944.3 & 3942 & 1140 & 164 & 131 & 11.2 & 1.26 & \begin{tabular}{|l|}
2.33 \\
\end{tabular} & 438 & 253 & 1720 & 5.44 & & 0.82 & & & 75 & 0.18 & 12.80 & 0.06 \\
\hline DA4 & \begin{tabular}{|l|} 
CHF35-25 \\
\end{tabular} & Chaffee 35-25 & 68.0 & & 8.05 & 2580 & 1626 & 1871 & 397.5 & 54.7 & 129.2 & 31.2 & & & 394.2 & 300 & 496.3 & 2.16 & & 0.36 & 0.22 & 8.37 & 56.5 & 1.62 & 7.38 & 15.05 \\
\hline DA7 & \begin{tabular}{|l|} 
CHF $12-24$ \\
\end{tabular} & Chaffee 12.24 & 65.0 & & 7.57 & 3000 & 1968 & 1809 & 392.4 & 58.3 & 107.4 & 28 & & & 448.7 & 220.8 & 499.2 & 2.2 & & 0.18 & 0.13 & 0.31 & 50.9 & 0.14 & 12.59 & 8.10 \\
\hline DA9 & S76BAILEY & Radium Hot Springs Bailey's bathhouse & 60.8 & & 7.96 & 5600 & 3731 & 4615 & 1212 & 182 & 142 & 11.7 & 1.18 & & 350 & 232 & 2400 & 3.61 & 2.35 & 0.82 & & & 77.5 & 8.95 & 17.13 & 23.70 \\
\hline DA12 & $\mathrm{B2}$ & Radium Hot Springs Hotel Well \#2 & 53.0 & & 8.16 & & 3532 & 3752 & 9135.9 & 167 & 118.6 & 15.2 & 1.18 & \begin{tabular}{|l|l|}
2.49 \\
\end{tabular} & 378.3 & 263.2 & 1593.6 & 4.44 & 1.54 & 0.86 & & & 69.9 & 3.55 & & 6.23 \\
\hline DA12 & M77-DA1 & Radium Hot Springs Hotel Well \#2 & 52.0 & 7.13 & & & 3857 & 3804 & 1100 & 160 & 120 & 15 & 1.2 & & 414 & 260 & 1650 & 4.8 & & 0.68 & & & 78 & 0.29 & & 1.38 \\
\hline DA12 & \begin{tabular}{|l|} 
RHSHOT2 \\
\end{tabular} & Radium Hot Springs Hotel Well \#2 & 60.0 & & 8.33 & 5800 & & 4253 & 1373 & 164 & 65 & 11 & 1.01 & & 253 & 208 & 2100 & \begin{tabular}{|l|}
3.02 \\
\end{tabular} & 2.5 & 1.06 & & & 71 & 0.22 & 17.61 & \\
\hline DA12 & \begin{tabular}{|l|} 
S76HRSW2 \\
\end{tabular} & Radium Hot Springs Hotel Well \#2 & 60.0 & 6.70 & & & & 3930 & 1120 & 150 & 127 & 13.5 & 1.5 & & 480 & 300 & 1680 & 5.1 & 0.1 & 0.9 & & & 52 & 1.28 & & \\
\hline DA12 & SNM-6 & Radium Hot Springs Hotel Well \#2 & 43.0 & 7.00 & 7.41 & 6530 & 3728.4 & 3727 & 1070 & 146 & 136 & 13 & 1.27 & \begin{tabular}{|l|l|}
2.33 \\
\end{tabular} & 438 & 263 & 1580 & 4.73 & & 0.77 & & & 72 & \begin{tabular}{|l|}
0.77 \\
\end{tabular} & 10.61 & 0.03 \\
\hline DA13 & $280 L C 1$ & LC-1 & 67.9 & & 8.29 & 922 & 576 & 730 & 214.5 & 6.2 & 10.2 & $\frac{\pi}{1}$ & & & 219.7 & 149.8 & 102.1 & 1.9 & & 0.45 & & 0.09 & 24.3 & \begin{tabular}{|l|}
1.92 \\
\end{tabular} & 9.33 & 26.78 \\
\hline DA14 & SNM-7 & Radium Hot Springs Roy Smith Well & 50.0 & 6.70 & 7.01 & 5230 & 2978.5 & 2973 & 842 & 114 & 120 & 12.9 & 0.98 & 2 & 348 & 250 & 1220 & 3.97 & & 0.6 & & & 59 & 1.35 & 10.56 & 0.17 \\
\hline DA15 & NMSUB1479 & NMSUPG-2 & & & 8.35 & 2480 & 1704 & 1819 & 381.6 & 175 & 19.2 & 27 & & & 508.9 & 226.2 & 421.9 & 1.31 & & 0.23 & 0.55 & & 57.5 & 1.57 & 2.22 & 6.76 \\
\hline DA15 & SNM-9 & NMSUPG-2 & 43.0 & 6.50 & 7.49 & 3290 & 2232.6 & 2215 & 447 & 47 & 145 & 38.5 & 0.49 & 3.96 & 734 & 135 & 590 & 0.82 & & 0.3 & & & 73 & 0.52 & 5.14 & 0.78 \\
\hline DA17 & $\begin{array}{ll}\text { LASALTNA } \\
\end{array}$ & Nations Well & 45.1 & & 8.07 & 2400 & 1983 & 2340 & 488 & 55 & 142 & 32 & 0.46 & & 348 & 223 & 980 & 1.62 & 1.61 & 0.6 & & & 68 & 8.05 & 35.08 & 18.02 \\
\hline \begin{tabular}{l|l} 
DA17 \\
\end{tabular} & NATIONW81 & Nations Well & 43.0 & & 7.60 & 3040 & & 2311 & 450 & 46 & 190 & 34 & & & 700 & 250 & 580 & 1.4 & & & 2 & 0.01 & 58 & 0.13 & 8.65 & \\
\hline DA18 & 47 & well & 43.0 & \begin{tabular}{|l|}
7.6 \\
\end{tabular} & & 3040 & & 2309 & 450 & 46 & 190 & 34 & & & 700 & 250 & 580 & 1.4 & & & 0.01 & \begin{tabular}{|l|}
0.04 \\
\end{tabular} & 58 & 0.13 & 8.65 & \\
\hline
\end{tabular}




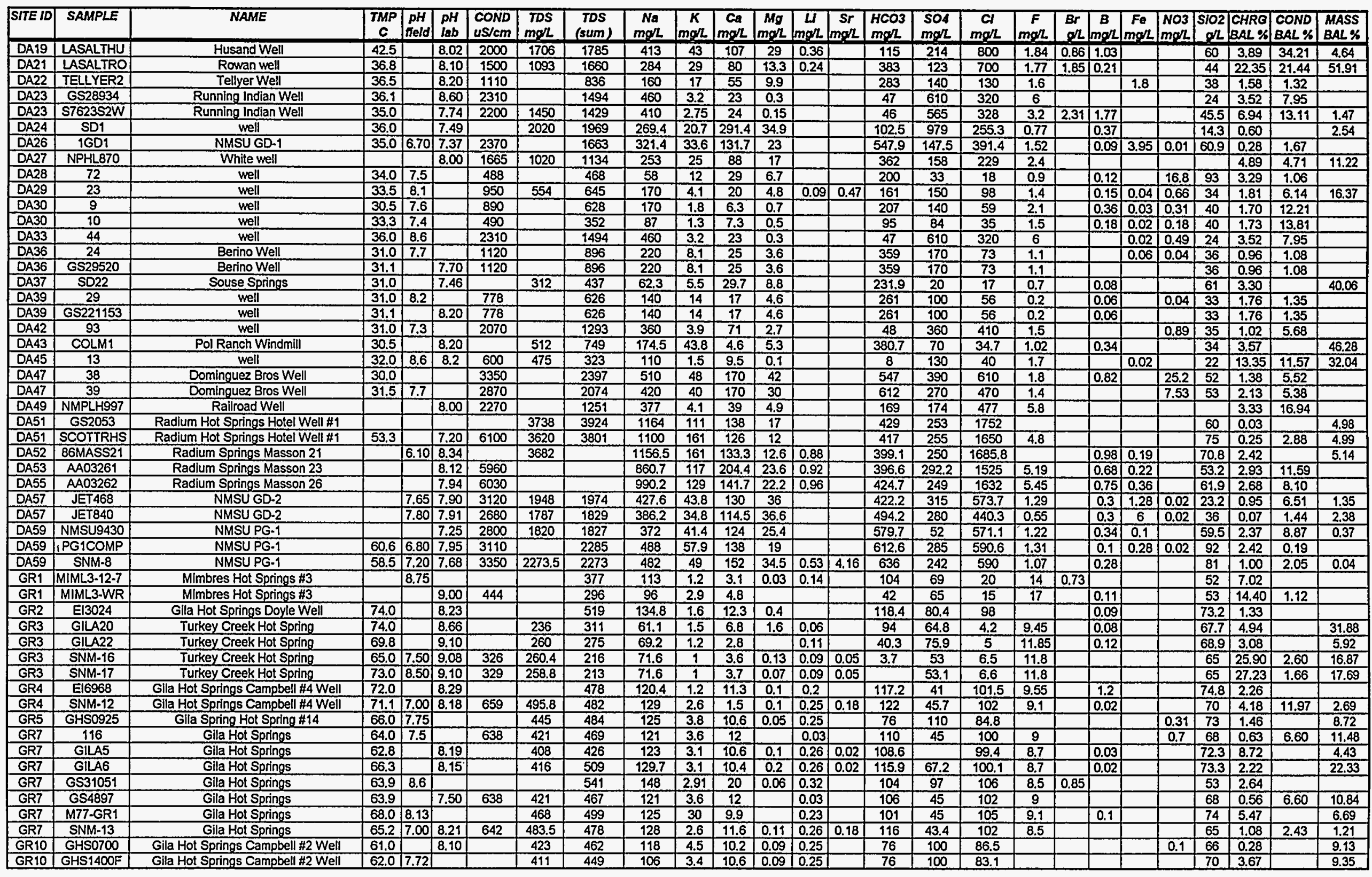




\begin{tabular}{|c|c|c|c|c|c|c|c|c|c|c|c|c|c|c|c|c|c|c|c|c|c|c|c|c|c|c|}
\hline SITE ID & SAMPLE & NAME & \begin{tabular}{|c|} 
TMPP \\
$C$ \\
\end{tabular} & $\begin{array}{l}p H \\
\text { feld }\end{array}$ & \begin{tabular}{|c|}
$p H$ \\
$l a b$ \\
\end{tabular} & $\begin{array}{l}\text { COND } \\
\mathrm{uS} / \mathrm{cm} \\
\end{array}$ & \begin{tabular}{|c|} 
TDS \\
mogh \\
\end{tabular} & $\begin{array}{c}\text { TDS } \\
\text { (sum) }\end{array}$ & $\begin{array}{c}\mathrm{Na} \\
\mathrm{mg} / \mathrm{L} \\
\end{array}$ & $\begin{array}{c}K \\
m g / L \\
\end{array}$ & $\begin{array}{c}\mathrm{Ca} \\
\mathrm{mal} \\
\end{array}$ & \begin{tabular}{|c|}
$\mathrm{Mg}$ \\
$\mathrm{mg} / \mathrm{L}$
\end{tabular} & \begin{tabular}{|c|} 
\\
$m g / L$ \\
\end{tabular} & \begin{tabular}{|c|}
$\mathbf{S r}$ \\
$\mathrm{mg} / \mathrm{L}$ \\
\end{tabular} & $\begin{array}{l}\mathrm{HCO} 3 \\
\mathrm{mo/L}\end{array}$ & $\begin{array}{c}\text { SO4 } \\
\text { mol }\end{array}$ & $\begin{array}{c}c l \\
m o / 2\end{array}$ & \begin{tabular}{c|}
$F$ \\
$m g h$ \\
\end{tabular} & $\begin{array}{l}B r \\
g l\end{array}$ & \begin{tabular}{|c|} 
B \\
$m^{\prime} / L$
\end{tabular} & \begin{tabular}{|c|}
$F_{\theta}$ \\
$g / L$
\end{tabular} & \begin{tabular}{ll|}
$\mathrm{NO3}$ \\
$\mathrm{mg} / \mathrm{L}$
\end{tabular} & $\begin{array}{r}5102 \\
g / L\end{array} \mid$ & $\begin{array}{l}\text { CHRo } \\
\text { BAL \% }\end{array}$ & $\begin{array}{l}\text { COND } \\
\text { BAL \% }\end{array}$ & $\begin{array}{l}\text { MASS } \\
\text { BAL \% }\end{array}$ \\
\hline \begin{tabular}{l|l} 
GR10 \\
\end{tabular} & GHS1400F & Gila Hot Springs Campbell \#2 Well & 62.0 & & & & 411 & 449 & 106 & & & & & & & 100 & & & & & & & & & & \\
\hline \begin{tabular}{|l|} 
GR10 \\
\end{tabular} & GHS1445 & Gila Hot Springs Campbell \#2 Well & 61.0 & 7.97 & & & 418 & 457 & 120 & \begin{tabular}{|l|}
3.4 \\
3.4
\end{tabular} & & \begin{tabular}{|l|l|}
0.08 \\
\end{tabular} & $\mid \begin{array}{l}\mid-25 \\
0.25 \\
\end{array}$ & & $\frac{70}{76}$ & $\frac{100}{100}$ & $\frac{0.1}{86.5}$ & & & & & & $\frac{10}{60}$ & .95 & & \\
\hline GR10 & \begin{tabular}{|l} 
GHS1610 \\
\end{tabular} & Gila Hot Springs Campbell \#2 Well & 62.0 & 7.97 & & & $\frac{416}{415}$ & 454 & $\frac{120}{118}$ & 3.4 & 10.0 & \begin{tabular}{|l|l|}
0.08 \\
\end{tabular} & \begin{tabular}{|c|}
0.25 \\
0.25 \\
\end{tabular} & & $\frac{70}{76}$ & $\frac{100}{100}$ & 80.5 & & & & & & & 30 & & \\
\hline GR10 & GHS1610 & Gila Hot Springs Campbell \#2 Well & 62.0 & 7.97 & & & $\frac{485}{415}$ & 455 & $\frac{110}{118}$ & 3.4 & 10.28 & 0.08 & \begin{tabular}{|l|}
.25 \\
0.25 \\
\end{tabular} & & $\frac{10}{76}$ & $\frac{100}{100}$ & $\frac{81.4}{81.8}$ & & & & & & $\frac{5}{65}$ & $\frac{30}{19}$ & & \\
\hline \begin{tabular}{|l|} 
GR10 \\
CD99 \\
\end{tabular} & GHS2252: & Gila Hot Springs Campbell \#2 Well & 60.0 & & 7.70 & & 428 & 467 & 120 & 3.9 & 11 & 0.09 & 0.25 & & $\frac{76}{76}$ & $\underline{n}$ & 84. & & & & & & & & & \\
\hline \begin{tabular}{|l|l|} 
GR13 \\
\end{tabular} & GHS0935 & Gila Spring Hot Spring \#41 & 58.0 & & & & 426 & 465 & 121 & 3.6 & $\frac{10.2}{10.2}$ & $\frac{08}{0.8}$ & \begin{tabular}{|l|}
0.25 \\
0.25 \\
\end{tabular} & & 76 & $\frac{05}{03}$ & 83.1 & & & & & & 7 & & & \\
\hline GR14 & 86 & Mimbres Hot Springs & 60.5 & 8.3 & & 455 & & 363 & 94 & 1.3 & 3.3 & 0.6 & 0.1 & & 107 & w & $\frac{1.17}{17}$ & 18 & & & & \begin{tabular}{|l|l|l|l|}
0.97 \\
\end{tabular} & & 央 & 4.38 & \\
\hline \begin{tabular}{|l|l|} 
GR14 \\
\end{tabular} & GILA4 & Mimbres Hot Springs & 58.2 & & 8.97 & & 320 & 333 & 91.7 & 1.2 & $\frac{3.5}{2.4}$ & 0.0 & $\mid 0.11$ & & $\frac{101}{67.1}$ & $\frac{85}{84}$ & $\frac{11}{14.5}$ & 10 & & & & 0.91 & 55.6 & - & 4.30 & \\
\hline GR16 & MIM-L12 & Mimbres Hot Springs $\# 12$ & 56.4 & 8.24 & & & & 286 & 115 & 1.9 & 3 & & \begin{tabular}{|l|}
0.13 \\
\end{tabular} & & 89 & 9 & & & 0.2 & 12 & & & & 17 & & \\
\hline GR19 & 73 & Faywood Hot Springs & 53.0 & 7.4 & & 605 & 384 & 534 & 85 & 7.8 & 38 & 7.3 & & & 278 & $\frac{5}{52}$ & $\frac{20}{16}$ & $\frac{2.2}{6.8}$ & & & & 0.2 & $\overline{43}$ & $\frac{11}{3}$ & & \\
\hline \begin{tabular}{l|l|} 
GR19 \\
\end{tabular} & 74 & Faywood Hot Springs & 53.5 & 7.1 & & 603 & & 518 & 79 & 7.6 & 34 & 7.4 & 0.13 & & 277 & 49 & 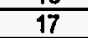 & 5.9 & & & & 0.48 & Tu & 22 & 25 & \\
\hline GR19 & FAY S76-1 & Faywood Hot Springs & & 6.95 & & & & 581 & 100 & 7.1 & 36 & 9.1 & 0.19 & & 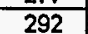 & 76 & & 品 & & & & & $\frac{41}{39}$ & & & \\
\hline \begin{tabular}{|l|l|} 
GR19 \\
\end{tabular} & FAY-WRD & Faywood Hot Springs & & & 8.40 & 504 & 330 & 443 & 87 & $\frac{11}{11}$ & $\frac{14}{14}$ & $\frac{6.3}{6.3}>25$ & \begin{tabular}{|c|c|c|}
0.07 \\
\end{tabular} & & 197 & $\frac{25}{55}+2>0$ & $\frac{10}{19}$ & 63 & & 261 & & & $\frac{5}{\Delta 7}$ & at & 501 & \\
\hline GR1 & GILA2 & Faywood Hot Springs & 53.8 & & 7.74 & & 492 & 564 & 90.8 & 8.2 & 35.6 & 7.6 & \begin{tabular}{|l|}
0.16 \\
\end{tabular} & 0.1 & 283 & 72 & $\frac{15}{15 .}$ & 0.3 & & & & & \begin{tabular}{|l|}
45.2 \\
45 \\
\end{tabular} & 23 & 0.01 & \\
\hline & GS15112 & Faywood Hot Springs & & 7.10 & & 603 & & 518 & 79 & 7.6 & \begin{tabular}{|l|}
34 \\
\end{tabular} & 7.4 & \begin{tabular}{|l|}
0.13 \\
\end{tabular} & & 20 & 49 & $\frac{17}{17}$ & $\frac{0.1}{5.9}$ & 0.1 & $\mid$\begin{tabular}{|l|}
0.07 \\
0.07
\end{tabular} & & & \begin{tabular}{|l|}
41.4 \\
41
\end{tabular} & $\frac{2.00}{3.22}$ & & \\
\hline GR19 & GS1856 & Faywood Hot Springs & 53.3 & & 7.40 & 605 & 384 & 534 & 85 & 7.8 & 38 & 7.3 & & & & 90 & & 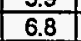 & & & & & 43 & 0.43 & $\frac{1.20}{5.68}$ & \\
\hline 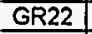 & GILA8 & Gila River Waterfall HS & 43.6 & & 8.08 & & 516 & 593 & 141.9 & 2.7 & 18.4 & 0.8 & 31 & U: & & 33.6 & $\frac{10}{15}$ & 8.7 & & 0.11 & & & 85.9 & 2.99 & & \\
\hline 222 & M77-GR2 & Gila River Waterfall HS & 43.0 & 7.88 & & & 558 & 563 & 145 & 2.7 & 16 & 0.7 & 0.25 & & & 67 & & 8.9 & & 0.14 & & & 72 & 0.76 & & \\
\hline GR23 & FGR-NM & Gila East Fork no name spring & 41.4 & & 8.1 & 581 & 369 & 431 & 116 & 1.5 & \begin{tabular}{|l|}
9.7 \\
\end{tabular} & 0.6 & & & & $\frac{51}{31}$ & & 6.6 & & & & & 64 & 0.87 & 3.31 & \\
\hline & M77-GR3 & Gilla East Fork! & 36.0 & 8.27 & & & 385 & 388 & & & & 0.6 & \begin{tabular}{|l|}
0.17 \\
\end{tabular} & & & $\frac{11}{27}$ & & & & $\mid 0.12$ & & & & & & \\
\hline$G$ & $\overline{L D 2}$ & Muir Ranch well & 33.0 & & 7.86 & & 816 & 893 & & $\frac{11.7}{11.7}$ & 28 & \begin{tabular}{|l|}
0.0 \\
2.7 \\
\end{tabular} & \begin{tabular}{|l|}
0.31 \\
\end{tabular} & 0.15 & 314.8 & $\frac{21}{223,8}$ & $\frac{52}{47.5}$ & $\frac{0.1}{6.9}$ & & & & & $\frac{04}{396}$ & 72 & & \\
\hline & GILA23 & Riverside Well & 29.0 & & \begin{tabular}{l|l}
8.53 &
\end{tabular} & & 292 & 324 & 77.9 & 1.5 & 10.4 & 0.6 & & & 75.7 & 99.4 & $\frac{76.2}{25.9}$ & $\frac{0.5}{105}$ & & $\frac{70}{92}$ & & & 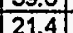 & $\frac{0.12}{673}$ & & \\
\hline GR39 & GILA24 & Spring Canyon Warm Spring & 31.0 & & 8.13 & & 332 & 444 & 92.4 & 1.5 & 18.4 & 1.3 & & & & & 6. & & & & & & 4 & $\frac{104}{44}$ & & \\
\hline GR41 & GILA29 & Mangas Springs & 27.0 & & 8.00 & & 544 & 649 & 34.9 & 7.8 & \begin{tabular}{|l|l|}
87 \\
\end{tabular} & $\frac{1.5}{16.5}$ & \begin{tabular}{|l|}
0.020 \\
0.02
\end{tabular} & & & $\frac{45}{35}$ & $\frac{0.4}{18.8}$ & & & & & & $\left|\frac{3.5}{57.8}\right|$ & $\frac{2.44}{176}$ & & \\
\hline GR42 & $\mathrm{J1}$ & Allen Spring & 25.6 & & 8.12 & & 492 & 582 & 8.3 & 1.6 & 77.8 & 38.2 & & 12 & 89.3 & 48 & $\frac{10.0}{2.5}$ & $\frac{.04}{.05}$ & & & & & $\mid$\begin{tabular}{|l|}
1.8 .5 \\
14.5
\end{tabular} & $\frac{1.10}{0.54}$ & & \\
\hline \begin{tabular}{|lll} 
GR43 & \\
\end{tabular} & & Cliff Warm Spring & 25.0 & & 7.89 & & 164 & 237 & 27.4 & 2.7 & \begin{tabular}{|l|l|}
13 \\
\end{tabular} & 6.9 & $\sqrt{0.03}$ & & $\frac{140.3}{140}>>2$ & $\frac{75}{15 .}$ & 1. & & & & & & $\frac{14.5}{29.3}$ & $\frac{0.12}{412}$ & & \\
\hline \begin{tabular}{|l|} 
HD1 \\
\end{tabular} & SNM-11 & Lightning Dock Burgett Well \#1 & 104.0 & 7.80 & 8.45 & 366 & 1180.5 & 1122 & 324 & 30 & 20 & 0.18 & 0.69 & 0.05 & & 510 & 90. & 11.6 & 0.2 & & & & $\frac{5.04}{145}$ & $\frac{7.24}{6.54}$ & 5.65 & 89 \\
\hline HD2 & & Lightning Dock & & & 9.10 & 1560 & & 106 & 302 & 16 & 18 & 0.1 & 0.66 & & 3 & 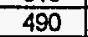 & 8 & 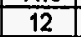 & & 0.45 & & & 103 & 2.39 & 6 & \\
\hline HD2 & S76LD-12 & Llghtning Dock & & & 8.75 & 500 & 1057 & $\overline{1136}$ & 348 & 24 & 16 & 0.1 & \begin{tabular}{|l|}
0.8 \\
\end{tabular} & & 9 & 46 & & 3.7 & & 0.34 & & & 92 & 8.78 & 11.16 & \\
\hline HD4 & 36 & Llghtning Dock & 95.5 & 9.1 & & 60 & & 1058 & 300 & 16 & 18 & 0.1 & & & & 490 & 8 & 12 & & 0.45 & 0.05 & 0.2 & 100 & 1.76 & 7.91 & \\
\hline HD4 & S76LD-10 & Llghtning Dock hot well & & & 8.40 & 1000 & 1160 & 1217 & 324 & 21 & & 0 & & & 145 & 474 & 83 & 9.9 & & & & & 138 & 2.04 & 0.40 & 4.88 \\
\hline \begin{tabular}{|l|} 
HD5 \\
\end{tabular} & SNM-10 & Lighting Dock Burgett Well \#1 & 95.2 & 8.70 & 9.06 & 1621 & 1136.6 & 1082 & 312 & 20 & 11.4 & 0.2 & 0.67 & 0.4 & & 492 & 88. & 11.1 & 0.2 & 0.33 & & & 145 & 5.13 & 8.86 & 0 \\
\hline HD7 & $P 2$ & Lightning Dock hot well & 85.0 & & 7.71 & & 1116 & 1232 & 333.6 & 23.5 & 22 & 0.5 & & & 106.8 & 497.1 & 88.3 & 12.6 & & 0.48 & & & 148 & 3.16 & & 43 \\
\hline HD8 & P3 & Llghtning Dock McCants Wel & 81.0 & & 8.16 & & 1024 & & 318.6 & 21.1 & 23.2 & $\begin{array}{ll}0.8 \\
\end{array}$ & 0.64 & 0.47 & 103.7 & 480 & 87.6 & 12 & 0.56 & 0.5 & & & 143 & 3.02 & & 16. \\
\hline & $P 4$ & $n g D_{c}$ & 71.0 & & 7.84 & & 1608 & 18 & 493.1 & 27.8 & 67.3 & 5.3 & & & 118.9 & 893.4 & 111.3 & 7.25 & & 0.42 & & & 116 & 3.77 & & 14.45 \\
\hline HD10 & $\frac{33}{532}$ & Llghtning Dac & 52.0 & 7.7 & & 2310 & & 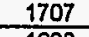 & 420 & 15 & 68 & \begin{tabular}{|l|}
4.9 \\
\end{tabular} & & & 140 & 830 & 91 & 6.6 & & 0.43 & 0.58 & 1.3 & 110 & 1.27 & 2.81 & \\
\hline HC & 90 & $8=$ & & & 7.70 & ror & 1680 & 1698 & & 15 & 68 & 4.9 & 0.98 & & 13 & 83 & & 6.6 & & 0.43 & & & 107 & 0.74 & 2.20 & 07 \\
\hline 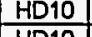 & S76LD-5 & Lightning D & & & 8.14 & 2260 & 1732 & & 440 & 31 & 71 & \begin{tabular}{|l|}
5.6 \\
\end{tabular} & 9 & & 105 & 88 & 11 & 3.2 & & 0.45 & & & 118 & 1.03 & 6.55 & 2.67 \\
\hline HD10 & S76LD-6 & & & & 8.07 & & 1786 & & 472 & 18 & 70 & 4.5 & 1.1 & & 11 & 83 & & 3.3 & 0.61 & 0.37 & & & 102 & 7.39 & 13.70 & 5.05 \\
\hline HD12 & 34 & Llghtning D & 33.5 & 8 & & 1960 & & 14 & 36 & 16 & 61 & \begin{tabular}{|l|}
6.6 \\
\end{tabular} & & & 21 & 540 & 15 & 6.4 & & 0.52 & 0.05 & 10 & 100 & 1.04 & 0.2 & \\
\hline HD12 & GS59534 & Llohthing Do & & & 8.00 & 1960 & 1380 & 1446 & 356 & 16 & 61 & 6.6 & \begin{tabular}{|l|}
0.72 \\
\end{tabular} & & 29 & 53 & 15 & 6.4 & & 0.52 & & & 100 & 0.88 & 0.07 & 4.80 \\
\hline HD12 & $S 76 L D-1$ & Llohtnling D & & & 8.25 & 1760 & 1295 & 15 & 410 & 17 & 64 & 6.8 & 0.8 & & 24 & 542 & 19 & 3.4 & 1.5 & 0.29 & & & 103 & 3.30 & 25.78 & 21.22 \\
\hline LA1 & 38 & Pueblo Car & 31.0 & 7.7 & & 766 & & 72 & 182 & 05 & 10 & & & & 428 & 43 & 16 & 2.5 & & & & 1.1 & 39 & 0.38 & 10.04 & \\
\hline LA & 38 & Pueblo Canyon \#7 Well & 30.5 & 7.8 & & 729 & & 6 & 170 & 0.4 & 9.8 & & & & 399 & 42 & 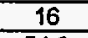 & 2.5 & & & & 1.2 & 39 & 0.65 & 8.29 & \\
\hline LI & PAL4 & we & 31.5 & & 8.07 & & 700 & 87 & 234.5 & 19.6 & 31.5 & 5.1 & & & 201.4 & 26 & 54. & 0.07 & & 0.24 & & & \begin{tabular}{|l|}
59 \\
\end{tabular} & 8.87 & & 25.14 \\
\hline TU & W86 & $\underline{w}$ & 31 & & 7.82 & & 73 & 9 & 244 & 18 & 26.4 & 4.. 0.420 & & & 346.6 & 245 & 52 & 5 & & 0.4 & & & 53 & 0.83 & & 36.19 \\
\hline $\mathrm{LL}$ & W69 & well & & & 8.70 & & 1168 & 13 & 416 & 3.9 & 1.4 & & & & 367.3 & 23 & 23 & 8.25 & & \begin{tabular}{|l|}
1.56 \\
\end{tabular} & & & \begin{tabular}{|l|l|}
39 \\
\end{tabular} & 0.77 & & 12.09 \\
\hline M! & DL-4-NM & Ya-Ta-Hey Well & & 20 & & & & 68 & 205 & 0.04 & 1.65 & 0.04 & 0.1 & 0.07 & 222 & 214.5 & 15. & 0.33 & & \begin{tabular}{|l|}
0.06 \\
\end{tabular} & 0 & & 23.6 & 2.81 & & \\
\hline$M$ & 43 & we & & 4 & 8.7 & & & & 3 & 2.1 & 13 & 0.09 & 0.11 & 2.4 & 189 & 560 & 12 & 1.5 & 0.16 & & 0.16 & & \begin{tabular}{|l|}
26 \\
\end{tabular} & 3.81 & 8.66 & \\
\hline MK4 & 43 & well & $\frac{0.4}{300}$ & 8.3 & 8.4 & 1600 & & 11 & 3 & 2 & 13 & 0.15 & 0.19 & 2.2 & 188 & 580 & 17 & 1.6 & 0.08 & & 0.1 & & 25 & 0.48 & 2.70 & \\
\hline MK7 & 299 & Navajo we & $\frac{36.0}{270}$ & 8.4 & & 594 & & 47 & 15 & 0.6 & 1.4 & 0.1 & & & 200 & 120 & 4.8 & 0.4 & & & 0.02 & & 22 & 1.58 & 3.23 & \\
\hline MK8 & DL-15-NM & $1 \operatorname{San} 7$ & 37.0 & & & & & 492 & 144 & 0.52 & 1.54 & & 0.16 & 0.08 & 180.56 & 138.11 & 5.9 & 0.4 & & 0.14 & 0.0 & & 20.5 & 2.88 & & \\
\hline MKS & 239 & well & 36.0 & 7.9 & & 1570 & & 1166 & 300 & 5 & 37 & \begin{tabular}{|l|}
8.9 \\
\end{tabular} & & & 29 & 410 & 98 & 1.1 & & & & & & 1.12 & 0.36 & \\
\hline
\end{tabular}




\begin{tabular}{|c|c|c|c|c|c|c|c|c|c|c|c|c|c|c|c|c|c|c|c|c|c|c|c|c|c|c|}
\hline$T E I D$ & SAMPLE & NAME & \begin{tabular}{|c|} 
TMP \\
$C$ \\
\end{tabular} & \begin{tabular}{|l|}
$p H$ \\
fineld \\
\end{tabular} & \begin{tabular}{|l|}
$\mathrm{pH}$ \\
lab \\
\end{tabular} & $\begin{array}{l}\text { COND } \\
\mathrm{uS} / \mathrm{cm}\end{array}$ & \begin{tabular}{|c|c|}
$T D S$ \\
m\&l \\
\end{tabular} & $\begin{array}{c}\text { TDS } \\
\text { (sum) } \\
\end{array}$ & $\begin{array}{c}\mathrm{Na} \\
\mathrm{mal} \\
\end{array}$ & \begin{tabular}{c|}
$K$ \\
$m g / L$ \\
\end{tabular} & $\begin{array}{cc}\mathrm{Ca} \\
\mathrm{mg} / \mathrm{L}\end{array}$ & \begin{tabular}{|c|}
$\mathrm{Mg}$ \\
$\mathrm{mog}$
\end{tabular} & \begin{tabular}{|c|}
$\mathbf{m g} / \mathrm{L}$ \\
\end{tabular} & $\begin{array}{c}s r \\
m g / 2\end{array}$ & $\mid \begin{array}{l}\mathrm{HCOO} \\
\text { moll }\end{array}$ & $\begin{array}{c}504 \\
\mathrm{~mol}\end{array}$ & $\begin{array}{c}a \\
m g h\end{array}$ & $\left|\begin{array}{c}F \\
m, /\end{array}\right|$ & $\begin{array}{l}B r \\
g / L\end{array}$ & \begin{tabular}{|c|}
$\mathbf{B}$ \\
$\mathrm{mo/L}$ \\
\end{tabular} & \begin{tabular}{|c|}
$\mathrm{Fe}$ \\
$\mathrm{mo} / \mathrm{L}$ \\
\end{tabular} & \begin{tabular}{|l|}
$\mathrm{NO3}$ \\
$\mathrm{mgl}$
\end{tabular} & $\left.\begin{array}{c}5102 \\
\alpha / 2\end{array}\right]$ & $\begin{array}{l}\text { CHRG } \\
\text { BAL } \%\end{array}$ & $\begin{array}{l}\text { COND } \\
\text { BAL } \%\end{array}$ & \begin{tabular}{|l} 
MAAS \\
BAL \%
\end{tabular} \\
\hline MK9 & 240 & well & 34.0 & 7.9 & & 1360 & & 1009 & 240 & 6 & 46 & 9.5 & & & 280 & 330 & $\frac{186}{86}$ & 1.9 & & & & 0.2 & 10 & & & \\
\hline$\frac{112}{112}$ & 296 & Pure Oil Navajo \#1 (Tohachi Well) & 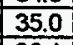 & \begin{tabular}{|l|}
9.1 \\
\end{tabular} & & 644 & & 484 & 140 & 1.1 & 1.8 & 0.2 & & & 184 & 130 & $\frac{\infty}{5.8}$ & 0.3 & & & & \begin{tabular}{|l|}
0.22 \\
0.22 \\
\end{tabular} & & $\frac{0.95}{2.66}$ & 3..35 & \\
\hline K12 & GS44727 & Pure Oil Navajo \#1 Well (Tohachl Well) & 36.1 & & 8.40 & 594 & 390 & 474 & 132 & \begin{tabular}{|l|l|}
0.6 \\
\end{tabular} & 1.4 & 0.1 & & & 198 & 115 & $\frac{3.0}{4.8}$ & 0.4 & & & & & & $\frac{2.00}{0.34}$ & $\frac{3.30}{1.76}$ & 21.62 \\
\hline MK12 & NM51 & Pure Oil Navajo \#1 Well (Tohachi Well) & & & 8.92 & & 376 & 503 & 152.4 & \begin{tabular}{|l|}
0.8 \\
0.8 \\
\end{tabular} & 1.2 & 0.01 & & & 181.8 & 142.2 & $\frac{4.0}{2.5}$ & 0.29 & & & & & $\frac{24}{21.5}$ & $\frac{0.34}{5.37}$ & & 33.79 \\
\hline MK17 & 243 & well & 32.5 & 8.5 & & 1500 & 906 & 1006 & 310 & \begin{tabular}{|l|}
2.7 \\
\end{tabular} & $\frac{1.6}{12}$ & 2.1 & & & $\frac{10.10}{223}$ & $\frac{1426}{380}$ & $\frac{2.5}{60}$ & $\begin{array}{ll}0.29 \\
0.8\end{array}$ & & 0.05 & 0.16 & 135 & $\frac{21.04}{15}$ & 3.79 & 4.50 & $\frac{3.1 .7}{1.06}$ \\
\hline MK18 & 422 & well & \begin{tabular}{|l|}
32.7 \\
\end{tabular} & \begin{tabular}{|l|}
8.6 \\
\end{tabular} & 8.5 & 2900 & & 2019 & 670 & \begin{tabular}{|l|}
2.3 \\
2.3 \\
\end{tabular} & $\frac{12 .}{7}$ & 9.2 & 0.07 & $\overline{0.28}$ & $\frac{223}{239}$ & 1000 & $\frac{\infty}{81}$ & 0.0 & 1.16 & .17 & $\frac{0.10}{0.04}$ & 1.30 & $\frac{75}{17}$ & $\frac{3.77}{4.60}$ & $\frac{4.30}{2.30}$ & 7.00 \\
\hline MK20 & 241 & well & \begin{tabular}{|l|}
32.0 \\
\end{tabular} & \begin{tabular}{|l|}
8.4 \\
\end{tabular} & & 9280 & 850 & 947 & 273 & \begin{tabular}{|l|}
2.7 \\
\end{tabular} & 11 & 3 & 0.06 & 0.20 & 284 & 306 & $\frac{10}{50}$ & $\begin{array}{l}0.1 \\
0.8 \\
\end{array}$ & .10 & 0.14 & $\frac{0.04}{.06}$ & & $\frac{11}{16}$ & $\frac{4.60}{1.04}$ & $\frac{2.30}{0.47}$ & 0.08 \\
\hline MK21 & 237 & well & \begin{tabular}{|l|}
31.5 \\
\end{tabular} & \begin{tabular}{|l|}
8.1 \\
\end{tabular} & & \begin{tabular}{|l|}
1220 \\
\end{tabular} & 802 & 929 & 230 & \begin{tabular}{|l|l|}
21 \\
3
\end{tabular} & 14 & $3: 8$ & & & $\frac{204}{270}$ & $\frac{30}{370}$ & $\frac{-30}{20}$ & $\frac{0.8}{0.4}$ & & 0.74 & .04 & 0.1 & $\frac{76}{18}$ & $\frac{1.04}{6.81}$ & $\frac{0.41}{9.07}$ & $\frac{0.06}{5.88}$ \\
\hline MK23 & 256 & well & \begin{tabular}{|l|}
30.5 \\
\end{tabular} & \begin{tabular}{|l|}
9.1 \\
\end{tabular} & 9 & 800 & & 682 & 200 & \begin{tabular}{|l|}
0.9 \\
\end{tabular} & $\frac{1.4}{1.4}$ & \begin{tabular}{|l|}
0.32 \\
0.32
\end{tabular} & 0.03 & $\overline{0.1}$ & $2 \pi 0$ & $\frac{310}{150}$ & $\frac{20}{6.5}$ & 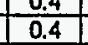 & 1.03 & & .04 & 0.7 & $\frac{78}{14}$ & 2.62 & 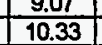 & \\
\hline OT5 & S76GART4 & Garton Well & \begin{tabular}{|l|}
33.7 \\
\end{tabular} & & 8.00 & \begin{tabular}{|l|}
10000 \\
\end{tabular} & 9086 & 9329 & 2225 & \begin{tabular}{|l|}
170 \\
\end{tabular} & 745 & \begin{tabular}{|l|}
176 \\
\end{tabular} & \begin{tabular}{|l|}
0.32 \\
\end{tabular} & & $\frac{\pi}{122}$ & 2854 & 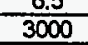 & 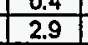 & 4.95 & 1.2 & & & $\frac{14}{30}$ & .62 & |l. & 2.68 \\
\hline OT5 & SAI & Garton Well & \begin{tabular}{|l|}
34.0 \\
\end{tabular} & & 7.12 & & 9140 & 8491 & 2032.4 & \begin{tabular}{|l|}
37 \\
\end{tabular} & \begin{tabular}{|l}
645.3 \\
\end{tabular} & \begin{tabular}{|l|}
186 \\
\end{tabular} & & & $\frac{120}{130.6}$ & 2958.7 & 2476.7 & 3.15 & 4.9 & $\frac{1.2}{0.75}$ & & & $\frac{30}{20}$ & $\frac{\frac{2.27}{1.14}}{x}$ & & $\frac{6.68}{.11}$ \\
\hline RA1 & GS13378 & Ojo Caliente Hot Spring Soda Spring & & & 7.2 & 3910 & & 3724 & 997 & \begin{tabular}{|l|l|l|}
29 \\
3
\end{tabular} & 25 & $\frac{100}{9}$ & & & 2180 & $\frac{2930.1}{162}$ & $\frac{240.8}{240}$ & $\frac{3.10}{16}$ & & & & & $\frac{20}{66}$ & $\frac{1.14}{0.66}$ & 7.90 & \\
\hline RA1 & HILLEERD & Ojo Caliente Hot Spring Soda Spring & & & & & & 3670 & 995.7 & 31.4 & 22.8 & 9.5 & 3.4 & 1.4 & $\frac{2153.5}{1.153}$ & 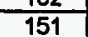 & 231.4 & \begin{tabular}{l|l}
5.2 \\
5.2
\end{tabular} & & 4.2 & & & 60.2 & .00 & & \\
\hline RA1 & $0 .-17$ & Ojo Callente Hot Spring Soda Spring & 27.3 & 6.86 & & 4220 & 3673 & 3673 & 950 & 28 & 20 & 7 & 3.5 & 1.74 & 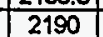 & $\frac{151}{154}$ & 240 & $\frac{0.4}{14.4}$ & 1.14 & & & & 0. & $\frac{1.40}{2.74}$ & $\overline{4.63}$ & .00 \\
\hline RA2 & NM21 & Ojo Callente Hot Spring Well & \begin{tabular}{|l|l|}
55.6 \\
\end{tabular} & & 7.74 & & 2576 & 12717 & 10018 & \begin{tabular}{|l|}
34.8 \\
3
\end{tabular} & $\frac{21.6}{11.6}$ & 4.8 & & & 2123.4 & $\frac{104}{196.9}$ & $\frac{240}{251.7}$ & 1.12 & l.14 & & & & $\frac{62}{74}$ & $\frac{2.14}{0.95}$ & 4.65 & $\frac{1.69}{3.69}$ \\
\hline RA2 & OC-18 & Ojo Caliente Hot Spring Well & 53.6 & 7.98 & & 4560 & 3724 & 3724 & 1000 & 28 & 8 & 3.9 & 3.64 & .65 & 2190 & 164 & 253 & $\begin{array}{ll}7.9 \\
7.9\end{array}$ & 01 & & & & & 2.07 & & \\
\hline RA2 & $\infty-25$ & Ojo Caliente Hot Spring Well & 54.2 & 7.14 & & 4440 & 3618 & 3618 & 940 & 30 & 17 & \begin{tabular}{|l|l|}
5.4 \\
\end{tabular} & \begin{tabular}{|l|}
3.17 \\
.07
\end{tabular} & 28 & $\frac{2100}{2130}$ & $\frac{107}{160}$ & $\frac{200}{246}$ & $\frac{17.5}{17.3}$ & .22 & & & & & & & \\
\hline RA2 & $00-4$ & Ojo Caliente Hot Spring Well & \begin{tabular}{|l|}
54.0 \\
\end{tabular} & \begin{tabular}{|c|}
6.75 \\
6.75 \\
\end{tabular} & & 3900 & $\begin{array}{lll}3010 \\
3344 \\
\end{array}$ & 3344 & 927 & \begin{tabular}{|l|l}
51.8 \\
27.8
\end{tabular} & 7.3 & \begin{tabular}{|l|}
2.9 \\
\end{tabular} & \begin{tabular}{|l|l|}
5.5 \\
5.5
\end{tabular} & 0.48 & $\frac{2130 \mid}{1870}$ & $\frac{100}{195}$ & $\frac{246}{242}$ & $\frac{7.3}{20.8}$ & & & & & $\frac{65}{43.4}$ & & & \\
\hline RA3 & GS13193 & Ojo Caliente Arsenic Hot Spring & \begin{tabular}{|l|l|}
45.0 \\
\end{tabular} & & 7.10 & 3930 & & 3627 & $\begin{array}{l}921 \\
928 \\
\end{array}$ & $\frac{21.0}{30}$ & $\frac{1.5}{25}$ & \begin{tabular}{|l|}
8.9 \\
\end{tabular} & 0.0 & 0.48 & $\frac{1810}{2160}$ & $\frac{150}{156}$ & $\frac{242}{238}$ & & & & & & 43.4 & & & \\
\hline RA3 & NM19 & Ojo Caliente Arsenic Hot Spring & 43.5 & & 7.03 & & 2652 & 3739 & 993.1 & 36 & 21.6 & 7.6 & 3.86 & .28 & $\frac{2100}{2172.2}$ & $\frac{100}{187.3}$ & $\frac{200}{235.4}$ & $\frac{10}{4.7}$ & 42 & & & & & & & 0.98 \\
\hline $\mathrm{RA3}$ & $\infty-14$ & Ojo Caliente Arsenio Hot Spring & 38.3 & 7.44 & & 4200 & 3665 & 3665 & 950 & 30 & 22 & \begin{tabular}{|l|}
8.1 \\
\end{tabular} & 3.59 & 1.49 & 2180 & 157 & 238 & 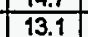 & . 493 & & & & $\frac{05}{59}$ & & & \\
\hline RA3 & oc-5 & Ofo Callente Arsenic Hot Spring & 43.5 & 6.60 & & 4000 & 3131 & 3130 & 914 & 31 & 22 & \begin{tabular}{|l|}
7.8 \\
\end{tabular} & \begin{tabular}{|l|}
0.38 \\
\end{tabular} & $\frac{1.70}{1.22}$ & $\frac{2100}{1690}$ & $\frac{107}{150}$ & $\frac{.000}{235}$ & $\frac{0.1}{6.1}$ & & & & & 61 & 21 & & \\
\hline RA3 & ST6ARSEN & Ojo Callente Arsenic Hot Spring & 38.3 & 7.10 & & & & 3667 & 1000 & 30.6 & 28 & 7.8 & \begin{tabular}{|l|l|}
4.3 \\
\end{tabular} & & 2135 & $\frac{100}{149}$ & $\frac{200}{242}$ & 0.1 & .15 & & & & & $\frac{05}{45}$ & & \\
\hline RA5 & $O C-13$ & Ojo Caliente HS Lithia Spring & 41.9 & 7.08 & & 4490 & 3630 & 3630 & 950 & 30 & 26 & \begin{tabular}{|l|}
9.5 \\
\end{tabular} & \begin{tabular}{|l|}
3.57 \\
\end{tabular} & 1.48 & 2160 & $\frac{19}{151}$ & $\frac{2427}{227}$ & $\frac{1.0}{11.8}$ & .02 & & & & 5 & & & 0.01 \\
\hline RA5 & $0 c-2$ & Ojo Caliente HS Lithia Spring & 38.0 & \begin{tabular}{|l|l|}
6.55 \\
\end{tabular} & & \begin{tabular}{|l|}
3900 \\
\end{tabular} & 3475 & 3295 & 687 & 28.5 & 23.6 & \begin{tabular}{|l|}
3.7 \\
8.7
\end{tabular} & \begin{tabular}{|l|}
2.37 \\
2.7 \\
\end{tabular} & $\frac{1.40}{1.16}$ & & $\frac{101}{173}$ & $\frac{227}{227}$ & $\frac{71.6}{13.5}$ & & & & & $\frac{31}{58.5}$ & $\frac{96}{111}$ & $\frac{13}{58}$ & \\
\hline RAG & GS13192 & Ojo Caliente HS Sodium Sulfate Spring & 40.6 & & 6.90 & \begin{tabular}{|l|}
3920 \\
\end{tabular} & & 3633 & 933 & 34 & $\frac{24}{24}$ & 7.6 & & & 2160 & 156 & $\frac{24}{238}$ & & & & & & $\frac{0.04}{63}$ & 27 & 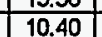 & \\
\hline RAG & NM20 & Ojo Caliente HS Sodium Sulfate spring & \begin{tabular}{|l|l|}
41.1 \\
\end{tabular} & & 7.20 & & 2668 & 3795 & 993.1 & $\frac{4}{36}$ & 20.3 & \begin{tabular}{|l|}
7.4 \\
\end{tabular} & & & $\frac{2100}{2245.4}$ & 187.3 & $\frac{308}{237.2}$ & & & & & & $\frac{63}{66}$ & & & .25 \\
\hline RAG & $00-16$ & Ojo Caliente HS Sodium Sulfate Spring & 41.3 & 7.02 & & 4250 & 3628 & 3627 & 950 & 28 & 20 & 7.1 & 3.64 & 49 & 2150 & 153 & 237 & & & 41 & & & $\frac{0}{61}$ & & & \\
\hline RAG & OC-3 & Ojo Caliente HS Sodium Sulfate Spring & 40.0 & 6.20 & & 4100 & 3533 & 3533 & 866 & 31.3 & 20.6 & \begin{tabular}{|l|l|}
6.6 \\
\end{tabular} & 2.4 & $\frac{10}{4}$ & & 100 & $\frac{251}{237}$ & & & & & & \begin{tabular}{|c|c|}
64.3 \\
6
\end{tabular} & & & \\
\hline RA7 & $\infty-1$ & Ojo Caliente Hot Spring Iron Spring & 43.0 & 6.40 & & 4100 & 3578 & 3578 & 953 & 29 & $\frac{21}{21}$ & \begin{tabular}{|l|l|}
6.6 \\
\end{tabular} & \begin{tabular}{|l|}
2.2 \\
2.2 \\
\end{tabular} & 1.14 & & 17 & $\frac{201}{229}$ & & & & & & $\frac{\mid 40.5}{58.9} \mid$ & & & \\
\hline RA7 & OC-15 & ojo Caliente Hot S & 42.2 & 7.26 & & 4250 & 3674 & 3674 & 950 & $\frac{-20}{30}$ & $\frac{21}{22}$ & \begin{tabular}{|l|l|}
8.1 \\
\end{tabular} & \begin{tabular}{|l|}
3.38 \\
3
\end{tabular} & 1.45 & $\frac{20}{21}$ & & & & 1.02 & & & & & & & \\
\hline RA7 & S76IRON1 & Ojo Caliente Hot $\mathrm{S}$ & & & 9.09 & 3300 & 2438 & 30141 & 993 & $\frac{5}{36}$ & $\frac{24}{6}$ & \begin{tabular}{|l|}
7.7 \\
\end{tabular} & $\mid \frac{2.30}{2.7}$ & 1.45 & & & $\frac{2}{2}$ & 19.7. & 1.02 & 421 & & & $\frac{61}{71}$ & & & 81 \\
\hline RA7 & S761RON2 & ojo Callente Hot $\mathrm{S}$ & 42,8 & & & & & & 980 & 31.5 & 29 & \begin{tabular}{|l|l|}
8.5 \\
\end{tabular} & \begin{tabular}{|l|}
-3.3 \\
\end{tabular} & & & & & & 166 & & & & $\frac{11}{55}$ & & & \\
\hline$R A B$ & $0 \mathrm{C}-26$ & Statue s & 29.2 & & & 1660 & 1386 & $\frac{25}{13}$ & 160 & 15 & $\frac{25}{126}$ & \begin{tabular}{|l|l|}
48 \\
48
\end{tabular} & & & $\frac{019}{649}$ & & $\frac{10}{10}$ & 1.44 & & $41 \|$ & & & & & & \\
\hline RA9 & OC-20 & Ojo Callente Hot & 34.3 & 6.88 & & 3000 & & & $\frac{\pi}{62}$ & $\overline{1}$ & 70 & 21.6 & \begin{tabular}{|l|}
2.04 \\
\end{tabular} & 1.34 & $14 \div>>$ & & & 8.4 & $\overline{0.83}$ & |0.91| & & & \begin{tabular}{|l|l|}
5 \\
\end{tabular} & & & \\
\hline RA9 & PH65-524 & Ojo Callente Hot Spring Well \#1 & & & 7.80 & 2375 & 1515 & 17 & 49 & 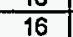 & 40 & \begin{tabular}{|l|l|}
216 \\
16
\end{tabular} & & & & & & & & & & & & & & \\
\hline RA11 & NM18 & Jo allente not & 25.5 & & $\frac{1.00}{6020}$ & & $\frac{1010}{1072}$ & $\frac{716}{14}$ & $\frac{456}{183 .}$ & \begin{tabular}{|c|}
16.8 \\
16.8
\end{tabular} & $\frac{40}{138.9}$ & \begin{tabular}{|l|}
56.5 \\
\end{tabular} & 0.54 & 0.85 & $\frac{91}{695.6}$ & 21 & $\frac{74}{107}$ & 1.31 & 0.97 & $\mid 0.33$ & & & 21.5 & & & \\
\hline$\overline{\mathrm{RA12}}$ & $\mathrm{PH} 65-522$ & Ojo Call & & & $\overline{7.8}$ & & & & 10 & - & 80 & 29 & & & 274 & & 8 & 0.55 & & & & & & $\frac{2.20}{35.29}$ & & $\frac{100}{46}$ \\
\hline$\overline{a x}>>1$ & & & $\overline{070}$ & & & & 57 & & & 330 & 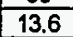 & & $3.7 .7-3$ & & & & & (1) & $3.56>>>$ & $\overline{5.4}$ & & & & & & \\
\hline $\mathrm{SA}$ & & & & & & & & 644 & 19 & & & & & & & & & & & & & & & & & \\
\hline & $B_{8}$ & & & & & & & & & & & & & & 19 & & 26 & 6.8 & 7.83 & 17.9 & & & 76 & & 14.1 & 17.93 \\
\hline$\overline{S A:}$ & $\overline{B A}$ & & 294.0 & & & 910 & & 55 & 15 & 20 & 3. & & & 0 & 21 & 49 & $\frac{25}{26}$ & 6.6 & 7.8 & & & & 720 & 0.46 & $13.48 \mathrm{~d}$ & 15.70 \\
\hline $\mathrm{SAC}$ & $B A$ & & & 7.20 & & 85 & 4644 & 54 & 15 & 25 & 4.3 & 0.19 & 20. & & 23 & 47 & 25 & 9.6 & 7.01 & 18 & & & 680 & & 13.82 & 53 \\
\hline SAS & $B A$ & & & & & & 45 & & $155^{2}$ & 2 & & & 1 & & 22 & 49 & 25 & 9.4 & 7 & 18 & & & 640 & 1.24 & & 16.57 \\
\hline$\overline{S A G}$ & & Jer & & & & 100 & 53 & 60 & $\overline{18}$ & 23 & & & 25 & & 85 & 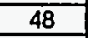 & 31 & 6.4 & 36 & & & & 620 & 0.20 & & 14.31 \\
\hline SAE & $\mathrm{BA}$ & Je & & & & & & $\frac{6 x}{6}$ & 18 & 235 & 1.1. & & 23.2 & & & 48 & 31 & 6.6 & 9.42 & & & & 600 & 27 & 12.80 & \\
\hline SA7 & $\mathrm{VA}$. & Jemez/GRIWC 23- & 23 & & & 30 & & 18 & 58 & 10 & 46 & 0.45 & & 1. & 3 & 9 & 99 & 13.8 & 27 & & & & 450 & 0.72 & & \\
\hline SAB & $\mathrm{BA}$ & & & & & 10 & 50 & 56 & 19 & 31 & 13.1 & \begin{tabular}{|l|l}
0. \\
\end{tabular} & \begin{tabular}{|l|}
25.6 \\
\end{tabular} & & 1 & 48 & 33 & 6.9 & 10.9 & $\sqrt{2}$ & & & & 4 & 12 & \\
\hline SA9 & & Jeme: & 214.0 & & & & 8580 & $8:$ & & 47 & 80.5 & 2. & \begin{tabular}{|l|}
28.3 \\
\end{tabular} & & 36 & 3 & 43 & 6.9 & 14 & & & & 395 & 54 & & \\
\hline SA12 & & HS Jer & 73.3 & & & & 25 & 2 & 58 & 62 & 121 & 4.55 & 6.8 & & 69 & 39 & 9 & 6.4 & 2.2 & & & & \begin{tabular}{|l|l|}
89 \\
\end{tabular} & 5.38 & 99.74 & \\
\hline SA12 & VA. & Jemez HS Jemez Sprir & 72.2 & & & 46 & 30 & 26 & 63 & 61 & 126 & 4.23 & \begin{tabular}{|l|}
8.5 \\
\end{tabular} & 1.22 & 79 & 41 & 94 & 5.38 & 2.6 & 6. & & & 94 & 3.36 & 21 & 12.30 \\
\hline SA1 & VA-15 & Jemez HS Jemez Springs $\# 1$ Well $152 \mathrm{~m}$ & 60.5 & 6. & & 170 & 1140 & 11 & 18 & 29.9 & 120 & 9.31 & \begin{tabular}{|l|}
2.27 \\
\end{tabular} & 0.4 & 4 & 38 & $24:$ & 3.3 & & 2.2 & & & 24 & 0.73 & 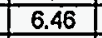 & 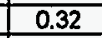 \\
\hline SA12 & $V A$-19 & Jemez HS Jemez Springs \#1 Well $24 \mathrm{~m}$ & 68.0 & 6. & & 3300 & 22 & 22 & 546 & 61.6 & 122 & 5.76 & 6.96 & 0.54 & 642 & 45 & 705 & 4.42 & & 1 & & & 70 & 2.05 & 0.29 & 0.21 \\
\hline
\end{tabular}




\begin{tabular}{|c|c|c|c|c|c|c|c|c|c|c|c|c|c|c|c|c|c|c|c|c|c|c|c|c|c|c|}
\hline SITE ID & SAMPLE & NAME & $\begin{array}{c}T M P \\
C \\
\end{array}$ & \begin{tabular}{|l|}
$p H$ \\
feld \\
\end{tabular} & \begin{tabular}{|l|}
$\mathrm{PH}$ \\
$\mathrm{Lab}$
\end{tabular} & $\begin{array}{l}\text { COND } \\
\mu S / \mathrm{cm}\end{array}$ & $\begin{array}{l}D S S \\
\text { moll }\end{array}$ & $\begin{array}{c}\text { TDS } \\
\text { (sum) }\end{array}$ & $\begin{array}{c}\mathrm{Na} \\
\mathrm{mgl}\end{array}$ & \begin{tabular}{c|}
$k$ \\
$m g / L$ \\
\end{tabular} & $\begin{array}{c}\mathrm{Ca} \\
\mathrm{mg} / \mathrm{h}\end{array}$ & \begin{tabular}{|c|}
$\mathrm{Mg}$ \\
$\mathrm{mg} / \mathrm{L}$
\end{tabular} & $\begin{array}{c} \\
m \sigma^{\prime \prime}\end{array}$ & \begin{tabular}{|c|}
$s r$ \\
$m g h$ \\
\end{tabular} & $\begin{array}{l}\mathrm{HCO3} \\
\mathrm{mg} / \mathrm{L}\end{array}$ & $\begin{array}{l}504 \\
\mathrm{~mol} / \mathrm{L}\end{array}$ & $\begin{array}{c}c \\
m g / L\end{array}$ & \begin{tabular}{|c|}
$F / L$ \\
$m q L$
\end{tabular} & $\begin{array}{l}B r \\
g / L\end{array}$ & \begin{tabular}{|c|}
$B$ \\
$m q / L$ \\
\end{tabular} & $\begin{array}{c}F_{\theta} \\
m g / L\end{array}$ & \begin{tabular}{|l|} 
NO3 \\
mo/L
\end{tabular} & \begin{tabular}{|c|}
$\mathrm{SiO2}$ \\
$\mathrm{g} / \mathrm{L}$
\end{tabular} & $\begin{array}{l}\text { CHRG } \\
\text { BAL \& }\end{array}$ & \begin{tabular}{|l|} 
COND \\
BAL \&
\end{tabular} & $\begin{array}{l}\text { MASS } \\
\text { BAL \% }\end{array}$ \\
\hline SA12 & VA-21 & Jemez HS Jemez Springs \#1 Well $152 \mathrm{~m}$ & 61.0 & 6.55 & & 1830 & 1200 & 1229 & 193 & 35.4 & 122 & 9.25 & 3.6 & 0.4 & 492 & 49.9 & 281 & 3.5 & & 2.5 & & & 36 & 1.58 & 8.87 & 2.38 \\
\hline SA12 & VA-25 & Jemez HS Jemez Springs \#1 Well $24 \mathrm{~m}$ & 73.3 & 6.55 & & 3500 & 2560 & 2555 & 610 & 68 & 180 & 4.6 & 8.4 & \begin{tabular}{|l|l}
0.86 \\
\end{tabular} & 705 & 53 & 836 & 3.52 & & 6.8 & & & 79 & 3.24 & 11.04 & 0.19 \\
\hline SA13 & VA-123 & Jemez HS Travertine Mound Spring & 72.6 & 6.59 & & 4360 & 2550 & 2545 & 596 & 62 & 129 & 4.49 & 6.1 & \begin{tabular}{|l|l|}
0.57 \\
\end{tabular} & 697 & 38 & 906 & 6.3 & 2.3 & 7.02 & & & 90 & 3.99 & 19.24 & 0.20 \\
\hline SA13 & VA-142 & Jemez HS Travertine Mound Spring & 72.9 & 6.20 & & 4740 & 2630 & 2634 & 610 & 58 & 132 & 4.3 & 8.6 & 1.22 & 757 & 40 & 917 & 4.31 & 2.7 & \begin{tabular}{|l|l|}
6.7 \\
\end{tabular} & & & 92 & 4.16 & 23.57 & 0.15 \\
\hline SA13 & VA-17 & Jemez HS Travertine Mound Spring & 72.0 & 6.66 & & 4100 & 2600 & 2599 & 612 & 70.3 & 114 & 4.48 & 8.46 & 0.54 & 714 & 43.2 & 936 & 5.05 & & 7.9 & & & 83 & 4.75 & 12.91 & 0.04 \\
\hline SA13 & VA-66 & Jemez HS Travertine Mound Spring & 72.0 & 6.66 & & 3400 & 1700 & 2251 & 558 & 62 & $\cdot 122$ & 5.4 & 9 & \begin{tabular}{|l|}
0.59 \\
\end{tabular} & 436 & 42.4 & 910 & 4 & 2.6 & 7 & & & 92 & 0.36 & 0.88 & 32.41 \\
\hline SA13 & VA-7 & Jemez HS Travertine Mound Spring & 70.0 & 6.28 & & 4200 & 7580 & 2579 & 614 & 75.2 & 182 & 4.56 & 8.2 & 0.6 & 723 & 36.1 & 829 & 5.21 & & 7.8 & & & 93 & 4.00 & 6.47 & 65.98 \\
\hline SA13 & VA-71 & Jemez HS Travertine Mound Spring & 72.0 & 6.12 & & 3900 & 2520 & 2515 & 540 & 73 & 135 & 4.75 & 8.7 & 0.34 & 712 & 41 & 894 & 4.4 & 2.8 & 7.19 & & & 92 & 5.94 & 13.47 & 0.19 \\
\hline SA14 & 272 & Jemez HS Soda Spring & 65.5 & 7.2 & & 3560 & 2150 & 2476 & 572 & 70 & 138 & 6.6 & & & 735 & 49 & 795 & 5.2 & & 11 & 0.03 & 0.8 & 93 & 2.39 & 4.21 & 15.15 \\
\hline SA16 & 267 & Jemez Hot Springs & 49.0 & 6.7 & & 3550 & & 2548 & 550 & 68 & 170 & 9.2 & & & 800 & 49 & 800 & 4.6 & 1 & 6.5 & 1 & 0.04 & 89 & 2.85 & 1.68 & \\
\hline SA16 & 269 & Jemez Hot Springs & 58.0 & 7.1 & & 3460 & & 2350 & 510 & 63 & 160 & 6.6 & 7.8 & & 773 & 43 & 700 & 4.4 & 3 & 7.4 & 0.45 & 0.35 & 71 & 0.20 & 3.33 & \\
\hline SA16 & JEMEZ 5 & Jemez Hot Springs & 56.0 & & 6.89 & & 1952 & 2378 & 603.5 & \begin{tabular}{|l|}
63.7 \\
\end{tabular} & 129.1 & 5.2 & & & 685.8 & 53 & 740.6 & 4.62 & & $\begin{array}{l}1.7 \\
7 \\
\end{array}$ & & & \begin{tabular}{|l|}
85.9 \\
\end{tabular} & 1.86 & & 21.85 \\
\hline SA16 & JEMEZ6 & Jemez Hot Springs & 53.0 & & 6.90 & & 1884 & 2373 & 608.8 & 64.1 & 103 & 5.3 & & & 691.9 & 53 & 748.4 & 4.64 & & \begin{tabular}{|l|}
6.9 \\
\end{tabular} & & & \begin{tabular}{|l|}
87.4 \\
\end{tabular} & 0.15 & & $\frac{21.00}{25.98}$ \\
\hline SA16 & JEMEZ7 & Jemez Hot Springs & 74.0 & & 7.01 & & 2156 & 2547 & 668.8 & \begin{tabular}{|l|}
70.8 \\
\end{tabular} & 97.2 & 4.5 & & & 738.3 & & 859.7 & 5.2 & & \begin{tabular}{|l|}
7.2 \\
\end{tabular} & & & \begin{tabular}{|l|}
95.4 \\
\end{tabular} & 0.69 & & 18.14 \\
\hline SA18 & 91TDI1 & Jemez Pueblo \#1 Well & 57.8 & 6.90 & & & 3366 & 3853 & 1187.5 & 58.6 & 67.6 & 3.19 & 4.7 & \begin{tabular}{|l|}
3.19 \\
\end{tabular} & 1047 & 347.3 & 1086.4 & 6.8 & & 6.78 & & & \begin{tabular}{|l|}
34.9 \\
\end{tabular} & \begin{tabular}{|l}
.00 \\
1.90
\end{tabular} & & $\frac{0.94}{14.47}$ \\
\hline \begin{tabular}{|l|} 
SA18 \\
\end{tabular} & 91TDI3 & Jemez Pueblo \#1 Well & 58.2 & 6.69 & & & 3349 & 4036 & 1043.5 & \begin{tabular}{|l|}
637 \\
\end{tabular} & 57.5 & 8.2 & 5.1 & 3.1 & 789.5 & 268.4 & 1191.8 & 7.3 & & 7.14 & & & 17.6 & 11.38 & & 20.52 \\
\hline \begin{tabular}{|l|} 
SA18 \\
\end{tabular} & JP91-2 & Jemez Pueblo \# 1 Well & 57.8 & 7.00 & & 5560 & 3947 & 3946 & 1148 & 67 & 69.5 & 12.7 & 6.09 & 2.76 & $\$ 132$ & 254 & 1196 & 6.68 & 3.33 & 8.46 & & & 39 & \begin{tabular}{|c|}
0.76 \\
0.76 \\
\end{tabular} & 2.71 & 0.04 \\
\hline \begin{tabular}{|l|} 
SA19 \\
\end{tabular} & VA-10 & Jemez HS Gazebo Spring & 55.0 & 7.01 & & 4200 & 2660 & 2660 & 656 & 74.2 & 152 & 5.4 & 10.1 & 0.56 & 711 & 40.9 & 904 & \begin{tabular}{|l|}
5.19 \\
\end{tabular} & & \begin{tabular}{|l|}
7.9 \\
\end{tabular} & & & 93 & 2.11 & 4.93 & 0.01 \\
\hline SA19 & VA-122 & Jemez HS Gazebo Spring & \begin{tabular}{|l|}
74.9 \\
\end{tabular} & 6.57 & & 4380 & 2540 & 2536 & 583 & 62 & 126 & 4.57 & 6.8 & 0.57 & 698 & 39 & 909 & 6.4 & 2.2 & 7.11 & & & 99 & 5.02 & 21.00 & 0.17 \\
\hline SA19 & VA-143 & Jemez HS Gazebo Spring & \begin{tabular}{|l|l|}
74.7 \\
\end{tabular} & 6.50 & & 4460 & 2640 & 2637 & 610 & 58 & 130 & 4.55 & 8.4 & 1.18 & 746 & 42 & 932 & 4.64 & 2.7 & \begin{tabular}{|l|l|}
6.4 \\
\end{tabular} & & & 91 & 4.69 & 19.02 & 0.12 \\
\hline SA19 & VA-18 & Jemez HS Gazebo Spring & 36.0 & 7.51 & & 4250 & 2700 & 2704 & 690 & 74 & 115 & 4.52 & 9 & 0.6 & 699 & 45.4 & 958 & 5.19 & & 8 & & & 85 & 0.82 & 7.46 & 0.14 \\
\hline SA20 & 247 & Kaseman \#2 Well (Zia Hot Well) & 54.4 & 7.3 & & 15300 & & 11888 & 3600 & 88 & 350 & 56 & & & 1460 & 3300 & 3000 & 2.8 & & & & 0.2 & 39 & 0.98 & 18.25 & \\
\hline \begin{tabular}{|l|} 
SA20 \\
\end{tabular} & 252 & Kaseman \#2 Well (Zia Hot Well) & 52.0 & 6.8 & & 15700 & & 11859 & 3500 & 88 & 350 & 61 & & & 1410 & 3300 & 3100 & 3.4 & 8.1 & 7.5 & 1.4 & 0.09 & 30 & 0.72 & 12.73 & \\
\hline \begin{tabular}{|l|} 
SA20 \\
\end{tabular} & 88TDI1 & Kaseman $\# 2$ Well (Zia Hot Well) & \begin{tabular}{|l|}
53.0 \\
\end{tabular} & 7.31 & 6.70 & & 10720 & 11339 & 3006 & 66.8 & 291.5 & 50.3 & 3.55 & 8.01 & 1667 & 3283 & 2919.8 & 2.97 & & 6.25 & & & 33.9 & 7.98 & & 5.77 \\
\hline SA20 & $E$ & Kaseman \#2 Well (Zia Hot Well) & \begin{tabular}{|l|}
52.0 \\
\end{tabular} & & 6.87 & & 11300 & 12229 & 3720.4 & \begin{tabular}{|l|}
92.7 \\
\end{tabular} & 417.8 & 73.4 & & & 1464.4 & 3343 & 3067.4 & 4.8 & & \begin{tabular}{|l|}
6.96 \\
\end{tabular} & & & \begin{tabular}{|l|}
37.9 \\
\end{tabular} & 2.88 & & 8.22 \\
\hline \begin{tabular}{|l|} 
SA20 \\
\end{tabular} & VA-125 & Kaseman \#2 Well (Zia Hot Well) & 53.0 & 6.98 & & 15700 & 11400 & 11351 & 3080 & 63.4 & 364 & 62.8 & 5.2 & 8.7 & 1398 & 3338 & 2984 & 2.47 & 3.4 & \begin{tabular}{|l|}
7.8 \\
\end{tabular} & & & 33 & 5.02 & 1.83 & 0.43 \\
\hline SA2O & VA.34 & Kaseman \#2 Well (Zia Hot Well) & 56.0 & 6.29 & & 16600 & 12200 & 11343 & 2650 & 66.7 & 302 & 90 & 6.7 & 9 & 1440 & 3740 & 3000 & 2.4 & & \begin{tabular}{|l|l|}
6.52 \\
\end{tabular} & & & 30 & 13.95 & 15.29 & $\frac{0.72}{7.02}$ \\
\hline SA20 & VA-53 & Kaseman \#2 Well (Zla Hot Well) & 54.0 & 6.53 & & 16000 & 11700 & 11667 & 3440 & 77 & 321 & 61 & 6 & 4.75 & 1068 & 3430 & 3210 & 4.51 & 4.2 & \begin{tabular}{|l|l|}
7.41 \\
\end{tabular} & & & 33 & 1.74 & 8.51 & 0.28 \\
\hline \begin{tabular}{|l|} 
SA20 \\
\end{tabular} & VA-67 & Kaseman \#2 Well (Zia Hot Well) & 53.0 & 6.72 & & 15800 & 11300 & 11311 & 3180 & 64 & 320 & 71.5 & 6.3 & \begin{tabular}{l|l}
9.55 \\
9.5
\end{tabular} & 9400 & 3280 & 2930 & 3.8 & 4.2 & 6.6 & & & 35 & 3.32 & 3.13 & 0.10 \\
\hline \begin{tabular}{|l|} 
SA20 \\
\end{tabular} & VA-74 & Kaseman \#2 Well (Zia Hot Well) & 53.0 & 6.40 & & 15600 & 12800 & 12897 & 3700 & 54 & 354 & 21.8 & \begin{tabular}{|l|l|}
5.52 \\
\end{tabular} & 6.87 & 1400 & 4100 & 3210 & 2.67 & 1.1 & 6.9 & & & $\frac{\pi}{34}$ & 4.26 & 17.14 & 0.76 \\
\hline \begin{tabular}{|l|} 
SA21 \\
\end{tabular} & VA-16 & Jemez HS Buddhist Spring & 50.0 & 6.59 & & 3300 & 2550 & 2177 & 494 & 57.8 & 128 & 7.5 & \begin{tabular}{|l|l|}
6.06 \\
\end{tabular} & 0.52 & 708 & 40.6 & 653 & 3.76 & & 5.7 & & & 72 & 0.34 & 6.50 & 14.63 \\
\hline SA21 & VA-B & Jemez HS Buddhlst Spring & 49.0 & 6.38 & & 3300 & 2160 & 2161 & 458 & 53 & 154 & 9.57 & \begin{tabular}{|l|l|}
7.56 \\
\end{tabular} & 0.56 & 697 & 37.6 & 653 & 3.86 & & 5.7 & & & 81 & 0.03 & 6.51 & 0.04 \\
\hline SA22 & VA-12 & Jemez HS Marsh Spring & 49.0 & 6.35 & & 4100 & 2620 & 2620 & 609 & 70 & 129 & 7.82 & 8.18 & 0.64 & 738 & 41.8 & 903 & 4.56 & & 7.5 & & & 100 & 2.82 & 10.84 & 0.02 \\
\hline SA23 & JEMEZ2 & Jemez/Soda Dam Hot Spring & 47.0 & & 7.06 & & 3496 & 4641 & 1087.9 & 185 & 285.6 & 24.1 & & & 1526.6 & 50 & 1420.2 & 3.5 & & \begin{tabular}{|l|}
12.5 \\
12
\end{tabular} & & & \begin{tabular}{|l|}
46.4 \\
\end{tabular} & $\frac{2.02}{1.46}$ & & $\frac{0.06}{32.76}$ \\
\hline SA23 & VA-109 & Jemez/Soda Dam Hot Spring & 46.8 & 6.21 & & 7090 & 4570 & 4564 & 1030 & 160 & 245 & 17.4 & 12.7 & 1.39 & 1458 & 34 & 1536 & 3.28 & 4.07 & \begin{tabular}{|l|}
15.7 \\
\end{tabular} & & & 46.8 & 2.82 & 9.15 & 0.12 \\
\hline SA23 & VA- 132 & Jemez/Soda Dam Hot Spring & 47.0 & 7.19 & & 6800 & 4590 & 4585 & 980 & 186 & 315 & \begin{tabular}{|l|}
22.7 \\
\end{tabular} & 15.8 & 1.4 & 1488 & 35 & 1477 & 3.51 & 4.6 & \begin{tabular}{|l|l|}
13.9 \\
\end{tabular} & & & 42 & 0.19 & 1.06 & 0.11 \\
\hline SA23 & VA-140 & Jemez/Soda Dam Hot Spring & 46.8 & 6.71 & & 7300 & 4570 & 4570 & 960 & 160 & 342 & 21.9 & 13.8 & 2.84 & 1488 & 34 & 1480 & 3.33 & 4.6 & 12.1 & & & 47 & 0.23 & 8.53 & 0.01 \\
\hline SA23 & VA-26 & Jemez/Soda Dam Hot Spring & 47.0 & 5.52 & & 6600 & 4630 & 4625 & 920 & 177 & 429 & 21.4 & 13,6 & 2.02 & 1490 & 49.4 & 1460 & 3.57 & & \begin{tabular}{|l|}
12.8 \\
\end{tabular} & & & 46 & 2.12 & 5.64 & 0.11 \\
\hline SA23 & VA-51 & Jemez/Soda Dam Hot Spring & 47.0 & 6.35 & & 5900 & 4150 & 4153 & 990 & 183 & 314 & 24 & 13.5 & 0.89 & 1000 & 39.1 & 1520 & 3.55 & 3.84 & 15 & & & 46 & 5.51 & 14.16 & 0.07 \\
\hline SA23 & VA-6 & Jemez/Soda Dam Hot Spring & 47.0 & 6.20 & & & 4014 & 4014 & 1010 & 174 & 328 & 26 & \begin{tabular}{|l|}
13.2 \\
\end{tabular} & 1.38 & 886 & 37 & 1480 & 4.1 & & \begin{tabular}{|c|}
11.5 \\
\end{tabular} & & & 43 & 9.17 & & 0.00 \\
\hline SA23 & VA.64 & Jemez/Soda Dam Hot Spring & 47.0 & 6.28 & & 5600 & 4200 & 4199 & 825 & 120 & 300 & 25 & \begin{tabular}{|l|}
13.7 \\
\end{tabular} & 1.48 & 1250 & 36.1 & 1560 & 2.8 & 6.7 & \begin{tabular}{|l|l|}
13.9 \\
\end{tabular} & & & 44 & 6.06 & 3.55 & 0.03 \\
\hline SA23 & VA-70 & Jemez/Soda Dam Hot Spring & 47.0 & 6.28 & & 6700 & 4380 & 4381 & 860 & 170 & 331 & 23.8 & \begin{tabular}{l|l}
13.5 \\
\end{tabular} & 0.56 & 1390 & 41 & 1480 & 5 & 5.6 & 13.4 & & & 47 & 2.76 & 7.18 & 0.02 \\
\hline SA23 & VA-73 & Jemez/Soda Dam Hot Spring & 47.0 & 6.30 & & 6700 & 4539 & 4584 & 840 & 186 & 346 & 24.6 & 13.7 & 1.2 & 1500 & 36.7 & 1570 & 3.6 & 5.6 & 8.5 & & & $\frac{78}{48}$ & 5.52 & 6.59 & 0.92 \\
\hline SA23 & VA.9 & Jemez/Soda Dam Hot Spring & 48.0 & 6.40 & & 7050 & 4620 & 4616 & 938 & 183 & 340 & 24.4 & 13.2 & 1.5 & 1510 & 38.4 & 1500 & 3.67 & & \begin{tabular}{|l|l|}
0.5 \\
13.8
\end{tabular} & & & $\frac{40}{50}$ & \begin{tabular}{|l}
1.24 \\
1.24
\end{tabular} & $\frac{0.05}{5.83}$ & 0.09 \\
\hline SA25 & $\bar{A}$ & Jemez/Spence Hot Spring & 42.0 & & 8.09 & & 297 & 312 & 58.4 & 1.6 & 5.6 & 1.7 & & & 142.8 & 24 & 9.6 & 0.54 & & 0.1 & & & \begin{tabular}{|l|}
67.4 \\
\end{tabular} & 2.26 & & 4.96 \\
\hline SA25 & VA-1 & Jemez/Spence Hot Spring & 45.0 & 6.70 & & & 294 & 294 & 50 & 1.3 & 5.5 & 1.9 & 0.66 & 0.03 & 144 & 16 & 8 & 0.55 & & $\mid$ & & & \begin{tabular}{|l|}
66 \\
66 \\
\end{tabular} & 3.75 & & 0.03 \\
\hline SA25 & VA-120 & Jemez/Spence Hot Spring & 42.3 & 7.60 & & 293 & 292 & 292 & 50 & 1.4 & 5.9 & 1.57 & 0.58 & 0.08 & 140 & 17.1 & 8.2 & 0.76 & 0.1 & \begin{tabular}{|l|}
0.12 \\
\end{tabular} & & & 66 & 3.61 & 7.18 & 0.07 \\
\hline SA25 & VA-68 & Jemez/Spence Hot Spring & 42.0 & 7.01 & & 280 & 297 & 297 & 52 & 1.6 & 7.2 & 1.87 & 0.71 & 0.02 & 135 & 18 & 11 & 0.59 & & \begin{tabular}{|l|l|}
0.15 \\
\end{tabular} & & & 69 & 0.17 & 4.24 & 0.05 \\
\hline SA25 & VA-72 & Jemez/Spence Hot Spring & 42.0 & 7.45 & & 275 & 276 & 276 & 44 & 1.4 & 6.4 & 1.76 & 0.74 & 0.04 & 138 & 2.8 & 11.2 & 0.69 & & 0.17 & & & 69 & 2.90 & 8.31 & 0.07 \\
\hline SA27 & $88 T 0110$ & Jemez Pueblo Indian Hot Spring & 36.0 & 6.91 & 6.70 & & 3176 & 4300 & 1149 & 50.2 & 96.5 & 13.1 & 4 & 2.48 & 1300 & 295.1 & 1328.5 & 6.68 & & 6.58 & & & 55.8 & 6.39 & & 35.39 \\
\hline SA27 & 88TD19 & Jemez Pueblo Indlan Hot Spring & 43.0 & 6.90 & 6.73 & & 3572 & 4082 & 983 & 51.3 & 75.4 & 11.1 & 4.4 & 2.29 & 1411.9 & 308.8 & 1165.9 & 7.5 & & \begin{tabular}{|l|}
7.49 \\
\end{tabular} & & & \begin{tabular}{|l|}
52.6 \\
\end{tabular} & 11.95 & & 14.27 \\
\hline SA28 & 287 & Jemez/McCauley Hot Spring & 30.0 & 8 & & 140 & & 197 & 19 & 0.9 & 8.7 & 4.7 & & & 94 & 6.6 & 3.8 & 1 & & & 0.02 & 1.86 & 56 & 4.78 & 19.30 & \\
\hline SA28 & 289 & Jemez/McCauley Hot Spring & 31.0 & & & 165 & & 224 & 25 & 1.4 & 12 & 4.8 & 0.27 & & 88 & 6.8 & 28 & 0.9 & 0.1 & & 0.02 & \begin{tabular}{|l|}
2.21 \\
\end{tabular} & 54 & 5.82 & 30.66 & \\
\hline
\end{tabular}




\begin{tabular}{|c|c|c|c|c|c|c|c|c|c|c|c|c|c|c|c|c|c|c|c|c|c|c|c|c|c|c|}
\hline TTEID & SAMPLE & NAME & \begin{tabular}{|c|} 
TMP \\
$c$ \\
\end{tabular} & \begin{tabular}{|c|}
$\rho H$ \\
freld \\
\end{tabular} & \begin{tabular}{|c|}
$p H$ \\
lab \\
\end{tabular} & $\begin{array}{l}C O N D \\
u S / c m \\
\end{array}$ & \begin{tabular}{|c} 
TDS \\
mgl \\
\end{tabular} & $\begin{array}{c}\text { TDS } \\
\text { (sum) }\end{array}$ & $\begin{array}{c}\mathrm{Na} \\
m g / L \\
\end{array}$ & $\begin{array}{c}K \\
q \mathcal{L} \\
\end{array}$ & $\begin{array}{c}\mathrm{Ca} \\
\mathrm{mg} / \mathrm{L}\end{array}$ & \begin{tabular}{|c|}
$M g$ \\
$m g / L$
\end{tabular} & \begin{tabular}{|c|} 
\\
moll \\
\end{tabular} & $\begin{array}{c}S r \\
m g / L\end{array}$ & $\begin{array}{l}\mathrm{HCOS} \\
\text { moll }\end{array}$ & $\begin{array}{c}504 \\
\mathrm{mo} / \mathrm{L}\end{array}$ & $\begin{array}{c}c \\
m g / h\end{array}$ & $\begin{array}{c}F \\
\text { mol }\end{array}$ & $\begin{array}{l}B r \\
d L\end{array}$ & $\begin{array}{c} \\
\mathbf{m} / L \\
\end{array}$ & $=\begin{array}{c}F e \\
m g / 2\end{array}$ & \begin{tabular}{|l|}
$\mathrm{NO3}$ \\
$\mathrm{mgl}$ \\
\end{tabular} & & $\begin{array}{l}\text { CHRG } \\
\text { BAL \& }\end{array}$ & $\begin{array}{l}\text { COND } \\
\text { BAL } 6 \\
\end{array}$ & $\begin{array}{l}\text { MASS } \\
\text { BAL\% }\end{array}$ \\
\hline \begin{tabular}{|l|} 
SA28 \\
\end{tabular} & $B$ & Jemez/McCauley Hot Spring & 32.0 & & 7.67 & & 220 & 195 & 20.9 & 0.8 & 8.4 & 4.4 & & & 81.7 & 19 & 2.1 & 0.86 & & & & & & & & $\frac{11.42}{11.42}$ \\
\hline \begin{tabular}{|l|} 
SA2 \\
\end{tabular} & $\frac{1}{V A-119}$ & Jemez/McCauley Hot Spring & \begin{tabular}{|l|}
31.9 \\
\end{tabular} & & & 173 & 186 & 186 & 20 & $\frac{0.0}{1}$ & 8.5 & $\frac{7.4}{4.32}$ & 0.22 & 0.04 & 80.6 & 5.8 & $\frac{.1}{4.4}$ & 0.05 & & 0.01 & & & $\frac{30.14}{54}$ & \begin{tabular}{|l|}
-3.02 \\
0.19 \\
\end{tabular} & 1.29 & 77.42 \\
\hline \begin{tabular}{|l|} 
SA28 \\
\end{tabular} & VA.3 & Jemez/MCCauley Hol Spring & \begin{tabular}{|l|l|}
31.0 \\
\end{tabular} & 6.20 & & & 189 & 268 & 18 & 0.8 & 8.5 & $\frac{4.32}{4.9}$ & 0.24 & \begin{tabular}{|l|l|}
0.02 \\
\end{tabular} & $\frac{80.0}{86}$ & 86 & $\frac{4.4}{6}$ & 0.05 & & 0.24 & & & $\frac{34}{56}$ & \begin{tabular}{|l|}
0.19 \\
34.42 \\
\end{tabular} & 1.29 & $\begin{array}{ll}0.09 \\
4.56\end{array}$ \\
\hline \begin{tabular}{|l|} 
SA29 \\
\end{tabular} & 424 & Jemez/San Antonio Hot Springs & \begin{tabular}{|l|}
40.0 \\
\end{tabular} & 7.7 & & 910 & & 181 & 23 & $\frac{0.0}{2}$ & 4.7 & 0.3 & $0 .<4$ & $0.0 \leq$ & $\frac{\infty}{61}$ & $\frac{60}{8.8}$ & $\frac{0}{2.3}$ & 0.8 & & $\frac{0.24}{0.04}$ & & 1.64 & 30 & \begin{tabular}{|l|}
34.42 \\
0.77 \\
\end{tabular} & 19.17 & \\
\hline \begin{tabular}{|l|} 
SA29 \\
\end{tabular} & $\mathrm{F}$ & Jemez/San Antonio Hot Spring & 56.0 & & 8.02 & & 148 & 180 & 23.2 & 2 & 3.2 & 0.5 & & & $\frac{01}{59.8}$ & 0.0 & $\frac{2.3}{5}$ & 0.8 & & $\frac{0.04}{0.02}$ & & 7.64 & $\frac{16}{85.8}$ & $\frac{0.71}{4.04}$ & 19.16 & 21. \\
\hline \begin{tabular}{|l|} 
SA29 \\
\end{tabular} & VA-12B & Jemez/San Antonio Hot Spring & & 7.88 & & 127 & 167 & 177 & $\frac{2.6}{23}$ & \begin{tabular}{|c|} 
\\
.8 \\
\end{tabular} & $\frac{3.2}{3}$ & 0.5 & .06 & 0.06 & 57.3 & 9.5 & $\frac{5}{7}$ & 0.76 & & 0.02 & & & $\frac{8.28}{74}$ & $\frac{.04}{.85}$ & & 21. \\
\hline \begin{tabular}{|l|} 
SA29 \\
\end{tabular} & $V A-4$ & Jemez/San Antonio Hot Spring & & & & $\frac{121}{150}$ & 170 & 170 & $\frac{-21}{21}$ & 1.0 & 2.3 & 0.3 & .0. & \begin{tabular}{|l|l|}
0.02 \\
\end{tabular} & $\frac{31.3}{56}$ & $\frac{9.5}{7}$ & $\frac{1}{2}$ & $\frac{0.16}{0.8}$ & & & & & 14 & 80 & & \\
\hline \begin{tabular}{|l|} 
SA29 \\
\end{tabular} & VA.96 & Jemez/San Antonio Hot Spring & \begin{tabular}{|l|}
40.8 \\
\end{tabular} & & & $\frac{150}{140}$ & 168 & 168 & $\frac{-61}{21}$ & 1.8 & 2.7 & \begin{tabular}{|l|}
0.28 \\
\end{tabular} & 0.12 & \begin{tabular}{|l|l|}
0.02 \\
02
\end{tabular} & 50 & $\frac{1}{7.6}$ & $\frac{2}{2.2}$ & $\frac{0.8}{2.2}$ & & & & & & & & \\
\hline \begin{tabular}{|l|} 
SA32 \\
\end{tabular} & VA.5 & Jemez/Soda Dam Grotto Spring & 38.0 & & & & 3950 & 3948 & $\frac{11}{1000}$ & \begin{tabular}{|l|l}
100 \\
174 \\
\end{tabular} & 324 & $\frac{0.20}{27}$ & $\frac{0.12}{13.2}$ & \begin{tabular}{|l|l|}
1.42 \\
\end{tabular} & $\frac{20}{834}$ & $\frac{1.6}{41}$ & $\frac{2.2}{1480}$ & $\frac{2.2}{4}$ & & 1.8 & & & & $\frac{90}{45}$ & & \\
\hline \begin{tabular}{|l|} 
SA33 \\
\end{tabular} & VA-94 & Jemez/Bathhouse HS & \begin{tabular}{|l|l|}
37.4 \\
\end{tabular} & 7.72 & & 178 & $\frac{2001}{261}$ & $\frac{224}{224}$ & 27 & $\frac{1.14}{4.04}$ & 5.3 & $\frac{21}{0.4}$ & $\frac{1.2}{0.09}$ & \begin{tabular}{|l|}
1.4 \\
0.04 \\
\end{tabular} & $\frac{054}{61}$ & $\frac{41}{12.7}$ & $\frac{7480}{8.6}$ & $\frac{4}{1.84}$ & & 71.8 & & & $\frac{38}{103}$ & $\begin{array}{lll}45 & -1\end{array}$ & & \\
\hline \begin{tabular}{|l|l|} 
SA33 \\
\end{tabular} & VA-126 & Jemez/Bathhouse HS & 38.1 & 7.61 & & 166 & 498 & 243 & 27 & 4.2 & $\frac{3.2}{6}$ & 0.4 & 0.08 & 0.09 & 75.6 & $\frac{12.1}{15.2}$ & 6.0 & 1.46 & 0.2 & & & & & & & \\
\hline & VA-20 & Jemez/Bathhouse HS & 38.0 & 6.10 & & 163 & 220 & 226 & $\frac{1.5}{24.5}$ & 3.6 & 4.98 & 0.4 & 0.00 & 0.02 & 71 & $\frac{10.6}{15}$ & $\frac{0.5}{2.4}$ & 1.40 & & & & & 96 & & & \\
\hline & VA-2 & Jemez几ittle Spence Hot Spring & 34.0 & 6.70 & & & 321 & 321 & $\frac{27.0}{56}$ & $\frac{3.5}{1.5}$ & 8.8 & 1.9 & 0.66 & \begin{tabular}{|l|l|}
0.04 \\
0.04
\end{tabular} & $\frac{1}{152}$ & $\frac{15}{25}$ & $\frac{.44}{7}$ & 1.0 & & 0.13 & & & $\frac{90}{67}$ & 25 & & \\
\hline & 436 & Star Lake \#2 Ojo Encino Well & 33.0 & 8.2 & & 3000 & 2190 & 2407 & 710 & 5.4 & $\frac{0.0}{26}$ & 5.8 & $\frac{0.00}{0.2}$ & 1.6 & $\frac{104}{223}$ & $\frac{20}{1300}$ & $\frac{1}{120}$ & & & & 1.02 & 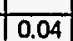 & of & & 9.54 & \\
\hline 37 & VA-110 & mez/Soda Dam Hidden Warm Spring & 32.3 & 3.13 & & 6150 & 3930 & 3934 & 817 & 130 & 226 & 15.3 & 10.5 & $\mid \frac{1.34}{134}>3$ & $\frac{2324}{1324}$ & 53 & 1294 & 3.21 & & $\frac{0.00}{13.4}$ & & & 14 & & & \\
\hline 37 & VA-141 & mez/Soda Dam Hidden Warm Spring & 32.2 & 6.42 & & 6260 & 4020 & 4018 & 780 & 125 & 305 & 20 & \begin{tabular}{|l|l|}
11.1 \\
\end{tabular} & 2.55 & $\frac{1024}{1425}$ & $\frac{50}{49}$ & $\frac{1294}{1240}$ & 蕰.04 & 3.8 & 0.6 & & & 43 & & & \\
\hline 37 & VA-27 & mez/Soda Dam Hidden Warm Spring & 29.0 & 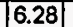 & & 5700 & 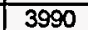 & & 720 & 141 & 376 & 18.8 & 10.8 & 1.9 & 1400 & 69.1 & 1195 & 2.71 & & & & & 国 & & & \\
\hline & 228 & & 32.0 & $\begin{array}{ll}9.5 \\
\end{array}$ & & 3140 & 2460 & 1274 & 740 & & 1.2 & 0.3 & & & 50 & 49 & 97 & $\frac{3.4}{3.4}$ & & $\frac{5}{2}$ & 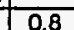 & & $\frac{10}{24}$ & & & \\
\hline SA39 & $88 \mathrm{TD} / 4$ & Penasco \#3 Spring & 27.0 & 6.79 & 6.64 & & 9672 & 10400 & 2371 & 98.5 & 396.4 $>>>>3$ & 51.1 & 5.25 & 6.63 & $\frac{1592.5}{1592}$ & $\frac{75}{3438}$ & 2405.8 & 3.59 & & $\frac{.1 .4}{7.9}$ & & & 24 & & & \\
\hline \begin{tabular}{|l|} 
SA40 \\
\end{tabular} & $88 \mathrm{TD} 16$ & Penasco \#4 Spring & 27.0 & 6.56 & 6.54 & & 9924 & 10741 & 2554 & 98.1 & 386 & 48.8 & 5.45 & 6.6 & 1876.5 & 3430 & & 3.53 & & 7.9 & & & 22 & & & $\frac{04}{23}$ \\
\hline \begin{tabular}{|l} 
SA41 \\
\end{tabular} & $88 \mathrm{TD} 18$ & Salado Warm Spring & 25.0 & 6.79 & 6.54 & & 9608 & 10742 & 2463 & 82.8 & 352.2 & 52.9 & 4.4 & 7.68 & \begin{tabular}{|l|l|}
2174.6 \\
\end{tabular} & 3215 & 2365.5 & 3.9 & & $\because$ & & & $\frac{24}{13}$ & 1.44 & & 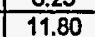 \\
\hline \begin{tabular}{|l|l} 
SA41 \\
\end{tabular} & M77.SA2 & Salado Warm Spring & 25.0 & 6.25 & & & 10465 & 10432 & 3000 & 91 & 390 & $\frac{25}{65}$ & 5.2 & & 1855 & 2600 & 2400 & $\frac{4}{4}$ & & 5.9 & & & 15 & & & \\
\hline \begin{tabular}{|l|l} 
SA42 \\
\end{tabular} & $V A-130$ & San Ysidro Warm Spring & 22.0 & 6.97 & & 9400 & 6810 & 6814 & 1720 & 77 & 305 & 71.3 & 8.1 & 4.62 & 1740 & 1183 & 1671 & 3.32 & 5 & 77 & & & $\frac{16}{16.3}$ & & 5.47 & \\
\hline \begin{tabular}{|l} 
SA42 \\
\end{tabular} & VA-33 & San Ysidro Warm Spring & 27.0 & 6.57 & & 11550 & 7170 & 7173 & 1710 & 75.3 & 375 & 128 & 5.3 & 7.7 & & 1165 & 1820 & 3.95 & & 8.32 & & & $\frac{1.0}{14}$ & & $\frac{0.41}{7.80}$ & \\
\hline SA43 & 88TD13 & Swimming Pool Spring & 24.0 & 7.27 & 7.21 & & 7420 & 8305 & 2066 & 57.8 & 267 & 48.1 & 3.25 & 6.64 & 1545 & 1896 & 2373.6 & 3.5 & & $\frac{4.95}{4.95}$ & & & 14 & 9.41 & & $\frac{0.04}{11.92}$ \\
\hline \begin{tabular}{|l|l} 
SA44 \\
\end{tabular} & PC1-1 & Jemez/PC-1 at $1712 \pi$ (w/drilling nuld) & & 7.21 & & 9500 & 7510 & 7508 & 1390 & 153 & 762 & 52.8 & 10.8 & 3.52 & 1133 & 2157 & 1602 & 1.06 & 4.3 & 3.4 & & & $\frac{2625}{225}$ & & .09 & \\
\hline$\widehat{S A 44}$ & PC2-6 & Jemez/PC-2 at $1335 \pi$ (w/drilling fiuld) & 40.0 & 7.17 & & 4980 & 5577 & 2863 & 670 & 69 & 646 & 215 & 1.86 & 3.46 & 1102 & 28.7 & 57 & 1.34 & & 2.3 & & & 66 & & & $\frac{0.05}{48.67}$ \\
\hline$S F 2$ & 362 & Los Alamos \#1B Well & 31.0 & 7.7 & & 766 & & 726 & 182 & 4.1 & 10 & 0.5 & & & 428 & 43 & 16 & 2.5 & & & & 1.1 & 39 & 40 & 1.78 & \\
\hline $\mathrm{SF} 2$ & 363 & Los Alamos \#1B Well & 31.0 & 7.7 & & 766 & & 726 & 180 & 4 & 10 & 0.5 & & & 430 & 43 & 16 & 2.5 & & & & 1.7 & 39 & & & \\
\hline 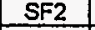 & 364 & Los Alamos \#1B Well & 30.5 & 7.8 & & 729 & & 684 & 170 & 3.9 & 9.8 & 0.4 & & & 399 & $\frac{42}{42}+2>2>3$ & 16 & $2.5>>>>3$ & & & & 1.2 & 39 & & & \\
\hline SF2 & 365 & Los Alamos \#1B Well & 30.5 & 7.8 & & 729 & & 685 & 170 & 4 & 9.8 & 0.4 & & & 400 & 42 & 16 & 2.5 & & & & 1.2 & 39 & & 10.00 & \\
\hline SF2 & 366 & Los Alamos \#1B Well & 30.5 & 7.8 & & 720 & & 680 & 169 & 3.9 & 9.4 & 0.6 & & & 394 & 41 & 16 & 2.5 & & & & 1.2 & 42 & 48 & 10.69 & \\
\hline SF2 & LA-7 & Los Ala & 30.0 & 7.20 & & & 559 & 559 & 138 & 2 & 6.5 & 0.3 & 0.11 & 0.14 & 326 & 32 & 15 & 2.3 & & 0.45 & & & 36 & & & 0.04 \\
\hline SF3 & LA-12 & G6 Well & 30.5 & 6.50 & & & 191 & 191 & 15 & & 15 & 2.3 & & 0.06 & 94 & 5 & 2 & 0.27 & & & & & 55 & & & \\
\hline SF5 & LA.16 & S \#G2 Well & 30.0 & 6.50 & & & 256 & 256 & 33 & 2.5 & 11. & 0.61 & 0.02 & 0.08 & 12 & 5 & 4 & 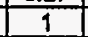 & & 0.12 & & & 77 & & & \\
\hline $\mathrm{S} 12$ & SNM-3 & TORC A & 41.2 & 7.00 & 7.34 & 5140 & 2697.5 & 2696 & 751 & 56 & 163 & 15.3 & 1.3 & 3.82 & & 75. & & 3.06 & 2.6 & & & & 45 & & 14.85 & \\
\hline$s 13$ & 89 & Torc Blackst & \begin{tabular}{|l|}
45.0 \\
\end{tabular} & & 7.79 & & 2608 & 2736 & 817.5 & 61.4 & 143.9 & \begin{tabular}{|l|l|}
18.0 \\
\end{tabular} & \begin{tabular}{|l|}
1.21 \\
\end{tabular} & \begin{tabular}{|l|}
.0 .12 \\
\end{tabular} & 164.7 & 96 & 1285.2 & 1.49 & 0.77 & 0.38 & & & 44.3 & $\frac{24}{29}$ & & \\
\hline $\mathrm{SI6}$ & $\overline{S 76 T R C 14 A}$ & TorC Yuccal & 42.8 & & 7.30 & 4510 & 2670 & & 735 & 61 & 154 & 21 & & & 216 & 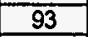 & 1290 & 3.8 & & & & & 41 & & & 2.07 \\
\hline $\mathrm{SI6}$ & S76TRC14B & Tore Yucca Lodge well $14 \mathrm{ft}$ & \begin{tabular}{|l|}
40.0 \\
\end{tabular} & 6.70 & & \begin{tabular}{|l|}
4500 \\
\end{tabular} & & $\frac{20}{27}$ & 742 & \begin{tabular}{|l|l|}
57 \\
\end{tabular} & 175 & \begin{tabular}{|l|}
18.8 \\
\end{tabular} & 1.5 & & 250 & $\frac{50}{120}$ & $\frac{12}{13}$ & 0.0 & & 0.2 & & & \begin{tabular}{|l|l|}
41 \\
\end{tabular} & & $\frac{4.108}{1.71}$ & \\
\hline 些 & 810 & TorC Siema Mineral Bath & \begin{tabular}{|l|}
41.0 \\
4
\end{tabular} & & 7.80 & & & 2755 & 791.5 & \begin{tabular}{|l|l|}
63 \\
63
\end{tabular} & \begin{tabular}{|l|}
143.9 \\
\end{tabular} & \begin{tabular}{|l|l|}
17.9 \\
\end{tabular} & \begin{tabular}{|l|}
1.23 \\
\end{tabular} & .12 & $\frac{200}{162.3}$ & 120 & & 3.1 & & & & & \begin{tabular}{|l|}
44.3 \\
41
\end{tabular} & 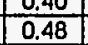 & & \\
\hline & $\pi$ & & \begin{tabular}{|l|}
41.0 \\
\end{tabular} & & 7.88 & & & 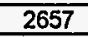 & 764.6 & & & 17.1 & 1.24 & & 136.7 & & & 3.1 & & & & & & & & \\
\hline S11 & B15 & TorC & 41.0 & & 7.98 & & & 27 & 785 & 62.6 & & 18.7 & 1.2 & 4.08 & 22 & & & 3.2 & 0.82 & 0.38 & & & & & & 0.36 \\
\hline S|1 & GS2094S & Torc old G & & & & 4520 & 2560 & 25 & & 39 & 1 & 14 & & & & & & 帮 & & & & & 36 & 0.44 & 7.89 & \\
\hline SI1 & 10 & & & & & & & & 7. & $\frac{3}{3}$ & 15 & 1 & & & 22 & & & & & & & 10 & & & & \\
\hline & 11 & & & & & & & 24 & & 2 & & 1 & & & & & & & & & & & & & 93 & \\
\hline SI1: & 98 & 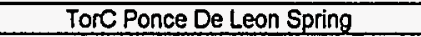 & 39.0 & & & 4400 & 244 & 24 & 7 & & & & & & $\overline{22}+2>>+2>$ & & & & & & & & & & & \\
\hline S11 & 106 & To & 38.5 & & & & & 24 & & & & & & & 21 & & & & & & 0. & 5 & 31 & & & \\
\hline 5 & GS2 & TorC $G$ & & & & $42>>2>2>>3$ & 241 & 2 & 67 & 3 & & & & & 212 & & 14 & & & & & & 37 & 0.43 & 8.82 & $\overline{0.12}$ \\
\hline $\mid \mathbf{S}$ & SNN & TorCG & 42,0 & 7.00 & 7.47 & 5120 & & 20 & 750 & 58 & 109 & 14.6 & 1.3 & 4.04 & 212 & 74 & 13 & 3.2 & 2.6 & & & & 46 & 0 & 14.99 & \\
\hline $\mathrm{SI}$ & JUS & Spring & 34,5 & & & & 5 & 77 & 168.5 & 10.9 & 5.4 & 0.4 & & & 275.8 & 129 & 21 & 14.8 & & & & & 152 & 4 & & 36.97 \\
\hline Sl & 85 & Springs & & & 8.23 & & 1240 & 1339 & 323.9 & 18.8 & 47.1 & 15.8 & 0.36 & \begin{tabular}{|l|}
0.86 \\
\end{tabular} & 366.1 & 376.6 & 15 & 5.9 & f & 0.34 & & & 31.3 & 0 & & 7.98 \\
\hline SI1 & 86 & Derry Warm Springs & & & 8.62 & & 1228 & 133 & 340 & 19.2 & 47.1 & 16 & 0.35 & 0.83 & 311.2 & 374.6 & 153.2 & 5.9 & to & 0.37 & & & 32.3 & 4.0 & & 6.02 \\
\hline SI19 & GS1510 & Derry Warm Springs & & .20 & & 1660 & & 123 & 300 & 18 & 45 & 15 & 0.31 & & 355 & 290 & 170 & 7.3 & 0.5 & 0.33 & & & 33 & 0.02 & 2.62 & \\
\hline
\end{tabular}




\begin{tabular}{|c|c|c|c|c|c|c|c|c|c|c|c|c|c|c|c|c|c|c|c|c|c|c|c|c|c|c|}
\hline SITE ID & SAMPLE & NAME & $\begin{array}{c}T M P \\
C \\
\end{array}$ & \begin{tabular}{|l|}
$\mathrm{pH}$ \\
field \\
\end{tabular} & \begin{tabular}{l|}
$\mathrm{pH}$ \\
$\mathrm{lab}$ \\
\end{tabular} & $\begin{array}{l}\text { COND } \\
\mathrm{uS} / \mathrm{cm}\end{array}$ & $\begin{array}{c}\text { TDS } \\
\text { moll }\end{array}$ & $\begin{array}{c}\text { TDS } \\
\text { (sum) }\end{array}$ & $\begin{array}{c}\mathrm{Na} \\
\mathrm{mol}\end{array}$ & $\begin{array}{c}K \\
m \sigma / h\end{array}$ & \begin{tabular}{c|}
$\mathrm{Ca}$ \\
$\mathrm{mg/L}$
\end{tabular} & \begin{tabular}{c|}
$M g$ \\
$m g / L$
\end{tabular} & $\begin{array}{c} \\
m g / L\end{array}$ & \begin{tabular}{c|}
$S r$ \\
$m g h$
\end{tabular} & \begin{tabular}{|l|}
$\mathrm{HCO} 3$ \\
$\mathrm{mg} / \mathrm{L}$
\end{tabular} & $\begin{array}{c}\mathrm{SO4} \\
\mathrm{mg} / \mathrm{L}\end{array}$ & $\begin{array}{c}c \\
m g / 2\end{array}$ & \begin{tabular}{|c|}
$F$ \\
$m q / L$ \\
\end{tabular} & $\begin{array}{l}B r \\
g / L\end{array}$ & \begin{tabular}{|c|}
$B$ \\
$m \mathscr{L} / L$ \\
\end{tabular} & \begin{tabular}{|c|}
$F e$ \\
$m p / 2$
\end{tabular} & \begin{tabular}{|l|}
$\mathrm{NO}^{2}$ \\
$\mathrm{mg} / \mathrm{L}$
\end{tabular} & \begin{tabular}{|c|} 
SIO2 \\
$\sigma / 2$
\end{tabular} & \begin{tabular}{|l|} 
CHRG \\
BAL \%
\end{tabular} & $\begin{array}{l}\text { COND } \\
\text { BAL \& }\end{array}$ & $\begin{array}{l}\text { MASS } \\
\text { BAL } \%\end{array}$ \\
\hline S119 & S76DWS-65 & Derry Warm Springs & 33.8 & & 8.39 & 1420 & 823 & 1121 & 303 & 17 & 32.8 & 12.9 & 0.32 & & 295 & 257 & 162 & 3.67 & 1.42 & 0.38 & & & 36 & 4.44 & 15.21 & 36.27 \\
\hline S119 & S76DWS-74 & Derry Warm Springs & 33.8 & & & & & 1311 & 340 & 17 & 40 & 15 & \begin{tabular}{|l|}
0.4 \\
\end{tabular} & & 397 & 309 & 155 & $\frac{3.01}{6}$ & $\mid$\begin{tabular}{|l|}
0.21 \\
0.21 \\
\end{tabular} & 0.6 & & & $\frac{\pi}{31}$ & $\frac{7.47}{2.44}$ & & \\
\hline$S 121$ & 92 & well & 34.0 & 7.2 & & 1660 & & 1235 & 300 & 18 & 45 & 15 & 0.31 & & 355 & 290 & 170 & 7.3 & 0.5 & 0.33 & & 0.49 & 33 & 0.02 & 2.62 & \\
\hline S122 & 83 & well & 31.0 & 8.3 & & 1350 & 953 & 985 & 280 & 5.1 & 5.9 & 0.2 & & & 179 & 430 & 30 & 5.3 & & 1.3 & 0.25 & 4.87 & 51 & 1.02 & 6.51 & 3.35 \\
\hline S123 & $\mathrm{B} 12$ & warm spring & & & 7.90 & & 1392 & 1597 & 387.4 & 21.5 & 110.4 & 9.5 & 0.42 & 2.21 & 211.1 & 211.1 & 602.7 & 2.46 & 0.78 & \begin{tabular}{|l|}
0.2 \\
\end{tabular} & & & 37.3 & 2.45 & & 14.73 \\
\hline$S 123$ & \begin{tabular}{|l|} 
S7GAFTER \\
\end{tabular} & Barney lorio \#1 Fee & 33.0 & & 7.38 & 5600 & 4931 & 4694 & 1280 & 7.8 & 375 & 0.46 & \begin{tabular}{|l|}
0.91 \\
\end{tabular} & & 17 & 2721 & 262 & 3.5 & \begin{tabular}{|l|}
1.72 \\
\end{tabular} & 0.49 & & & \begin{tabular}{|l|}
23.9 \\
\end{tabular} & 7.35 & 33.50 & 4.81 \\
\hline S.J1 & 440 & well & 62.0 & 7.8 & & 11400 & & 10933 & 3600 & 22 & 71 & 14 & & 4.1 & 232 & 6400 & 550 & 3.6 & & 1.5 & 0.03 & 0.04 & 35 & 2.92 & 42.06 & \\
\hline $5 \sqrt{2}$ & 441 & well & 57.0 & & & 4350 & & 3526 & 770 & 8 & 340 & 5 & & & 57 & 2400 & 14 & 2.3 & & & \begin{tabular}{|l|}
0.03 \\
\end{tabular} & 0.04 & 30 & 0.33 & 17.42 & \\
\hline S.2 2 & 442 & well & 48.0 & 8 & & 4000 & 3620 & 3404 & 760 & 9.1 & 320 & 4 & \begin{tabular}{|l|}
0.27 \\
\end{tabular} & & 60 & 2200 & 19 & 2.8 & & 0.18 & & 0.04 & 29 & 2.22 & 24.07 & 5.96 \\
\hline $2 \sqrt{3}$ & 446 & Navajo well & 61.0 & 8.1 & & 1390 & 880 & 1009 & 250 & 2.5 & $\frac{39}{39}$ & 0.5 & & & 166 & 490 & $\frac{10}{17}$ & $\frac{2.0}{1}$ & & & & 0.13 & 43 & 2.00 & 7.01 & 14.67 \\
\hline S. 4 & 443 & Dome Well Chaco & 42.0 & 8.3 & & 10000 & & 8611 & 2800 & 15 & 70 & 8.3 & & & 570 & 4300 & 810 & 5.3 & & 1.9 & & \begin{tabular}{|l|}
0.18 \\
\end{tabular} & 30 & 1.76 & 26.36 & \\
\hline 518 & 437 & well & 32.8 & 8.2 & 8.3 & 2720 & & 1998 & 630 & 3.3 & 11 & 2.1 & 0.1 & 0.35 & 375 & 910 & 47 & 1.6 & 0.18 & & 03 & & 17 & 316 & 380 & \\
\hline S. 18 & 439 & well & 32.9 & 8.3 & 8.4 & 2800 & & 2184 & 670 & 3.2 & 7.4 & 2.4 & 0.12 & \begin{tabular}{|l|}
0.35 \\
\end{tabular} & 334 & 1100 & 49 & 1.6 & 0.06 & & 0.25 & & $\frac{16}{16}$ & 0.04 & 6.50 & \\
\hline SM3 & NM1 & Montezuma Hot Spring & 53.8 & & 9.09 & & 432 & 589 & 180.5 & 5.9 & 4.8 & 0.1 & 0.44 & 0.03 & 69.5 & 80.7 & 153.1 & 20.7 & \begin{tabular}{|l|}
0.58 \\
\end{tabular} & 0.47 & & & 72.5 & 0.48 & & 36.42 \\
\hline SM3 & NM2 & Montezuma Hot Spring & 48.0 & & 9.10 & & 452 & 566 & 180.5 & 5.9 & 3.6 & 0.1 & & & 72 & 79.7 & 151 & 0.67 & & 0.5 & & & \begin{tabular}{|l|}
72.5 \\
\end{tabular} & 6.89 & & 25.33 \\
\hline SM3 & NM3 & Montezuma Hot Spring & 34.3 & & 8.16 & & 464 & 606 & 173.8 & 5.9 & 5.8 & 0.2 & & & 120.8 & 80.7 & 148.9 & 0.64 & & 0.56 & & & 69 & 0.77 & & 30.67 \\
\hline SM3 & NM4 & Montezuma Hot Spring & 53.0 & & 9.01 & & 460 & 578 & 185.3 & 5.9 & 3.6 & 0.1 & & & 78.1 & 80.7 & 152.1 & 0.66 & & 0.7 & & & 71 & 7.10 & & 25.69 \\
\hline SM3 & NM5 & Montezuma Hot Spring & 58.5 & & 9.03 & & 448 & 579 & 186.2 & 5.9 & 3.2 & 0.1 & 0.44 & 0.02 & 73.2 & 78.8 & 153.9 & 0.67 & 0.8 & 0.67 & & & 75 & 7.99 & & 29.22 \\
\hline SM3 & NM6 & Montezuma Hot Spring & 35.6 & & 9.10 & & 400 & 572 & 184.1 & 5.9 & 3.2 & 0.1 & & & 73.2 & 81.6 & 151 & 0.67 & & 0.5 & & & 72 & 7.30 & & 43.07 \\
\hline SO1 & CH823-66 & core hole & 42.2 & & 7.70 & 3460 & & 1968 & 626 & 38 & 68 & 2.1 & & & 109 & 163 & 945 & 1.1 & & 0.62 & & & 23 & 0.01 & 8.18 & \\
\hline $\mathrm{SO} 2$ & 166 & warm well & 36.0 & 8.3 & & 430 & & 356 & 82 & 1.7 & 8.4 & 1.5 & & & 124 & 50 & 24 & 3.2 & & 0.14 & 0.2 & 7.09 & 54 & 2.90 & 3.42 & \\
\hline $\mathrm{SO3}$ & 126 & Welty Salty Well & 35.0 & 7.5 & & 2100 & 1440 & 1503 & 290 & 29 & 160 & 11 & & & 120 & 580 & 280 & 3.6 & & 0.19 & 0.18 & 0.09 & 29 & 0.26 & 5.93 & 4.38 \\
\hline SO5 & 127 & warm well & 33.0 & 7.4 & & 4600 & & 2984 & 810 & 31 & 120 & 41 & & & 410 & 560 & 980 & 1 & & 0.88 & 1.3 & 4.87 & 24 & 0.75 & 1.33 & \\
\hline SO6 & 163 & Blue Canyon Well & 31.8 & 8.1 & & 380 & & 337 & 56 & 3 & 21 & $\frac{1}{4}$ & & & 170 & 39 & 13 & 0.6 & & 0.08 & \begin{tabular}{|l|}
0.05 \\
\end{tabular} & $\frac{7.01}{1.3}$ & $\frac{67}{29}$ & 1.36 & 2.36 & \\
\hline 506 & \begin{tabular}{|l|} 
BLUUE12-74 \\
\end{tabular} & Blue Canyon Well & & & 7.40 & & & 342 & 68 & 2.5 & 16 & 4.3 & 0.08 & & 167 & 40 & 16 & 0.8 & 0.54 & & & & 27 & 1.40 & & \\
\hline 506 & GS-BLUE & Blue Canyon Well & 32.2 & & 7.60 & 375 & & 324 & 56 & 3 & 20 & 4.6 & & & 163 & 36 & 14 & & & & & & 27 & 0.95 & 3.71 & \\
\hline 506 & GS54325 & Blue Canyon Well & 32.2 & & 8.10 & 380 & & 331 & 56 & 2.8 & 21 & 4 & & & 166 & 39 & 13 & 0.6 & & 0.08 & & & 29 & 0.59 & 2.22 & \\
\hline SO7 & 140 & Socorro Gallery Spring & 32.0 & 7.8 & & 348 & 224 & 251 & & 2.8 & 18 & 3.9 & & & 154 & 28 & 15 & 0.6 & & & & 1.2 & 27 & 46.80 & 62.91 & 11.83 \\
\hline $\mathrm{SO7}$ & 148 & Socorro Gallery Spring & 30.0 & 7.8 & & 348 & 224 & 297 & 52 & 2.8 & $18^{\circ}$ & 3.9 & & & 150 & 28 & 15 & 0.6 & & & & & 27 & 0.80 & 2.09 & 32.72 \\
\hline SO7 & 154 & Socorro Gallery Spring & 30.5 & 8.4 & & & & 243 & 53 & 3 & 18 & 4 & & & 130 & & 16 & 0.5 & & \begin{tabular}{|l|l|}
0.07 \\
\end{tabular} & 0.13 & & 18 & 16.10 & & \\
\hline SO7 & GS110-64 & Socorro Gallery Spring & & & 7.80 & 356 & 236 & 317 & 52 & 3 & 18 & 4.6 & & & 164 & 31 & 13 & 0.6 & & & & & 31 & 1.58 & 1.55 & 34.41 \\
\hline SO7 & JONESO4 & Socorro Gallery Spring & & & & & & 345 & 27 & 56 & 22 & 3 & & & 114 & 79 & 16 & & & & & & 28 & 0.17 & & \\
\hline SO7 & SCTTBRK & Socorro Gallery Spring & & & 7.00 & 348 & 224 & 301 & 52 & 2.8 & 18 & 3.9 & & & 154 & 28 & 15 & 0.6 & & & & & 27 & 0.13 & 2.09 & 34.51 \\
\hline SO8 & 133 & Socorro/Sedilla Gallery Spring & 30.0 & 7.9 & & 352 & 237 & 315 & 54 & 3 & 20 & 3.2 & & & 160 & 31 & 12 & 0.6 & & 0.08 & 0.07 & & 31 & 0.67 & 4.74 & 32.89 \\
\hline 508 & 137 & Socorro/Sedilla Gallery Spring & 30.5 & 7.9 & & 340 & & 288 & 51 & 3.3 & 17 & 4.4 & & & 150 & 22 & 15 & & & \begin{tabular}{|l|}
0.12 \\
\end{tabular} & & & 25 & 2.53 & 3.33 & \\
\hline 508 & 138 & Socorro/Sedilla Gallery Spring & 32.0 & 7.9 & & 352 & & 320 & 54 & 3 & 20 & 3.2 & & & 164 & 31 & 12 & 0.6 & & 0.08 & 0.07 & 1 & 31 & 0.22 & 4.74 & \\
\hline SO8 & $\mathrm{B} 13$ & Socorro/Sedilla Gallery Spring & 34.0 & & 8.48 & & 284 & 331 & 56.1 & 3.1 & 17.2 & 4.3 & 0.06 & 0.35 & 162.3 & 50 & 10.3 & 2.02 & 0.28 & 0.09 & & & 25.3 & 4.50 & & 16.69 \\
\hline 508 & GS54324 & Socorro/Sedilla Gallery Spring & 32.2 & & 7.90 & 352 & & 319 & 54 & 3 & 20 & 3.2 & & & 164 & 31 & 12 & 0.6 & & 0.08 & & & 31 & 0.22 & 4.74 & \\
\hline 508 & SNM-21 & Socorro/Sedilla Gallery Spring & 32.0 & 6.50 & 7.73 & 338 & 319.6 & 318 & 54.3 & 2.4 & 18.4 & 3.9 & 0.06 & 0.38 & 170 & 29.9 & 11.7 & 0.61 & & & & & 26 & 1.23 & 8.87 & 0.61 \\
\hline sog & 828 & well & 30.0 & & 7.84 & & 192 & 239 & 24.1 & 0.8 & 23.4 & 1.7 & & & 108.5 & 26.4 & 1.4 & 2.7 & & & & & 50.1 & 2.73 & & 24.54 \\
\hline SO11 & B25 & Monticello Box Warm Spring & 29.0 & & 8.10 & & 516 & 595 & 137.7 & 5.5 & 44.2 & 1.7 & & & 124.4 & 107.5 & 150.1 & 0.25 & & 0.02 & & & 23.6 & 0.29 & & 15.30 \\
\hline SO12 & B17 & Monticello Box Warm Spring & 28.0 & & 8.24 & & 468 & 538 & 125.5 & 5.1 & 34.9 & 1.3 & 0.11 & 0.28 & \begin{tabular}{|l|}
131.8 \\
\end{tabular} & 96.1 & 104.2 & 3.1 & 0.23 & 0.09 & & & 35.3 & 1.32 & & 14.96 \\
\hline So13 & LUC-17 & artesian well & 26.0 & 6.82 & & 4000 & & 3770 & 475 & 10.3 & 450 & 85.7 & 0.94 & 8.04 & 475 & 2070 & 145 & 2.1 & & 1.03 & 31.7 & & 15 & 4.10 & 26.87 & \\
\hline 5014 & LUC.19 & Field Artesian Well & 25.0 & 6.30 & & 3700 & & 3652 & 214 & 22 & 624 & 93.1 & 0.77 & \begin{tabular}{|l|}
9.33 \\
\end{tabular} & 376 & 2080 & 211 & 2.4 & & 0.78 & 4.7 & & 14 & 6.27 & 32.41 & \\
\hline 5015 & LUC-25 & Ojo Saladito Spring & 24.0 & 6.82 & & 12000 & & 7413 & 1990 & 46 & 355 & 123 & 1.3 & \begin{tabular}{|l|}
7.17 \\
\end{tabular} & 740 & 1170 & 2960 & 2 & \begin{tabular}{|l|l|}
9.6 \\
\end{tabular} & 1.69 & 0.11 & 3.2 & 12 & 9.78 & 3.40 & \\
\hline SP16 & B14 & Cook Spring & & & 8.33 & & 348 & 378 & 68.5 & 3.1 & 16.4 & 4.3 & 0.08 & 0.39 & 181.8 & 69.2 & 12.1 & 0.69 & 0.37 & 0,09 & & & 21.3 & 6.09 & & 8.71 \\
\hline TS1 & NM31 & Hondo Hot Spring & 40.6 & & 8.14 & & 584 & 652 & 149.2 & 12.5 & 22.8 & 5.2 & \begin{tabular}{|l|}
0.37 \\
\end{tabular} & 0.12 & \begin{tabular}{|l|}
196.5 \\
\end{tabular} & 137.4 & 57.1 & 2.49 & 0.75 & 0.23 & & & 67 & 3.68 & & 11.59 \\
\hline TS2 & 457 & Mamby Hot Spring & 38.0 & 9 & & 736 & 504 & 585 & 140 & 9.8 & 13 & 3.6 & & 0.4 & 160 & 140 & 55 & 3.1 & & 0.29 & & & 60 & 0.29 & 0.89 & 16.11 \\
\hline TS2 & 458 & Mamby Hot Spring & 34.0 & 6.9 & & 794 & & 624 & 130 & 9.2 & 27 & 5.7 & 0.29 & & 202 & 130 & 56 & 3.8 & 0.5 & 0.27 & 0.04 & 1.51 & 58 & 0.36 & 2.42 & \\
\hline TS2 & 459 & Mamby Hot Spring & 38.0 & 8.4 & & 736 & 491 & 585 & 140 & 10 & 13 & 3.6 & & & 160 & 140 & 55 & 3.1 & & 0.29 & & & 60 & 0.27 & 0.94 & 19.14 \\
\hline TS2 & GS15113 & Mamby Hot Spring & & & 6.90 & 794 & & 623 & 130 & 9.2 & 27 & 5.7 & 0.29 & & 202 & 130 & 56 & 3.8 & 0.5 & 0.27 & & & 58 & 0.36 & 2.42 & \\
\hline TS2 & NM29 & Mamby Hot Spring & 38.3 & & 7.36 & & 552 & 622 & 128 & 8.6 & 27.4 & 5.1 & 0.35 & 0.13 & 217.2 & 121 & 52.1 & 2.74 & & 0.29 & & & 59.5 & 0.42 & & 12.76 \\
\hline TS2 & NM30 & Mamby Hot Spring & 32.8 & & 7.43 & & 396 & 454 & 72.4 & 6.6 & 28.8 & 5.5 & 0.19 & 0.15 & 192.8 & 60.5 & 24.8 & 1.64 & \begin{tabular}{|l|}
0.57 \\
\end{tabular} & 0.22 & & & 60 & 0.25 & & 14.69 \\
\hline
\end{tabular}




\begin{tabular}{|c|c|c|c|c|c|c|c|c|c|c|c|c|c|c|c|c|c|c|c|c|c|c|c|c|c|c|}
\hline SITE ID & SAMPLE & NAME & $\begin{array}{c}T M P \\
C \\
\end{array}$ & \begin{tabular}{|l|}
$p H$ \\
freld \\
\end{tabular} & \begin{tabular}{|l|}
$p H$ \\
lab \\
\end{tabular} & $\begin{array}{l}\text { COND } \\
\text { uS/cm } \\
\end{array}$ & $\begin{array}{c}T D S \\
m g / L \\
\end{array}$ & $\begin{array}{c}\text { TDS } \\
\text { (sum) }\end{array}$ & $\begin{array}{c}\mathrm{Na} \\
\mathrm{mg} / \mathrm{L}\end{array}$ & $\begin{array}{c}K \\
m q / L\end{array}$ & $\begin{array}{c}\mathrm{Ca} \\
\mathrm{mg} / \mathrm{L}\end{array}$ & $\begin{array}{c}\mathrm{Mg} \\
\mathrm{mg} / \mathrm{L}\end{array}$ & \begin{tabular}{c|} 
\\
$m \alpha / L$
\end{tabular} & $\begin{array}{c}S r \\
m / L\end{array}$ & $\begin{array}{l}\mathrm{HCO} 3 \\
\mathrm{~m} \alpha / \mathrm{L}\end{array}$ & $\begin{array}{l}504 \\
m g / L\end{array}$ & $\begin{array}{c}\mathrm{Cl} \\
\mathrm{mg/L}\end{array}$ & \begin{tabular}{c|}
$F$ \\
$m g / L$
\end{tabular} & $\begin{array}{l}\mathrm{Br} \\
\mathrm{g} / \mathrm{L}\end{array}$ & \begin{tabular}{|c|} 
\\
$m o / L$
\end{tabular} \mid & \begin{tabular}{|c|}
$F_{0}$ \\
$m q / L$
\end{tabular} & \begin{tabular}{|l|} 
NO3 \\
mo/L
\end{tabular} & \begin{tabular}{|r|}
$\mathrm{S} O 22$ \\
$\mathrm{~g} / \mathrm{L}$
\end{tabular} & \begin{tabular}{|l|} 
CHRG \\
BALL \%
\end{tabular} & \begin{tabular}{|l|} 
COND \\
BAL \%
\end{tabular} & $\begin{array}{l}\text { MASS } \\
\text { BAL \% }\end{array}$ \\
\hline TS2 & S76MAM2 & Mamby Hot Spring & 37.8 & & 8.60 & 729 & 491 & 581 & 138 & 9.8 & 13 & 3.6 & 0.17 & & 160 & 138 & 55 & 3.1 & & 0.29 & & & 60 & 0.09 & 0.92 & 18.32 \\
\hline TS2 & S76MAM3 & Mamby Hot Spring & 34.4 & 6.90 & & & & 646 & 156 & 8.2 & 29 & 4.6 & & & 207 & 139 & 45 & 3.6 & 0.12 & & & & 53 & 6.48 & & \\
\hline TS3 & 460 & warm spring & 37.0 & 8 & & 760 & & 660 & 145 & 11 & 21 & 4.6 & 0.27 & & 200 & 150 & 58 & 2.9 & & 0.25 & & & 67 & 0.84 & 5.97 & \\
\hline TS4 & 451 & Rancho Del Rio Grande Well & 32.0 & 7.8 & & 786 & & 531 & 150 & 4.6 & 11 & 1.2 & 0.25 & & 79 & 120 & 92 & 18 & 0.7 & 0.5 & 0.02 & & 54 & 0.13 & 6.79 & \\
\hline TS5 & NM22 & Ponce de Leon Hot Spring & 34.4 & & 8.56 & & 512 & 589 & 160.2 & 4.3 & 10 & 0.6 & 0.31 & 0.05 & 112.3 & 144.1 & 89 & 12.6 & 0.56 & 0.55 & & & 54.5 & 2.22 & & 15.05 \\
\hline TS5 & S76SITE6 & Ponce de Leon Hot Spring & 34.0 & & 7.20 & & & 561 & 164 & 3.8 & 11 & 0.5 & 0.36 & & 104 & 118 & 96 & 12.5 & 0.32 & 0.45 & & & 50 & 2.22 & & \\
\hline$\overline{\mathrm{VA2}}$ & 172 & well & 32.5 & 9 & & 625 & 468 & 572 & 150 & 2.4 & 4.3 & 0.6 & & & 280 & 66 & 8 & 1.6 & & 0.54 & 0.21 & 0.09 & 58 & 4.40 & 9.60 & 22.17 \\
\hline
\end{tabular}




\section{APPENDIX 3}

TABLES OF PARTIAL CHEMICAL ANALYSES

NOTES:

SITE ID

SAMPLE

geothermal site number

cited or assigned sample number or designation

(see Appendix 4 for dates and data source

DATE

NAME

TEMP

month/day/yr that sample was taken or reported

well or spring name

CHEMISTRY

temperature ${ }^{\circ} \mathrm{C}$

units as shown

$\mathrm{mg} / \mathrm{L}$

uS/cm

TDS

milligrams per liter

microsiemens per centimeter

analytical total dissolved solids

$\mathrm{Na}+\mathrm{K}$

many older analyses report total sodium and potassium 


\begin{tabular}{|c|c|c|c|c|c|c|c|c|c|c|c|c|c|c|c|c|c|c|c|c|c|c|c|}
\hline SITE ID & SAMPLE & NAME & $\begin{array}{c}T M P \\
C \\
\end{array}$ & \begin{tabular}{|l|}
$p H$ \\
field \\
\end{tabular} & \begin{tabular}{|l|}
$p H$ \\
$l a b$ \\
\end{tabular} & $\begin{array}{l}\text { COND } \\
\text { uS/cm }\end{array}$ & $\begin{array}{c}\text { TDS } \\
m g / 2\end{array}$ & $\begin{array}{c}\mathrm{Na} \\
\mathrm{mg} / \mathrm{L}\end{array}$ & $\begin{array}{c}K \\
\text { mg } 2\end{array}$ & $\begin{array}{l}\mathrm{Na}+\mathrm{K} \\
\mathrm{mg} / \mathrm{L}\end{array}$ & $\begin{array}{c}\mathrm{Ca} \\
\mathrm{mg} / \mathrm{L}\end{array}$ & $\begin{array}{c}M g \\
m g / L\end{array}$ & $\begin{array}{c} \\
m g h\end{array}$ & $\begin{array}{c}s r \\
m g / 2\end{array}$ & \begin{tabular}{|l|}
$\mathrm{HCO} 3$ \\
$\mathrm{mg} /$
\end{tabular} & $\begin{array}{l}\mathrm{sO4} \\
\mathrm{mgh}\end{array}$ & $\begin{array}{c}\mathrm{Cl} \\
\mathrm{mg} / \mathrm{L}\end{array}$ & $\begin{array}{c}F \\
m g / h\end{array}$ & \begin{tabular}{c|}
$B r$ \\
$m g /$
\end{tabular} & \begin{tabular}{|c|}
$B$ \\
$m g / L$ \\
\end{tabular} & \begin{tabular}{c|}
$\mathrm{Fe}$ \\
$\mathrm{mgl}$
\end{tabular} & \begin{tabular}{|l|}
$\mathrm{NO3}$ \\
$\mathrm{mg} / \mathrm{h}$
\end{tabular} & $\begin{array}{l}\mathrm{SIO2} \\
\mathrm{mg} / \mathrm{L}\end{array}$ \\
\hline BE1 & 219 & well & 40.5 & & & 423 & & & & & & & 0.05 & & & & & & & 0.26 & & & \\
\hline BE2 & 217 & well & 34.5 & & & 402 & & & & & & & 0.05 & & & & & & & 0.23 & & & \\
\hline BE2 & JIREC-2 & West Mesa \#1 & 32.2 & & & & 348 & 114 & 1 & & 2 & 1 & & & & & & 0.96 & & & & & 23 \\
\hline $\mathrm{BE3}$ & 220 & well & 33 & 7.9 & 8.2 & 1160 & & 250 & 8.1 & & 22 & 5.1 & & & & 270 & 100 & 0.9 & & \begin{tabular}{|l|}
0.43 \\
\end{tabular} & 0.099 & & 48 \\
\hline BE3 & 221 & well & 32 & 7.8 & \begin{tabular}{|l|}
7.9 \\
\end{tabular} & 1250 & & 240 & 7.4 & & 22 & 5.2 & & & & 220 & 100 & 0.8 & & & 0.5 & 0.8 & 49 \\
\hline BE3 & 222 & well & 30.5 & 7.6 & \begin{tabular}{|l|}
8.1 \\
\end{tabular} & 1125 & & 230 & 6.2 & & 21 & 4.5 & & & & 240 & 91 & 0.8 & & & 0.083 & & 25 \\
\hline BE5 & GJIR988 & West Mesa \#4 & 32.1 & & & & & 117 & 0.8 & & 1.8 & 0.1 & & & & & & 0.83 & & & & & 38.3 \\
\hline BE5 & JIREC-4 & West Mesa \#4 & 31.7 & & & & 356 & 37 & 0.2 & & 4.7 & 1 & & & & & & 0.96 & & & & & \\
\hline BE6 & 211 & well & 32 & 8.9 & & 520 & & 120 & 1.9 & & 6.3 & 0.8 & & & & 130 & 21 & 0.9 & & & 0.03 & 7.09 & 46 \\
\hline BE7 & JIREC-1 & Don\#1 & 31.4 & & & & 420 & 143 & 2 & & 3.2 & 1 & & & & & & 1.55 & & & & & 32 \\
\hline BE8 & 212 & well & 31 & 9.1 & & 495 & 332 & & & & 1.1 & 0.6 & & & 150 & 69 & 8.7 & 1 & & & 0.02 & 6.4 & 42 \\
\hline BE9 & JIREC-3 & West Mesa \#2 & 30.6 & & & & 330 & 102 & 2 & & 4 & 1 & & & & & & 1.1 & & & & & 36 \\
\hline BE11 & 223 & well & 30 & 8.7 & & 660 & & & & & & & & & & & & 1.8 & & 0.38 & 0.06 & 3.23 & \\
\hline BE11 & 224 & woll & 30 & 8.6 & & 657 & & & & & & & & & & & & 2.2 & & 0.36 & 0.02 & 2.97 & \\
\hline $\mathrm{BE} 12$ & 225 & well & 30 & 8.8 & & 456 & & & & & & & & & & & & 1.2 & & 0.26 & 0.01 & 11.1 & \\
\hline BE13 & 227 & College\#2 & 30 & 8.5 & & 440 & & 100 & 1.4 & & 2.5 & 0.2 & & & & 62 & 6.9 & 1 & & & 0.03 & 10.6 & 30 \\
\hline CA2 & 120 & hot spring & 60.6 & 7.8 & & 767 & 505 & & & & 16 & 0.5 & & & 128 & 79 & 107 & 9.5 & & & & 0.4 & 81 \\
\hline $\mathrm{CA3}$ & GS22690 & Lower Frisco Hot Spring & & & & 1930 & & & & & & & & & 130 & 45 & 512 & 1.6 & & & & & 85 \\
\hline $\mathrm{CA} 3$ & GS31052 & Lower Frisco Hot Spring & 35.22 & 7.3 & & 1200 & & 200 & 0.2 & & & & 0.31 & & 135 & & 310 & & 0.4 & 0.2 & & & 73 \\
\hline CA3 & GS38864 & Lower Frisco Hot Spring & & & 7.6 & 1660 & & 280 & 16 & & & & & & 132 & 41 & 134 & 1.8 & & 0.1 & & & 76 \\
\hline CA3 & GS42605 & Lower Frisco Hot Spring & 46.11 & & \begin{tabular}{|l|l}
7.8 \\
\end{tabular} & 1780 & 1020 & 289 & & & 49 & 40 & & & 127 & & 460 & & & & & & \\
\hline CA3 & LFHS66-1 & Lower Frisco Hot Spring & 37.39 & & & & & 193 & 17 & & 55 & 9 & 0.24 & & & & & & & & & & \\
\hline CA3 & LFHS66-11 & Lower Frisco Hot Spring & 45.56 & & & & & 350 & 23 & & 103 & 8 & 0.59 & & & & & & & & & & \\
\hline CA3 & LFHS66-3B & Lower Frisco Hot Spring & 39.56 & & & & & 215 & 16 & & 50 & 7 & \begin{tabular}{|l|}
0.39 \\
\end{tabular} & & & & & & & & & & \\
\hline CA3 & LFHS66-4 & Lower Frisco Hot Spring & 41.22 & & & & & 275 & 19 & & 58 & 12 & 0.46 & & & & & & & & & & \\
\hline CA3 & LFHS66-7 & Lower Frisco Hot Spring & 45.11 & & & & & 265 & 23 & & 100 & 10 & 0.5 & & & & & & & & & & $\dot{-}$ \\
\hline CA3 & LFHS66-8 & Lower Frisco Hot Spring & 46.94 & & & & & 300 & 19 & & 65 & 6.4 & \begin{tabular}{|l|}
0.54 \\
\end{tabular} & & & & & & & & & & \\
\hline CA3 & LFHS66-9 & Lower Frisco Hot Spring & 49.44 & & 8.01 & 1940 & & 307 & 22 & & 65 & 5.7 & 0.59 & & 88 & & & 1.8 & & 0.078 & & & 93 \\
\hline CA3 & NB82-34 & Lower Frisco Hot Spring & 49 & 7.6 & & & & 296 & 17 & & 51 & & 0.46 & & & & & & & & & & 80 \\
\hline CA5 & 121 & Lower Frisco Hot Spring & 43 & 7.6 & & 1660 & & 280 & 16 & & & & & & 132 & 41 & 434 & 1.8 & & 0.001 & & 1.3 & 76 \\
\hline CA7 & 129 & Upper Frisco Hot Spring & 36.5 & 9.7 & & 284 & & 66 & 0.5 & & & & & & 57 & 6.6 & 5 & 1.0 & & 0.04 & & 0.6 & 58 \\
\hline CA7 & GS38851 & Upper Frisco Hot Spring & 36.67 & & 9.7 & 284 & & 66 & 0.5 & & & & & & 57 & 6.6 & 5 & 1 & & 0.04 & & & 58 \\
\hline CA7 & NB82-35 & Upper Frisco Hot Spring & 39 & 8.1 & & & & 71 & 0.24 & & 0.8 & & 0.01 & & & & & & & & & & 49 \\
\hline CA7 & UFHS2-66 & Upper Frisco Hot Spring & 36.67 & & 9.15 & 242 & 223 & 69 & 0.15 & & 3 & 0.1 & 0.1 & & 97 & & 12 & 2 & \begin{tabular}{|l|l|}
0.76 \\
\end{tabular} & 0.037 & & & 40 \\
\hline CA9 & 122 & Lower Frisco Hot Spring & 35 & 7.3 & & 1200 & & 200 & 12 & & & & 0.31 & & 135 & & 310 & & 0.4 & 0.2 & & & 73 \\
\hline CA10 & 168 & Pueblo Windmill & 33.8 & & & & 950 & 170 & 13 & & 130 & 29 & & & & 310 & 68 & 3.4 & & 0.36 & 1.6 & & 14 \\
\hline CA10 & 169 & Pueblo Windmill & 34 & 6.7 & 7.2 & 1600 & & 150 & 13 & & 150 & 31 & & & & 280 & 68 & & & 0.28 & 1.3 & & 15 \\
\hline CA10 & MYERS1 & Pueblo Windmil! & 34 & & 7.2 & 1600 & & 150 & 13 & & 150 & 31 & & & & 280 & 68 & & & & & & \\
\hline CA11 & FCS2-66 & Frieborn Canyon Hol Spring & 33.33 & & 8.44 & 150 & 151 & 39 & 0.2 & & 7 & 0.17 & & & 37 & & 7.4 & 1.3 & & 0.072 & & & 31 \\
\hline CA12 & NNS-BOIL & hot spring & 32.8 & & & & & 39 & 1 & & 15 & \begin{tabular}{|l|}
0.93 \\
\end{tabular} & 0.1 & & & & & & & & & & \\
\hline CA12 & NNS-CAS & hot spring & 34.4 & & & & & 39 & 1 & & 16.4 & 0.84 & 0.1 & & & & & & & & & & \\
\hline CA17 & MYERS2 & well & 28 & & 7.3 & 1440 & & 88 & 9.7 & & 180 & 41 & & & & 280 & 63 & 1 & & & & & \\
\hline CA17 & MYERS3 & well & 29 & & 7 & 1300 & & 86 & 9.3 & & 180 & 40 & & & & 290 & 63 & 0.9 & & & & & \\
\hline CA19 & MGSEEP & warm seep & 27.2 & & & & & 43 & 1.1 & & 14.1 & 0.73 & 0.05 & & & & & & & & & & \\
\hline CA21 & GS36104 & Gilla Middle Fork Pool HS & 52.2 & & 8.1 & 422 & & & & & & & & & 108 & 22 & 59 & & & & & & \\
\hline CA21 & NB82-33 & Gilla Middle Fork Pool HS & 65 & 7.6 & & & & 164 & 3.9 & & 17 & & 0.45 & & & & & & & & & & 73 \\
\hline CB3 & 180 & Acoma \#1 Well & 41.5 & 6.9 & 7.3 & 1300 & & 88 & 7.4 & & 140 & 39 & & & & 290 & 72 & 0.7 & & 0.28 & 0.72 & & 18 \\
\hline $\mathrm{CB3}$ & 181 & Acoma \#1 Well & 41.7 & 7 & 7 & 1350 & & 91 & 7.1 & & 140 & 40 & & & & 290 & 73 & 0.7 & & 0.27 & 0.32 & & 18 \\
\hline $\mathrm{CB} 3$ & 183 & Acoma \#1 Well & 40.5 & & 7.3 & 1270 & & 91 & 7.3 & & 140 & 40 & & & & 300 & 70 & 0.6 & & 0.25 & 0.49 & & 18 \\
\hline
\end{tabular}




\begin{tabular}{|c|c|c|c|c|c|c|c|c|c|c|c|c|c|c|c|c|c|c|c|c|c|c|c|}
\hline SITEID & SAMPLE & NAME & \begin{tabular}{|c|}
$T M P$ \\
$C$ \\
\end{tabular} & \begin{tabular}{|c|}
$\mathrm{pH}$ \\
field
\end{tabular} & \begin{tabular}{|l|}
$p H$ \\
$l a b$ \\
\end{tabular} & \begin{tabular}{|l|} 
COND \\
us $/ \mathrm{cm}$ \\
\end{tabular} & $\begin{array}{c}\text { TDS } \\
\text { mg/L } \\
\end{array}$ & $\begin{array}{c}\mathrm{Na} \\
\mathrm{mg} / \mathrm{L}\end{array}$ & $\begin{array}{c}K \\
m g h\end{array}$ & $\begin{array}{l}\mathrm{Na}+\mathrm{K} \\
\mathrm{mg} / \mathrm{L}\end{array}$ & $\begin{array}{c}\mathrm{Ca} \\
m g h \\
\end{array}$ & $\begin{array}{c}M g \\
m g / 2\end{array}$ & $\begin{array}{c}L \\
m g / 2\end{array}$ & $\begin{array}{c}S r \\
m g h\end{array}$ & $\begin{array}{l}\mathrm{HCO3} \\
\mathrm{mg} / \mathrm{L}\end{array}$ & $\begin{array}{l}\text { so4 } \\
\mathrm{mg} / \mathrm{L}\end{array}$ & $\begin{array}{c}\mathrm{Cl} \\
\mathrm{mgh}\end{array}$ & \begin{tabular}{c|}
$F$ \\
$m g h$
\end{tabular} & \begin{tabular}{|c|}
$\mathrm{Br}$ \\
$\mathrm{mg} / \mathrm{L}$
\end{tabular} & \begin{tabular}{|c|}
$B$ \\
$m g /$ \\
\end{tabular} & \begin{tabular}{c|}
$\mathrm{Fe}$ \\
$\mathrm{mgh}$ \\
\end{tabular} & \begin{tabular}{|l|} 
NO3 \\
mgl
\end{tabular} & \begin{tabular}{|l|}
$\mathrm{SIO2}$ \\
$\mathrm{mgh}$
\end{tabular} \\
\hline CB4 & 170 & well & 41 & 6.18 & 7.06 & 2800 & 2320 & 220 & 16 & & 350 & 77 & 0.73 & 4.7 & & 940 & 210 & 1.2 & 0.55 & 0.61 & 2.3 & & 21 \\
\hline $\mathrm{CB} 5$ & 177 & well & 38.1 & 6.45 & 6.87 & 3300 & 3180 & 110 & 13 & & 670 & 110 & 0.34 & 6.4 & & 2000 & 110 & 1.6 & 0.27 & 0.43 & 3.8 & & 16 \\
\hline CB7 & 184 & well & 34.5 & 6.42 & 7.44 & 5500 & 5360 & 810 & 35 & & 540 & 130 & 1.1 & 7.8 & & 3200 & 250 & 2.9 & 0.34 & 1.1 & 5.5 & & 15 \\
\hline CB8 & 171 & well & 34 & 6.44 & 6.77 & 8000 & 6280 & 1600 & 75 & & 580 & 110 & 6.1 & 6.3 & & 1400 & 1600 & 0.8 & 4.2 & 5.5 & 0.04 & & 16 \\
\hline CB10 & 179 & well & 33.1 & 6.65 & 7.22 & 4450 & 3910 & 610 & 48 & & 450 & 100 & 0.74 & 8.6 & & 2400 & 180 & 3.4 & 0.34 & 1.2 & 10 & & 15 \\
\hline CB12 & 226 & well & 32 & 8 & & 550 & 340 & 12 & 2 & & 74 & 25 & & & 250 & 84 & 7.5 & 0.4 & & & & 0.62 & \\
\hline CV1 & 95 & well & 33 & 7.1 & 7.9 & 900 & 651 & 16 & 1.2 & & 120 & 45 & & & & 270 & 12 & 1.5 & & 0.03 & 0.68 & 0.53 & 12 \\
\hline DA1 & GROSS3 & Chaffee 55-25 & 68 & & & & & 350.8 & 53.2 & & 96.9 & 33 & & & & & & & & & & & 51.5 \\
\hline DA1 & GROSS 4 & Chaffee 55-25 & 68 & & & & & 383.5 & 52 & & 156.7 & 33.5 & & & & & & & & & & & 54.5 \\
\hline DA3 & CLARYST & Clary and Ruther State 1 & 69.4 & & & & & & & & & & & & & & & & & 0.15 & & & \\
\hline DA4 & GROSS1 & Chaffee 35-25 & 68 & & & & & 391.5 & 54.7 & & 129.2 & 31.2 & & & & & & & & & & & 56.5 \\
\hline DA5 & $280 L C 2$ & LC-2 & 52.5 & & 7.41 & 2530 & 2004 & 376.3 & 5.5 & & 161.9 & 0.7 & & & 543 & 294.9 & 346.7 & & & & 7.13 & 1.28 & \\
\hline DA5 & GROSSB & LC-2 & 68 & & & & & 214.5 & 6.2 & & 10.2 & 1 & & & & & & & & & & & 24.3 \\
\hline DA6 & EXXONOLE & Exxon Beard Ole Federal & 65.5 & & & & & & & & & & & & & & & & & & & & \\
\hline DA7 & GROSS2 & Chaffee 12-24 & 65 & & & & & 392.4 & 58.3 & & 107.4 & 28 & & & & & & & & & & & 50.9 \\
\hline DA8 & $12581 \mathrm{PG} 3$ & NMSU PG-3 & \begin{tabular}{|l|}
63.3 \\
\end{tabular} & & & & 1981 & 488 & 52 & & 138 & \begin{tabular}{|l|}
17.4 \\
\end{tabular} & & & & & 546 & & & & 0.2 & & \\
\hline DAB & 1980PG3 & NMSUPG-3 & & & 6.25 & 3130 & & 488 & 52 & & 141 & 18.8 & & & 610 & 240 & 546 & & & & 5 & 0.02 & 73 \\
\hline DAB & GROSS6 & NMSUPG-3 & 63 & & & & & 488 & 52 & & 141 & 18.8 & & & & & & & & & & & 73 \\
\hline DAB & NDPG3 & NMSU PG-3 & & & & & 1748 & & & & & & & & 596.7 & 343 & & 2.28 & & & 0.15 & & \\
\hline DA9 & BTHSE & Radium Hot Springs Bailey's bathhouse & & & \begin{tabular}{|l|}
7.7 \\
\end{tabular} & & 874 & 1090 & & & 136 & 68.5 & & & & 259 & 1890 & 2.6 & & & & & \\
\hline DA9 & NMPHL316 & Radium Hot Springs Bailey's bathhouse & & & 7.2 & 6100 & & 1100 & 163 & & 131 & 15 & & & 341 & 269 & 1677 & 5.3 & & & & & \\
\hline DA10 & BINNS1 & Certified Sand Well & \begin{tabular}{|l|l|}
58.8 & \\
\end{tabular} & & & & & & & & & & & & & & & & & & & & \\
\hline DA12 & 69 & Radium Hol Springs Hotel Well \#2 & 85.5 & 6.9 & & 6060 & & & & 1200 & 140 & 23 & & & 430 & 260 & 1700 & 4.6 & & & & 2 & 71 \\
\hline DA12 & 70 & Radium Hol Springs Hotel Well \#2 & 52.5 & 6.7 & & 6100 & & 1100 & 170 & & & & 1.1 & & 444 & & 1600 & & 2.8 & 0.78 & & & 74 \\
\hline DA12 & 71 & Radium Hol Springs Hotel Well \#2 & 53 & 8.2 & & 6210 & & & & & & & & & 420 & 263 & 1630 & & & & & & \\
\hline DA12 & GS10160 & Radium Hol Springs Hotel Well \#2 & 85.56 & & 6.9 & 6060 & & & & & 142 & 23 & & & 427 & 265 & 1660 & 4.6 & & & & & 71 \\
\hline DA12 & GS31053 & Radium Hol Springs Hotel Well \#2 & & 6.7 & & 6100 & & 1100 & 170 & & & & 1.1 & & 444 & & 1600 & & 2.8 & 0.78 & & & 74 \\
\hline DA12 & GS35979 & Radium Hol Springs Hotel Woll \#2 & & & 8.2 & 6210 & & & & & & & & & 420 & 263 & 1630 & & & & & & \\
\hline DA12 & GS38867 & Radium Hot Springs Hotel Well \#2 & & & \begin{tabular}{|l|}
7.2 \\
\end{tabular} & 5540 & & 1100 & 155 & & & & & & 424 & 277 & 1660 & 5.2 & & 0.32 & & & 66 \\
\hline DA12 & NB82-21 & Radium Hol Springs Hotel Well \#2 & 53 & 6.8 & & & & 1120 & 173 & & & & 1.2 & & & & & & & & & & 64 \\
\hline DA12 & S76RSNMS & Radium Hol Springs Hotel Well \#2 & & & & & 3460 & & & & 130 & 13 & 4 & & 436 & 806 & 1610 & 5.3 & & & & & 70 \\
\hline DA15 & 4681PG2 & NMSUPG-2 & \begin{tabular}{|l|}
47.7 \\
\end{tabular} & & & & 2070 & 450 & 51 & & 188 & 21 & & & 508.9 & 226.2 & 610 & & & & & & \\
\hline DA15 & NB82-23 & NMSUPG-2 & 59 & 7.1 & & & & 450 & 55 & & 105 & & 0.48 & & & & & & & & & & 71 \\
\hline DA16 & 11 & Pure Oil Federal "H" 1 & 45 & 7.3 & & 7380 & & 1400 & & & & & & & 930 & 860 & 1600 & & & & & & \\
\hline DA16 & GS48858 & Pure Oil Federal "H" 1 & 45 & & & 7380 & & & & & & & & & & & & & & & & & \\
\hline DA20 & 17 & well & 42.5 & 8.6 & 7.9 & 10900 & 6990 & 2100 & 19 & & 290 & 10 & & & & 1700 & 2700 & 2.6 & & & & & 63 \\
\hline DA20 & 18 & well & 35 & \begin{tabular}{|l|}
8.7 \\
\end{tabular} & 8.8 & 2100 & 1350 & 370 & 13 & & 20 & 12 & & & & 180 & 400 & 0.9 & & & \begin{tabular}{|l|}
0.29 \\
\end{tabular} & & 52 \\
\hline DA20 & 19 & well & \begin{tabular}{|l|}
31.5 \\
\end{tabular} & \begin{tabular}{|l|}
8.6 \\
\end{tabular} & 8.7 & 1580 & 955 & 280 & 6.6 & & 25 & 16 & & & & 160 & 200 & 0.9 & & & & & 67 \\
\hline DA22 & 46 & Tellyer Woll & 36.5 & 7.5 & & 1020 & & 180 & & & 66 & 13 & & & 319 & 140 & 170 & & & & & & \\
\hline DA22 & TELLYER1 & Tellyer Well & \begin{tabular}{|l|}
36.5 \\
\end{tabular} & & 7.5 & 1020 & & 180 & & & 66 & 13 & & & 319 & & 170 & & & & 2 & & \\
\hline DA25 & S76NMS112 & well & 35.56 & & 7.5 & 1800 & 1020 & 375 & 15 & & & & & & 222 & 168 & 401 & & & & & & \\
\hline DA26 & $2 \mathrm{GD1}$ & NMSU GD-1 & & & & & 1550 & 280 & 12 & & 185 & 16 & & & & 185 & 432 & & & & & & 12 \\
\hline DA26 & 3GD1 & NMSU GD-1 & & & & & 1200 & 288 & 39 & & & & & & & 292 & 375 & & & & & & \\
\hline DA26 & 4GD1 & NMSU GD-1 & & & & & & 184 & 22 & & 57 & \begin{tabular}{|l|}
7.2 \\
\end{tabular} & & & & & 137 & & & & 0.26 & & \\
\hline DA26 & $5 \mathrm{GD1}$ & NMSU GD-1 & & & & & & & & & 60 & 7.5 & & & & & & & & & 9.8 & & \\
\hline DA26 & NB82XX & NMSU GD-1 & 30 & & & & & 240 & 26 & & 680 & & \begin{tabular}{|l|}
0.26 \\
\end{tabular} & & & & & & & & & & 50 \\
\hline DA27 & S76LASWH & White well & \begin{tabular}{|l|}
34.5 \\
\end{tabular} & & 8.15 & 1460 & 1000 & 275 & 32 & & 17 & 12.3 & 0.25 & & 242 & & 580 & 2.7 & 1.11 & 0.35 & & & 49 \\
\hline DA33 & GS28683 & well & \begin{tabular}{|l|}
32.22 \\
\end{tabular} & & 8.6 & 4640 & 2930 & & & & 110 & \begin{tabular}{|l|}
1.1 \\
\end{tabular} & & & 27 & 927 & 910 & & & & & & 19 \\
\hline DA34 & 43 & well & 32 & 8.7 & & 2320 & & & & & 24 & 1.9 & & & 33 & 590 & 320 & 5.9 & & & 0.03 & 1.3 & 31 \\
\hline
\end{tabular}




\begin{tabular}{|c|c|c|c|c|c|c|c|c|c|c|c|c|c|c|c|c|c|c|c|c|c|c|c|}
\hline SITEID & SAMPLE & NAME & \begin{tabular}{|c|}
$T M P$ \\
$C$ \\
\end{tabular} & $\begin{array}{c}\mathrm{pH} \\
\text { fleld }\end{array}$ & $\begin{array}{l}p H \\
l a b\end{array}$ & $\begin{array}{l}\text { COND } \\
\text { uS } / \mathrm{cm}\end{array}$ & $\begin{array}{c}\text { TDS } \\
\text { mg/h }\end{array}$ & $\begin{array}{c}\mathrm{Na} \\
\mathrm{mg} / \mathrm{L}\end{array}$ & $\begin{array}{c}K \\
m g /\end{array}$ & $\begin{array}{l}\mathrm{Na}+K \\
\mathrm{mg} / \mathrm{L}\end{array}$ & $\begin{array}{c}\mathrm{Ca} \\
\mathrm{mg} / \mathrm{L}\end{array}$ & \begin{tabular}{|c|}
$\mathrm{Mg}$ \\
$\mathrm{mg} / \mathrm{h}$
\end{tabular} & $\begin{array}{c} \\
m g / \\
\end{array}$ & $\begin{array}{c}s r \\
m g / L\end{array}$ & $\begin{array}{l}\mathrm{HCO3} \\
\mathrm{mg} / \mathrm{L}\end{array}$ & $\begin{array}{l}\text { sod } \\
m g / 2\end{array}$ & $\begin{array}{c}\mathrm{Cl} \\
\mathrm{mg} / \mathrm{L}\end{array}$ & \begin{tabular}{c|}
$F$ \\
$m g / 2$
\end{tabular} & $\begin{array}{c}B r \\
m g h\end{array}$ & \begin{tabular}{|c|}
$B$ \\
$m g h$ \\
\end{tabular} & \begin{tabular}{|c|}
$F e$ \\
$m g / L$
\end{tabular} & \begin{tabular}{l|}
$\mathrm{NO3}$ \\
$\mathrm{mgh}$
\end{tabular} & \begin{tabular}{|l|}
$\mathrm{S1O2}$ \\
$\mathrm{mg} / \mathrm{L}$
\end{tabular} \\
\hline DA36 & S76BERING & Berino Well & & & 7.9 & 1080 & 740 & 202.4 & 0.58 & & 30 & & & & 367.5 & 155 & 68.1 & 1.25 & & 0.31 & & & \\
\hline DA40 & 21 & well & 31 & 7.8 & & 804 & & & & & 20 & 0.5 & & & 160 & 140 & 79 & & & & & & \\
\hline DA41 & 2 & well & 31 & 7.8 & 7.8 & 5700 & 3780 & 1200 & 9.1 & & 84 & 24 & & & & 1000 & 1200 & 1 & & & 0.03 & & 34 \\
\hline DA44 & 48 & $\mathrm{SC2}$ & 30.5 & & & 270 & & & & & & & & & & & & & & & & & \\
\hline DA45 & 12 & well & 30.5 & 8 & 7.4 & 2200 & 1300 & 390 & 3.4 & & 76 & 0.6 & & & & 300 & 460 & 0.8 & & & 0.03 & & 45 \\
\hline DA45 & 14 & weil & 30.5 & 8.9 & 8.6 & 1000 & 656 & 210 & 2.8 & & 9.7 & 1.1 & & & & 190 & 44 & 2.2 & & & 0.021 & & 42 \\
\hline DA46 & 52 & $T-18$ & 30 & 7.4 & 7.9 & 686 & & 110 & 3.2 & & 32 & 3.2 & & & & 150 & 43 & 4.5 & & 0.1 & 0.036 & 0.49 & 26 \\
\hline DA46 & 53 & $T-18$ & 30 & & 7.9 & 710 & & & & & & & & & & & & & & & & & \\
\hline DA50 & HUNTWATW & Radium Springs College Ranch Windmill & & & 7.69 & & & 250.4 & 6.6 & & 75.4 & 2.7 & & & 97.6 & 616.9 & & & & & & & \\
\hline DA52 & $21 \mathrm{H} 0600$ & Radium Springs Masson 21 & & & 8 & & & 1100 & 166 & & 129 & 13 & 1.19 & & 444 & 283 & 1550 & 5.6 & & 0.82 & 0.32 & & \\
\hline DA52 & $21 \mathrm{H0900}$ & Radium Springs Masson 21 & & & 8.1 & & & 1025 & 164 & & 128 & 13 & 1.19 & & 441 & 265 & 1600 & 5.5 & & 0.77 & 0.14 & & \\
\hline DA52 & $21 \mathrm{H} 1800$ & Radium Springs Masson 21 & & & 8 & & & 1050 & 162 & & 128 & 13 & 1.18 & & 444 & 248 & 1610 & 5.7 & & 0.78 & 0.34 & & \\
\hline DA52 & 21TRUN & Radium Springs Masson 21 & & & 7.9 & & & 1075 & 161 & & 126 & 13 & 1.2 & & 454 & 262 & 1710 & 6.2 & & 0.8 & 0.27 & & \\
\hline DA53 & 89MASS22 & Radium Springs Masson 22 & & & & & & & & & & & & & & & 1683 & & & & & & \\
\hline DA53 & SWL1295 & Radium Springs Masson 22 & & & & & & & & & & & & & & & 1720 & & & & & & \\
\hline DA54 & 87MASS23 & Radium Springs Masson 23 & & & & & & & & & & & & & & & 1764.4 & & & & & & \\
\hline DA54 & 89MASS23 & Radium Springs Masson 23 & & & & & & & & & & & & & & & 1669 & & & & & & \\
\hline DA54 & SWL1296 & Radium Springs Masson 23 & & & & & & & & & & & & & & & 1764 & & & & & & \\
\hline DA54 & SWL5478 & Radium Springs Masson 23 & & & & & & 1250.2 & 144.7 & & 128.4 & 12.9 & & & 454 & 266.9 & 1823 & & & & & & \\
\hline DA55 & 311801 & Radium Springs Masson 26 & & & 6.66 & & & 863 & 163 & & 94.6 & 15.4 & & & & & 1833 & 4.6 & & 0.83 & 0.036 & & \\
\hline DA56 & 1RYAN7235 & Radium Springs Ryan 72-35 & & & & & 2584 & 690 & 115 & & 117 & 14 & 0.68 & & 286 & 239 & & 4.64 & & 0.61 & & & 50.3 \\
\hline DA57 & GROSS7 & NMSU GD-2 & 42 & & & & & 386.2 & 34.8 & & 114.5 & 36.6 & & & & & & & & & & & 36 \\
\hline DA58 & NMSUOW1 & NMSU OW-1 & & & & & 1765 & 424 & & & 110 & & & & & & 466 & 1.48 & & & 0.81 & & \\
\hline DA59 & GROSS5 & NMSU PG-1 & 61 & & & & & 480.2 & 57.9 & & \begin{tabular}{|l|}
155.7 \\
\end{tabular} & 29.7 & & & & & & & & & & & 92 \\
\hline DA59 & N10PG1 & NMSU PG-1 & & & 6.45 & 3720 & 2044 & 500.7 & 54.1 & & \begin{tabular}{|l|}
232.7 \\
\end{tabular} & 43.2 & & & 458.8 & 308 & 847.1 & 2.54 & & & 7.71 & & \\
\hline DA59 & N4PG1 & NMSU PG-1 & & & 6.17 & 6590 & 4220 & 627.2 & 80.5 & & \begin{tabular}{|l|}
538.4 \\
\end{tabular} & 108 & & & 134.2 & 645 & 1714.8 & 3.51 & & & 81.6 & 0.04 & \\
\hline DA59 & N7PG1 & NMSU PG-1 & & & 6.6 & 4510 & 2868 & 536.3 & 61.1 & & 325.9 & 60.4 & & & \begin{tabular}{|l|}
341.7 \\
\end{tabular} & 402.5 & 1123.2 & 2.7 & & & 13.03 & & \\
\hline DA59 & NBPG1 & NMSU PG-1 & & & 6.64 & 4050 & 2416 & 504.6 & 55.2 & & 267.6 & 49.4 & & & 433.2 & 292.5 & 963 & 2.41 & & & \begin{tabular}{|l|}
9.31 \\
\end{tabular} & & \\
\hline DA59 & NB82-22 & NMSU PG-1 & 61 & 6.8 & & & & 386 & 54 & & 82 & & 0.51 & & & & & & & & & & 73 \\
\hline DA59 & PG1PART1 & NMSUPG-1 & & & & & 1936 & 484 & 54 & & 116 & 19 & & & & & & & & & & & 70 \\
\hline DA59 & PG1PART2 & NMSU PG-1 & & & & & 1904 & 488 & & & & 19 & & & & & & & & & & & 68 \\
\hline DA59 & PG1PART3 & NMSU PG-1 & & & 6.3 & 3110 & & 488 & 54 & & 143 & 18.6 & & & 620 & 250 & 584 & 1.3 & & & 2.8 & 0.03 & \\
\hline DA60 & BAIL370 & NMSUPG-4 & & & 8.47 & 2450 & 1636 & 389 & 35 & & 107 & 25.1 & & & 593 & 250 & 341 & 1.57 & & & 0.83 & 0.54 & \\
\hline DA60 & DRILL13 & NMSU PG-4 & & 6.55 & 6.72 & 2800 & 1818 & 428 & 74 & & 132 & 32.1 & & & 487 & 251 & 570 & 1.78 & & & 0.22 & & \\
\hline DA60 & DST1PG4 & NMSU PG-4 & 63 & & 7.7 & 2720 & 1695 & 430.9 & 59 & & \begin{tabular}{|l|}
102.9 \\
\end{tabular} & 31.4 & & & 462.5 & 232.4 & 528.3 & 2 & & & 0.05 & 0.05 & \\
\hline DA60 & DST4PG4 & NMSU PG-4 & 63 & & 7.56 & 2790 & 1854 & 449.1 & 48.8 & & \begin{tabular}{|l|}
107.4 \\
\end{tabular} & 32.6 & & & 489.4 & 289.2 & 528.3 & 2.08 & & & & 0.03 & \\
\hline DA60 & WWEINC1 & NMSU PG-4 & & & 6.6 & 2520 & & 280.7 & & & 152 & 57.1 & & & 442 & 100 & 533 & & & & 0.13 & & 22.9 \\
\hline DA60 & PTOPG4 & NMSU PG-4 & & & 6.64 & & 1770 & 428 & 49.2 & & \begin{tabular}{|l|}
158.7 \\
\end{tabular} & 31.2 & & & 603 & 248 & 541 & 2.16 & & & 1.17 & & \\
\hline ED1 & 81 & Clayton Well & 31 & & & 3030 & & & & & & & & & & & 210 & & & & & & \\
\hline ED1 & 82 & Clayton Well & 31 & & & 3030 & & & & & & & & & & & 210 & & & & & & \\
\hline GR1 & GS15106 & Mimbres Hot Springs \#3 & 61.11 & 8.3 & & 450 & & 94 & 1.3 & & 3.3 & 0.6 & 0.1 & & 107 & 65 & 17 & 18 & & 0.05 & & & 56 \\
\hline GR1 & MIML3-12-65 & Mimbres Hot Springs \#3 & 62.28 & & 8.56 & 430 & 221 & 138 & 0.96 & & 3 & & 0.12 & & 85 & & 22 & 3.17 & 0.92 & 2.04 & & & 60 \\
\hline GR1 & MIML3-8-65 & Mimbres Hot Springs \#3 & & & 8.73 & 422 & 285 & 95 & 0.9 & & 2.25 & & 0.09 & & 89 & & 20 & 3.05 & 0.12 & & & & 51 \\
\hline GR5 & GHS0920 & Gila Spring Hot Spring \#14 & 66 & 7.75 & & 640 & & & & & & & & & 109 & & & & & & & & 65 \\
\hline GR6 & NB82-32 & Gila HS northern artesian well & 65 & 7.6 & & & & 148 & 3.7 & & 10 & & 0.24 & & & & & & & & & & 71 \\
\hline GR7 & 115 & Gila Hot Springs & 64 & 8.2 & & 653 & 369 & & & & 11 & 0.2 & & & 110 & 40 & 100 & 12 & & 0.07 & 0.04 & 0.5 & 33 \\
\hline GR7 & 117 & Gila Hol Springs & 61 & 7.5 & & 620 & & 120 & 3.5 & & & & 0.22 & & 101 & & 100 & & 0.2 & 0.1 & & & 72 \\
\hline GR7 & GHS-66 & Gila Hot Springs & 41.5 & & 8.10 & 560 & 496 & 127 & 3.6 & & 11 & & 0.27 & & 96 & & 104 & 3.4 & 0.18 & 0.19 & & & 74 \\
\hline GR7 & GHS-74 & Gila Hot Springs & 63.89 & 7.5 & & 620 & & 120 & 3.5 & & & & 0.22 & & 101 & & & & 0.2 & 0.1 & & & 72 \\
\hline
\end{tabular}




\begin{tabular}{|c|c|c|c|c|c|c|c|c|c|c|c|c|c|c|c|c|c|c|c|c|c|c|c|}
\hline SITEID & SAMPLE & NAME & $\begin{array}{c}T M P \\
C\end{array}$ & \begin{tabular}{c|}
$p H$ \\
ffeld
\end{tabular} & \begin{tabular}{|l|}
$p H$ \\
$a b$ \\
\end{tabular} & $\begin{array}{l}\text { COND } \\
\mathrm{uS} / \mathrm{cm}\end{array}$ & $\begin{array}{l}\text { TDS } \\
\mathrm{mgh}\end{array}$ & $\begin{array}{c}\mathrm{Na} \\
\mathrm{mg} /\end{array}$ & $\begin{array}{c}K \\
m g / L\end{array}$ & $\begin{array}{l}\mathrm{Na}+K \\
\mathrm{mg} / \mathrm{L}\end{array}$ & $\begin{array}{c}\mathrm{Ca} \\
\mathrm{mg} / \mathrm{L}\end{array}$ & $\begin{array}{c}M g \\
m g \Omega\end{array}$ & $\begin{array}{c} \\
m g h\end{array}$ & $\begin{array}{c}S r \\
m g h\end{array}$ & $\begin{array}{l}\mathrm{HCO} 3 \\
\mathrm{mg} / \mathrm{L}\end{array}$ & $\begin{array}{l}\text { SO4 } \\
\text { mg/L }\end{array}$ & $\begin{array}{c}\mathrm{Cl} \\
\mathrm{mg} / \mathrm{L}\end{array}$ & $\begin{array}{c}F \\
m g / L\end{array}$ & \begin{tabular}{|c|}
$\mathrm{Br}$ \\
$\mathrm{mg} / \mathrm{h}$ \\
\end{tabular} & \begin{tabular}{|c|}
$B$ \\
$m g h$ \\
\end{tabular} & $\begin{array}{c}\mathrm{Fe} \\
\mathrm{mgh}\end{array}$ & \begin{tabular}{|l|} 
NO3 \\
mg/h
\end{tabular} & \begin{tabular}{|l|}
$\mathrm{SiO2}$ \\
$\mathrm{mg} / \mathrm{L}$
\end{tabular} \\
\hline GR7 & GS36105 & Gila Hot Springs & 63.9 & & 8.20 & 653 & 369 & & & & 11 & 0.2 & & & 109 & 40 & 104 & 12 & & 0.07 & & & 33 \\
\hline GR7 & NB82-31 & Gila Hot Springs & 59 & 7.4 & & & & 135 & 3.4 & & 13 & & 0.26 & & & & & & & & & & 68 \\
\hline GR9 & NB82-30 & Gila HS middle artesian well & 62 & 7.6 & & & & 142 & 3.5 & & 57 & & 0.26 & & & & & & & & & & 71 \\
\hline GR10 & GHS0711 & Gila Hot Springs Campbell $\$ 2$ Well & 61 & & & & & & & & & & & & & & & & & & & & \\
\hline GR10 & GHS14006 & Gila Hot Springs Campbell \#2 Well & 62 & 7.72 & & 550 & & & & & & & & & 99 & & & & & & & & 63 \\
\hline GR10 & GHS1445 & Gila Hot Springs Campbell \#2 Well & 61 & 7.97 & & 620 & & & & & & & & & 102 & & & & & & & & 66 \\
\hline GR10 & GHS1610 & Gila Hot Springs Campbell \#2 Woll & 62 & 7.97 & & 590 & & & & & & & & & 102 & & & & & & & & 68 \\
\hline GR10 & GHS2252 & Gila Hot Springs Campbell \#2 Well & 60 & & & & & & & & & & & & & & & & & & & & \\
\hline GR11 & MIM-L25 & Mimbres Hot Springs \#25 & 59 & & 8.31 & & & 113 & 1.2 & & 2.5 & & 0.12 & & 89 & & 19 & 3.1 & \begin{tabular}{|l|}
0.47 \\
\end{tabular} & 0.15 & & & 56 \\
\hline GR12 & MIM-L28 & Mimbres Hot Springs \#28 & 58.06 & & & 452 & 308 & & & & 12 & 2.6 & & & 77 & 65 & 17 & 16 & & & & & 53 \\
\hline GR12 & MIM-L32 & Mimbres Hot Springs \#28 & 45.33 & & 8.35 & & & 112 & 0.95 & & 3 & & 0.13 & & 65 & & 21 & 3.2 & 0.28 & & & & 45 \\
\hline GR13 & GHS0929 & Gila Spring Hot Spring \#41 & 58 & 7.85 & & 560 & & & & & & & & & 104 & & & & & & & & 56 \\
\hline GR14 & 85 & Mimbres Hot Springs & 58 & & & 450 & & & & & 12 & 2.6 & & & 75 & & 16 & 16 & & & & & 53 \\
\hline GR14 & NB82-25 & Mimbres Hot Springs & 58 & 7.9 & & & & 111 & 1.3 & & 2.4 & & 0.13 & & & & & & & & & & 55 \\
\hline GR15 & GS19645 & Mimbres Hot Springs \#8 & 57.5 & & & 450 & & & & & & & & & 75 & & 16 & 16 & & & & & \\
\hline GR17 & MiM-L21 & Mimbres Hot Springs \#21 & 55 & & 8.34 & & & 81 & 1.1 & & 1 & & & & 85 & & 20 & 3.3 & 0.31 & 1.11 & & & 46 \\
\hline GR18 & MIM-L23 & Mimbres Hot Springs \#23 & 54.44 & & & & & 127 & 2.7 & & 10 & 0.2 & 0.15 & & & & & & & & & & \\
\hline GR19 & 75 & Faywood Hot Springs & 52 & & & 560 & & & & & & & & & & & & & & & & & \\
\hline GR19 & FAY-S76-2 & Faywood Hot Springs & & & 8.7 & 560 & 384 & 94 & 8 & & 36.9 & 6.9 & 0.17 & & 214 & & 22 & 3.3 & 1.97 & 0.15 & & & 67 \\
\hline GR19 & GS19824 & Faywood Hot Springs & & & & 606 & & & & & & & & & 282 & 50 & 18 & 7 & & & & & \\
\hline GR19 & GS27917 & Faywood Hot Springs & 53.33 & & & 600 & & & & & 37 & 8.5 & & & 282 & & 17 & & & & & & \\
\hline GR19 & NB82-26 & Faywood Hot Springs & 55 & 8 & & & & 98 & 7.7 & & 47 & & 0.15 & & & & & & & & & & 40 \\
\hline GR20 & GS36104 & Gila Lyons Lodge swimming pool HS & & & 8.1 & 432 & & & & & & & & & 108 & 22 & 59 & & & & & & \\
\hline GR20 & SPS-LL66 & Gila Lyons Lodge swimming pool HS & 51.7 & & 8.35 & 390 & 335 & 105 & 2 & & 10 & 0.1 & 0.13 & & 101 & & 67 & 3.4 & \begin{tabular}{|l|}
0.67 \\
\end{tabular} & 1.72 & & & 58 \\
\hline GR21 & 114 & well & 52 & 8.1 & & 432 & & & & & & & & & 108 & 22 & 59 & & & & & & \\
\hline GR22 & WFALLHS & Gila River Waterfall HS & 44.72 & & 8.31 & 680 & 518 & 150 & 3.9 & & 18 & 0.35 & 0.25 & & 112 & & & 2.9 & 0.37 & 0.28 & & & 70 \\
\hline GR23 & EFGR-SUM & Gila East Fork no name spring & 41.39 & & 8.45 & 560 & 367 & 111 & 1.7 & & 11 & 0.2 & 0.23 & & 77 & & & 3.6 & 0.23 & 0.33 & & & 74 \\
\hline GR26 & EFS-LL66 & Gila Lyons Lodge East Fork Hot Springs & 35.56 & & 8.09 & 500 & 358 & 105 & 3.8 & & 14 & 0.65 & 0.2 & & 85 & & 91 & 3.2 & 0.11 & 0.083 & & & 49 \\
\hline GR26 & WSC-LL66 & Glla Lyons Lodge HS composile & & & 8.25 & 580 & 407 & 126 & 1.3 & & 10 & & 0.26 & & 83 & & & 3.5 & 0.28 & 3.4 & & & 67 \\
\hline GR26 & WSD-LL66 & Gila Lyons Lodge HS downstream & & & & & & 128 & 1.7 & & 9 & 0.1 & 0.21 & & & & & & & & & & \\
\hline GR26 & WSM-LL66 & Gilla Lyons Lodge HS midpoint & 45.56 & & & & & 119 & 1.7 & & 10.1 & 0.1 & 0.21 & & & & & & & & & & \\
\hline GR26 & WSU-LL.66 & Gila Lyons Lodge HS upstream & & & & & & 117 & 1.7 & & 20 & 0.1 & 0.21 & & & & & & & & & & \\
\hline GR27 & KWS-W3-1 & Kennecott Warm Spring Well \#3 & 34.55 & & 8.29 & 350 & 281 & 40 & 5.2 & & 37 & 13 & & & 205 & & 14 & 2.2 & 1.5 & 0.23 & & & 50 \\
\hline GR27 & KWS-W3-2 & Kennecott Warm Spring Well \#3 & & & 8.00 & 482 & 320 & 33.4 & 4.3 & 46 & 10.9 & & & 185.4 & & 10.9 & 2 & & & & & & \\
\hline GR29 & NB82-27 & Kennecott Warm Spring Well \#3 & 34 & 6.7 & & & & 51 & 4.6 & & 53 & & 0.07 & & & & & & & & & & 50 \\
\hline GR30 & 94 & Cliff Warm Well & 33.5 & 9 & & 665 & 435 & & & & 3 & 0.1 & & & 130 & 100 & 18 & 21 & & & & 0.1 & 48 \\
\hline GR30 & GS50019 & Cliff Warm Woll & 33.3 & & 9.00 & 665 & 435 & & & & 3 & 0.1 & & & 130 & 103 & 18 & 21 & & & & & 48.00 \\
\hline GR30 & S76CLIFF & Cliff Warm Well & 35.0 & & 8.50 & 610 & 439 & 153 & 1 & & 2 & & 0.2 & & 177 & & 20 & 3.4 & 0.53 & 0.75 & & & 44.4 \\
\hline GR34 & 87 & well & 32 & & & 754 & & & & & 86 & 19 & & & 240 & 190 & 11 & 1.6 & & & 0.03 & $\overline{0.8}$ & 18 \\
\hline GR35 & 84 & well & 31.1 & 7.1 & & 421 & 272 & 34 & & & 38 & 12 & & & 202 & 31 & 11 & 1.2 & & & & 3.4 & 35 \\
\hline GR36 & GS29750 & Spring Canyon Warm Spring & 28.89 & & 7.9 & 472 & 311 & & & & 18 & 3 & & & 232 & 38 & 8.5 & 6 & & & & & 32 \\
\hline GR39 & GS29790 & Riverside Well & 30 & & 7.9 & 551 & 363 & & & & 7.5 & 1 & & & 241 & 51 & 16 & 8 & & & & & 48 \\
\hline GR39 & NB82-28 & Riverside Well & 38 & 6.8 & & & & 138 & 1.8 & & 21 & & 0.14 & & & & & & & & & & 39 \\
\hline GR43 & T72P193 & Cliff Warm Spring & 25 & & 8.7 & 256 & & & & & & & & & 123 & & 5.2 & & & & & & \\
\hline GR44 & ATWS-W4-1 & Apache Tejo Warms Springs Well \#4 & & & 8.43 & 520 & 370 & 48 & 4.6 & & 34 & 17 & 0.1 & & 216 & & 19 & 2.7 & 0.72 & 0.84 & & & 36 \\
\hline GR44 & ATWS-W4-2 & Apache Tejo Warms Springs Well \#4 & & & 7.9 & 634 & 868 & 44.9 & 3.5 & & 72 & 10.4 & & & 268.9 & 70 & 16.3 & 1.9 & & 1 & & & \\
\hline GR45 & ATWS-W5 & Apache Tejo Warms Springs Well \#5 & & & & & & 47 & 4.6 & & 44 & 17 & & & & & & & & & & & \\
\hline HD2 & 35 & Lightning Dock hot well & 98 & & & 1540 & & & & & 19 & 1.2 & & & 170 & 460 & 78 & 11 & & 0.45 & & 0.9 & 140 \\
\hline $\mathrm{HO} 3$ & NB82-40 & Lightning Dock hot well & 96 & 6.8 & & & & 299 & 22 & & 29 & & 0.74 & & & & & & & & & & 106 \\
\hline
\end{tabular}




\begin{tabular}{|c|c|c|c|c|c|c|c|c|c|c|c|c|c|c|c|c|c|c|c|c|c|c|c|}
\hline SITEID & SAMPLE & NAME & \begin{tabular}{|c|}
$T M P$ \\
$C$ \\
\end{tabular} & $\begin{array}{c}\mathrm{pH} \\
\text { field }\end{array}$ & \begin{tabular}{|l|}
$\mathrm{pH}$ \\
$\mathrm{lab}$ \\
\end{tabular} & $\begin{array}{l}\text { COND } \\
\mathrm{uS} / \mathrm{Cm} \\
\end{array}$ & $\begin{array}{l}\text { TDS } \\
m g / L\end{array}$ & $\begin{array}{c}\mathrm{Na} \\
\mathrm{mg} / \mathrm{L}\end{array}$ & $\begin{array}{c}K \\
m g / L\end{array}$ & $\begin{array}{l}\mathrm{Na}+K \\
\mathrm{mg} / \mathrm{K}\end{array}$ & $\begin{array}{c}\mathrm{C}_{\mathrm{B}} \\
\mathrm{mg} / \mathrm{L}\end{array}$ & \begin{tabular}{c|}
$M g$ \\
$m g h$
\end{tabular} & $\begin{array}{c}4 \\
m q / 2\end{array}$ & $\begin{array}{c}S r \\
m g / L\end{array}$ & $\begin{array}{l}\mathrm{HCO} 3 \\
\mathrm{mg} / \mathrm{L}\end{array}$ & $\begin{array}{l}\mathrm{sa4} \\
\mathrm{mg} / \mathrm{L}\end{array}$ & $\begin{array}{c}\mathrm{Cl} \\
\mathrm{mg} / \mathrm{L}\end{array}$ & $\begin{array}{c} \\
m g / 2\end{array}$ & $\begin{array}{c}B r \\
m g / L\end{array}$ & \begin{tabular}{|c|}
$B$ \\
$m g / L$ \\
\end{tabular} & \begin{tabular}{c|c|}
$F e$ \\
$m g / L$
\end{tabular} & \begin{tabular}{|l|}
$\mathrm{NO3}$ \\
$\mathrm{mgh}$
\end{tabular} & $\begin{array}{l}\mathrm{SIO2} \\
\mathrm{mgh}\end{array}$ \\
\hline HD6 & 37 & Lightning Dock hot well & 94 & 7.6 & & 1510 & & & & & 22 & 1.5 & & & 160 & 460 & 80 & 13 & & & & 0.2 & 140 \\
\hline HD10 & S76LD-2 & Lightning Dock hot well & & & 8.25 & & 1055 & & & & & & & & & 480 & 100 & & & & & & \\
\hline HD10 & S76LD-3 & Lightning Dock hot well & & & 7.1 & 1577 & 1112 & 300 & 25 & & 28 & 11 & & & 198 & 463 & 102 & & & & & & \\
\hline HD10 & S76LD-4 & Lightning Dock hot well & & & 8.25 & 2200 & 1761 & 370 & 32 & & 70 & 4.9 & 0.9 & & & & 113 & & 0.37 & 0.37 & & & 106 \\
\hline HD11 & S76P15-1 & warm well & 35 & & & & & & & & & & & & & & & & & & & & \\
\hline HD11 & S76P15-2 & warm well & \begin{tabular}{|l|}
31.1 \\
\end{tabular} & & 7.8 & 1590 & & & & & & & & & 431 & & 102 & & & & & & \\
\hline HD11 & S76P15-3 & warm well & 31.39 & & & & & 91 & 2.1 & & 265 & 6.9 & & & & & & & & & & & \\
\hline HD13 & 1 & well & 32 & 7.4 & & 1020 & & & & & & & & & 340 & & 55 & & & & & & \\
\hline HD14 & 63 & Blowing Well & 31 & 7.8 & & 1590 & & & & & & & & & 430 & & 100 & & & & & & \\
\hline HD15 & 68 & well & 30 & 7.9 & 7.7 & 460 & & 40 & 2.4 & & 31 & 10 & & & & 12 & 17 & 0.5 & & 0.05 & 0.023 & 8.41 & 38 \\
\hline HD17 & S76LD-11 & Lightning Dock hot well & & & 7.6 & 1510 & 1110 & & & & 22 & 1.5 & & & 157 & 459 & 80 & 13 & & & & & 135 \\
\hline HD18 & S76LD-13 & Lightning Dock hot well & & & & & & 347 & 24 & & 20 & 0.1 & 0.8 & & & & & & & & & & \\
\hline HD19 & S76LD-14 & Lightning Dock hot well & & & 7.8 & 835 & 620 & 150 & 8 & & 28 & 11 & & & 228 & 169 & 42 & & & & & & \\
\hline HD20 & S76LD-7A & Lightning Dock hot well & & & & 1650 & 1020 & & & & 24 & 1.5 & & & 146 & 509 & 85 & & & & & & \\
\hline HD20 & S76LD-7B & Lightning Dock hot well & & & & 1540 & 1130 & & & & 19 & 1.2 & & & 181 & 460 & 78 & 11 & & 0.45 & & & 141 \\
\hline $\mathrm{HD} 20$ & S76LD-8 & Lightning Dock hot well & & & & 1600 & & & & & & & & & 163 & & 81 & & & & & & \\
\hline $\mathrm{HD2O}$ & S76LD-9 & Lightning Dock hot well & & & \begin{tabular}{|l|}
8.2 \\
\end{tabular} & 1600 & & & & & & & & & 163 & & 82 & & & & & & \\
\hline LU1 & 42 & SmyerWell & 39 & 9.6 & & 439 & & & & 100 & 4 & 0.6 & & & 120 & 34 & 9 & 1.7 & & & & 3.2 & 73 \\
\hline LU1 & S76-24SRTW & Smyer Well & 38.89 & & 9.6 & 439 & & & & & 4 & 0.6 & & & 0.23 & 34 & 9 & 1.7 & & & & & 73 \\
\hline LU3 & 41 & well & 34.5 & & & 515 & & & & 100 & 11 & 2 & & & 150 & 66 & 30 & 3.2 & & & & 3 & \\
\hline LU4 & 6 & well & 33 & & & 1620 & & & & & 5.2 & 3.6 & & & 420 & 230 & 130 & 9 & & & & 1.6 & 66 \\
\hline LU6 & 40 & well & 31.5 & & & 824 & & & & 180 & 6.5 & 3.5 & & & 210 & 77 & 53 & 18 & & & & 1.9 & 95 \\
\hline LU9 & 7 & well & 31 & & & 1850 & & & & & 4 & 2.3 & & & 390 & 240 & 200 & 11 & & & & 1.6 & 66 \\
\hline LU9 & 8 & well & 30.5 & 8.6 & & 1860 & 1130 & & & & 5.2 & 0.2 & & & 438 & 241 & 204 & 13 & & 0.48 & & 0.2 & 48 \\
\hline LU13 & 50 & Little Ed Well & 30 & 7.7 & 8.3 & 500 & & 68 & 8.3 & & 25 & 7.3 & & & & 35 & 24 & 1.7 & & & 0.81 & 26.1 & 79 \\
\hline LU15 & 5 & well & 30 & 8 & 8.3 & 1100 & & 230 & 7.3 & & 8 & 2.2 & & & & 170 & 46 & 7 & & & & 1.86 & 41 \\
\hline MKI & 234 & Fort Wingate Well/Santa Fe 'Spring' & 55 & & & 730 & & & & & & & & & 325 & & 7 & & & & & & \\
\hline MK1 & GS64891 & Fort Wingate Well/Santa Fe 'Spring' & 61.11 & & 7.3 & 5520 & & 1100 & 21 & & 264 & 59 & & & 110 & 2990 & 63 & 0.4 & & 1.1 & & & \\
\hline MK2 & 229 & well & 46 & & & 3110 & & & & & & & & & & & & & & & & & \\
\hline MK4 & 431 & well & 42.2 & 8.2 & & 1800 & & & & & & & & & & & & & & & 0.16 & & \\
\hline MK5 & 231 & well & 40.2 & 6.49 & 6.9 & 2850 & 2330 & 370 & 14 & & 260 & 79 & 1 & 4.6 & & 1000 & 240 & 0.4 & 0.52 & 1.1 & 12 & & 22 \\
\hline MK6 & 242 & well & 39 & 8.5 & & 890 & 565 & 190 & & & 11 & 2.6 & & & 240 & 187 & 28 & 0.5 & & & 0.09 & 0.1 & 20 \\
\hline MK7 & 298 & Navajo well & 37.5 & 8.9 & & 703 & & & & & & & & & 200 & 130 & 2 & 3.2 & & & & 0.8 & \\
\hline MK10 & 264 & NTUA \#2 Well & 36 & 8.5 & 8.6 & 500 & & 120 & 0.9 & & 2.4 & 0.1 & & & & 58 & 4.4 & 0.2 & & & 0.02 & 0.09 & 18 \\
\hline MK11 & 261 & well & 35.5 & 8.1 & 8.4 & 450 & & 110 & 1.1 & & 2.4 & 0.2 & & & & 55 & 3.4 & 0.2 & & & 0.02 & 0.49 & 18 \\
\hline MK12 & 297 & Pure Oll Navalo \#1 (Tohachi Well) & 37 & 8.4 & 9.2 & 655 & & 140 & 0.6 & & 1.5 & 0.05 & & & & 130 & 5.8 & 0.4 & & & 0.009 & & 22 \\
\hline MK12 & GS52896 & Pure Oll Navalo \#1 (Tohachi Well) & 35.56 & & 8.3 & 606 & & & & & 5.2 & & & & 212 & 122 & 5.2 & 0.3 & & & & & 22 \\
\hline MK13 & S76NAVAJO & Pure Oil Navajo \#3 Well & 35 & & & & & & & & & & & & & & & & & & & & \\
\hline MK14 & 265 & Mobil Well Crown Point & 33.5 & 8.4 & 8.9 & 415 & & 100 & 1 & & 2.5 & 0.2 & & & & 39 & 3.3 & 0.2 & & 0.06 & 0.11 & & 18 \\
\hline MK15 & 420 & well & 33.5 & 8.6 & & 951 & & & & & & & & & 330 & 240 & 5.7 & & & & & & \\
\hline MK16 & 262 & well & 33 & 7.9 & 8.3 & 450 & & 110 & 1.5 & & 4.7 & 1.2 & & & & 62 & 3.5 & 0.4 & & & \begin{tabular}{|l|}
0.05 \\
\end{tabular} & 0.53 & 19 \\
\hline MK18 & 421 & well & 35.5 & & & 2910 & & & & & & & & & & & 79 & & & & & & \\
\hline MK18 & 423 & well & 32.5 & 8.55 & & 2900 & & & & & & & & & & & & & & & 0.05 & & \\
\hline MK19 & 235 & well & 32 & & & & & & & & 110 & 55 & & & 210 & 560 & 5 & & & & & & \\
\hline MK22 & 202 & well & 30.5 & 7.9 & & 1140 & 730 & 93 & 10 & & 120 & 26 & & & 220 & 400 & 18 & & & 0.5 & 0.01 & 0.25 & \\
\hline MK24 & 260 & well & 30 & 8 & 8.2 & 675 & & 150 & 2.1 & & 12 & 5.1 & & & & 140 & 3.2 & 0.6 & & & 0.06 & 0.53 & 19 \\
\hline OT1 & $45 \mathrm{Ng}$ & $\mathrm{N}-9$ Well & 71.1 & & & & 12500 & & & & 542 & 108 & & & & 820 & 6590 & & & & & 80 & 58 \\
\hline OT2 & $57 N 11$ & $\mathrm{~N}-11$ Well & \begin{tabular}{|l|}
61.1 \\
\end{tabular} & & 6.8 & 14500 & 8980 & & & 2530 & 403 & 80 & & & 240 & 859 & 4060 & 4.7 & & & & & 53 \\
\hline OT3 & 26 & $M-11$ & 33.5 & 7.7 & & 1980 & 1040 & & & & 31 & 5.5 & & & 120 & 85 & 500 & 2.4 & & & & 1.8 & 14 \\
\hline
\end{tabular}




\begin{tabular}{|c|c|c|c|c|c|c|c|c|c|c|c|c|c|c|c|c|c|c|c|c|c|c|c|}
\hline SITEID & SAMPLE & NAME & \begin{tabular}{|c|}
$T M P$ \\
$C$ \\
\end{tabular} & \begin{tabular}{|c|}
$p H$ \\
field
\end{tabular} & $\begin{array}{l}\mathrm{pH} \\
\mathrm{lab}\end{array}$ & $\begin{array}{l}\text { COND } \\
\mathrm{uS} / \mathrm{cm}\end{array}$ & \begin{tabular}{|c|} 
TDS \\
$\mathrm{mg} \Omega$
\end{tabular} & $\begin{array}{c}\mathrm{Na} \\
\mathrm{mg} / \mathrm{L}\end{array}$ & $\begin{array}{c}K \\
m g / L\end{array}$ & $\begin{array}{l}\mathrm{Na+K} \\
\mathrm{mg} / \mathrm{L}\end{array}$ & $\begin{array}{c}\mathrm{Ca} \\
\mathrm{mg} / \mathrm{L}\end{array}$ & \begin{tabular}{c|}
$M g$ \\
$m g /$
\end{tabular} & $\begin{array}{c} \\
m g /\end{array}$ & $\begin{array}{c}\mathrm{Sr} \\
\mathrm{mg} / \mathrm{L}\end{array}$ & $\begin{array}{l}\mathrm{HCO} 3 \\
\mathrm{mg} / \mathrm{L}\end{array}$ & $\begin{array}{l}\mathrm{SO4} \\
\mathrm{mg} / \mathrm{L}\end{array}$ & $\begin{array}{c}\mathrm{Cl} \\
\mathrm{mg} / \mathrm{L}\end{array}$ & $\begin{array}{c}F \\
m g / L\end{array}$ & $\begin{array}{c}B r \\
m g / L\end{array}$ & \begin{tabular}{|c|}
$B$ \\
$m g h$ \\
\end{tabular} & $\begin{array}{c}F a \\
m g / L\end{array}$ & \begin{tabular}{l|}
$\mathrm{NO3}$ \\
$\mathrm{mg} / \mathrm{L}$
\end{tabular} & $\begin{array}{l}\mathrm{S1O2} \\
\mathrm{mgh}\end{array}$ \\
\hline OT3 & 56M11A & M-11(DST 465-504 fi) Well & 50.0 & & 8.00 & 2050 & 1120 & & & 386 & 29 & 4.7 & & & 86 & 133 & 505 & 3.6 & & & & 1.6 & 18 \\
\hline OT3 & $56 \mathrm{M} 118$ & M-11 Well & hot & & 7.7 & 2030 & 1170 & & & 401 & 9.9 & 9.5 & & & 223 & 99 & 445 & 1.6 & & & & 1.2 & 44 \\
\hline OT4 & 64 & FLOUR-1 Well & 33.5 & & 7.4 & 18000 & & 3900 & 18 & & 570 & 210 & & & & 4500 & 4100 & 1.2 & & & & & 29 \\
\hline OT4 & 65 & FLOUR-1 Well & 30 & & 7.5 & 18400 & & 3800 & 17 & & 580 & 220 & 0.46 & 11 & & 4500 & & 1.2 & & 2.3 & 0.024 & & 29 \\
\hline OT5 & S76GART1 & Garton Well & & & & & 7580 & & & & 759 & 288 & & & & 3033 & 3450 & & & & & & 50 \\
\hline OT5 & S76GART2 & Garton Well & & & & & 9111 & & & & 947 & 89 & & & & 3115 & 2880 & & & & & & 36 \\
\hline OT5 & S76GART3 & Garton Well & & & 7.1 & 16000 & 10240 & 2050 & 50 & & & 1652 & & & 70 & 2150 & 4250 & & & & & & \\
\hline OT6 & OLDN8 & N-8 Well & hot (?) & & & & & & & & & & & & & & & & & & & & \\
\hline OT7 & 27 & $\mathrm{~N}-11$ & 61 & 6.8 & & 14500 & 9010 & & & & 400 & 80 & & & 240 & 860 & 4100 & 4.7 & & & & & 53 \\
\hline RA1 & GS9877 & Ojo Callente Hot Spring Soda Spring & 35 & & & 3890 & & & & & 28 & 8.7 & & & 2200 & 168 & 232 & 16 & & & & & 60 \\
\hline RA4 & $\mathrm{NB82-4}$ & Ojo Caliente Hot Spring & 43 & 7.8 & & & & 938 & 40 & & 32 & & 3.5 & & & & & & & & & & 65 \\
\hline RAG & GS8978 & Ojo Caliento HS Sodium Sulfate Spring & & & & 3890 & & & & & 28 & 8.7 & & & 2210 & 165 & 245 & 16 & & 4.6 & & & 56 \\
\hline RA7 & 450 & Ojo Caliente Hot Spring Iron Spring & 40 & 6.6 & & 390 & & 890 & & & & & 3.3 & & 207 & & 270 & & 1.4 & 1.5 & & & \\
\hline RA7 & GS15115 & Ojo Caliente Hot Spring Iron Spring & 42.78 & 6.6 & & 3900 & & 890 & & & & & 3.3 & & 2073 & & 270 & & 1.4 & 1.5 & & & 63 \\
\hline RAB & 452 & Stalue Spring & 36 & & & 1740 & & & & & 145 & 59 & & & 698 & 270 & 110 & 1.4 & & & & 0.2 & 22 \\
\hline RA8 & NB82-3 & Statue Spring & 29 & 7 & & & & 187 & 16 & & 21 & & 0.45 & & & & & & & & & & 29 \\
\hline SA9 & VA-114 & Jemez/GRI WC 23-4 at $4800 \mathrm{ft}$ depth & 214.0 & 6.92 & & & & 2880 & 499 & & 64.3 & 3.4 & 37.4 & 2.54 & & & & & & 33.2 & & & \\
\hline SA10 & $5-6-80$ & Jemez/Sulphur Springs Women's Bathhouse & 88.0 & 4.30 & & 12800 & 6850 & 18.9 & 72 & & 131 & 50 & 0.17 & 0.065 & & 6400 & & 5.2 & & 0.2 & & & 168 \\
\hline SA10 & VA-76 & Jemez/Sulphur Springs Women's Bathhouse & 89.0 & $<2.5$ & & & & & & & & & & & & & & & & & & & \\
\hline SA11 & VA-80 & Jemez/Sulphur Springs main fumarole & 88.0 & 4.30 & & 70 & 95.3 & 0.08 & & & & & & & & 4.5 & & & & & & & \\
\hline SA13 & VA-91 & Jemez HS Travertine Mound Spring & 72.3 & 6.47 & & 4540 & & & & & & & & & 715 & 39.8 & 869 & 5.13 & 3.01 & & & & \\
\hline SA15 & VA14 & Jemez/Sulphur Springs unnamed HS & 63.0 & 2.38 & & 5800 & 2490 & 14.6 & 18.7 & & 90.8 & 16.2 & 0.05 & 0.22 & & 2110 & 3.72 & 0.61 & & & & & 230 \\
\hline SA16 & 266 & Jemez Hot Springs & 52 & & & & 2184 & & & & 166 & 9 & & & 791 & 42 & 820 & & & & 1.2 & 5 & 91 \\
\hline SA16 & 268 & Jemez Hot Springs & 31.5 & & & & & & & & & & & & & & & & & & & & 16 \\
\hline SA16 & 270 & Jemez Hot Springs & 72 & 8.7 & & 3250 & & & & & & & & & & & 832 & & & & & & \\
\hline SA16 & 274 & Jemez Hot Springs & 73 & 7.2 & & 3700 & & & & & 137 & 4.4 & & & 750 & 44 & 855 & 7.1 & & 0.006 & & 0.4 & 64 \\
\hline SA16 & NB82-8 & Jemez Hot Springs & 53 & 7.2 & & & & 638 & 78 & & 92 & & 9.1 & & & & & & & & & & 91 \\
\hline SA17 & 393 & Jemez/Sulphur Springs Lemonade Spring & 53 & 2 & & 3760 & & & & & 150 & 73 & & & & 1190 & 65 & 1 & & & 1.8 & 0.3 & 162 \\
\hline SA17 & $5-10-80$ & Jemez/Sulphur Springs Lemonade Spring & 58.0 & 2.30 & & & 3220 & 7.7 & 5.6 & & 168 & 42 & 0.14 & 0.06 & & 2740 & 17 & 0.52 & & 0.03 & & & 238 \\
\hline SA19 & VA-147 & Jemez HS Gazebo Spring & & 7.09 & & 3900 & & & & & & & & & & & 922 & 2.4 & 3.9 & 5.18 & & & 90 \\
\hline SA19 & $\mathrm{VA}-93$ & Jemez HS Gazebo Spring & 46.3 & 6.66 & & 4270 & & & & & & & & & 720 & 45.5 & 926 & 5.18 & 2.86 & & & & \\
\hline SA2O & 245 & Kaseman \#2 Well (Zla Hol Well) & 32 & & & & 11200 & & & & 400 & 73 & & & 1500 & 3700 & 2700 & & & & 2.3 & & 18 \\
\hline SA2O & 249 & Kaseman \#2 Well (Zla Hol Well) & 50 & & & 15400 & & & & & & & & & & & 3100 & & & & & & \\
\hline SA20 & 250 & Kaseman \#2 Well (Zia Hot Well) & 59 & 6.8 & & 15400 & & & & & 370 & 73 & & & 1480 & 3600 & 3000 & 2.6 & & 6.6 & & & 27 \\
\hline SA2O & 253 & Kaseman \#2 Well (Zia Hol Well) & 52 & & & & & & & & & & & 10 & & & & & & 2.6 & 0.85 & & \\
\hline SA20 & 254 & Kaseman \#2 Well (Zia Hol Well) & 51 & & & & & & & & & & & & & & & & & & & & 7 \\
\hline SA2O & NB82-10 & Kaseman \#2 Well (Zia Hol Woll) & 54 & 6.8 & & & & 3700 & 121 & & 341 & & 5.7 & & & & & & & & & & 39 \\
\hline SA20 & VA-149 & Kaseman \#2 Well (Zia Hol Well) & & 6.81 & & 15500 & & & & & & & & & 1429 & 3350 & 3020 & 2.89 & 3.5 & & & & 34 \\
\hline SA21 & VA-92 & Jemez HS Buddhist Spring & \begin{tabular}{|l|}
43.2 \\
\end{tabular} & 6.49 & & 3200 & & & & & & & & & 660 & 35.1 & 617 & 3.66 & 1.85 & & & & \\
\hline SA23 & 279 & Jemez/Soda Dam Hol Spring & 46 & & & & & & & & & & & & & 40 & 1500 & & 0.02 & 14 & & & \\
\hline SA23 & 280 & Jemez/Soda Dam Hol Spring & 46 & & & 6500 & & & & & & & & & & 43 & 1500 & & 0.9 & 13 & & & \\
\hline SA23 & 358 & Jemez/Soda Dam Hot Spring & 76 & 7.7 & & 13000 & & 3200 & 150 & & 22 & 20 & 12 & 0.11 & & 1200 & 1800 & 16 & 8.3 & 15 & 0.03 & 0.04 & 76 \\
\hline SA23 & NB82-7 & Jemez/Soda Dam Hol Spring & 45 & 7.7 & & & & 995 & 211 & & 187 & & 15 & & & & & & & & & & 30 \\
\hline SA23 & VA-146 & Jemez/Soda Dam Hol Spring & & 6.95 & & 6700 & & & & & & & & & 1476 & 35 & 1567 & 3.85 & 4.6 & & & & 46 \\
\hline SA23 & VA-89 & Jemez/Soda Dam Hol Spring & 47.0 & 6.45 & & 6700 & & & & & & & & & 1490 & 35.5 & 1480 & 3.71 & 5.46 & & & & \\
\hline SA23 & VA-99 & Jemez/Soda Dam Hol Spring & \begin{tabular}{|c|}
47.5 \\
\end{tabular} & & & 6900 & & & & & & & & & 1455 & 37.5 & 1614 & 3.68 & 6 & 14.1 & & & \\
\hline SA24 & 283 & Jemez/Soda Dam Hot Springs (west spring) & 46 & \begin{tabular}{|c|}
6.8 \\
\end{tabular} & & 5780 & & & & & & & & & & & 1490 & & & & & & \\
\hline SA24 & 284 & Jemez/Soda Dam Hot Springs (west spring) & 50 & 7 & & 5760 & & & & & & & & & & & 1510 & & & & & & \\
\hline SA25 & 291 & Jemez/Spence Hot Spring & 44 & 7.3 & & 283 & & & & & 8 & 2 & & & 139 & 17 & 11 & 0.8 & & & & & 71 \\
\hline
\end{tabular}




\begin{tabular}{|c|c|c|c|c|c|c|c|c|c|c|c|c|c|c|c|c|c|c|c|c|c|c|c|}
\hline SITEID & SAMPLE & NAME & $\begin{array}{c}T M P \\
C\end{array}$ & $\begin{array}{c}p H \\
\text { field }\end{array}$ & $\begin{array}{l}p H \\
\text { lab }\end{array}$ & $\begin{array}{l}\text { COND } \\
\mathrm{US} / \mathrm{cm}\end{array}$ & $\begin{array}{c}\text { TDS } \\
m g h\end{array}$ & $\begin{array}{c}\mathrm{Na} \\
\mathrm{mg} / \mathrm{L}\end{array}$ & $\begin{array}{c}K \\
m g / L\end{array}$ & $\begin{array}{l}\mathrm{Na}+\mathrm{K} \\
\mathrm{mg} / \mathrm{L}\end{array}$ & $\begin{array}{c}\mathrm{Ca} \\
\mathrm{mgh}\end{array}$ & $\begin{array}{c}M g \\
m g \Omega\end{array}$ & $\begin{array}{c}\Delta \\
m g /\end{array}$ & $\begin{array}{c}S r \\
m g h\end{array}$ & $\begin{array}{l}\mathrm{HCO} 3 \\
\mathrm{mg} /\end{array}$ & $\begin{array}{l}\mathrm{SO4} \\
\mathrm{mgh}\end{array}$ & $\begin{array}{c}\mathrm{Cl} \\
\mathrm{mg} / \mathrm{L}\end{array}$ & \begin{tabular}{c|}
$F$ \\
$m g h$ \\
\end{tabular} & \begin{tabular}{c|}
$B r$ \\
$m g / 2$
\end{tabular} & $\begin{array}{c}B \\
m g / 2\end{array}$ & \begin{tabular}{c|}
$\mathrm{Fe}$ \\
$\mathrm{mgh}$
\end{tabular} & \begin{tabular}{|l|} 
NO3 \\
mg/h
\end{tabular} & $\begin{array}{l}\mathrm{SIO2} \\
\mathrm{mg} / \mathrm{L}\end{array}$ \\
\hline SA25 & 292 & Jemez/Spence Hot Spring & 39.5 & & & 276 & & & & & & & & & & & & & & & & & \\
\hline SA25 & 293 & Jemez/Spence Hot Spring & 41 & & & 282 & 224 & 55 & 1.8 & & 6 & 2 & 0.69 & 0.03 & 144 & 18 & 12 & 0.7 & 0.1 & 0.07 & & & \\
\hline SA25 & 294 & Jemez/Spence Hot Spring & 39.5 & & & 295 & & & & & & & & & 148 & & & & & & & & \\
\hline SA25 & NB82-6 & Jemez/Spence Hot Spring & 42 & 7.6 & & & & 61 & 1.5 & & 17 & & 0.76 & & & & & & & & & & 69 \\
\hline SA25 & VA-105 & Jemez'Spence Hot Spring & 42.5 & & & 315 & 161 & & & & & & & & 134 & 18.4 & 7.91 & 0.8 & 0.15 & 0.02 & & & \\
\hline SA25 & VA-83 & Jemez/Spence Hot Spring & 41.5 & 7.87 & & 275 & 161 & & & & & & & & 135 & 18 & 7.2 & 0.9 & \begin{tabular}{|l|}
0.11 \\
\end{tabular} & & & & \\
\hline SA26 & 387 & Jemez/Sulphur Springs Men's Bathhouse & 43.5 & & & & 7887 & & & & 303 & 33 & & & & 6156 & 54 & & & & & & 324 \\
\hline SA26 & 389 & Jemez/Sulphur Springs Men's Bathhouse & 87 & 1.8 & & 13800 & & 24 & 31 & & 7 & 10 & 0.07 & & & 35100 & 24 & 1.2 & & & 11.5 & & 190 \\
\hline SA26 & 391 & Jemez/Sulphur Springs Men's Bathhouse & 81 & 1.4 & & 17300 & & 25 & 34 & & 72 & 18 & & & & 4500 & 20 & 0.9 & & 0.1 & 76 & & 180 \\
\hline SA26 & 392 & Jemez/Sulphur Springs Men's Bathhouse & 70 & 2 & & & & 9 & 11 & & 13 & 1 & & & & 676 & 5 & & & & & & \\
\hline SA26 & $5-7-80$ & Jemez/Sulphur Springs Men's Bathhouse & 82.0 & 2.00 & & 10300 & 2597 & 6 & 35 & & 10 & 6.5 & 0.04 & 0.113 & & 2500 & & & & 0.1 & & & 246 \\
\hline SA26 & VA-13 & Jemez/Sulphur Springs Men's Bathhouse & 78.0 & 2.52 & & 4050 & 822 & 2.1 & 8.2 & & 2.1 & 1.25 & 0.02 & 0.03 & & 786 & 2.48 & 6.36 & & & & & 221 \\
\hline SA26 & VA-75 & Jemez/Sulphur Springs Men's Bathhouse & 72.0 & $<2.5$ & & & & & & & & & & & & & & & & & & & \\
\hline SA26 & VA-81 & Jemez/Sulphur Springs Men's Bathhouse & 73.6 & 2.88 & & 1300 & 5.51 & 0.08 & & & & & & & & 2.2 & & 0.59 & & & & & \\
\hline SA27 & 238 & Jemez Pueblo Indian Hot Spring & 35 & 8 & & 5680 & & & & 1240 & 100 & 8.6 & & & 1280 & 286 & 1140 & 7.3 & & 6.1 & & 0.3 & 48 \\
\hline SA28 & 285 & JemezMcCauley Hot Spring & 43 & 8.1 & & 198 & & & & & 11 & 4 & & & 87 & 8 & 8 & 1.6 & & & & & 53 \\
\hline SA28 & 286 & JemezMoCauley Hol Spring & 31.5 & & & 165 & & & & & & & & & & & & & & & & & \\
\hline SA28 & VA-87 & JemezMcCauley Hol Spring & 31.5 & 7.87 & & 190 & & & & & & & & & 80.5 & 6.6 & 3.2 & 1.31 & & & & & \\
\hline SA29 & NB82-5 & Jemez/San Antonio Hot Spring & 42 & 7.4 & & & & 23 & 1.8 & & 5 & & 0.05 & & & & & & & & & & 80 \\
\hline SA30 & 399 & Jemez/Sulphur Springs Footbath Spring & 40.5 & 1.9 & & 4370 & 1730 & 13 & & & 45 & 12 & & & & 1440 & & & & & 92 & 0.7 & 174 \\
\hline SA30 & S-4-80 & Jemez/Sulphur Springs Footbath Spring & 33.0 & 1.10 & & 30200 & 8310 & 10.8 & 94 & & 56 & 26.5 & 0.1 & 0.098 & & 7900 & & 10.6 & & 0.2 & & & 214 \\
\hline SA31 & 434 & Jemez/San Antonio Warm Springs & 38.5 & 6.7 & & 167 & & & & & 7 & 1 & & & 77 & 15 & 17 & 1.6 & & & & & 103 \\
\hline SA34 & 396 & Jemez/Sulphur Springs Electric Spring & 39 & 1.4 & & 12700 & 3160 & 9.6 & 42 & & 101 & 23 & & & & 3820 & 2.5 & 1 & & & 81 & & 206 \\
\hline SA34 & S-5-80 & Jemez/Sulphur Springs Electric Spring & 36.0 & 1.50 & & 12800 & 4580 & 8.5 & 66 & & 114 & 23 & 0.06 & 0.14 & & 4100 & & 5.2 & & & & & 228 \\
\hline SA37 & VA-90 & Jemez/Soda Dam Hidden Warm Spring & 32.0 & 6.20 & & 6000 & & & & & & & & & 1370 & 48.3 & 1240 & 3.31 & 4.11 & & & & \\
\hline SA42 & NB82-9 & San Ysidro Warm Spring & 24 & 7.6 & & & & 1780 & 81 & & 323 & & 5.8 & & & & & & & & & & 18 \\
\hline SA42 & VA-148 & San Ysidro Warm Spring & & 6.50 & & 10000 & & & & & & & & & 1961 & 1181 & 1862 & 4.52 & 4.3 & & & & 20 \\
\hline SFI & 401 & Guaje \#3 Well & 30.5 & 8.1 & & 192 & & & & & 13 & 1.4 & & & 114 & 3.7 & 3 & 0.4 & & & 0.02 & 1 & 52 \\
\hline SF2 & 359 & Los Alamos \#1B Well & 30.5 & 7.8 & & 743 & 522 & 170 & 3.3 & & & & & & 400 & 46 & 16 & 2.8 & & & & 2.8 & \\
\hline SF2 & 361 & Los Alamos \#1B Well & 31.5 & 7.8 & & 778 & & 188 & & & & & & & 437 & & 13 & 2.6 & & & & 2.6 & \\
\hline SF2 & 367 & Los Alamos \#1B Well & 30.5 & 7.9 & & 775 & & 180 & & & & & & & 428 & & 15 & 2.7 & & & & 0.9 & \\
\hline SF2 & 368 & Los Alamos \#1B Well & 32 & 7.8 & & 876 & & 210 & 3.4 & & & & & & 489 & 51 & 16 & 2.7 & & & & 0.8 & 42 \\
\hline SF4 & 301 & Los Alamos \#6 Well & 30 & 8.5 & & 504 & & & & & 3.1 & 0.2 & & & 275 & 12 & 6.5 & 2.4 & & & & 1.9 & 38 \\
\hline SF4 & 305 & Los Alamos \#6 Well & 30 & & & 513 & & & & & & & & & 281 & & 9 & 2.8 & & & & 0.7 & \\
\hline SF4 & 326 & Los Alamos \#6 Well & 30 & 8.6 & & 517 & & 124 & & & & & & & 310 & & 7 & 2 & & & & 1.8 & \\
\hline $\mathrm{SF} 4$ & 349 & Los Alamos \#6 Well & 30 & 8.5 & & 529 & & 128 & & & & & & & 297 & & 11 & 2.6 & & & & 1.8 & \\
\hline $\mathrm{SF}_{4}$ & 355 & Los Alamos \#6 Well & 30.5 & 8.4 & & 635 & & 159 & 1.4 & & & & & & 372 & 18 & $\overline{7.4}$ & 2.4 & & & & 1.9 & 41 \\
\hline SI1 & 118 & Torc well & 45.5 & & & 4380 & & & & & 150 & 18 & & & 210 & 74 & 1300 & 2.6 & & & 0.07 & & \\
\hline$S \mid 2$ & NB82-19 & Torc artesian well & 45 & 7.6 & & & & 864 & 65 & & 148 & & 1.3 & & & & & & & & & & 42 \\
\hline $\mathrm{S} / 4$ & 112 & Torc well & 44.5 & & & 4400 & & & & & 160 & 17 & & & 210 & 73 & 1300 & 3.2 & & & 0.42 & & \\
\hline SI5 & NB82-18 & Torc Sierra Grande Bath & 44 & \begin{tabular}{|l|}
7.6 \\
\end{tabular} & & & & 853 & 64 & & 152 & & 1.3 & & & & & & & & & & 43 \\
\hline SI6 & GS15117 & TorC Yucca Lodge well $14 \mathrm{ft}$ & & 6.8 & & 4500 & & 740 & & & & & 1.1 & & 213 & & 1400 & & 1.9 & 0.3 & & & 41 \\
\hline SI6 & GS18840 & TorC Yucca Lodge well $14 \mathrm{ft}$ & 43.33 & & 7.2 & 4430 & & & & & 174 & 25 & & & 221 & 98 & 1240 & 2.8 & & & & & 39 \\
\hline S16 & GS27039 & TorC Yucca Lodge well $14 \mathrm{ft}$ & & & & 4420 & & & & & 156 & 20 & & & 220 & 95 & 1290 & & & & & & \\
\hline SI6 & GS31092 & TorC Yucca Lodge well $14 \mathrm{ft}$ & 43.33 & & 7.4 & 4450 & & & & & & & & & 221 & 91 & 1300 & & & & & & \\
\hline S16 & GS33929 & TorC Yucca Lodge well $14 \mathrm{ft}$ & 43.61 & & 7.3 & 4450 & & & & & & & & & 216 & & 1290 & & & & & & \\
\hline$S 16$ & GS38827 & TorC Yucca Lodge well $14 \mathrm{ft}$ & 42.22 & & 7.2 & 4400 & & & & & & & & & 228 & $=$ & 1280 & & & & & & \\
\hline SI6 & GS38930 & TorC Yucca Lodge well $14 \mathrm{ft}$ & 40 & & 7.2 & 4460 & & & & & & & & & 227 & & 1280 & & & & & & \\
\hline SI6 & GS43047 & TorC Yucca Lodge well $14 \mathrm{ft}$ & 41.67 & & 7.2 & 4450 & & & & & & & & & 221 & & 1290 & & & & & & \\
\hline
\end{tabular}




\begin{tabular}{|c|c|c|c|c|c|c|c|c|c|c|c|c|c|c|c|c|c|c|c|c|c|c|c|}
\hline SITEID & SAMPLE & NAME & \begin{tabular}{|c|}
$T M P$ \\
$C$ \\
\end{tabular} & \begin{tabular}{|c|}
$p H$ \\
field \\
\end{tabular} & $\begin{array}{l}\mathrm{pH} \\
\text { Jab } \\
\end{array}$ & $\begin{array}{l}\text { COND } \\
U S / \mathrm{cm} \\
\end{array}$ & \begin{tabular}{|c|} 
TDS \\
$m g / L$ \\
\end{tabular} & $\begin{array}{c}\mathrm{Na} \\
\mathrm{mg} / \mathrm{L}\end{array}$ & \begin{tabular}{|c|}
$K$ \\
$m g /$ \\
\end{tabular} & $\begin{array}{l}\mathrm{Na}+\mathrm{K} \\
\mathrm{mg} / \mathrm{L}\end{array}$ & $\begin{array}{c}\mathrm{Ca} \\
\mathrm{mg} / \mathrm{L}\end{array}$ & \begin{tabular}{|c|}
$M g$ \\
$m g /$
\end{tabular} & \begin{tabular}{c|}
$\begin{array}{c}1 \\
m g / 2\end{array}$ \\
\end{tabular} & $\begin{array}{c}s r \\
m g / L\end{array}$ & $\begin{array}{l}\mathrm{HCO} 3 \\
\mathrm{mg} / \mathrm{L}\end{array}$ & $\begin{array}{l}\mathrm{so4} \\
\mathrm{mgh}\end{array}$ & $\begin{array}{c}\mathrm{Cl} \\
\mathrm{mgh}\end{array}$ & \begin{tabular}{|c|}
$\boldsymbol{F}$ \\
$m g /$ \\
\end{tabular} & \begin{tabular}{|c|}
$B r$ \\
$m g / 2$ \\
\end{tabular} & \begin{tabular}{|c|}
$B$ \\
mgh \\
\end{tabular} & \begin{tabular}{c|}
$F e$ \\
$\dot{m} g h$ \\
\end{tabular} & $\begin{array}{l}\mathrm{NO3} \\
\mathrm{mg} / \mathrm{L}\end{array}$ & $\begin{array}{l}\mathrm{SIO2} \\
\mathrm{mg/L}\end{array}$ \\
\hline 516 & GS44734 & TorC Yucca Lodge well $14 \mathrm{ft}$ & 40 & & 7.5 & 4450 & & & & & & & & & 220 & 96 & 1300 & & & & & & \\
\hline $\mathrm{S} 16$ & GS50301 & TorC Yucca Lodge well $14 \mathrm{ft}$ & 41.94 & & 7.2 & 4480 & & & & & & & & & 220 & & 1310 & & & & & & \\
\hline 516 & GS52617 & TorC Yucca Lodge well $14 \mathrm{ft}$ & 42.22 & & 7.2 & 4490 & & & & & & & & & 222 & & 1300 & & & & & & \\
\hline$S 16$ & GS54863 & TorC Yucca Lodge well $14 \mathrm{ft}$ & 41.67 & & 7.8 & 4520 & & & & & & & & & 222 & & 1280 & & & & & & \\
\hline SI6 & GS653 & TorC Yucca Lodge well $14 \mathrm{ft}$ & 43 & & & & & & & & & & & & & & 1285 & & & & & & \\
\hline $\mathrm{SI6}$ & S76W14-65 & TorC Yucca Lodge well $14 \mathrm{ft}$ & 40.83 & & 8.17 & 4300 & 2621 & 786 & 65 & & 121 & 15.5 & 1.01 & & 111 & & 1400 & 2.93 & 1.13 & \begin{tabular}{|l|l|}
0.56 \\
\end{tabular} & & & 44.9 \\
\hline S16 & T41-YLW14 & Torc Yucca Lodge well $14 \mathrm{ft}$ & 43.33 & & & & & & & & 154 & 19 & & & 218 & 86 & 1290 & 3.2 & & & & & \\
\hline $\mathrm{S} 17$ & NB82-17 & TorC Yucca Lodge & 43 & 7.2 & & & & 746 & 65 & & 165 & & 1.2 & & & & & & & & & & 41 \\
\hline$S 18$ & S76YUCCA & TorC Yueca Lodge outdoor pool & 41.67 & & & & & 828 & 64 & & 160 & 15.6 & 1.11 & & & & & & & & & & \\
\hline S112 & 111 & TorC Old Government Spring & 40 & & & 4480 & & & & 748 & 158 & 22 & & & 225 & 89 & 1300 & & & & & 1 & \\
\hline SI12 & GS684 & TorC Old Government Spring & & & & 4590 & & & & & 168 & 36 & & & 217 & 95 & 1314 & & & & & & \\
\hline S113 & 107 & Torc woll & 40 & & & 4410 & & & $\div$ & 760 & 150 & 19 & & & 210 & 76 & 1300 & 3.2 & & & & 2.7 & \\
\hline S115 & 96 & TorC Ponce De Leon Spring & 40 & & & 4480 & & & & & & & & & 220 & & 1300 & & & & & & \\
\hline SI15 & 97 & TorC Ponce De Leon Spring & 40 & & & 4470 & & & & & 160 & 20 & & & 220 & 78 & 1300 & 3.1 & & & & 5.7 & 45 \\
\hline SI15 & 99 & TorC Ponce De Leon Spring & 42 & & & 4470 & & & & 750 & 150 & 21 & & & 210 & 72 & 1300 & 1.7 & & & & 3.3 & 42 \\
\hline SI15 & 100 & TorC Ponce De Leon Spring & 40 & & & 4350 & & & & & 150 & 16 & & & 210 & 75 & 1200 & 2.8 & & & 0.08 & & \\
\hline SI15 & 102 & TorC Ponce De Leon Spring & 40 & 7.2 & & 4460 & & & & & & & & & 230 & & 1300 & & & & & & \\
\hline SI15 & 103 & TorC Ponce Do Leon Spring & 41.5 & 7.2 & & 4480 & & & & & & & & & 220 & & 1300 & & & & & & \\
\hline SI15 & 104 & TorC Ponce De Leon Spring & 40 & 6.8 & & 4500 & & 740 & & & & & 1.1 & & 213 & & 1400 & & 1.9 & 0.3 & & & \\
\hline SI15 & 105 & TorC Ponce De Leon Spring & 42 & 7.2 & & 4400 & & & & & & & & & 230 & & 1300 & & & & & & \\
\hline SI16 & GS3932 & TorC Geronimo (State) Springs & & & & & & & & & & & & & 224 & 90 & 1350 & & & & & & \\
\hline SI17 & NB82-24 & Hillsboro Warm Spring & 34 & 6.8 & & & & 186 & 14 & & 10 & & 0.28 & & & & & & & & & & 135 \\
\hline S119 & 124 & Sun Oil Test Well & 34 & 7.2 & & 2600 & & & & & & & & & 136 & 1660 & 22 & & & & & & \\
\hline $\mathrm{S} 120$ & 88 & Derry Warm Springs & 34 & & & 1660 & & & & & 48 & 20 & & & 372 & 306 & 141 & 6 & & & & 1.3 & 32 \\
\hline S120 & 89 & Derry Warm Springs & 34 & 7.4 & & 1660 & & & & & & & & & 368 & 303 & 158 & & & & & & \\
\hline 5120 & CON54DWS & Derry Warm Springs & 33.89 & & 7.5 & 1650 & 1030 & & & & 52 & 19 & & & 370 & 309 & 160 & 5.8 & & & & & \\
\hline S120 & GS18725 & Derry Warm Springs & 34 & & & 1660 & & & & & 48 & 20 & & & 372 & 306 & 141 & 6 & & & & & 32 \\
\hline 5120 & GS35977 & Derry Warm Springs & 33.89 & & 7.4 & 1660 & 823 & & & & & & & & 368 & 303 & 158 & & & & & & \\
\hline$S \mid 21$ & 90 & well & 34 & & & 1650 & & & & & 52 & 19 & & & 370 & 309 & 160 & 5.8 & & & & 2 & \\
\hline SI21 & 91 & well & 34 & & & 1660 & & & & & 48 & 20 & & & 372 & 306 & 141 & 6 & & & & 1.3 & 32 \\
\hline S121 & 125 & well & 34 & & & 1660 & & & & & 48 & 20 & & & 372 & 306 & 141 & 6 & & & & 1.3 & 32 \\
\hline S124 & HAREMIT & Torc "warm spring" & & & & & 2635 & 381 & 29 & & 156 & 18 & & & 175 & 60 & 1225 & & & & & & 45 \\
\hline$S \mid 25$ & GS44137 & Barney lorio \#1 Fee & & & 8.50 & 5600 & & 1180 & 4.4 & & & & & & 34 & 2860 & 262 & 6.8 & & 3.9 & & & 42 \\
\hline $5 \sqrt{3}$ & 448 & Navajo well & 51.8 & 7.88 & 8.3 & 1200 & & 240 & 2.3 & & 27 & 0.42 & 0.07 & 1.1 & & 430 & 19 & 1.2 & 0.1 & & 0.14 & & 35 \\
\hline SJ5 & 474 & ARCOWS-2 well & 39.9 & 7.52 & 7.6 & 8000 & & 1700 & 18 & & 50 & 28 & 0.84 & 6.7 & & 3800 & 210 & 1.6 & 0.38 & & 0.3 & & 33 \\
\hline S.6 & 444 & Navajo well & 35.5 & & & 2320 & & & & & 4.5 & 2.1 & & & 480 & 480 & 200 & 2.6 & & & & 0.8 & 20 \\
\hline SJ7 & 464 & Navajo 12T-630 Well & 33 & 7.6 & 7.8 & 6000 & & 1400 & 19 & & 78 & 31 & 0.54 & 10 & & 3200 & 190 & 2.1 & 0.2 & & 0.99 & & 16 \\
\hline SJ7 & 466 & Navajo 12T-630 Well & 31 & 7.8 & 7.7 & 4930 & & 890 & 10 & & 160 & 15 & & 9 & & 2500 & 120 & 2.1 & & 0.3 & 0.47 & & 14 \\
\hline SJ8 & 438 & well & 32.8 & 8.21 & & 2720 & & & & & & & & & & & & & & & 0.25 & & \\
\hline SJ9 & 471 & Navajo well & 30.5 & & & 5050 & & & & & 190 & 36 & & & 130 & 2500 & 63 & 1.7 & & & & 0.2 & 17 \\
\hline SJ9 & 473 & Navajo well & 32 & & & 4110 & & & & 850 & 140 & 25 & & & 80 & 2000 & 70 & 1.8 & & & & 0.3 & 17 \\
\hline SJ10 & 467 & Navajo $12 \mathrm{~T}-520 \mathrm{Well}$ & 30 & 7.8 & & 4050 & & & & & 120 & 16 & & & 78 & 2000 & 59 & 2.2 & & & 0.02 & 0.1 & 14 \\
\hline SJ10 & 468 & Navajo 12T-520 Well & 31 & 8 & 8 & 4000 & & 800 & 7.7 & & 58 & 14 & 0.3 & 12 & & 1600 & 110 & 2 & 0.13 & & 0.17 & & 14 \\
\hline$S J 10$ & 470 & Navajo12T-520 Well & 31.1 & 8.03 & 8.1 & 4300 & & 810 & 7.2 & & 110 & 14 & & 11 & & 2100 & 57 & 1.9 & & 0.15 & 0.17 & & 14 \\
\hline SJ11 & 453 & well & 30.5 & $\begin{array}{ll}7.7 \\
\end{array}$ & & 8610 & & & & & & & & & 290 & & 370 & 2 & & & & & \\
\hline SJ12 & 463 & Navajo 12T-629 Well & 30.5 & 8.03 & 8 & 4200 & & 690 & 8.2 & & 160 & 23 & & 9.8 & & 2000 & 83 & 2 & & 0.15 & 0.24 & & 16 \\
\hline SM1 & GS2083 & Montezuma Hot Spring \#1 & & & & 878 & 554 & & & & 14 & 8.3 & & & & 66 & 154 & & & & & & \\
\hline SMT & GS4801 & Montezuma Hot Spring \#1 & & & & 870 & 537 & & & & 8.5 & 0.7 & & & 92 & 49 & 158 & & & & & & \\
\hline SM1 & GS5233 & Monlezuma Hot Spring \#1 & & & & 878 & 531 & & & & & & & & 82 & & 160 & & & & & & \\
\hline
\end{tabular}




\begin{tabular}{|c|c|c|c|c|c|c|c|c|c|c|c|c|c|c|c|c|c|c|c|c|c|c|c|}
\hline SITEID & SAMPLE & NAME & $\begin{array}{c}\text { TMP } \\
C \\
\end{array}$ & $\begin{array}{l}p H \\
\text { fleld } \\
\end{array}$ & \begin{tabular}{|l|}
$p H$ \\
lab \\
\end{tabular} & $\begin{array}{l}\text { COND } \\
\mathrm{uS} / \mathrm{cm} \\
\end{array}$ & $\begin{array}{l}\pi D S \\
m g h \\
\end{array}$ & $\begin{array}{c}N a \\
m g / L\end{array}$ & $\begin{array}{c}K \\
m g / 2\end{array}$ & $\begin{array}{l}\mathrm{Na+K} \\
\mathrm{mgh} \\
\end{array}$ & $\begin{array}{c}\mathrm{Ca} \\
\mathrm{mg} / \mathrm{L}\end{array}$ & $\begin{array}{c}\mathrm{Mg} \\
\mathrm{mg} / \mathrm{R}\end{array}$ & $\begin{array}{c}\mathrm{U} \\
\mathrm{mg} \mathbf{L} \\
\end{array}$ & $\begin{array}{c}\mathbf{S r} \\
\mathrm{mgh}\end{array}$ & $\begin{array}{l}\mathrm{HCO} 3 \\
\mathrm{mg} / \mathrm{L}\end{array}$ & $\begin{array}{l}\mathrm{sO4} \\
\mathrm{mgh}\end{array}$ & $\begin{array}{c}C l \\
m g /\end{array}$ & $\begin{array}{c}\mathbf{F} \\
\mathbf{m g} / \mathbf{L}\end{array}$ & \begin{tabular}{|c|}
$B r$ \\
$m g / 2$
\end{tabular} & \begin{tabular}{|c|}
$B$ \\
$m g /$ \\
\end{tabular} & \begin{tabular}{|c|}
$F e$ \\
$m g /$ \\
\end{tabular} & \begin{tabular}{|l|}
$\mathrm{NO3}$ \\
$\mathrm{mgh}$ \\
\end{tabular} & $\begin{array}{l}102 \\
m g h\end{array}$ \\
\hline SM1 & S76MNTZ1 & Monfezuma Hot Spring \#1 & 55.17 & & 8.38 & 810 & & 200 & 6.9 & & 5 & & 0.36 & & 82 & & 160 & 3.4 & 3.6 & 0.44 & & & 80 \\
\hline SM2 & GS18609 & Monlezuma Hol Spring \#6 & 50.56 & & 9 & 876 & 530 & & & & 4.5 & 1.1 & & & 66 & 42 & 155 & 20 & & & & & 59 \\
\hline SM3 & NB82-11 & Montezuma Hot Spring & 49 & 7.7 & & & & 203 & 6.3 & & 11 & & 0.41 & & & & & & & & & & 68 \\
\hline SM4 & GS18610 & Montezuma Hot Spring \#13 & 41.11 & & 8.8 & 876 & 528 & & & & 4.5 & 1 & & & 77 & 42 & 155 & 20 & & & & & 68 \\
\hline SM6 & S76MNT216 & Montezuma Hot Spring \#16 & & & & & & 192 & 7.8 & & 8 & 0.1 & 0.4 & & & & & & & & & & \\
\hline SM9 & S76MNTZ2 & Monfezuma Hot Spring \#2 & & & & & & 169 & 7.4 & & 5 & 0.1 & 0.39 & & & & & & & & & & \\
\hline SM10 & S76MNTZ20 & Montezuma Hot Spring \#20 & & & & & & 176 & 7.2 & & 6 & 0.1 & 0.42 & & & & & & & & & & \\
\hline SM15 & S76MNTZ15 & Montezuma Hot Spring \#15 & & & & & & 175 & 7.5 & & 4 & 0.1 & 0.39 & & & & & & & & & & \\
\hline SM18 & S76MNTZ18 & Montezuma Hol Spring \#18 & & & & & & 180 & 8.1 & & 10 & 0.3 & 0.4 & & & & & & & & & & \\
\hline SM19 & S76MNTZ19 & Montezuma Hol Spring \#19 & & & & & & 184 & 6.9 & & 5 & 0.1 & 0.4 & & & & & & & & & & \\
\hline 504 & NB82-16 & Bosque del Apache Well \#13 & 33 & 7.4 & & & & 810 & 8.4 & & 120 & & 1.1 & & & & & & & & & & 67 \\
\hline SO5 & 128 & warm well & 33 & 7.22 & 7.4 & 4450 & 2870 & 790 & 35 & & 120 & 41 & & & 400 & 550 & 910 & & & 0.89 & & 4.43 & \\
\hline 506 & 159 & Blue Canyon Well & 32.4 & 8.5 & & 380 & & & & 53 & & & & & 140 & 37 & 14 & 0.6 & & 0.76 & & 1 & 26 \\
\hline 506 & 160 & Blue Canyon Well & 31.5 & 7.1 & & 360 & & 57 & & & & & 0.06 & & 157 & & 16 & & 0.5 & \begin{tabular}{|l|}
0.09 \\
\end{tabular} & & & \\
\hline 506 & 161 & Blue Canyon Well & 30 & & & 380 & & & & & & & & & 140 & 37 & 14 & 0.6 & & 0.08 & & & 26 \\
\hline SO6 & BLUE10-65 & Blue Canyon Well & & & 8.62 & 365 & 145 & 55 & 3.3 & & 27 & 4.3 & 0.07 & & 124 & & 20 & & 0.2 & 0.18 & & & $28^{\circ}$ \\
\hline 506 & GS15116 & Blue Canyon Well & & 7.1 & & 360 & & 57 & & & & & 0.06 & & 157 & & 16 & & 0.5 & 0.09 & & & \\
\hline SO6 & GS3372 & Blue Canyon Weil & 33.33 & & 8.5 & 380 & & & & & & & & & 145 & 37 & 14 & 0.6 & & \begin{tabular}{|l|}
0.76 \\
\end{tabular} & & & 26 \\
\hline 506 & HALL4 & Blue Canyon Well & & & 8 & & & & & & 18 & 5 & & & 166 & 32 & 12 & & & & & & \\
\hline 506 & NB82-12 & Blue Canyon Well & 33 & 7.2 & & & & 55 & 3.3 & & 27 & & 0.06 & & & & & & & & & & 28 \\
\hline 507 & 141 & Socorro Gallery Spring & 31.5 & 8.4 & & 362 & & & 3 & & & & & & 160 & 33 & 16 & 0.7 & & 0.06 & & 1.1 & 39 \\
\hline 507 & 142 & Socorro Gallery Spring & 33.5 & 7.8 & & 346 & & & & & 18 & 4.4 & & & 160 & & & & & & & & \\
\hline 507 & 149 & Socorro Gallery Spring & 30 & 8.4 & & 362 & 231 & 55 & 3 & & & & & & 160 & 33 & 16 & 0.7 & & \begin{tabular}{|l|}
0.06 \\
\end{tabular} & & & 39 \\
\hline 507 & 150 & Socorro Gallery Spring & 31 & \begin{tabular}{|l|}
8.1 \\
\end{tabular} & & 370 & & 50 & & & 18 & 5 & & & 160 & 28 & 8 & & & & & & \\
\hline so7 & 151 & Socorro Gallery Spring & 30 & 7.8 & & 356 & & 52 & & & 13 & 5 & & & 160 & 20 & 12 & & & & & & \\
\hline SO7 & 153 & Socorro Gallery Spring & 30.5 & 7.8 & & 346 & & & & & 4.4 & & & & 150 & & & & & & & & 18 \\
\hline SO7 & 155 & Socorro Gallery Spring & 30 & & & 370 & & 79 & 4 & & 11 & 3.6 & & & 110 & 75 & 20 & & & & & & \\
\hline SO7 & 157 & Socorro Gallery Spring & 32.5 & 8.1 & 8.1 & 352 & 224 & 54 & 3.2 & & 18 & 4 & & & & 31 & 13 & 0.7 & & \begin{tabular}{|l|}
0.12 \\
\end{tabular} & & \begin{tabular}{|l|}
1.59 \\
\end{tabular} & 26 \\
\hline 507 & 158 & Socorro Gallery Spring & 33 & 8.2 & & & 234 & & & & 19 & 5 & & & 160 & 30 & 13 & & & & & & \\
\hline SO7 & CLKPRST & Socorro Gallery Spring & & & & & 318 & & & & 42 & 27 & & & 85 & 102 & 38 & & & & & & 33 \\
\hline 507 & GS38854 & Socorro Gallery Spring & & & 8.4 & 362 & & 55 & 3 & & & & & & 160 & 33 & 16 & 0.7 & & \begin{tabular}{|l|}
0.06 \\
\end{tabular} & & & 39 \\
\hline $\mathrm{SO} 7$ & GS410-65 & Socorro Gallery Spring & 33.11 & & 7.8 & 346 & & & & & 18 & 4.4 & & & 155 & & & & & & & & \\
\hline SO7 & HALL1 & Socorro Gallery Spring & 33 & & 8.1 & 370 & & & & & 18 & 5 & & & 163 & 28 & 8 & & & & & & \\
\hline $\mathrm{SO}$ & HALL2 & Socorro Gallery Spring & & & 7.8 & 356 & & & & & 13 & 5 & & & 156 & 20 & 12 & & & & & & \\
\hline $\mathrm{SO}$ & NB82-13 & Socorro Gallery Spring & 32 & 7.2 & & & & 53 & 3 & & 18 & & 0.05 & & & & & & & & & & 26 \\
\hline 507 & NMHSS-SC & Socorro Gallery Spring & & & 8.35 & 330 & 220 & 52.4 & 2.73 & & 22 & 3.1 & & & 156.6 & 29.5 & 13.4 & 0.22 & & & & & \\
\hline 507 & S76FIG27-1 & Socorro Gallery Spring & 32.56 & & 8.4 & 335 & 249 & 56 & 3 & & 18 & 3.9 & 0.06 & & 142 & & 12 & 1.5 & 0.17 & 0.11 & & & 24 \\
\hline 507 & S76FIG27-2 & Socorro Gallery Spring & 33.56 & & 8.54 & 340 & 234 & 53 & 3 & & 18 & 4 & 0.06 & & 129 & & 16 & & 0.38 & 0.03 & & & 21 \\
\hline SO7 & S76FIG27-3 & Socorro Gallery Spring & 32.44 & & 8.44 & 334 & 245 & 56 & 3 & & 14 & 4 & 0.06 & & 127 & & 16 & 1.1 & 0.17 & 0.04 & & & 22 \\
\hline SO7 & S76FIG27-4 & Socorro Gallery Spring & 33.11 & & 8.43 & 339 & 232 & 53 & 3 & & 18 & 3.9 & 0.06 & & 126 & & 16 & 0.77 & 0.27 & 0.05 & & & 26 \\
\hline 507 & S76GS2-48 & Socorro Gallery Spring & & & & 352 & & & & & 20 & 4.7 & & & 165 & 31 & 13 & & & & & & 28 \\
\hline SO7 & SCOFLD1 & Socorro Gallery Spring & & & & 340 & & 55 & 5 & & 19 & 4 & & & 168 & 30 & 14 & & & & & & \\
\hline SO7 & SCOFLD2 & Socorro Gallery Spring & & & & 347 & & & & & 18 & 5 & & & 159 & 30 & 13 & 0.09 & & & & & \\
\hline SO7 & SCOFLD3 & Socorro Gallery Spring & & & & 348 & & & & & 18 & 5 & & & 156 & 30 & 13 & 1 & & & & & \\
\hline SO7 & SETTLING & Socorro Gallery Spring & & & 8.42 & & 232 & 53 & 3 & & 18 & 4 & 0.01 & & 133 & & 16 & 0.55 & 0.2 & 0.07 & & & 18 \\
\hline SO7 & WALD1956 & Socorro Gallery Spring & 32.78 & & 8.2 & & & & & & 19 & 5 & & & 163 & 30 & 13 & & & & & & \\
\hline 508 & 139 & Socorro/Sedilla Gallery Spring & 30 & 8.5 & & 331 & 255 & 53 & 3 & & 18 & 4.2 & & & & 31 & 13 & 0.7 & & 0.12 & & 1.15 & 26 \\
\hline 508 & GS38853 & Socorro/Sedilla Gallery Spring & & & 8.2 & & & 54 & 2.9 & & & & & & 159 & 23 & 14 & 0.8 & & 0.05 & & & 27 \\
\hline 508 & HALL3 & Socorro/Sedilla Gallery Spring & 31.11 & & 8.4 & 370 & & & & & 18 & 5 & & & 154 & 24 & 10 & & & & & & \\
\hline
\end{tabular}




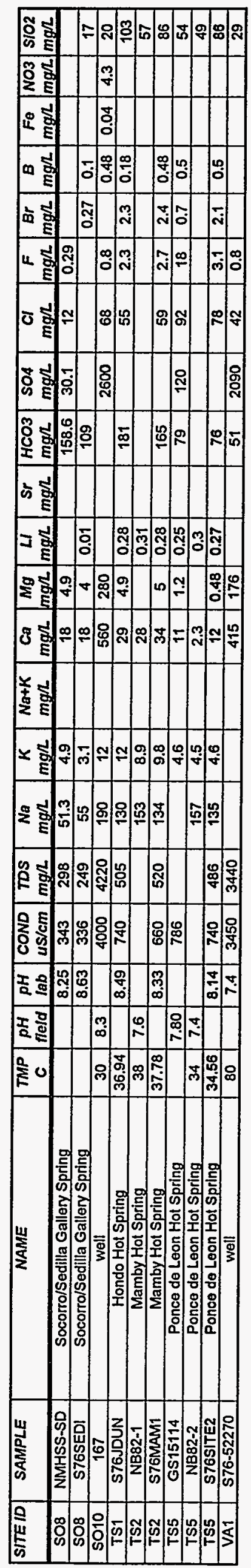




\section{APPENDIX 4}

\section{SITE AND SAMPLE INFORMATION TABLES}

NOTES:

SITE ID

geothermal site number

SAMPLE

cited or assigned sample number or designation

DATE

month/day/yr that sample was taken or reported

NAME

TEMP

well or spring name

temperature ${ }^{\circ} \mathrm{C}$

COND

conductance (uS/cm - microsiemens per centimeter)

TDS

analytical total dissolved solids ( $\mathrm{mg} / \mathrm{L}$ - milligrams per liter)

FLOW

flow rate (L/min - liters per minute)

REFERENCE sample information and chemical analysis source 


\begin{tabular}{|c|c|c|c|c|c|c|c|c|}
\hline SITE ID & SAMPLE & DATE & NAME & $\begin{array}{c}\text { TMP } \\
C \\
\end{array}$ & $\begin{array}{l}\text { COND } \\
\text { uS/cm }\end{array}$ & $\begin{array}{c}\text { TDS } \\
\mathrm{mg} / \mathrm{L}\end{array}$ & $\begin{array}{c}\text { FLOW } \\
\text { L/min }\end{array}$ & REFERENCE \\
\hline$\overline{\text { BE1 }}$ & 219 & $4 / 14 / 75$ & well & 40.5 & 423 & & & WATSTORE 1993 \\
\hline $\mathrm{BE2}$ & JIREC-2 & $? / ? / 83$ & West Mesa \#1 & 32.2 & & 348 & & Jiracek (1983) \\
\hline BE2 & 217 & $4 / 11 / 75$ & well & 34.5 & 402 & & & WATSTORE 1993 \\
\hline BE3 & 222 & $1 / 25 / 83$ & well & 30.5 & 1125 & & & WATSTORE 1993 \\
\hline BE3 & 221 & $1 / 17 / 83$ & well & 32.0 & 1250 & & & WATSTORE 1993 \\
\hline BE3 & 220 & $11 / 13 / 81$ & well & 33.0 & 1160 & & & WATSTORE 1993 \\
\hline BE5 & JIREC-4 & $? / ? / 83$ & West Mesa \#4 & 31.7 & & 356 & & Jiracek (1983) \\
\hline BE5 & GJIR988 & $? / ? / 83$ & West Mesa \#4 & 32.1 & & & & Jiracek (1983) \\
\hline BE6 & 211 & $5 / 9 / 80$ & well & 32.0 & 520 & & 12415 & WATSTORE 1993 \\
\hline BE7 & JIREC-1 & $? / ? / 83$ & Don \#1 & 31.4 & & 420 & & Jiracek (1983) \\
\hline $\mathrm{BE} 8$ & 212 & $4 / 6 / 65$ & well & 31.0 & 495 & 332 & & WATSTORE 1993 \\
\hline BE9 & JIREC-3 & $? / ? / 83$ & West Mesa \#2 & 30.6 & & 330 & & Jiracek (1983) \\
\hline BE10 & 209 & $8 / 25 / 73$ & well & 30 & 524 & 340 & & WATSTORE 1993 \\
\hline BE11 & 223 & $6 / 28 / 72$ & well & 30.0 & 660 & & & WATSTORE 1993 \\
\hline BE11 & 224 & $6 / 28 / 72$ & well & 30.0 & 657 & & & WATSTORE 1993 \\
\hline BE12 & 225 & $6 / 28 / 72$ & well & 30.0 & 456 & & & WATSTORE 1993 \\
\hline BE13 & 227 & $12 / 1 / 78$ & College \#2 & 30.0 & 440 & & 8327 & WATSTORE 1993 \\
\hline CA1 & GILA7 & $? / ? / 75-80$ & hot spring & 64.8 & & 548 & & Swanberg (1980) \\
\hline CA2 & 120 & \begin{tabular}{|l|}
$7 / 24 / 62$ \\
\end{tabular} & hot spring & 60.6 & 767 & 505 & & WATSTORE 1993 \\
\hline CA3 & GS31052 & $12 / 5 / 74$ & Lower Frisco Hot Spring & 35.2 & 1200 & & & Summers (1976) \\
\hline CA3 & LFHS12-74 & $12 / 5 / 74$ & Lower Frisco Hot Spring & 35.22 & 1200 & & & Summers (1976) \\
\hline $\mathrm{CA3}$ & M77-CT2 & $? / ? / 77$ & Lower Frisco Hot Spring & 37.0 & & 1015 & 20 & Mariner and others (1977) \\
\hline $\mathrm{CA3}$ & LFHS66-1 & $2 / 15 / 66$ & Lower Frisco Hot Spring & 37.4 & & & 36.72 & Summers (1976) \\
\hline CA3 & LFHS66-3B & $2 / 15 / 66$ & Lower Frisco Hot Spring & 39.6 & & & 140.06 & Summers (1976) \\
\hline CA3 & LFHS66-4 & $2 / 15 / 66$ & Lower Frisco Hot Spring & 41.2 & & & 3.78 & Summers (1976) \\
\hline CA3 & LFHS66-7 & $2 / 15 / 66$ & Lower Frisco Hot Spring & 45.1 & & & 3.78 & Summers (1976) \\
\hline CA3 & LFHS66-11 & $2 / 15 / 66$ & Lower Frisco Hot Spring & 45.6 & & & & Summers (1976) \\
\hline CA3 & GS42605 & $6 / 11 / 59$ & Lower Frisco Hot Spring & 46.1 & 1780 & 1020 & & Summers (1976) \\
\hline $\mathrm{CA3}$ & LFHS66-8 & $2 / 15 / 66$ & Lower Frisco Hot Spring & 46.9 & & & 3.78 & Summers (1976) \\
\hline CA3 & NB82-34 & $? / ? / 80-82$ & Lower Frisco Hot Spring & 49.0 & & & & Norman and Bernhardt (1982) \\
\hline CA3 & LFHS66-9 & $2 / 15 / 66$ & Lower Frisco Hot Spring & 49.4 & 1940 & & 11.36 & Summers (1976) \\
\hline CA3 & SNM-19 & $6 / 4 / 87$ & Lower Frisco Hot Spring & 50.5 & 1894 & 1142.9 & & Shevenell (1987) \\
\hline CA3 & GS22690 & $6 / 16 / 53$ & Lower Frisco Hot Spring & & 1930 & & 75.71 & Summers (1976) \\
\hline CA3 & GS38864 & $6 / 13 / 58$ & Lower Frisco Hot Spring & & 1660 & & & Summers (1976) \\
\hline$\overline{C A 4}$ & $\sqrt{3}$ & $? / ? / 75-80$ & Lower Frisco Hot Spring & 43.3 & & 992 & & Swanberg (1980) \\
\hline CA4 & SNM-18 & $6 / 4 / 87$ & Lower Frisco Hot Spring & 45.0 & 1495 & 925.8 & & Shevenell (1987) \\
\hline CA4 & J5 & $? / ? / 75-80$ & Lower Frisco Hot Spring & 48.9 & & 1280 & & Swanberg (1980) \\
\hline CA5 & 121 & $6 / 13 / 58$ & Lower Frisco Hot Spring & 43.0 & 1660 & & & WATSTORE 1993 \\
\hline CA6 & $\mathrm{J4}$ & $? / ? / 75-80$ & Lower Frisco Hot Spring & 40.0 & & 768 & & Swanberg (1980) \\
\hline
\end{tabular}




\begin{tabular}{|c|c|c|c|c|c|c|c|c|}
\hline SITE ID & SAMPLE & DATE & NAME & $\begin{array}{c}T M P \\
C \\
\end{array}$ & $\begin{array}{l}\text { COND } \\
\text { uS/cm }\end{array}$ & $\begin{array}{c}\text { TDS } \\
m g / L\end{array}$ & $\begin{array}{c}\text { FLOW } \\
\text { L/min }\end{array}$ & REFERENCE \\
\hline CA7 & 129 & $5 / 22 / 58$ & Upper Frisco Hot Spring & 36.5 & 284 & & & WATSTORE 1993 \\
\hline CA7 & GS38851 & $5 / 22 / 58$ & Upper Frisco Hot Spring & 36.7 & 284 & & & Summers (1976) \\
\hline CA7 & UFHS2-66 & $2 / 18 / 66$ & Upper Frisco Hot Spring & 36.7 & 242 & 223 & 26.12 & Summers (1976) \\
\hline CA7 & $\sqrt{2}$ & $? / ? / 75-80$ & Upper Frisco Hot Spring & 36.7 & & 156 & & Swanberg (1980) \\
\hline CA7 & SNM-20 & $6 / 4 / 87$ & Upper Frisco Hot Spring & 37.0 & 261 & 211.6 & & Shevenell (1987) \\
\hline CA7 & NB82-35 & $? / ? / 80-82$ & Upper Frisco Hot Spring & 39.0 & & & & Norman and Bernhardt (1982) \\
\hline CA8 & MFG3 & $? / ? / 75-80$ & Gila Middle Fork Hot Spring & 36.0 & & 192 & & Swanberg (1980) \\
\hline CA9 & 122 & $12 / 5 / 74$ & Lower Frisco Hot Spring & 35.0 & 1200 & & & WATSTORE 1993 \\
\hline CA10 & 168 & $8 / 4 / 79$ & Pueblo Windmill & 33.8 & & 950 & & WATSTORE 1993 \\
\hline CA10 & DL-32-NM & $8 / ? / 79$ & Pueblo Windmill & 33.8 & & & & Levitte and Gambill (1980) \\
\hline CA10 & 169 & $10 / 30 / 80$ & Pueblo Windmill & 34.0 & 1600 & & 45.42 & WATSTORE 1993 \\
\hline CA10 & MYERS1 & $10 / 30 / 80$ & Pueblo Windmill & 34.0 & 1600 & & & Myers (1992) \\
\hline CA11 & FCS2-66 & $2 / 18 / 66$ & Frieborn Canyon Hot Spring & 33.3 & 150 & 151 & & Summers (1976) \\
\hline CA12 & NNS-BOIL & $2 / 26 / 66$ & hot spring & 32.8 & & & 204.41 & Summers (1976) \\
\hline CA12 & NNS-CAS & $2 / 26 / 66$ & hot spring & 34.4 & & & 204.41 & Summers (1976) \\
\hline CA13 & GS54856 & $7 / 31 / 64$ & test well & 32.2 & 2710 & 2540 & & Summers (1976) \\
\hline CA14 & 130 & $7 / 13 / 79$ & well & 32 & 330 & 256 & & WATSTORE 1993 \\
\hline CA15 & 119 & $7 / 31 / 64$ & well & 32 & 2710 & 2540 & 15.14 & WATSTORE 1993 \\
\hline CA16 & MFG1 & $? / 2 / 75-80$ & Gila Middle Fork Hot Spring & 31.0 & & 196 & & Swanberg (1980) \\
\hline CA17 & MYERS2 & $10 / 29 / 80$ & well & 28.0 & 1440 & & & Myers (1992) \\
\hline CA17 & MYERS3 & $11 / 20 / 80$ & well & 29.0 & 1300 & & & Myers (1992) \\
\hline CA18 & MEAD-10 & $11 / 12 / 69$ & Gila Middle Fork Meadows HS & 27.5 & 215 & 150 & 129 & Summers (1976) \\
\hline CA18 & MEAD-19 & $11 / 12 / 69$ & Gila Middle Fork Meadows HS & 27.5 & 215 & 155 & 129 & Summers (1976) \\
\hline CA18 & MEAD-5 & $11 / 12 / 69$ & Gila Middle Fork Meadows HS & 28.0 & 220 & 160 & 76.5 & Summers (1976) \\
\hline CA18 & MEAD-12 & $11 / 12 / 69$ & Gila Middle Fork Meadows HS & 29.5 & 220 & 150 & 3.6 & Summers (1976) \\
\hline CA18 & MEAD-14 & $11 / 12 / 69$ & Gila Middle Fork Meadows HS & $32: 5$ & 215 & 145 & & Summers (1976) \\
\hline CA19 & MGSEEP & $2 / 26 / 66$ & warm seep & 27.2 & & & & Summers (1976) \\
\hline CA20 & US107 & $? / ? / 75-80$ & Zuni Salt Lake Warm Spring & 26.0 & & 220152 & & Swanberg (1980) \\
\hline CA21 & GS36104 & $6 / 23 / 57$ & Gila Middle Fork Pool HS & 52.2 & 422 & & & Summers (1976) \\
\hline CA21 & NB82-33 & $? / ? / 80-82$ & Gila Middle Fork Pool HS & 65.0 & & & & Norman and Bernhardt (1982) \\
\hline CA21 & PHS-SUM & $2 / 21 / 66$ & Gila Middle Fork Pool HS & 65.3 & 720 & 514 & 178.2 & Summers (1976) \\
\hline $\mathrm{CA21}$ & SNM-14 & $6 / 2 / 87$ & Gila Middle Fork Pool HS & 65.5 & 777 & 580.8 & & Shevenell (1987) \\
\hline CA21 & M77-CT1 & $? / ? / 77$ & Gila Middle Fork Pool HS & 66.0 & & 587 & & Mariner and others (1977) \\
\hline CA21 & GS62058 & $2 / 21 / 66$ & Gila Middle Fork Pool HS & & 771 & 508 & & Summers (1976) \\
\hline CA22 & MFG2 & $? / ? / 75-80$ & Gila Middle Fork Hot Spring & 37.0 & & 188 & & Swanberg (1980) \\
\hline CA23 & MFG4 & $? / ? / 75-80$ & Gila Middle Fork Hot Spring & 26.0 & & 168 & & Swanberg (1980) \\
\hline CA23 & SW307 & $? / ? / 75-80$ & Gila Middle Fork Hot Spring & 32.0 & & 224 & & Swanberg (1980) \\
\hline CA23 & SW306 & $? / ? / 75-80$ & Gila Middle Fork Hot Spring & 34.0 & & 192 & & Swanberg (1980) \\
\hline CB2 & 210 & $7 / 10 / 86$ & well & 52.8 & 7000 & 5450 & & WATSTORE 1993 \\
\hline
\end{tabular}




\begin{tabular}{|c|c|c|c|c|c|c|c|c|}
\hline SITE ID & SAMPLE & DATE & NAME & $\begin{array}{c}T M P \\
C\end{array}$ & $\begin{array}{l}\text { COND } \\
\text { uS/cm }\end{array}$ & $\begin{array}{c}\text { TDS } \\
\mathrm{mg} / \mathrm{L}\end{array}$ & $\begin{array}{l}\text { FLOW } \\
\text { L/min }\end{array}$ & REFERENCE \\
\hline$\overline{\mathrm{CB3}}$ & 183 & $2 / 15 / 89$ & Acoma \#1 Well & 40.5 & 1270 & & & WATSTORE 1993 \\
\hline CB3 & 180 & $9 / 21 / 84$ & Acoma \#1 Well & 41.5 & 1300 & & 1892.5 & WATSTORE 1993 \\
\hline CB3 & 181 & $10 / 4 / 84$ & Acoma \#1 Well & 41.7 & 1350 & & 1797.9 & WATSTORE 1993 \\
\hline CB4 & 170 & $12 / 15 / 86$ & well & 41.0 & 2800 & 2320 & & WATSTORE 1993 \\
\hline C85 & 177 & $12 / 6 / 86$ & well & 38.1 & 3300 & 3180 & & WATSTORE 1993 \\
\hline CB6 & 178 & $5 / 14 / 75$ & well & 36 & 989 & & 261.17 & WATSTORE 1993 \\
\hline CB7 & 184 & $11 / 17 / 86$ & well & 34.5 & 5500 & 5360 & & WATSTORE 1993 \\
\hline CB8 & 171 & $11 / 26 / 86$ & well & 34.0 & 8000 & 6280 & & WATSTORE 1993 \\
\hline CB9 & 197 & $11 / 10 / 64$ & well & 31.5 & 18000 & & & WATSTORE 1993 \\
\hline CB9 & 193 & $10 / 7 / 63$ & well & 34 & 25200 & 18500 & & WATSTORE 1993 \\
\hline CB10 & 179 & $11 / 18 / 86$ & well & 33.1 & 4450 & 3910 & & WATSTORE 1993 \\
\hline CB12 & 226 & $9 / 30 / 75$ & well & 32.0 & 550 & 340 & & WATSTORE 1993 \\
\hline CB13 & LUC-11 & $5 / ? / 80$ & Salado Spring & 25 & 3800 & & & Goff and others (1983) \\
\hline CV1 & 95 & $7 / 23 / 81$ & well & 33.0 & 900 & 651 & & WATSTORE 1993 \\
\hline DA1 & GROSS3 & $? / ? / 81-86$ & Chaffee 55-25 & 68.0 & & & & Gross (1988) \\
\hline DA1 & GROSS4 & $? / ? / 81-86$ & Chaffee 55-25 & 68.0 & & & & Gross (1988) \\
\hline DA1 & CHAF55-25 & $? / ? / 81$ & Chaffee 55-25 & 69.0 & 2300 & 1480 & & Files SWTDI \\
\hline DA2 & SNM-5 & $6 / 1 / 87$ & Radium Hot Springs Bailey Well \#15 & 70.0 & 7050 & 3944.3 & & Shevenell (1987) \\
\hline DA3 & CLARYST & $? / ? / 49$ & Clary and Ruther State 1 & 69.4 & & & & Files SWTDI \\
\hline DA4 & CHF35-25 & $12 / ? / 81$ & Chaffee 35-25 & 68 & 2580 & 1626 & & Icerman and Lohse (1983) \\
\hline DA4 & GROSS1 & $? / ? / 81-86$ & Chaffee $35-25$ & 68.0 & & & & Gross (1988) \\
\hline DA5 & 280LC2 & $2 / 2 / 80$ & LC-2 & 52.5 & 2530 & 2004 & & Files SWTDI \\
\hline DA5 & GROSS8 & $? / ? / 81-86$ & LC-2 & 68.0 & & & & Gross (1988) \\
\hline DA6 & EXXONOLE & $12 / 6 / 83$ & Exxon Beard Ole Federal & 65.5 & & & & Files SWTDI \\
\hline DA7 & CHF12-24 & $1 / 2 / 82$ & Chaffee 12-24 & 65 & 3000 & 1968 & & Icerman and Lohse (1983) \\
\hline DA7 & GROSS2 & $? / ? / 81-86$ & Chaffee 12-24 & 65.0 & & & & Gross (1988) \\
\hline DA8 & GROSS6 & $? / ? / 81-86$ & NMSUPG-3 & 63.0 & & & & Gross (1988) \\
\hline DA8 & 12581PG3 & $1 / 25 / 81$ & NMSU PG-3 & 63.3 & & 1981 & 757.2 & Cunniff and others (1981) \\
\hline DA8 & 1980PG3 & $? / ? / 80$ & NMSU PG-3 & & 3130 & & & Cunniff and others (1984) \\
\hline DA8 & NDPG3 & $? / ? / 80-84$ & NMSU PG-3 & & & 1748 & & Cunniff and others (1984) \\
\hline DA9 & S76BAILEY & $2 / 7 / 66$ & Radium Hot Springs Bailey's bathhouse & 60.8 & 5600 & 3731 & & Summers (1976) \\
\hline DA9 & BTHSE & $7 / 10 / 81$ & Radium Hot Springs Bailey's bathhouse & & & 874 & & Files SWTDI \\
\hline DA9 & NMPHL316 & $5 / 4 / 62$ & Radium Hot Springs Bailey's bathhouse & & 6100 & & & Summers (1976) \\
\hline DA10 & BINNS1 & $? / 2 / 87$ & Certified Sand Well & 58.8 & & & 12.62 & Files SWTDI \\
\hline DA12 & SNM-6 & $6 / 1 / 87$ & Radium Hot Springs Hotel Well \#2 & 43.0 & 6530 & 3728.4 & & Shevenell (1987) \\
\hline DA12 & M77-DA1 & $? / ? / 77$ & Radium Hot Springs Hotel Well \#2 & 52.0 & & 3857 & & Mariner and others (1977) \\
\hline DA12 & 70 & $12 / 4 / 74$ & Radium Hot Springs Hotel Well \#2 & 52.5 & 6100 & & & WATSTORE 1993 \\
\hline DA12 & 71 & $4 / 29 / 57$ & Radium Hot Springs Hotel Well \#2 & 53.0 & 6210 & & & WATSTORE 1993 \\
\hline DA12 & $\mathrm{B} 2$ & $? / ? / 75-80$ & Radium Hot Springs Hotel Well \#2 & 53.0 & & 3532 & & Swanberg (1980) \\
\hline
\end{tabular}




\begin{tabular}{|c|c|c|c|c|c|c|c|c|}
\hline SITE ID & SAMPLE & DATE & NAME & $\begin{array}{c}T M P \\
C\end{array}$ & $\begin{array}{l}\text { COND } \\
\text { uS/cm }\end{array}$ & $\begin{array}{c}\text { TDS } \\
\mathrm{mg} / \mathrm{L}\end{array}$ & $\begin{array}{l}\text { FLOW } \\
\text { Lmin }\end{array}$ & REFERENCE \\
\hline$\overline{\mathrm{DA} 12}$ & NB82-21 & ?/?/80-82 & Radium Hot Springs Hotel Well \#2 & 53.0 & & & & Norman and Bernhardt (1982) \\
\hline DA12 & RHSHOT2 & $12 / 16 / 65$ & Radium Hot Springs Hotel Well \#2 & 60.0 & 5800 & & & Summers (1976) \\
\hline DA12 & S76HRSW2 & $12 / 4 / 74$ & Radium Hot Springs Hotel Well \#2 & 60.0 & & & & Summers (1976) \\
\hline DA12 & 69 & $5 / 17 / 48$ & Radium Hot Springs Hotel Well \#2 & 85.5 & 6060 & & & WATSTORE 1993 \\
\hline DA12 & GS10160 & $5 / 17 / 48$ & Radium Hot Springs Hotel Well \#2 & 85.6 & 6060 & & & Summers (1976) \\
\hline DA12 & GS35979 & $4 / 29 / 57$ & Radium Hot Springs Hotel Well \#2 & & 6210 & & & Summers (1976) \\
\hline DA12 & GS31053 & $12 / 4 / 74$ & Radium Hot Springs Hotel Well \#2 & & 6100 & & & Summers (1976) \\
\hline DA12 & GS38867 & $4 / 24 / 58$ & Radium Hot Springs Hotel Well \#2 & & 5540 & & & Summers (1976) \\
\hline DA12 & S76RSNMS & $? / ? / ?$ & Radium Hot Springs Hotel Well \#2 & & & 3460 & & Summers (1976) \\
\hline DA13 & 280LC1 & $2 / ? / 80$ & LC-1 & 67.9 & 922 & 576 & & Files SWTDI \\
\hline DA14 & SNM-7 & $6 / 1 / 87$ & Radium Hot Springs Roy Smith Well & 50.0 & 5230 & 2978.5 & & Shevenell (1987) \\
\hline DA15 & SNM-9 & $6 / 1 / 87$ & NMSU PG-2 & 43.0 & 3290 & 2232.6 & & Shevenell (1987) \\
\hline DA15 & 4681PG2 & $4 / 6 / 81$ & NMSU PG-2 & 47.7 & & 2070 & & Cunniff (1981) \\
\hline DA15 & NB82-23 & $? / ? / 80-82$ & NMSU PG-2 & 59.0 & & & & Norman and Bernhardt (1982) \\
\hline DA15 & NMSU81479 & $8 / 14 / 79$ & NMSU PG-2 & & 2480 & 1704 & & Files SWTDI \\
\hline DA16 & 11 & $11 / 25 / 61$ & Pure Oil Federal "H" 1 & 45.0 & 7380 & & 1892.5 & WATSTORE 1993 \\
\hline DA16 & GS48858 & $10 / 25 / 61$ & Pure Oil Federal "H" 1 & 45.0 & 7380 & & & Summers (1976) \\
\hline DA17 & NATIONW81 & $12 / 1 / 72$ & Nations Well & 43.0 & 3040 & & & Wilson and others (1981) \\
\hline DA17 & LASALTNA & $2 / 6 / 66$ & Nations Well & 45.1 & 2400 & 1983 & & Summers (1976) \\
\hline DA18 & 47 & $12 / 1 / 72$ & well & 43 & 3040 & & & WATSTORE 1993 \\
\hline DA19 & LASALTHU & $2 / 6 / 66$ & Husand Well & 42.5 & 2000 & 1706 & & Summers (1976) \\
\hline DA20 & 19 & $7 / 17 / 86$ & well & 31.5 & 1580 & 955 & & WATSTORE 1993 \\
\hline DA20 & 18 & $7 / 16 / 86$ & well & 35.0 & 2100 & 1350 & & WATSTORE 1993 \\
\hline DA20 & 17 & $7 / 16 / 86$ & well & 42.5 & 10900 & 6990 & & WATSTORE 1993 \\
\hline DA21 & LASALTRO & $2 / 6 / 66$ & Rowan well & 36.8 & 1500 & 1093 & & Summers (1976) \\
\hline DA22 & 46 & $9 / 8 / 75$ & Tellyer Well & 36.5 & 1020 & & 151.4 & WATSTORE 1993 \\
\hline DA22 & TELLYER1 & $9 / 8 / 75$ & Tellyer Well & 36.5 & 1020 & & & Wilson and others (1981) \\
\hline DA22 & TELLYER2 & $12 / 1 / 72$ & Tellyer Well & 36.5 & 1110 & & & Wilson and others (1981) \\
\hline DA23 & S7623S2W & $2 / 5 / 66$ & Running Indian Well & 35.0 & 2200 & 1450 & & Summers (1976) \\
\hline DA23 & GS28934 & $1 / 11 / 73$ & Running Indian Well & 36.1 & 2310 & & & Summers (1976) \\
\hline DA24 & SD1 & $? / ? / 75-80$ & well & 36.0 & & 2020 & & Swanberg (1980) \\
\hline DA25 & S76NMS112 & $11 / ? / 63$ & well & 35.6 & 1800 & 1020 & & Summers (1976) \\
\hline DA26 & NB82XX & $? / ? / 82$ & NMSU GD-1 & 30.0 & & & & Norman and Bernhardt (1982 \\
\hline DA26 & 1GD1 & $? / ? / 82$ & NMSU GD-1 & 35.0 & 2370 & & 965.4 & Cunniff (1982) \\
\hline DA26 & 2GD1 & $1 / 13 / 61$ & NMSU GD-1 & & & 1550 & & Cunniff (1981) \\
\hline DA26 & 3GD1 & $2 / 1 / 62$ & NMSU GD-1 & & & 1200 & & Cunniff (1981) \\
\hline DA26 & 4GD1 & $1 / 13 / 81$ & NMSU GD-1 & & & & & Cunniff (1981) \\
\hline DA26 & 5GD1 & $2 / 13 / 81$ & NMSU GD-1 & & & & & Cunniff (1981) \\
\hline DA27 & S76LASWH & $2 / 6 / 66$ & White well & 34.5 & 1460 & 1000 & & Summers (1976) \\
\hline
\end{tabular}




\begin{tabular}{|c|c|c|c|c|c|c|c|c|}
\hline SITE ID & SAMPLE & DATE & NAME & $\begin{array}{c}T M P \\
C \\
\end{array}$ & $\begin{array}{l}\text { COND } \\
\text { uS/cm }\end{array}$ & $\begin{array}{c}\text { TDS } \\
m g / L\end{array}$ & $\begin{array}{l}\text { FLOW } \\
\text { L/min }\end{array}$ & REFERENCE \\
\hline DA27 & NPHL870 & $7 / 2 / 65$ & White well & & 1665 & 1020 & & Summers (1976) \\
\hline DA28 & 72 & $7 / 31 / 80$ & well & 34 & 488 & & & WATSTORE 1993 \\
\hline DA29 & 23 & $5 / 6 / 76$ & well & 33.5 & 950 & 554 & & WATSTORE 1993 \\
\hline DA30 & 9 & $7 / 24 / 75$ & well & 30.5 & 890 & & 121.12 & WATSTORE 1993 \\
\hline DA30 & 10 & $7 / 24 / 75$ & well & 33.3 & 490 & & 113.55 & WATSTORE 1993 \\
\hline DA33 & GS28683 & $2 / 1 / 55$ & well & 32.2 & 4640 & 2930 & 49.97 & Summers (1976) \\
\hline DA33 & 44 & $1 / 11 / 73$ & well & 36 & 2310 & & & WATSTORE 1993 \\
\hline DA34 & 43 & $3 / 16 / 55$ & well & 32.0 & 2320 & & 11.36 & WATSTORE 1993 \\
\hline DA36 & 24 & $3 / 28 / 73$ & Berino Well & 31 & 1120 & & & WATSTORE 1993 \\
\hline DA36 & GS29520 & $3 / 28 / 73$ & Berino Well & 31.1 & 1120 & & & Summers (1976) \\
\hline DA36 & S76BERING & $1 / 24 / 73$ & Berino Well & & 1080 & 740 & & Summers (1976) \\
\hline DA37 & SD22 & $? / ? / 75-80$ & Souse Springs & 31.0 & & 312 & & Swanberg (1980) \\
\hline DA39 & 29 & $7 / 28 / 73$ & well & 31 & 778 & & & WATSTORE 1993 \\
\hline DA39 & GS221153 & $7 / 28 / 73$ & well & 31.1 & 778 & & & Summers (1976) \\
\hline DA40 & 21 & $1 / 10 / 78$ & well & 31.0 & 804 & & & WATSTORE 1993 \\
\hline DA41 & 2 & $8 / 29 / 86$ & well & 31.0 & 5700 & 3780 & & WATSTORE 1993 \\
\hline DA42 & 93 & $2 / 5 / 73$ & well & 31 & 2070 & & & WATSTORE 1993 \\
\hline DA43 & COLM1 & $? / ? / 75-80$ & Pol Ranch Windmill & 30.5 & & 512 & & Swanberg (1980) \\
\hline DA44 & 48 & $10 / 22 / 90$ & SC2 & 30.5 & 270 & & & WATSTORE 1993 \\
\hline DA45 & 12 & $8 / 17 / 86$ & well & 30.5 & 2200 & 1300 & & WATSTORE 1993 \\
\hline DA45 & 14 & $8 / 19 / 86$ & well & 30.5 & 1000 & 656 & & WATSTORE 1993 \\
\hline DA45 & 13 & $8 / 19 / 86$ & well & 32 & 600 & 475 & & WATSTORE 1993 \\
\hline DA46 & 52 & $7 / 31 / 81$ & $\mathrm{~T}-18$ & 30.0 & 686 & & 30.28 & WATSTORE 1993 \\
\hline DA46 & 53 & $8 / 10 / 82$ & $T-18$ & 30.0 & 710 & & & WATSTORE 1993 \\
\hline DA47 & 38 & $6 / 19 / 75$ & Dominguez Bros Well & 30 & 3350 & & & WATSTORE 1993 \\
\hline DA47 & 39 & $11 / 1 / 72$ & Dominguez Bros Well & 31.5 & 2870 & & & WATSTORE 1993 \\
\hline DA49 & NMPLH997 & $8 / 16 / 60$ & Railroad Well & & 2270 & & & Summers (1976) \\
\hline DA50 & HUNTWATW & $8 / 26 / 88$ & Radium Springs College Ranch Windmill & & & & & Files SWTDI \\
\hline DA51 & SCOTTRHS & $11 / 17 / 54$ & Radium Hot Springs Hotel Well \#1 & 53.3 & 6100 & 3620 & & Summers (1976) \\
\hline DA51 & GS2053 & $8 / ? / 22$ & Radium Hot Springs Hotel Well \#1 & & & 3738 & & Summers (1976) \\
\hline DA52 & $21 H 0600$ & $3 / 30 / 93$ & Radium Springs Masson 21 & & & & & Files SWTDI \\
\hline DA52 & $21 \mathrm{H} 0900$ & $3 / 30 / 93$ & Radium Springs Masson 21 & & & & & Files SWTDI \\
\hline DA52 & $21 \mathrm{H} 1800$ & $3 / 30 / 93$ & Radium Springs Masson 21 & & & & & Files SWTDI \\
\hline DA52 & 21TRUN & $3 / 30 / 93$ & Radium Springs Masson 21 & & & & & Files SWTDI \\
\hline DA52 & 86MASS21 & $8 / 11 / 86$ & Radium Springs Masson 21 & & & 3682 & & Files SWTDI \\
\hline DA53 & 89MASS22 & $4 / 2 / 89$ & Radium Springs Masson 22 & & & & & Files SWTDI \\
\hline DA53 & SWL1295 & $2 / 1 / 88$ & Radium Springs Masson 22 & & & & & Files SWTDI \\
\hline DA53 & AA03261 & $7 / 16 / 92$ & Radium Springs Masson 23 & & 5960 & & & Files SWTDI \\
\hline DA54 & 87 MASS23 & $4 / 2 / 87$ & Radium Springs Masson 23 & & & & & Files SWTDI \\
\hline
\end{tabular}




\begin{tabular}{|c|c|c|c|c|c|c|c|c|}
\hline SITE ID & SAMPLE & DATE & NAME & $\begin{array}{c}\text { TMP } \\
C \\
\end{array}$ & $\begin{array}{l}\text { COND } \\
\text { uS/cm }\end{array}$ & $\begin{array}{c}\text { TDS } \\
\mathrm{mg} / \mathrm{L}\end{array}$ & $\begin{array}{l}\text { FLOW } \\
\text { Lmin }\end{array}$ & REFERENCE \\
\hline DA54 & 89MASS23 & $4 / 2 / 89$ & Radium Springs Masson 23 & & & & & Files SWTDI \\
\hline DA54 & SWL1296 & $2 / 1 / 88$ & Radium Springs Masson 23 & & & & & Files SWTDI \\
\hline DA54 & SWL5478 & $12 / 13 / 88$ & Radium Springs Masson 23 & & & & & Files SWTDI \\
\hline DA55 & 311801 & $4 / 30 / 93$ & Radium Springs Masson 26 & & & & & Files SWTD! \\
\hline DA55 & AA03262 & $7 / 16 / 92$ & Radium Springs Masson 26 & & 6030 & & & Files SWTDI \\
\hline DA56 & 1RYAN7235 & $12 / 20 / 86$ & Radium Springs Ryan 72-35 & & & 2584 & & Files SWTDI \\
\hline DA57 & GROSS7 & $? / ? / 81-88$ & NMSU GD-2 & 42.0 & & & & Gross (1988) \\
\hline DA57 & JET468 & $9 / 2 / 82$ & NMSU GD-2 & $\cdot$ & 3120 & 1948 & & Cunniff and others (1983) \\
\hline DA57 & JET840 & $9 / 2 / 82$ & NMSU GD-2 & & 2680 & 1787 & & Cunniff and others (1983) \\
\hline DA58 & NMSUOW1 & $12 / 10 / 81$ & NMSU OW-1 & & & 1765 & & Cunniff (1981) \\
\hline DA59 & SNM-8 & $6 / 1 / 87$ & NMSU PG-1 & 58.5 & 3350 & 2273.5 & & Shevenell (1987) \\
\hline DA59 & PG1COMP & $? / 2 / 82$ & NMSU PG-1 & 60.6 & 3110 & & & Cunniff (1982) \\
\hline DA59 & GROSS5 & $? / ? / 81-88$ & NMSU PG-1 & 61.0 & & & & Gross (1988) \\
\hline DA59 & NB82-22 & $? / 2 / 80-82$ & NMSU PG-1 & 61.0 & & & & Norman and Bernhardt (1982) \\
\hline DA59 & N10PG1 & $? / ? / 80-84$ & NMSU PG-1 & & 3720 & 2044 & & Cunniff and others (1984) \\
\hline DA59 & N4PG1 & $? / ? / 80-84$ & NMSU PG-1 & & 6590 & 4220 & & Cunniff and others (1984) \\
\hline DA59 & N7PG1 & $? / ? / 80-84$ & NMSU PG-1 & & 4510 & 2868 & & Cunniff and others (1984) \\
\hline DA59 & N8PG1 & $? / ? / 80-84$ & NMSU PG-1 & & 4050 & 2416 & & Cunniff and others (1984) \\
\hline DA59 & NMSU9430 & $? / ? / 86$ & NMSU PG-1 & & 2800 & 1820 & & Files SWTDI \\
\hline DA59 & PG1PART1 & $10 / 24 / 80$ & NMSU PG-1 & & & 1936 & & Cunniff (1981) \\
\hline DA59 & PGIPART2 & $7 / 21 / 80$ & NMSU PG-1 & & & 1904 & & Cunniff (1981) \\
\hline DA59 & PGIPART3 & $? / 2 / 80$ & NMSU PG-1 & & 3110 & & & Cunniff and others (1984) \\
\hline DA60 & DST1PG4 & $4 / 2 / 86$ & NMSU PG-4 & 63.0 & 2720 & 1695 & & Files SWTDI \\
\hline DA60 & DST4PG4 & $4 / ? / 86$ & NMSU PG-4 & 63.0 & 2790 & 1854 & & Files SWTDI \\
\hline DA60 & BAIL370 & $11 / 284$ & NMSUPG-4 & & 2450 & 1636 & & Cunniff and Chintawong (1985) \\
\hline DA60 & DRILL13 & $10 / ? / 84$ & NMSU PG-4 & & 2800 & 1818 & & Cunniff and Chintawong (1985) \\
\hline DA60 & IWEINC1 & $1 / 25 / 91$ & NMSUPG-4 & & 2520 & & & Files SWTDI \\
\hline DA60 & PTOPG4 & $8 / 2 / 86$ & NMSU PG-4 & & & 1770 & & Files SWTDI \\
\hline ED1 & 81 & $7 / 27 / 61$ & Clayton Well & 31.0 & 3030 & & & WATSTORE 1993 \\
\hline ED1 & 82 & $7 / 27 / 61$ & Clayton Well & 31.0 & 3030 & & & WATSTORE 1993 \\
\hline GR1 & GS15106 & $12 / 5 / 74$ & Mimbres Hot Springs \#3 & 61.1 & 450 & & & Summers (1976) \\
\hline GR1 & MIML3-12-65 & $12 / 29 / 65$ & Mimbres Hot Springs \#3 & 62.3 & 430 & 221 & & Summers (1976) \\
\hline GR1 & MIML3-WR & $10 / 3 / 65$ & Mimbres Hot Springs \#3 & & 444 & & & Summers (1976) \\
\hline GR1 & MIML3-12-74 & $12 / 5 / 74$ & Mimbres Hot Springs \#3 & & & & & Summers (1976) \\
\hline GR1 & MIML3-8-65 & $8 / 28 / 65$ & Mimbres Hot Springs \#3 & & 422 & 285 & & Summers (1976) \\
\hline GR2 & $E \mid 3024$ & $10 / ? / 85$ & Gila Hot Springs Doyle Well & 74.0 & & & & Files SWTDI \\
\hline GR3 & SNM-16 & $6 / 3 / 87$ & Turkey Creek Hot Spring & 65.0 & 326 & 260.4 & & Shevenell (1987) \\
\hline GR3 & GILA22 & $? / ? / 75-80$ & Turkey Creek Hot Spring & 69.8 & & 260 & & Swanberg (1980) \\
\hline GR3 & SNM-17 & $6 / 3 / 87$ & Turkey Creek Hot Spring & 73.0 & 329 & 258.8 & & Shevenell (1987) \\
\hline
\end{tabular}




\begin{tabular}{|c|c|c|c|c|c|c|c|c|}
\hline SITE ID & SAMPLE & DATE & NAME & $\begin{array}{c}T M P \\
C \\
\end{array}$ & $\begin{array}{l}\text { COND } \\
\text { uS/cm }\end{array}$ & $\begin{array}{c}\text { TDS } \\
m g / L\end{array}$ & $\begin{array}{l}\text { FLOW } \\
\text { L/min }\end{array}$ & REFERENCE \\
\hline GR3 & GILA20 & $? / ? / 75-80$ & Turkey Creek Hot Spring & 74.0 & & 236 & & Swanberg (1980) \\
\hline GR4 & SNM-12 & $6 / 2 / 87$ & Gila Hot Springs Campbell \#4 Well & 71.1 & 659 & 495.8 & & Shevenell (1987) \\
\hline GR4 & El6968 & $10 / 10 / 86$ & Gila Hot Springs Campbell \#4 Well & 72.0 & & & & Files SWTDI \\
\hline GR5 & GHS0920 & $10 / 6 / 81$ & Gila Spring Hot Spring \#14 & 66.0 & 640 & & & Schwab and others (1982) \\
\hline GR5 & GHS0925 & $10 / 6 / 81$ & Gila Spring Hot Spring \#14 & 66.0 & & 445 & 3.16 & Schwab and others (1982) \\
\hline GR6 & NB82-32 & ?/?/80-82 & Gila HS northern artesian well & 65.0 & & & & Norman and Bernhardt (1982) \\
\hline GR7 & GHS-66 & $2 / 17 / 66$ & Gila Hot Springs & 41.5 & 560 & 496 & & Summers (1976) \\
\hline GR7 & NB82-31 & $? / ? / 80-82$ & Gila Hot Springs & 59.0 & & & & Norman and Bernhardt (1982) \\
\hline GR7 & 117 & $12 / 5 / 74$ & Gila Hot Springs & 61.0 & 620 & & & WATSTORE 1993 \\
\hline GR7 & GILA5 & $? / ? / 75-80$ & Gila Hot Springs & 62.8 & 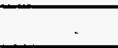 & 408 & & Swanberg (1980) \\
\hline GR7 & GHS-74 & $12 / 5 / 74$ & Gila Hot Springs & 63.9 & 620 & & & Summers (1976) \\
\hline GR7 & GS31051 & $12 / 5 / 74$ & Gila Hot Springs & 63.89 & & & & Summers (1976) \\
\hline GR7 & GS36105 & $6 / 23 / 57$ & Gila Hot Springs & 63.9 & 653 & 369 & 94.64 & Summers (1976) \\
\hline GR7 & GS4897 & $7 / 25 / 62$ & Gila Hot Springs & 63.9 & 638 & 421 & 378.54 & Summers (1976) \\
\hline GR7 & 115 & $6 / 23 / 57$ & Gila Hot Springs & 64.0 & 653 & 369 & 567.75 & WATSTORE 1993 \\
\hline GR7 & 116 & $7 / 25 / 62$ & Gila Hot Springs & 64 & 638 & 421 & & WATSTORE 1993 \\
\hline GR7 & SNM-13 & $6 / 2 / 87$ & Gila Hot Springs & 65.2 & 642 & 483.5 & & Shevenell (1987) \\
\hline GR7 & GILA6 & $? / ? / 75-80$ & Gila Hot Springs & 66.3 & & 416 & & Swanberg (1980) \\
\hline GR7 & M77-GR1 & $? / ? / 77$ & Gila Hot Springs & 68.0 & & 468 & & Mariner and others (1977) \\
\hline GR9 & NB82-30 & $? / ? / 80-82$ & Gila HS middle artesian well & 62.0 & & & & Norman and Bernhardt (1982) \\
\hline GR10 & GHS2252 & $10 / 7 / 81$ & Gila Hot Springs Campbell \#2 Well & 60.0 & & 428 & 3.28 & Schwab and others (1982) \\
\hline GR10 & GHS0700 & $10 / 8 / 81$ & Gila Hot Springs Campbell \#2 Well & 61.0 & & 423 & 3.27 & Schwab and others (1982) \\
\hline GR10 & GHS0711 & $10 / 8 / 81$ & Gila Hot Springs Campbell \#2 Well & 61.0 & & & & Schwab and others (1982) \\
\hline GR10 & GHS1445 & $10 / 7 / 81$ & Gila Hot Springs Campbell \#2 Well & 61.0 & 620 & 418 & 3.268 & Schwab and others (1982) \\
\hline GR10 & GHS14006 & $10 / 9 / 81$ & Gila Hot Springs Campbell \#2 Well & 62.0 & 550 & & 3.16 & Schwab and others (1982) \\
\hline GR10 & GHS1400F & $10 / 9 / 81$ & Gila Hot Springs Campbell \#2 Well & 62.0 & & 411 & & Schwab and others (1982) \\
\hline GR10 & GHS1610 & $10 / 7 / 81$ & Gila Hot Springs Campbell \#2 Well & 62.0 & 590 & 415 & 3.16 & Schwab and others (1982) \\
\hline GR11 & MIM-L25 & $12 / 29 / 65$ & Mimbres Hot Springs \#25 & 59.0 & & & & Summers (1976) \\
\hline GR12 & MIM-L32 & $12 / 29 / 65$ & Mimbres Hot Springs \#28 & 45.3 & & & & Summers (1976) \\
\hline GR12 & $M \mid M-L 28$ & $6 / 5 / 52$ & Mimbres Hot Springs \#28 & 58.1 & 452 & 308 & & Summers (1976) \\
\hline GR13 & GHS0929 & $10 / 6 / 81$ & Gila Spring Hot Spring \#41 & 58.0 & 560 & & & Schwab and others (1982) \\
\hline GR13 & GHS0935 & $10 / 6 / 81$ & Gila Spring Hot Spring \#41 & 58.0 & & 426 & 0.06 & Schwab and others (1982) \\
\hline GR14 & 85 & $6 / 5 / 52$ & Mimbres Hot Springs & 58.0 & 450 & & & WATSTORE 1993 \\
\hline GR14 & NB82-25 & $? / ? / 80-82$ & Mimbres Hot Springs & 58.0 & & & & Norman and Bernhardt (1982) \\
\hline GR14 & GILA4 & $? / ? / 75-80$ & Mimbres Hot Springs & 58.2 & & 320 & & Swanberg (1980) \\
\hline GR14 & 86 & $12 / 5 / 74$ & Mimbres Hot Springs & 60.5 & 455 & & & WATSTORE 1993 \\
\hline GR15 & GS19645 & $6 / 5 / 52$ & Mimbres Hot Springs \#8 & 57.5 & 450 & & & Summers (1976) \\
\hline GR16 & MIM-L12 & $12 / 29 / 65$ & Mimbres Hot Springs \#12 & 56.4 & & & & Summers (1976) \\
\hline GR17 & MIM-L21 & $12 / 29 / 65$ & Mimbres Hot Springs \#21 & 55.0 & & & & Summers (1976) \\
\hline
\end{tabular}




\begin{tabular}{|c|c|c|c|c|c|c|c|c|}
\hline SITE ID & SAMPLE & $\overline{D A T E}$ & NAME & $\begin{array}{c}T M P \\
C\end{array}$ & $\begin{array}{l}\text { COND } \\
u S / \mathrm{cm}\end{array}$ & $\begin{array}{c}\text { TDS } \\
m g / L\end{array}$ & $\begin{array}{c}\text { FLOW } \\
L \text { min }\end{array}$ & REFERENCE \\
\hline GR18 & MIM-L23 & $12 / 29 / 65$ & Mimbres Hot Springs \#23 & 54.4 & & & 9.84 & Summers (1976) \\
\hline GR19 & 75 & $2 / 5 / 76$ & Faywood Hot Springs & 52.0 & 560 & & & WATSTORE 1993 \\
\hline GR19 & 73 & $4 / 19 / 57$ & Faywood Hot Springs & 53 & 605 & 384 & 189.25 & WATSTORE 1993 \\
\hline GR19 & GS1856 & $4 / 19 / 57$ & Faywood Hot Springs & 53.3 & 605 & 384 & 189.27 & Summers (1976) \\
\hline GR19 & GS27917 & $11 / 9 / 54$ & Faywood Hot Springs & 53.3 & 600 & & 189.27 & Summers (1976) \\
\hline GR19 & 74 & $12 / 5 / 74$ & Faywood Hot Springs & 53.5 & 603 & & & WATSTORE 1993 \\
\hline GR19 & GILA2 & $? / ? / 75-80$ & Faywood Hot Springs & 53.8 & & 492 & & Swanberg (1980) \\
\hline GR19 & NB82-26 & $? / ? / 80-82$ & Faywood Hot Springs & 55.0 & & & & Norman and Bernhardt (1982) \\
\hline GR19 & FAY-S76-2 & $12 / 26 / 65$ & Faywood Hot Springs & & 560 & 384 & & Summers (1976) \\
\hline GR19 & FAY-WRD & $12 / 26 / 65$ & Faywood Hot Springs & & 504 & 330 & & Summers (1976) \\
\hline GR19 & GS19824 & $6 / 2 / 52$ & Faywood Hot Springs & & 606 & & & Summers (1976) \\
\hline GR19 & FAY-S76-1 & $12 / 5 / 74$ & Faywood Hot Springs & & & & & Summers (1976) \\
\hline GR19 & GS15112 & $12 / 5 / 74$ & Faywood Hot Springs & & 603 & & & Summers (1976) \\
\hline GR20 & SPS-LL66 & $2 / 20 / 66$ & Gila Lyons Lodge swimming pool HS & 51.7 & 390 & 335 & 18.93 & Summers (1976) \\
\hline GR20 & GS36104 & $6 / 23 / 57$ & Gila Lyons Lodge swimming pool HS & & 432 & & 37.85 & Summers (1976) \\
\hline GR21 & 114 & $6 / 23 / 57$ & well & 52.0 & 432 & & 37.85 & WATSTORE 1993 \\
\hline GR22 & M77-GR2 & $? / ? / 77$ & Gila River Waterfall HS & 43.0 & & 558 & & Mariner and others (1977) \\
\hline GR22 & GILA8 & $? / ? / 75-80$ & Gila River Waterfall HS & 43.6 & & 516 & & Swanberg (1980) \\
\hline GR22 & WFALLHS & $3 / 27 / 66$ & Gila River Waterfall HS & 44.7 & 680 & 518 & 75.7 & Summers (1976) \\
\hline GR23 & EFGR-NM & $2 / 22 / 66$ & Gila East Fork no name spring & 41.39 & 581 & 369 & 111.35 & Summers (1976) \\
\hline GR23 & EFGR-SUM & $2 / 22 / 66$ & Gila East Fork no name spring & 41.4 & 560 & 367 & 117.35 & Summers (1976) \\
\hline GR25 & M77-GR3 & $? / ? / 77$ & Gila East Fork Hot Spring & 36.0 & & 385 & & Mariner and others (1977) \\
\hline GR26 & EFS-LL66 & $2 / 20 / 66$ & Gila Lyons Lodge East Fork Hot Springs & 35.6 & 500 & 358 & & Summers (1976) \\
\hline GR26 & WSM-LL66 & $2 / 20 / 66$ & Gila Lyons Lodge HS midpoint & 45.6 & & & & Summers (1976) \\
\hline GR26 & WSC-LL66 & $2 / 20 / 66$ & Gila Lyons Lodge HS composite & & 580 & 407 & 75.7 & Summers (1976) \\
\hline GR26 & WSD-LL66 & $2 / 20 / 66$ & Gila Lyons Lodge HS downstream & & & & & Summers (1976) \\
\hline GR26 & WSU-LL66 & $2 / 20 / 66$ & Gila Lyons Lodge HS upstream & & & & & Summers (1976) \\
\hline GR27 & KWS-W3-1 & $965-196$ & Kennecott Warm Spring Well \#3 & 34.6 & 350 & 281 & & Summers (1976) \\
\hline GR27 & KWS-W3-2 & $5 / 17 / 72$ & Kennecott Warm Spring Well \#3 & & 482 & 320 & & Summers (1976) \\
\hline GR29 & NB82-27 & $? / ? / 80-82$ & Kennecott Warm Spring Well \#3 & 34.0 & & & & Norman and Bernhardt (1982) \\
\hline GR30 & GS50019 & $7 / 14 / 62$ & Cliff Warm Well & 33.3 & 665 & 435 & & Summers (1976) \\
\hline GR30 & 94 & $7 / 14 / 62$ & Cliff Warm Well & 33.5 & 665 & 435 & 37.85 & WATSTORE 1993 \\
\hline GR30 & S76CLIFF & $3 / 3 / 66$ & Cliff Warm Well & 35.0 & 610 & 439 & & Summers (1976) \\
\hline GR32 & LD2 & $? / ? / 75-80$ & Muir Ranch well & 33.0 & & 816 & & Swanberg (1980) \\
\hline GR34 & 87 & $9 / 22 / 54$ & well & 32.0 & 754 & & 1892.5 & WATSTORE 1993 \\
\hline GR35 & 84 & $5 / 13 / 65$ & well & 31.1 & 421 & 272 & & WATSTORE 1993 \\
\hline GR36 & GS29750 & $4 / 26 / 55$ & Spring Canyon Warm Spring & 28.9 & 472 & 311 & & Summers (1976) \\
\hline GR39 & GILA23 & $? / ? / 75-80$ & Riverside Well & 29.0 & & 292 & & Swanberg (1980) \\
\hline GR39 & GS29790 & $6 / 6 / 55$ & Riverside Well & 30.0 & 551 & 363 & & Summers (1976) \\
\hline
\end{tabular}




\begin{tabular}{|c|c|c|c|c|c|c|c|c|}
\hline SITE ID & SAMPLE & DATE & NAME & $\begin{array}{c}T M P \\
C\end{array}$ & $\begin{array}{l}\text { COND } \\
\text { uS/Cm }\end{array}$ & $\begin{array}{c}\text { TDS } \\
\mathrm{mg} / \mathrm{L}\end{array}$ & $\begin{array}{l}\text { FLOW } \\
\text { L/min }\end{array}$ & REFERENCE \\
\hline GR39 & GILA24 & $? / ? 775-80$ & Spring Canyon Warm Spring & 31.0 & & 332 & & Swanberg (1980) \\
\hline GR39 & NB82-28 & ?/?/80-82 & Riverside Well & 38.0 & & 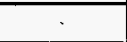 & & Norman and Bernhardt (1982) \\
\hline GR41 & GILA29 & $? / ? / 75-80$ & Mangas Springs & 27.0 & & 544 & & Swanberg (1980) \\
\hline GR42 & J1 & $? / ? / 75-80$ & Allen Spring & 25.6 & & 492 & & Swanberg (1980) \\
\hline GR43 & $\sqrt{7}$ & $? / ? / 75-80$ & Cliff Warm Spring & 25.0 & & 164 & & Swanberg (1980) \\
\hline GR43 & T72P193 & $9 / 14 / 55$ & Cliff Warm Spring & 25.0 & 256 & & & Summers (1976) \\
\hline GR44 & ATWS-W4-1 & $5 / 12 / 72$ & Apache Tejo Warms Springs Well \#4 & & 520 & 370 & & Summers (1976) \\
\hline GR44 & ATWS-W4-2 & $5 / 12 / 72$ & Apache Tejo Warms Springs Well \#4 & & 634 & 868 & & Summers (1976) \\
\hline GR45 & ATWS-W5 & $5 / 12 / 72$ & Apache Tejo Warms Springs Well \#5 & & & & & Summers (1976) \\
\hline HD1 & SNM-11 & $6 / 2 / 87$ & Lightning Dock Burgett Well \#1 & 104.0 & 1666 & 1180.5 & & Shevenell (1987) \\
\hline $\mathrm{HD} 2$ & 35 & $4 / 28 / 49$ & Lightning Dock hot well & 98.0 & 1540 & & & WATSTORE 1993 \\
\hline HD2 & GS59532 & $4 / 30 / 66$ & Lightning Dock hot well & & 1560 & & & Summers (1976) \\
\hline HD2 & S76LD-12 & $4 / 30 / 66$ & Lightning Dock hot well & & 1500 & 1057 & & Summers (1976) \\
\hline HD3 & NB82-40 & $? / ? / 80-82$ & Lightning Dock hot well & 96.0 & & & & Norman and Bernhardt (1982) \\
\hline HD4 & 36 & $4 / 30 / 66$ & Lightning Dock hot well & 95.5 & 1560 & & & WATSTORE 1993 \\
\hline HD4 & S76LD-10 & $4 / 27 / 54$ & Lightning Dock hot well & & 1580 & 1160 & & Summers (1976) \\
\hline HD5 & SNM-10 & $6 / 2 / 87$ & Lighting Dock Burgett Well \#10 & 95.2 & 1621 & 1136.6 & & Shevenell (1987) \\
\hline HD6 & 37 & $4 / 10 / 55$ & Lightning Dock hot well & 94.0 & 1510 & & 757 & WATSTORE 1993 \\
\hline HD7 & $\mathrm{P} 2$ & $? / ? / 75-80$ & Lightning Dock hot well & 85.0 & & 1116 & & Swanberg (1980) \\
\hline HD8 & P3 & $? / ? / 75-80$ & Lightning Dock McCants Well & 81.0 & & 1024 & & Swanberg (1980) \\
\hline HD9 & $\mathrm{P4}$ & $? / ? / 75-80$ & Lightning Dock hot well & 71.0 & & 1608 & & Swanberg (1980) \\
\hline HD10 & 33 & $4 / 30 / 66$ & Lightning Dock hot well & 52 & 2310 & & & WATSTORE 1993 \\
\hline HD10 & GS59533 & $4 / 30 / 66$ & Lightning Dock hot well & & 2310 & 1680 & & Summers (1976) \\
\hline HD10 & S76LD-5 & $3 / 2 / 66$ & Lightning Dock hot well & & 2260 & 1732 & & Summers (1976) \\
\hline HD10 & S76LD-2 & $12 / 12 / 58$ & Lightning Dock hot well & & & 1055 & & Summers (1976) \\
\hline HD10 & S76LD-3 & $4 / 5 / 60$ & Lightning Dock hot well & & 1577 & 1112 & & Summers (1976) \\
\hline HD10 & S76LD-4 & $8 / 2 / / 65$ & Lightning Dock hot well & & 2200 & 1761 & & Summers (1976) \\
\hline HD10 & S76LD-6 & $4 / 30 / 66$ & Lightning Dock hot well & & 2200 & 1786 & & Summers (1976) \\
\hline HD11 & S76P15-2 & $7 / 8 / 55$ & warm well & 31.1 & 1590 & & & Summers (1976) \\
\hline HD11 & S76P15-3 & $6 / 20 / 66$ & warm well & 31.4 & & & 37 & Summers (1976) \\
\hline HD11 & S76P15-1 & $6 / 14 / 55$ & warm well & 35.0 & & & & Summers (1976) \\
\hline HD12 & 34 & $4 / 30 / 66$ & Lightning Dock hot well & 33.5 & 1960 & & & WATSTORE 1993 \\
\hline HD12 & GS59534 & $4 / 30 / 66$ & Lightning Dock hot well & & 1960 & 1380 & & Summers (1976) \\
\hline HD12 & S76LD-1 & $4 / 30 / 66$ & Lightning Dock hot well & & 1760 & 1295 & & Summers (1976) \\
\hline HD13 & 1 & $8 / 22 / 56$ & well & 32.0 & 1020 & & & WATSTORE 1993 \\
\hline HD14 & 63 & $7 / 8 / 55$ & Blowing Well & 31.0 & 1590 & & 18.93 & WATSTORE 1993 \\
\hline HD15 & 68 & $8 / 17 / 81$ & well & 30.0 & 460 & & & WATSTORE 1993 \\
\hline HD17 & S76LD-11 & $4 / 10 / 55$ & Lightning Dock hot well & & 1510 & 1110 & & Summers (1976) \\
\hline HD18 & S76LD-13 & $4 / 30 / 66$ & Lightning Dock hot well & & & & & Summers (1976) \\
\hline
\end{tabular}




\begin{tabular}{|c|c|c|c|c|c|c|c|c|}
\hline SITE ID & SAMPLE & DATE & NAME & $\begin{array}{c}T M P \\
C\end{array}$ & $\begin{array}{l}\text { COND } \\
\text { uS/cm }\end{array}$ & $\begin{array}{c}\text { TDS } \\
\mathrm{mg} / \mathrm{L}\end{array}$ & $\begin{array}{c}\text { FLOW } \\
\text { L/min }\end{array}$ & REFERENCE \\
\hline HD19 & S76LD-14 & $4 / 4 / 60$ & Lightning Dock hot well & & 835 & 620 & & Summers (1976) \\
\hline HD20 & S76LD-7A & $2 / 1 / 49$ & Lightning Dock hot well & & 1650 & 1020 & & Summers (1976) \\
\hline $\mathrm{HD} 20$ & S76LD-7B & $4 / 28 / 49$ & Lightning Dock hot well & & 1540 & 1130 & & Summers (1976) \\
\hline $\mathrm{HD20}$ & S76LD-8 & $7 / 30 / 51$ & Lightning Dock hot well & & 1600 & & & Summers (1976) \\
\hline HD20 & S76LD-9 & $3 / 28 / 52$ & Lightning Dock hot well & & 1600 & & & Summers (1976) \\
\hline LA1 & 386 & $1 / 15 / 61$ & Pueblo Canyon \#7 Well & 30.5 & 729 & & 1816.8 & WATSTORE 1993 \\
\hline LA1 & 385 & $1 / 10 / 61$ & Pueblo Canyon \#7 Well & 31 & 766 & & 1816.8 & WATSTORE 1993 \\
\hline LU1 & S76-24SR7W & $8 / 4 / 69$ & Smyer Well & 38.9 & 439 & & & Summers (1976) \\
\hline LU1 & 42 & $8 / 4 / 69$ & Smyer Well & 39.0 & 439 & & & WATSTORE 1993 \\
\hline LU3 & 41 & $2 / 1 / 45$ & well & 34.5 & 515 & & & WATSTORE 1993 \\
\hline LU4 & 6 & $8 / 8 / 52$ & well & 33.0 & 1620 & & 1400.5 & WATSTORE 1993 \\
\hline LU6 & 40 & $1 / 5 / 54$ & well & 31.5 & 824 & & & WATSTORE 1993 \\
\hline LU7 & PAL4 & $? / ? / 75-80$ & well & 31.5 & & 700 & & Swanberg (1980) \\
\hline LU8 & W86 & $? / ? / 75-80$ & well & 31.1 & & 732 & & Swanberg (1980) \\
\hline LU9 & 8 & $5 / 22 / 58$ & well & 30.5 & 1860 & 1130 & & WATSTORE 1993 \\
\hline LU9 & 7 & $8 / 8 / 52$ & well & 31.0 & 1850 & & 832.7 & WATSTORE 1993 \\
\hline LU13 & 50 & $9 / 2 / 82$ & Little Ed Well & 30.0 & 500 & & 3.79 & WATSTORE 1993 \\
\hline LU14 & W69 & $? / ? / 75-80$ & well & 30.0 & & 1168 & & Swanberg (1980) \\
\hline LU15 & 5 & $8 / 26 / 82$ & well & 30.0 & 1100 & & 378.5 & WATSTORE 1993 \\
\hline MK1 & 234 & $8 / 4 / 50$ & Fort Wingate Well/Santa Fe 'Spring' & 55.0 & 730 & & 87.06 & WATSTORE 1993 \\
\hline $\mathrm{MK1}$ & GS64891 & $10 / 2 / 68$ & Fort Wingate Well/Santa Fe 'Spring' & 61.1 & 5520 & & & Summers (1976) \\
\hline MK2 & 229 & $11 / 21 / 56$ & well & 46.0 & 3110 & & 809.99 & WATSTORE 1993 \\
\hline MK3 & $\mathrm{DL}-4-N M$ & $4 / 2 / 79$ & Ya-Ta-Hey Well & 45.5 & & & & Levitte and Gambill (1980) \\
\hline MK4 & 432 & $10 / 22 / 87$ & well & 37.4 & 1600 & & & WATSTORE 1993 \\
\hline MK4 & 430 & $4 / 24 / 86$ & well & 42.2 & 1800 & & & WATSTORE 1993 \\
\hline MK4 & 431 & $4 / 24 / 86$ & well & 42.2 & 1800 & & & WATSTORE 1993 \\
\hline MK5 & 231 & $3 / 5 / 86$ & well & 40.2 & 2850 & 2330 & & WATSTORE 1993 \\
\hline MK6 & 242 & $1 / 31 / 68$ & well & 39.0 & 890 & 565 & & WATSTORE 1993 \\
\hline MK7 & 299 & $4 / 1 / 60$ & Navajo well & 36 & 594 & & 3785 & WATSTORE 1993 \\
\hline MK7 & 298 & $4 / 28 / 54$ & Navajo well & 37.5 & 703 & & 3785 & WATSTORE 1993 \\
\hline MK8 & DL-15-NM & $6 / 2 / 79$ & Toh Sah Toh & 37.0 & & & 1135 & Levitte and Gambill (1980) \\
\hline MK9 & 240 & $1 / 24 / 69$ & well & 34 & 1360 & & & WATSTORE 1993 \\
\hline MK9 & 239 & $1 / 12 / 69$ & well & 36 & 1570 & & & WATSTORE 1993 \\
\hline MK10 & 264 & $7 / 14 / 81$ & NTUA \#2 Well & 36.0 & 500 & & & WATSTORE 1993 \\
\hline MK11 & 261 & $7 / 14 / 81$ & well & 35.5 & 450 & & & WATSTORE 1993 \\
\hline MK12 & 296 & $3 / 12 / 75$ & Pure Oil Navajo \#1 (Tohachi Well) & 35 & 644 & & & WATSTORE 1993 \\
\hline MK12 & GS52896 & $9 / 9 / 63$ & Pure Oil Navajo \#1 (Tohachi Well) & 35.6 & 606 & & 3406.9 & Summers (1976) \\
\hline MK12 & GS44727 & $4 / 1 / 60$ & Pure Oil Navajo \#1 Well (Tohachi Well) & 36.1 & 594 & 390 & 3406.9 & Summers (1976) \\
\hline MK12 & 297 & $8 / 28 / 85$ & Pure Oil Navajo \#1 (Tohachi Well) & 37.0 & 655 & & 1922.8 & WATSTORE 1993 \\
\hline
\end{tabular}




\begin{tabular}{|c|c|c|c|c|c|c|c|c|}
\hline SITE ID & SAMPLE & DATE & NAME & $\begin{array}{c}T M P \\
C \\
\end{array}$ & $\begin{array}{l}\text { COND } \\
\text { uS/cm }\end{array}$ & $\begin{array}{c}\text { TDS } \\
\mathrm{mg} / \mathrm{L}\end{array}$ & $\begin{array}{l}\text { FLOW } \\
\text { L/min }\end{array}$ & REFERENCE \\
\hline MK12 & NM51 & $? / ? / 75-80$ & Pure Oil Navajo \#1 Well (Tohachi Well) & & & 376 & & Swanberg (1980) \\
\hline MK13 & S76NAVAJO & $3 / 13 / 75$ & Pure Oil Navajo \#3 Well & 35.0 & & & & Summers (1976) \\
\hline MK14 & 265 & $8 / 17 / 82$ & Mobil Well Crown Point & 33.5 & 415 & & & WATSTORE 1993 \\
\hline MK15 & 420 & $5 / 10 / 67$ & well & 33.5 & 951 & & & WATSTORE 1993 \\
\hline MK16 & 262 & $7 / 15 / 81$ & well & 33.0 & 450 & & & WATSTORE 1993 \\
\hline MK17 & 243 & $6 / 26 / 75$ & well & 32.5 & 1500 & 906 & 2267.2 & WATSTORE 1993 \\
\hline MK18 & 423 & $4 / 21 / 86$ & well & 32.5 & 2900 & & & WATSTORE 1993 \\
\hline MK18 & 422 & $4 / 21 / 86$ & well & 32.7 & 2900 & & & WATSTORE 1993 \\
\hline MK18 & 421 & $5 / 10 / 67$ & well & 35.5 & 2910 & & & WATSTORE 1993 \\
\hline MK19 & 235 & $12 / 22 / 33$ & well & 32.0 & & & & WATSTORE 1993 \\
\hline MK20 & 241 & $7 / 31 / 70$ & well & 32 & 1280 & 860 & 2649.5 & WATSTORE 1993 \\
\hline MK21 & 237 & $12 / 6 / 55$ & well & 31.5 & 1220 & 802 & 946.25 & WATSTORE 1993 \\
\hline MK22 & 202 & $5 / 6 / 64$ & well & 30.5 & 1140 & 730 & & WATSTORE 1993 \\
\hline MK23 & 256 & $10 / 2 / 87$ & well & 30.5 & 800 & & & WATSTORE 1993 \\
\hline MK24 & 260 & $7 / 14 / 81$ & well & 30.0 & 675 & & & WATSTORE 1993 \\
\hline OT1 & $45 \mathrm{N9}$ & $? / ? / 45$ & N-9 Well & 71.1 & & 12500 & & Henry and Gluck (1981) \\
\hline OT2 & $57 \mathrm{~N} 11$ & $1 / 2 / 57$ & $\mathrm{~N}-11$ Well & 61.1 & 14500 & 8980 & & Henry and Gluck (1981) \\
\hline OT3 & 26 & $10 / 18 / 56$ & $M-11$ & 33.5 & 1980 & 1040 & & WATSTORE 1993 \\
\hline OT3 & $56 \mathrm{M11A}$ & $10 / ? / 56$ & $M-11($ DST $465-504 \mathrm{ft})$ Well & 50.0 & 2050 & 1120 & & Henry and Gluck (1981) \\
\hline OT3 & $56 \mathrm{M} 11 \mathrm{~B}$ & $12 / ? / 56$ & M-11 Well & hot & 2030 & 1170 & & Henry and Gluck (1981) \\
\hline OT4 & 65 & $8 / 2 / 87$ & FLOUR-1 Well & 30.0 & 18400 & & 1199.9 & WATSTORE 1993 \\
\hline OT4 & 64 & $7 / 15 / 87$ & FLOUR-1 Well & 33.5 & 18000 & & & WATSTORE 1993 \\
\hline OT5 & S76GART4 & $12 / 17 / 65$ & Garton Well & 33.7 & 10000 & 9086 & & Summers (1976) \\
\hline OT5 & SA1 & $? / ? / 75-80$ & Garton Well & 34.0 & & 9140 & & Swanberg (1980) \\
\hline OT5 & S76GART1 & $? / 2 / 29$ & Garton Well & & & 7580 & 4164 & Summers (1976) \\
\hline OT5 & S76GART2 & $3 / 29 / 35$ & Garton Well & & & 9111 & 75 & Summers (1976) \\
\hline OT5 & S76GART3 & $8 / 12 / 64$ & Garton Well & & 16000 & 10240 & 30 & Summers (1976) \\
\hline OT6 & OLDN8 & $1900(?)$ & N-8 Well & hot (?) & & & & Henry and Gluck (1981) \\
\hline OT7 & 27 & $1 / 28 / 57$ & $\mathrm{~N}-11$ & 61.0 & 14500 & 9010 & 132.48 & WATSTORE 1993 \\
\hline RA1 & $\mathrm{OC}-17$ & $4 / ? / 82$ & Ojo Caliente Hot Spring Soda Spring & 27.3 & 4220 & 3673 & & Vuataz and others (1984) \\
\hline RA1 & GS9877 & $10 / 1 / 74$ & Ojo Caliente Hot Spring Soda Spring & 35.0 & 3890 & & & Summers (1976) \\
\hline RA1 & GS13378 & $10 / 6 / 49$ & Ojo Caliente Hot Spring Soda Spring & & 3910 & & & Summers (1976) \\
\hline RA1 & HILLEBRD & $? / ? / ?$ & Ojo Caliente Hot Spring Soda Spring & & & & & Summers (1976) \\
\hline RA2 & OC-18 & $5 / ? / 82$ & Ojo Caliente Hot Spring Well & 53.6 & 4560 & 3724 & & Vuataz and others (1984) \\
\hline RA2 & $\mathrm{OC}-4$ & $12 / ? / 79$ & Ojo Caliente Hot Spring Well & 54.0 & 3900 & 3344 & & Vuataz and others (1984) \\
\hline RA2 & OC-25 & $4 / 2 / 82$ & Ojo Caliente Hot Spring Well & 54.2 & 4440 & 3618 & & Vuataz and others (1984) \\
\hline RA2 & NM21 & $? / ? / 75-80$ & Ojo Callente Hot Spring Well & 55.6 & & 2576 & & Swanberg (1980) \\
\hline RA3 & $\mathrm{OC}-14$ & $4 / ? / 82$ & Ojo Caliente Arsenic Hot Spring & 38.3 & 4200 & 3665 & & Vuataz and others (1984) \\
\hline RA3 & S76ARSEN & $12 / 3 / 74$ & Ojo Caliente Arsenic Hot Spring & 38.3 & & & & Summers (1976) \\
\hline
\end{tabular}




\begin{tabular}{|c|c|c|c|c|c|c|c|c|}
\hline SITE ID & SAMPLE & DATE & NAME & $\begin{array}{c}\text { TMP } \\
C\end{array}$ & $\begin{array}{l}\text { COND } \\
\text { uS/cm }\end{array}$ & $\begin{array}{l}\text { TDS } \\
m g / L\end{array}$ & $\begin{array}{l}\text { FLOW } \\
\text { L/min }\end{array}$ & REFERENCE \\
\hline RA3 & NM19 & $? / ? / 75-80$ & Ojo Caliente Arsenic Hot Spring & 43.5 & & 2652 & & Swanberg (1980) \\
\hline RA3 & OC-5 & $7 / ? / 80$ & Ojo Caliente Arsenic Hot Spring & 43.5 & 4000 & 3131 & 20 & Vuataz and others (1984) \\
\hline RA3 & GS13193 & $10 / 6 / 49$ & Ojo Caliente Arsenic Hot Spring & 45.0 & 3930 & & & Summers (1976) \\
\hline RA4 & NB82-4 & $? / ? / 80-82$ & Ojo Caliente Hot Spring & 43.0 & & & & Norman and Bernhardt (1982) \\
\hline RA5 & OC-2 & $12 / ? / 79$ & Ojo Caliente HS Lithia Spring & 38.0 & 3900 & 3475 & & Vuataz and others (1984) \\
\hline RA5 & $\mathrm{OC}-13$ & $4 / 2 / 82$ & Ojo Caliente HS Lithia Spring & 41.9 & 4190 & 3630 & & Vuataz and others (1984) \\
\hline RA6 & OC-3 & $12 / 2 / 79$ & Ojo Caliente HS Sodium Sulfate Spring & 40.0 & 4100 & 3533 & 15 & Vuataz and others (1984) \\
\hline RA6 & GS13192 & $10 / 6 / 49$ & Ojo Caliente HS Sodium Sulfate Spring & 40.6 & 3920 & & & Summers (1976) \\
\hline RA6 & NM20 & $? / ? / 75-80$ & Ojo Caliente HS Sodium Sulfate spring & 41.1 & & 2668 & & Swanberg (1980) \\
\hline RA6 & $\mathrm{OC}-16$ & $4 / 2 / 82$ & Ojo Caliente HS Sodium Sulfate Spring & 41.3 & 4250 & 3628 & 45 & Vuataz and others (1984) \\
\hline RA6 & GS8978 & $10 / 1 / 47$ & Ojo Caliente HS Sodium Sulfate Spring & & 3890 & & & Summers (1976) \\
\hline RA7 & 450 & $12 / 3 / 74$ & Ojo Caliente Hot Spring Iron Spring & 40.0 & 390 & & & WATSTORE 1993 \\
\hline RA7 & OC-15 & $4 / ? / 82$ & Ojo Caliente Hot Spring Iron Spring & 42.2 & 4250 & 3674 & & Vuataz and others (1984) \\
\hline RA7 & GS15115 & $12 / 3 / 74$ & Ojo Caliente Hot Spring Iron Spring & 42.8 & 3900 & & & Summers (1976) \\
\hline RA7 & S76IRON2 & $12 / 3 / 74$ & Ojo Caliente Hot Spring Iron Spring & 42.8 & & & & Summers (1976) \\
\hline RA7 & OC-1 & $12 / ? / 79$ & Ojo Caliente Hot Spring Iron Spring & 43.0 & 4100 & 3578 & 40 & Vuataz and others (1984) \\
\hline RA7 & S76IRON1 & $11 / 30 / 65$ & Ojo Caliente Hot Spring Iron Spring & & 3300 & 2438 & & Summers (1976) \\
\hline RA8 & NB82-3 & $? / ? / 80-82$ & Statue Spring & 29.0 & & & & Norman and Bernhardt (1982) \\
\hline RA8 & OC-26 & $6 / 2 / 82$ & Statue Spring & 29.2 & 1660 & 1386 & 110 & Vuataz and others (1984) \\
\hline RA8 & 452 & $9 / 5 / 52$ & Statue Spring & 36.0 & 1740 & & & WATSTORE 1993 \\
\hline RA9 & OC-20 & $4 / 2 / 82$ & Ojo Caliente Hot Spring Well \#1 & 34.3 & 3000 & 2586 & & Vuataz and others (1984) \\
\hline RA9 & PH65-524 & $4 / 8 / 65$ & Ojo Caliente Hot Spring Well \#1 & & 2375 & 1515 & & Summers (1976) \\
\hline RA11 & NM18 & $? / ? / 75-80$ & spring & 25.5 & & 1072 & & Swanberg (1980) \\
\hline RA12 & PH65-523 & $4 / 8 / 65$ & Ojo Caliente Hot Spring Well \#2 & & 1050 & 665 & & Summers (1976) \\
\hline SA1 & BA-7 & $7 / 7 / 82$ & Jemez/Baca \# 15 & 267.0 & 10400 & 5735 & & Shevenell and others (1987) \\
\hline SA1 & $\mathrm{BA}-8$ & $9 / ? / 82$ & Jemez/Baca \# 15 & 326.0 & 10600 & 5783 & & Shevenell and others (1987) \\
\hline SA2 & BA-5 & $7 / ? / 82$ & Jemez/Baca \# 4 & 297.0 & 9100 & 4719 & & Shevenell and others (1987) \\
\hline SA3 & $\mathrm{BA}-2$ & $6 / ? / 82$ & Jemez/Baca \# 4 & 294.0 & 9100 & 4769 & & Shevenell and others (1987) \\
\hline SA4 & $B A-4$ & $7 / ? / 82$ & Jemez/Baca \# 13 & 279.0 & 8900 & 4644 & & Shevenell and others (1987) \\
\hline SA5 & $\mathrm{BA}-1$ & $6 / ? / 82$ & Jemez/Baca \#13 & 278.0 & 8500 & 4529 & & Shevenell and others (1987) \\
\hline SA6 & BA-3 & $6 / ? / 82$ & Jemez/Baca \#24 & 260.0 & 10600 & 5328 & & Shevenell and others (1987) \\
\hline SA6 & $\mathrm{BA}-6$ & $7 / ? / 82$ & Jemez/Baca \#24 & 261.0 & 10400 & 5339 & & Shevenell and others (1987) \\
\hline SA7 & VA-116 & $1 / ? / 83$ & Jemez/GRI WC 23-4 at $6300 \mathrm{ft}$ depth & 232.6 & 30800 & 18100 & & Shevenell and others (1987) \\
\hline SA8 & $B A-9$ & $10 / ? / 82$ & Jemez/Baca \# 19 & 223.0 & 10900 & 6147 & & Shevenell and others (1987) \\
\hline SA9 & VA-113 & $1 / ? / 83$ & Jemez/GRI WC 23-4 at $4800 \mathrm{ft}$ depth & 214.0 & 13720 & 8580 & & Shevenell and others (1987) \\
\hline SA9 & VA-114 & $1 / 2 / 83$ & Jemez/GRI WC 23-4 at $4800 \mathrm{ft}$ depth & 214.0 & & & & Shevenell and others (1987) \\
\hline SA10 & $S-6-80$ & $3 / ? / 82$ & Jemez/Sulphur Springs Women's Bathhouse & 88.0 & 12800 & 6850 & & Shevenell and others (1987) \\
\hline SA10 & VA-76 & $1 / ? / 82$ & Jemez/Sulphur Springs Women's Bathhouse & 89.0 & & & & Shevenell and others (1987) \\
\hline SA11 & VA-80 & $3 / ? / 80$ & Jemez/Sulphur Springs main fumarole & 88.0 & 70 & 95.3 & & Shevenell and others (1987) \\
\hline
\end{tabular}




\begin{tabular}{|c|c|c|c|c|c|c|c|c|}
\hline SITE ID & SAMPLE & DATE & NAME & $\begin{array}{c}\text { TMP } \\
C .\end{array}$ & $\begin{array}{l}\text { COND } \\
u S / \mathrm{cm}\end{array}$ & $\begin{array}{c}\text { TDS } \\
m g / L\end{array}$ & $\begin{array}{l}\text { FLOW } \\
\text { L/min }\end{array}$ & REFERENCE \\
\hline SA12 & VA-15 & $1 / 7 / 79$ & Jemez HS Jemez Springs \#1 Well $152 \mathrm{~m}$ & 60.5 & 1700 & 1140 & 80 & Shevenell and others (1987) \\
\hline SA12 & VA-21 & $2 / ? / 79$ & Jemez HS Jemez Springs \#1 Well 152 m & 61.0 & 1830 & 1200 & 20 & Shevenell and others (1987) \\
\hline SA12 & VA-19 & $1 / 7 / 79$ & Jemez HS Jemez Springs \#1 Well 24 m & 68.0 & 3300 & 2220 & 120 & Shevenell and others (1987) \\
\hline SA12 & VA-144 & $2 / ? / 84$ & Jemez HS Jemez Springs \#1 Well & 72.2 & 4670 & 3005 & & Shevenell and others (1987) \\
\hline SA12 & VA-121 & $1 / ? / 83$ & Jemez HS Jemez Springs \#1 Well & 73.3 & 4280 & 2530 & 64 & Shevenell and others (1987) \\
\hline SA12 & VA-25 & $5 / ? / 79$ & Jemez HS Jemez Springs \#1 Well 24 m & 73.3 & 3500 & 2560 & 8 & Shevenell and others (1987) \\
\hline SA13 & VA-7 & $1 / ? / 79$ & Jemez HS Travertine Mound Spring & 70.0 & 4200 & 7580 & 4 & Shevenell and others (1987) \\
\hline SA13 & VA-17 & $1 / ? / 79$ & Jemez HS Travertine Mound Spring & 72.0 & 4100 & 2600 & 4 & Shevenell and others (1987) \\
\hline SA13 & VA-66 & $12 / ? / 80$ & Jemez HS Travertine Mound Spring & 72.0 & 3400 & 1700 & 4 & Shevenell and others (1987) \\
\hline SA13 & VA-71 & $6 / 2 / 81$ & Jemez HS Travertine Mound Spring & 72.0 & 3900 & 2520 & 4 & Shevenell and others (1987) \\
\hline SA13 & VA-91 & $3 / ? / 82$ & Jemez HS Travertine Mound Spring & 72.3 & 4540 & & 4 & Shevenell and others (1987) \\
\hline SA13 & VA-123 & $1 / ? / 83$ & Jemez HS Travertine Mound Spring & 72.6 & 4360 & 2550 & 3 & Shevenell and others (1987) \\
\hline SA13 & VA-142 & $2 / ? / 84$ & Jemez HS Travertine Mound Spring & 72.9 & 4740 & 2630 & & Shevenell and others (1987) \\
\hline SA14 & 272 & $8 / 31 / 49$ & Jemez HS Soda Spring & 65.5 & 3560 & 2150 & 37.85 & WATSTORE 1993 \\
\hline SA15 & VA14 & $1 / 2 / 79$ & Jemez/Sulphur Springs unnamed HS & 63.0 & 5800 & 2490 & 2 & Shevenell and others (1987) \\
\hline SA16 & 268 & $2 / 7 / 74$ & Jemez Hot Springs & 31.5 & & & & WATSTORE 1993 \\
\hline SA16 & 267 & $5 / 18 / 73$ & Jemez Hot Springs & 49 & 3550 & & & WATSTORE 1993 \\
\hline SA16 & 266 & $8 / 21 / 24$ & Jemez Hot Springs & 52.0 & & 2184 & & WATSTORE 1993 \\
\hline SA16 & JEMEZ6 & $? / ? / 75-80$ & Jemez Hot Springs & 53.0 & & 1884 & & Swanberg (1980) \\
\hline SA16 & NB82-8 & $? / ? / 80-82$ & Jemez Hot Springs & 53.0 & & & & Norman and Bernhardt (1982) \\
\hline SA16 & JEMEZ5 & $? / ? / 75-80$ & Jemez Hot Springs & 56.0 & & 1952 & & Swanberg (1980) \\
\hline SA16 & 269 & $5 / 30 / 74$ & Jemez Hot Springs & 58 & 3460 & & & WATSTORE 1993 \\
\hline SA16 & 270 & $7 / 15 / 75$ & Jemez Hot Springs & 72.0 & 3250 & & & WATSTORE 1993 \\
\hline SA16 & 274 & $8 / 1 / 47$ & Jemez Hot Springs & 73.0 & 3700 & & & WATSTORE 1993 \\
\hline SA16 & JEMEZ7 & $? / ? / 75-80$ & Jemez Hot Springs & 74.0 & & 2156 & & Swanberg (1980) \\
\hline SA17 & 393 & $8 / 13 / 47$ & Jemez/Sulphur Springs Lemonade Spring & 53.0 & 3760 & & & WATSTORE 1993 \\
\hline SA17 & S-10-80 & $9 / 2 / 80$ & Jemez/Sulphur Springs Lemonade Spring & 58.0 & & 3220 & 0.5 & Shevenell and others (1987) \\
\hline SA18 & JP91-2 & $11 / 11 / 91$ & Jemez Pueblo \# 1 Well & 57.8 & 5560 & 3947 & & Goff (1991) \\
\hline SA18 & 91TD|1 & $1 / 5 / 91$ & Jemez Pueblo \#1 Well & 57.8 & & 3366 & 567.81 & Witcher (1991) \\
\hline SA18 & 91TD|3 & $6 / 15 / 91$ & Jemez Pueblo \#1 Well & 58.2 & & 3349 & 567.81 & Witcher and others (1992) \\
\hline SA19 & VA-18 & $1 / ? / 79$ & Jemez HS Gazebo Spring & 36.0 & 4250 & 2700 & & Shevenell and others (1987) \\
\hline SA19 & VA-93 & $3 / ? / 82$ & Jemez HS Gazebo Spring & 46.3 & 4270 & & 20 & Shevenell and others (1987) \\
\hline SA19 & VA-10 & $1 / 2 / 79$ & Jemez HS Gazebo Spring & 55.0 & 4200 & 2660 & 20 & Shevenell and others (1987) \\
\hline SA19 & VA-143 & $2 / 2 / 84$ & Jemez HS Gazebo Spring & 74.7 & 4460 & 2640 & & Shevenell and others (1987) \\
\hline SA19 & VA-122 & $1 / ? / 83$ & Jemez HS Gazebo Spring & 74.9 & 4380 & 2540 & 3 & Shevenell and others (1987) \\
\hline SA19 & VA-147 & $4 / 2 / 84$ & Jemez HS Gazebo Spring & & 3900 & & & Shevenell and others (1987) \\
\hline SA20 & 245 & $9 / 29 / 26$ & Kaseman \#2 Well (Zia Hot Well) & 32.0 & & 11200 & & WATSTORE 1993 \\
\hline SA20 & 249 & $4 / 2 / 45$ & Kaseman \#2 Well (Zia Hot Well) & 50.0 & 15400 & & & WATSTORE 1993 \\
\hline SA20 & 254 & $1 / 25 / 74$ & Kaseman \#2 Well (Zla Hot Well) & 51.0 & & & & WATSTORE 1993 \\
\hline
\end{tabular}




\begin{tabular}{|c|c|c|c|c|c|c|c|c|}
\hline SITE ID & SAMPLE & DATE & NAME & $\begin{array}{c}T M P \\
C \\
\end{array}$ & $\begin{array}{l}\text { COND } \\
u S / \mathrm{cm}\end{array}$ & $\begin{array}{c}\text { TDS } \\
m g / L\end{array}$ & $\begin{array}{c}\text { FLOW } \\
\text { L/min }\end{array}$ & REFERENCE \\
\hline SA20 & 252 & $6 / 5 / 73$ & Kaseman \#2 Well (Zia Hot Well) & 52 & 15700 & & 321.73 & WATSTORE 1993 \\
\hline SA20 & 253 & $6 / 6 / 73$ & Kaseman \#2 Well (Zia Hot Well) & 52.0 & & & & WATSTORE 1993 \\
\hline SA20 & $E$ & $2 / ? / 75-80$ & Kaseman \#2 Well (Zia Hot Well) & 52.0 & & 11300 & & Swanberg (1980) \\
\hline SA20 & 88TDI1 & $8 / ? / 88$ & Kaseman \#2 Well (Zia Hot Well) & 53.0 & & 10720 & & Witcher (1988a) \\
\hline SA20 & VA-125 & $2 / 2 / 83$ & Kaseman \#2 Well (Zia Hot Well) & 53.0 & 15700 & 11400 & 320 & Shevenell and others (1987) \\
\hline SA20 & VA-67 & $3 / ? / 81$ & Kaseman \#2 Well (Zia Hot Well) & 53.0 & 15800 & 11300 & 150 & Shevenell and others (1987) \\
\hline SA20 & VA-74 & $10 / ? / 81$ & Kaseman \#2 Well (Zia Hot Well) & 53.0 & 15600 & 12800 & 240 & Shevenell and others (1987) \\
\hline SA20 & NB82-10 & $? / ? / 80-82$ & Kaseman \#2 Well (Zia Hot Well) & 54.0 & & & & Norman and Bernhardt (1982) \\
\hline SA20 & VA-53 & $4 / ? / 80$ & Kaseman $\# 2$ Well (Zia Hot Well) & 54.0 & 16000 & 11700 & 150 & Shevenell and others (1987) \\
\hline SA20 & 247 & $3 / 14 / 64$ & Kaseman \#2 Well (Zia Hot Well) & 54.4 & 15300 & & 5677.5 & WATSTORE 1993 \\
\hline SA20 & VA-34 & $8 / ? / 79$ & Kaseman \#2 Well (Zia Hot Well) & 56.0 & 16600 & 12200 & 150 & Shevenell and others (1987) \\
\hline SA20 & 250 & $9 / 29 / 48$ & Kaseman \#2 Well (Zia Hot Well) & 59.0 & 15400 & & 1695.7 & WATSTORE 1993 \\
\hline SA20 & VA-149 & $4 / ? / 84$ & Kaseman \#2 Well (Zia Hot Well) & & 15500 & & & Shevenell and others (1987) \\
\hline SA21 & VA-92 & $3 / ? / 82$ & Jemez HS Buddhist Spring & 43.2 & 3200 & & 15 & Shevenell and others (1987) \\
\hline SA21 & VA-B & $1 / ? / 79$ & Jemez HS Buddhist Spring & 49.0 & 3300 & 2160 & 4 & Shevenell and others (1987) \\
\hline SA21 & VA-16 & $1 / ? / 79$ & Jemez HS Buddhist Spring & 50.0 & 3300 & 2550 & 4 & Shevenell and others (1987) \\
\hline SA22 & VA-12 & $1 / ? / 79$ & Jemez HS Marsh Spring & 49.0 & 4100 & 2620 & 4 & Shevenell and others (1987) \\
\hline SA23 & NB82-7 & $? / 2 / 80-82$ & Jemez/Soda Dam Hot Spring & 45.0 & & & & Norman and Bernhardt (1982) \\
\hline SA23 & 279 & $3 / 8 / 73$ & Jemez/Soda Dam Hot Spring & 46.0 & & & & WATSTORE 1993 \\
\hline SA23 & 280 & $6 / 29 / 73$ & Jemez/Soda Dam Hot Spring & 46.0 & 6500 & & & WATSTORE 1993 \\
\hline SA23 & VA-109 & $1 / ? / 83$ & Jemez/Soda Dam Hot Spring & 46.8 & 7090 & 4570 & 60 & Shevenell and others (1987) \\
\hline SA23 & VA-140 & $2 / 2 / 84$ & Jemez/Soda Dam Hot Spring & 46.8 & 7300 & 4570 & & Shevenell and others (1987) \\
\hline SA23 & JEMEZ2 & $? / ? / 75-80$ & Jemez/Soda Dam Hot Spring & 47.0 & & 3496 & & Swanberg $(1980)$ \\
\hline SA23 & $V A-132$ & $5 / ? / 83$ & Jemez/Soda Dam Hot Spring & 47.0 & 6800 & 4590 & & Shevenell and others (1987) \\
\hline SA23 & VA-26 & $5 / ? / 79$ & Jemez/Soda Dam Hot Spring & 47.0 & 6600 & 4630 & 60 & Shevenell and others (1987) \\
\hline SA23 & VA-51 & $4 / ? / 80$ & Jemez/Soda Dam Hot Spring & 47.0 & 5900 & 4150 & 60 & Shevenell and others (1987) \\
\hline SA23 & VA-6 & $7 / ? / 78$ & Jemez/Soda Dam Hot Spring & 47.0 & & 4014 & 60 & Shevenell and others (1987) \\
\hline SA23 & VA-64 & $12 / 2 / 80$ & Jemez/Soda Dam Hot Spring & 47.0 & 5600 & 4200 & 60 & Shevenell and others (1987) \\
\hline SA23 & VA-70 & $6 / 2 / 81$ & Jemez/Soda Dam Hot Spring & 47.0 & 6700 & 4380 & 60 & Shevenell and others (1987) \\
\hline SA23 & VA-73 & $10 / ? / 81$ & Jemez/Soda Dam Hot Spring & 47.0 & 6700 & 4539 & 40 & Shevenell and others (1987) \\
\hline SA23 & VA-89 & $3 / ? / 82$ & Jemez/Soda Dam Hot Spring & 47.0 & 6700 & & 40 & Shevenell and others (1987) \\
\hline SA23 & VA-99 & $8 / ? / 82$ & Jemez/Soda Dam Hot Spring & 47.5 & 6900 & & & Shevenell and others (1987) \\
\hline SA23 & VA-9 & $1 / ? / 79$ & Jemez/Soda Dam Hot Spring & 48.0 & 7050 & 4620 & 60 & Shevenell and others (1987) \\
\hline SA23 & 358 & $5 / 14 / 74$ & Jemez/Soda Dam Hot Spring & 76.0 & 13000 & & & WATSTORE 1993 \\
\hline SA23 & VA-146 & $4 / 2 / 84$ & Jemeż/Soda Dam Hot Spring & & 6700 & & & Shevenell and others (1987) \\
\hline SA24 & 283 & $7 / 15 / 75$ & Jemez'Soda Dam Hot Springs (west spring) & 46.0 & 5780 & & & WATSTORE 1993 \\
\hline SA24 & 284 & $8 / 7 / 75$ & Jemez'Soda Dam Hot Springs (west spring) & 50.0 & 5760 & & & WATSTORE 1993 \\
\hline SA25 & 292 & $11 / 7 / 72$ & Jemez/Spence Hot Spring & 39.5 & 276 & & 147.62 & WATSTORE 1993 \\
\hline SA25 & 294 & $3 / 15 / 73$ & Jemez/Spence Hot Spring & 39.5 & 295 & & & WATSTORE 1993 \\
\hline
\end{tabular}




\begin{tabular}{|c|c|c|c|c|c|c|c|c|}
\hline SITE ID & SAMPLE & DATE & NAME & $\begin{array}{c}T M P \\
C\end{array}$ & $\begin{array}{l}\text { COND } \\
\text { uS/cm }\end{array}$ & $\begin{array}{c}\text { TDS } \\
m g / L\end{array}$ & $\begin{array}{c}\text { FLOW } \\
\text { Lmin }\end{array}$ & REFERENCE \\
\hline SA25 & 293 & $12 / 1 / 72$ & Jemez/Spence Hot Spring & 41.0 & 282 & 224 & 166.54 & WATSTORE 1993 \\
\hline SA25 & VA-83 & $3 / ? / 82$ & Jemez/Spence Hot Spring & 41.5 & 275 & 161 & 160 & Shevenell and others (1987) \\
\hline SA25 & $\mathrm{A}$ & $? / ? / 75-80$ & Jemez/Spence Hot Spring & 42.0 & & 297 & & Swanberg $(1980)$ \\
\hline SA25 & NB82-6 & $? / ? / 80-82$ & Jemez/Spence Hot Spring & 42.0 & & & & Norman and Bernhardt (1982) \\
\hline SA25 & VA-68 & $6 / ? / 81$ & Jemez/Spence Hot Spring & 42.0 & 280 & 297 & 80 & Shevenell and others (1987) \\
\hline SA25 & VA-72 & $10 / ? / 81$ & Jemez/Spence Hot Spring & 42.0 & 275 & 276 & 20 & Shevenell and others (1987) \\
\hline SA25 & VA-120 & $1 / ? / 83$ & Jemez/Spence Hot Spring & 42.3 & 293 & 292 & 160 & Shevenell and others (1987) \\
\hline SA25 & VA-105 & $9 / 2 / 82$ & Jemez/Spence Hot Spring & 42.5 & 315 & 161 & & Shevenell and others (1987) \\
\hline SA25 & 291 & $8 / 1 / 47$ & Jemez/Spence Hot Spring & 44.0 & 283 & & & WATSTORE 1993 \\
\hline SA25 & VA-1 & $7 / ? / 78$ & Jemez/Spence Hot Spring & 45.0 & & 294 & 60 & Shevenell and others (1987) \\
\hline SA26 & 387 & $8 / 31 / 24$ & Jemez/Sulphur Springs Men's Bathhouse & 43.5 & & 7887 & & WATSTORE 1993 \\
\hline SA26 & 392 & $12 / 2 / 74$ & Jemez/Sulphur Springs Men's Bathhouse & 70.0 & & & & WATSTORE 1993 \\
\hline SA26 & VA-75 & $1 / ? / 82$ & Jemez/Sulphur Springs Men's Bathhouse & 72.0 & & & & Shevenell and others (1987) \\
\hline SA26 & VA-81 & $3 / 2 / 82$ & Jemez/Sulphur Springs Men's Bathhouse & 73.6 & 1300 & 5.51 & & Shevenell and others (1987) \\
\hline SA26 & VA-13 & $7 / 2 / 79$ & Jemez/Sulphur Springs Men's Bathhouse & 78.0 & 4050 & 822 & & Shevenell and others (1987) \\
\hline SA26 & 391 & $7 / 21 / 67$ & Jemez/Sulphur Springs Men's Bathhouse & 81.0 & 17300 & & & WATSTORE 1993 \\
\hline SA26 & S-7-80 & $9 / ? / 80$ & Jemez/Sulphur Springs Men's Bathhouse & 82.0 & 10300 & 2597 & & Shevenell and others (1987) \\
\hline SA26 & 389 & $11 / 4 / 63$ & Jemez/Sulphur Springs Men's Bathhouse & 87.0 & 13800 & & & WATSTORE 1993 \\
\hline SA27 & 238 & $8 / 30 / 62$ & Jemez Pueblo Indian Hot Spring & 35.0 & 5680 & & 7.57 & WATSTORE 1993 \\
\hline SA27 & 88TDI10 & $8 / ? / 88$ & Jemez Pueblo Indian Hot Spring & 36.0 & & 3176 & & Witcher $(1988 b)$ \\
\hline SA27 & 88TDI9 & $8 / 2 / 88$ & Jemez Pueblo Indian Hot Spring & 43.0 & & 3572 & & Witcher (1988b) \\
\hline SA28 & 287 & $1 / 16 / 73$ & Jemez/McCauley Hot Spring & 30 & 140 & & 1313.4 & WATSTORE 1993 \\
\hline SA28 & 289 & $12 / 13 / 74$ & Jemez/McCauley Hot Spring & 31 & 165 & & & WATSTORE 1993 \\
\hline SA28 & VA-3 & $7 / 2 / 78$ & Jemez/McCauley Hot Spring & 31.0 & & 189 & 140 & Shevenell and others (1987) \\
\hline SA28 & 286 & $1 / 16 / 73$ & Jemez/McCauley Hot Spring & 31.5 & 165 & & 1392.9 & WATSTORE 1993 \\
\hline SA28 & VA-87 & $3 / ? / 82$ & Jemez/McCauley Hot Spring & 31.5 & 190 & & 400 & Shevenell and others (1987) \\
\hline SA28 & VA-119 & $1 / 2 / 83$ & Jemez/McCauley Hot Spring & 31.9 & 173 & 186 & 960 & Shevenell and others (1987) \\
\hline SA28 & $\mathrm{B}$ & $? / ? / 75-80$ & Jemez/McCauley Hot Spring & 32.0 & & 220 & & Swanberg $(1980)$ \\
\hline SA28 & 285 & $8 / 1 / 47$ & Jemez/McCauley Hot Spring & 43.0 & 198 & & & WATSTORE 1993 \\
\hline SA29 & 424 & $5 / 16 / 73$ & Jemez/San Antonio Hot Springs & 40 & 110 & & 1222.6 & WATSTORE 1993 \\
\hline SA29 & VA-96 & $3 / ? / 82$ & Jemez/San Antonio Hot Spring & 40.8 & 140 & 168 & 125 & Shevenell and others (1987) \\
\hline SA29 & VA-128 & $3 / 2 / 83$ & Jemez'San Antonio Hot Spring & 41.3 & 127 & 167 & & Shevenell and others (1987) \\
\hline SA29 & NB82-5 & $? / ? / 80-82$ & Jemez/San Antonio Hot Spring & 42.0 & & & & Norman and Bernhardt (1982) \\
\hline SA29 & VA-4 & $7 / ? / 78$ & Jemez/San Antonio Hot Spring & 42.0 & 150 & 170 & 150 & Shevenell and others (1987) \\
\hline SA29 & $F$ & $? / ? / 75-80$ & Jemez/San Antonio Hot Spring & 56.0 & & 148 & & Swanberg (1980) \\
\hline SA30 & $5-4-80$ & $9 / 2 / 80$ & Jemez/Sulphur Springs Footbath Spring & 33.0 & 30200 & 8310 & & Shevenell and others (1987) \\
\hline SA30 & 399 & $8 / 31 / 49$ & Jemez/Sulphur Springs Footbath Spring & 40.5 & 4370 & 1730 & & WATSTORE 1993 \\
\hline SA31 & 434 & $8 / 1 / 47$ & Jemez/San Antonio Warm Springs & 38.5 & 167 & & 94.63 & WATSTORE 1993 \\
\hline SA32 & VA-5 & $7 / 2 / 78$ & Jemez/Soda Dam Grotto Spring & 38.0 & & 3950 & 12 & Shevenell and others (1987) \\
\hline
\end{tabular}




\begin{tabular}{|c|c|c|c|c|c|c|c|c|}
\hline SITE ID & SAMPLE & DATE & NAME & $\begin{array}{c}T M P \\
C \\
\end{array}$ & $\begin{array}{l}\text { COND } \\
\text { uS/cm }\end{array}$ & $\begin{array}{c}\text { TDS } \\
\mathrm{mg} / \mathrm{L}\end{array}$ & $\begin{array}{l}\text { FLOW } \\
\text { L/min }\end{array}$ & REFERENCE \\
\hline SA33 & VA-94 & $3 / ? / 82$ & Jemez/Bathhouse HS & 37.4 & 178 & 261 & 18 & Shevenell and others (1987) \\
\hline SA33 & VA-20 & $2 / ? / 79$ & Jemez/Bathhouse HS & 38.0 & 163 & 220 & 12 & Shevenell and others (1987) \\
\hline SA33 & VA-126 & $3 / ? / 83$ & Jemez/Bathhouse HS & 38.1 & 166 & 498 & & Shevenell and others (1987) \\
\hline SA34 & S-5-80 & $9 / 2 / 80$ & Jemez/Sulphur Springs Electric Spring & 36.0 & 12800 & 4580 & 0.5 & Shevenell and others (1987) \\
\hline SA34 & 396 & $8 / 31 / 49$ & Jemez/Sulphur Springs Electric Spring & 39.0 & 12700 & 3160 & & WATSTORE 1993 \\
\hline SA35 & VA-2 & $7 / ? / 78$ & Jemez/Little Spence Hot Spring & 34.0 & & 321 & & Shevenell and others (1987) \\
\hline SA36 & 436 & $1 / 23 / 78$ & Star Lake \#2 Ojo Encino Well & 33 & 3000 & 2190 & 757 & WATSTORE 1993 \\
\hline SA37 & VA-27 & $5 / ? / 79$ & Jemez/Soda Dam Hidden Warm Spring & 29.0 & 5700 & 3990 & 2 & Shevenell and others (1987) \\
\hline SA37 & VA-90 & $3 / ? / 82$ & Jemez/Soda Dam Hidden Warm Spring & 32.0 & 6000 & & 6 & Shevenell and others (1987) \\
\hline SA37 & VA-141 & $2 / 2 / 84$ & Jemez/Soda Dam Hidden Warm Spring & 32.2 & 6260 & 4020 & & Shevenell and others (1987) \\
\hline$\widehat{S A 37}$ & VA-110 & $1 / 2 / 83$ & Jemez/Soda Dam Hidden Warm Spring & 32.3 & 6150 & 3930 & 8 & Shevenell and others (1987) \\
\hline SA38 & 228 & $8 / 26 / 72$ & well & 32 & 3140 & 2460 & & WATSTORE 1993 \\
\hline SA39 & 88TD|4 & $8 / ? / 88$ & Penasco \#3 Spring & 27.0 & & 9672 & & Witcher (1988a) \\
\hline SA40 & 88TDI6 & $8 / 2 / 88$ & Penasco \#4 Spring & 27.0 & & 9924 & & Witcher (1988a) \\
\hline SA41 & 88TDI8 & $8 / ? / 88$ & Salado Warm Spring & 25.0 & & 9608 & & Witcher (1988a) \\
\hline SA41 & M77-SA2 & $? / ? / 77$ & Salado Warm Spring & 25.0 & & 10465 & & Mariner and others (1977) \\
\hline SA42 & VA-130 & $5 / 2 / 83$ & San Ysidro Warm Spring & 22.0 & 9400 & 6810 & & Shevenell and others (1987) \\
\hline SA42 & NB82-9 & $? / ? / 80-82$ & San Ysidro Warm Spring & 24.0 & & & & Norman and Bernhardt (1982) \\
\hline SA42 & VA-33 & $8 / ? / 79$ & San Ysidro Warm Spring & 27.0 & 11550 & 7170 & 1 & Shevenell and others (1987) \\
\hline SA42 & VA-148 & $4 / 2 / 84$ & San Ysidro Warm Spring & & 10000 & & & Shevenell and others (1987) \\
\hline SA43 & 88TD13 & $8 / 2 / 88$ & Swimming Pool Spring & 24.0 & & 7420 & & Witcher (1988b) \\
\hline SA44 & PC2-6 & $9 / 2 / 84$ & Jemez/PC-2 at $1335 \mathrm{ft}$ (w/drilling fluid) & 40.0 & 4980 & 5577 & & Shevenell and others (1987) \\
\hline SA44 & PC1-1 & $4 / ? / 84$ & Jemez/PC-1 at $1712 \mathrm{ft}$ (w/drilling fluid) & & 9500 & 7510 & & Shevenell and others (1987) \\
\hline SF1 & 401 & $7 / 19 / 51$ & Guaje \#3 Well & 30.5 & 192 & & 2407.3 & WATSTORE 1993 \\
\hline SF2 & LA-7 & $9 / 2 / 78$ & Los Alamos \#1B Well & 30.0 & & 559 & 2180 & Shevenell and others (1987) \\
\hline SF2 & 359 & $3 / 16 / 60$ & Los Alamos \#1B Well & 30.5 & 743 & 522 & & WATSTORE 1993 \\
\hline SF2 & 364 & $1 / 15 / 61$ & Los Alamos \#1B Well & 30.5 & 729 & & 1816.8 & WATSTORE 1993 \\
\hline SF2 & 365 & $1 / 15 / 61$ & Los Alamos \#1B Well & 30.5 & 729 & & 1816.8 & WATSTORE 1993 \\
\hline SF2 & 366 & $1 / 19 / 61$ & Los Alamos \#1B Well & 30.5 & 720 & & 1816.8 & WATSTORE 1993 \\
\hline SF2 & 367 & $2 / 8 / 61$ & Los Alamos \#1B Well & 30.5 & 775 & & 2271 & WATSTORE 1993 \\
\hline SF2 & 362 & $1 / 10 / 61$ & Los Alamos \#1B Well & 31 & 766 & & 1816.8 & WATSTORE 1993 \\
\hline SF2 & 363 & $1 / 10 / 61$ & Los Alamos \#1B Well & 31 & 766 & & 1816.8 & WATSTORE 1993 \\
\hline SF2 & 361 & $8 / 31 / 60$ & Los Alamos \#1B Well & 31.5 & 778 & & & WATSTORE 1993 \\
\hline SF2 & 368 & $6 / 9 / 61$ & Los Alamos \#1B Well & 32.0 & 876 & & 2271 & WATSTORE 1993 \\
\hline SF3 & LA-12 & $9 / 2 / 78$ & Los Alamos \#G6 Well & 30.5 & & 191 & 1100 & Shevenell and others (1987) \\
\hline SF4 & 301 & $9 / 26 / 51$ & Los Alamos \#6 Well & 30.0 & 504 & & 2259.7 & WATSTORE 1993 \\
\hline SF4 & 305 & $6 / 25 / 54$ & Los Alamos \#6 Well & 30.0 & 513 & & & WATSTORE 1993 \\
\hline SF4 & 326 & $6 / 19 / 58$ & Los Alamos \#6 Well & 30.0 & 517 & & 2271 & WATSTORE 1993 \\
\hline SF4 & 349 & $7 / 7 / 59$ & Los Alamos \#6 Well & 30.0 & 529 & & 2214.2 & WATSTORE 1993 \\
\hline
\end{tabular}




\begin{tabular}{|c|c|c|c|c|c|c|c|c|}
\hline SITE ID & SAMPLE & DATE & NAME & $\begin{array}{c}T M P \\
C\end{array}$ & $\begin{array}{l}\text { COND } \\
\text { uS/cm }\end{array}$ & $\begin{array}{c}\text { TDS } \\
\mathrm{mg} / \mathrm{L}\end{array}$ & \begin{tabular}{|l|} 
FLOW \\
L/min
\end{tabular} & REFERENCE \\
\hline SF4 & 355 & $6 / 9 / 61$ & Los Alamos \#6 Well & 30.5 & $6 \overline{35}$ & & 22271 & WATSTORE 1993 \\
\hline SF5 & LA-16 & $9 / ? / 78$ & Los Alamos \#G2 Well & 30.0 & & 256 & 1820 & Shevenell and others (1987) \\
\hline SI1 & 118 & $2 / 9 / 39$ & TorC well & 45.5 & 4380 & & 3.79 & WATSTORE 1993 \\
\hline $\mathrm{S} 12$ & SNM-3 & $5 / 31 / 87$ & TorC Artesian well & 41.2 & 5140 & 2697.5 & & Shevenell (1987) \\
\hline $\mathrm{SI2}$ & NB82-19 & $? / ? / 80-82$ & TorC artesian well & 45.0 & & & & Norman and Bernhardt (1982) \\
\hline S13 & 89 & $? / ? / 75-80$ & TorC Blackstone Mineral Bath & 45.0 & & 2608 & & Swanberg (1980) \\
\hline $\mathrm{SI}$ & 112 & $2 / 9 / 39$ & TorC well & 44.5 & 4400 & & 189.25 & WATSTORE 1993 \\
\hline $\mathrm{SI5}$ & NB82-18 & $? / ? / 80-82$ & TorC Sierra Grande Bath & 44.0 & & & & Norman and Bernhardt (1982) \\
\hline S16 & GS38930 & $4 / 15 / 58$ & TorC Yucca Lodge well $14 \mathrm{ft}$ & 40.0 & 4460 & & & Summers (1976) \\
\hline SI6 & GS44734 & $4 / 4 / 60$ & TorC Yucca Lodge well $14 \mathrm{ft}$ & 40.0 & 4450 & & & Summers (1976) \\
\hline S16 & S76TRC14B & $12 / 4 / 74$ & TorC Yucca Lodge well $14 \mathrm{ft}$ & 40.0 & 4500 & & & Summers (1976) \\
\hline SI6 & S76W14-65 & $12 / 14 / 65$ & TorC Yucca Lodge well $14 \mathrm{ft}$ & 40.8 & 4300 & 2621 & & Summers (1976) \\
\hline S16 & GS43047 & $8 / 3 / 59$ & TorC Yucca Lodge well $14 \mathrm{ft}$ & 41.7 & 4450 & & & Summers (1976) \\
\hline SI6 & GS54863 & $8 / 8 / 64$ & TorC Yucca Lodge well $14 \mathrm{ft}$ & 41.7 & 4520 & & 29.15 & Summers (1976) \\
\hline S16 & GS50301 & $3 / 13 / 62$ & TorC Yucca Lodge well $14 \mathrm{ft}$ & 41.9 & 4480 & & & Summers (1976) \\
\hline SI6 & GS38827 & $8 / 5 / 57$ & TorC Yucca Lodge well $14 \mathrm{ft}$ & 42.2 & 4400 & & 32.18 & Summers (1976) \\
\hline SI6 & GS52617 & $8 / 5 / 63$ & TorC Yucca Lodge well $14 \mathrm{ft}$ & 42.2 & 4490 & & & Summers'(1976) \\
\hline S16 & S76TRC14A & $5 / 28 / 54$ & TorC Yucca Lodge well $14 \mathrm{ft}$ & 42.8 & 4510 & 2670 & 4.16 & Summers (1976) \\
\hline SI6 & GS653 & $4 / 28 / 43$ & TorC Yucca Lodge well $14 \mathrm{ft}$ & 43.0 & & & & Summers (1976) \\
\hline S16 & GS18840 & $3 / 31 / 52$ & TorC Yucca Lodge well $14 \mathrm{ft}$ & 43.3 & 4430 & & 7.57 & Summers (1976) \\
\hline SI6 & GS31092 & $8 / 2 / 55$ & TorC Yucca Lodge well $14 \mathrm{ft}$ & 43.3 & 4450 & & 2.91 & Summers (1976) \\
\hline S16 & T41-YLW14 & $2 / ? / 39$ & TorC Yucca Lodge well $14 \mathrm{ft}$ & 43.3 & & & & Summers (1976) \\
\hline SI6 & GS33929 & $9 / 17 / 56$ & TorC Yucca Lodge well $14 \mathrm{ft}$ & 43.6 & 4450 & & 2.91 & Summers (1976) \\
\hline S16 & GS15117 & $12 / 4 / 74$ & TorC Yucca Lodge well $14 \mathrm{ft}$ & & 4500 & & & Summers (1976) \\
\hline SI6 & GS27039 & $7 / 12 / 54$ & TorC Yucca Lodge well $14 \mathrm{ft}$ & & 4420 & & 1.89 & Summers (1976) \\
\hline S17 & NB82-17 & $? / 2 / 80-82$ & TorC Yucca Lodge & 43.0 & & & & Norman and Bernhardt (1982) \\
\hline S18 & S76YUCCA & $12 / 14 / 65$ & TorC Yucca Lodge outdoor pool & 41.7 & & & & Summers (1976) \\
\hline S19 & $\mathrm{B} 10$ & $? / ? / 75-80$ & TorC Sierra Mineral Bath & 41.0 & & 2688 & & Swanberg (1980) \\
\hline S110 & B11 & $? / ? / 75-80$ & TorC warm spring & 41.0 & & 2640 & & Swanberg (1980) \\
\hline S111 & B19 & $? / 2 / 75-80$ & TorC Yucca Lodge & 41.0 & & 2708 & & Swanberg (1980) \\
\hline S112 & 111 & $9 / 15 / 44$ & TorC Old Government Spring & 40.0 & 4480 & & 4.92 & WATSTORE 1993 \\
\hline $\mathrm{S} \mid 12$ & GS20949 & $2 / 9 / 39$ & TorC Old Government Spring & & 4520 & 2560 & & Summers (1976) \\
\hline SI12 & GS684 & $6 / 23 / 38$ & TorC Old Government Spring & & 4590 & & & Summers (1976) \\
\hline $\mathrm{SI13}$ & 107 & $7 / 19 / 45$ & Torc well & 40.0 & 4410 & & 3.03 & WATSTORE 1993 \\
\hline SI14 & 108 & $2 / 9 / 39$ & Torc well & 40 & & & & WATSTORE 1993 \\
\hline $\mathrm{S} \mid 14$ & 113 & $2 / 9 / 39$ & Torc well & 44.5 & 4410 & 2490 & 257.38 & WATSTORE 1993 \\
\hline Sl15 & 98 & $2 / 9 / 39$ & TorC Ponce De Leon Spring & 39 & 4400 & 2440 & 473.13 & WATSTORE 1993 \\
\hline S115 & 96 & $4 / 18 / 50$ & TorC Ponce De Leon Spring & 40.0 & 4480 & & 605.6 & WATSTORE 1993 \\
\hline SI15 & 97 & $4 / 18 / 50$ & TorC Ponce De Leon Spring & 40.0 & 4470 & & & WATSTORE 1993 \\
\hline
\end{tabular}




\begin{tabular}{|c|c|c|c|c|c|c|c|c|}
\hline SITE ID & SAMPLE & DATE & NAME & $\begin{array}{c}T M P \\
C\end{array}$ & $\begin{array}{l}\text { COND } \\
\text { uS/cm }\end{array}$ & $\begin{array}{c}\text { TDS } \\
m g / L\end{array}$ & $\begin{array}{c}\text { FLOW } \\
\text { L/min }\end{array}$ & REFERENCE \\
\hline$\overline{\text { SI15 }}$ & 100 & $2 / 10 / 39$ & TorC Ponce De Leon Spring & 40.0 & 4350 & & & WATSTORE 1993 \\
\hline SI15 & 102 & $4 / 15 / 58$ & TorC Ponce De Leon Spring & 40.0 & 4460 & & & WATSTORE 1993 \\
\hline S115 & 104 & $12 / 4 / 74$ & TorC Ponce De Leon Spring & 40.0 & 4500 & & & WATSTORE 1993 \\
\hline $\mathrm{S} 115$ & 103 & $8 / 13 / 62$ & TorC Ponce De Leon Spring & 41.5 & 4480 & & & WATSTORE 1993 \\
\hline SI15 & 99 & $10 / 6 / 50$ & TorC Ponce De Leon Spring & 42.0 & 4470 & & 113.55 & WATSTORE 1993 \\
\hline SI15 & 105 & $8 / 5 / 57$ & TorC Ponce De Leon Spring & 42.0 & 4400 & & 32.17 & WATSTORE 1993 \\
\hline SI16 & 106 & $2 / 9 / 39$ & TorC Geronimo (State) Springs & 38.5 & & & & WATSTORE 1993 \\
\hline SI16 & SNM-4 & $5 / 31 / 87$ & TorC Geronimo (State) Springs & 42.0 & 5120 & 2696.6 & & Shevenell (1987) \\
\hline SI16 & GS20948 & $2 / 9 / 39$ & TorC Geronimo (State) Springs & & 4290 & 2418 & & Summers (1976) \\
\hline SI16 & GS3932 & $3 / 20 / 26$ & TorC Geronimo (State) Springs & & & & & Summers (1976) \\
\hline $\mathrm{S} \mid 17$ & NB82-24 & $? / ? / 80-82$ & Hillsboro Warm Spring & 34.0 & & & & Norman and Bernhardt (1982) \\
\hline $\mathrm{S} 117$ & JUST1 & $? / ? / 75-80$ & Hillsboro Warm Spring & 34.5 & & 568 & & Swanberg $(1980)$ \\
\hline SI19 & S76DWS-65 & $12 / 16 / 65$ & Derry Warm Springs & 33.8 & 1420 & 823 & & Summers (1976) \\
\hline$\$ 119$ & S76DWS-74 & $12 / 4 / 74$ & Derry Warm Springs & 33.8 & & & & Summers (1976) \\
\hline 5119 & 124 & $7 / 8 / 55$ & Sun Oil Test Well & 34.0 & 2600 & & 3406.5 & WATSTORE 1993 \\
\hline$S 119$ & B5 & $? / ? / 75-80$ & Derry Warm Springs & & & 1240 & & Swanberg (1980) \\
\hline$S \mid 19$ & $\mathrm{~B} 6$ & $? / ? / 75-80$ & Derry Warm Springs & & & 1228 & & Swanberg (1980) \\
\hline S119 & GS1510 & $12 / 4 / 74$ & Derry Warm Springs & & 1660 & & & Summers (1976) \\
\hline 5120 & CON54DWS & $4 / 17 / 47$ & Derry Warm Springs & 33.9 & 1650 & 1030 & & Summers (1976) \\
\hline $\mathrm{S} 120$ & GS35977 & $4 / 30 / 57$ & Derry Warm Springs & 33.9 & 1660 & 823 & & Summers (1976) \\
\hline $\mathrm{Sl20}$ & 88 & $3 / 7 / 52$ & Derry Warm Springs & 34.0 & 1660 & & 37.85 & WATSTORE 1993 \\
\hline $\mathrm{S} 120$ & 89 & $4 / 30 / 57$ & Derry Warm Springs & 34.0 & 1660 & & & WATSTORE 1993 \\
\hline $\mathrm{SI} 20$ & GS18725 & $3 / 7 / 52$ & Derry Warm Springs & 34.0 & 1660 & & 189 & Summers (1976) \\
\hline$S 121$ & 90 & $4 / 17 / 47$ & well & 34.0 & 1650 & & & WATSTORE 1993 \\
\hline SI21 & 91 & $3 / 7 / 52$ & well & 34.0 & 1660 & & 30.28 & WATSTORE 1993 \\
\hline$S 121$ & 92 & $12 / 4 / 74$ & well & 34 & 1660 & & & WATSTORE 1993 \\
\hline$S 121$ & 125 & $3 / 7 / 52$ & well & 34.0 & 1660 & & & WATSTORE 1993 \\
\hline $\mathrm{S122}$ & 83 & $9 / 9 / 71$ & well & 31 & 1350 & 953 & & WATSTORE 1993 \\
\hline $\mathrm{SI23}$ & S76AFTER & $12 / 15 / 65$ & Barney lorio \#1 Fee & 33.0 & 5600 & 4931 & 88 & Summers (1976) \\
\hline $\mathrm{S123}$ & $\mathrm{B} 12$ & $? / 7 / 75-80$ & warm spring & & & 1392 & & Swanberg (1980) \\
\hline $\mathrm{SI24}$ & HAREMIT & $12 / ? / 1901$ & TorC "warm spring" & & & 2635 & & Summers (1976) \\
\hline$S 125$ & GS44137 & $12 / 2 / 59$ & Barney lorio \#1 Fee & & 5600 & & & Summers (1976) \\
\hline SJ1 & 440 & $7 / 22 / 78$ & well & 62 & 11400 & & 1514 & WATSTORE 1993 \\
\hline SJ2 & 442 & $1 / 6 / 76$ & well & 48 & 4000 & 3620 & & WATSTORE 1993 \\
\hline SJ2 & 441 & $5 / 5 / 75$ & well & 57 & 4350 & & 1135.5 & WATSTORE 1993 \\
\hline SJ3 & 448 & $6 / 11 / 87$ & Navajo well & 51.8 & 1200 & & & WATSTORE 1993 \\
\hline SJ3 & 446 & $9 / 24 / 73$ & Navajo well & 61 & 1390 & 880 & & WATSTORE 1993 \\
\hline SJ4 & 443 & $3 / 28 / 78$ & Dome Well Chaco & 42 & 10000 & & & WATSTORE 1993 \\
\hline SJ5 & 474 & $7 / 21 / 87$ & ARCO WS-2 well & 39.9 & 8000 & & & WATSTORE 1993 \\
\hline
\end{tabular}




\begin{tabular}{|c|c|c|c|c|c|c|c|c|}
\hline SITEID & SAMPLE & DATE & NAME & $\begin{array}{c}T M P \\
C \\
\end{array}$ & $\begin{array}{l}\text { COND } \\
u S / \mathrm{cm}\end{array}$ & $\begin{array}{c}T D S \\
m g / L\end{array}$ & $\begin{array}{l}\text { FLOW } \\
\text { L/min }\end{array}$ & REFERENCE \\
\hline $5 \sqrt{6} 6$ & 444 & $9 / 12 / 49$ & Navajo well & $35 . \overline{5}$ & 2320 & & $\overline{75.7}$ & WATSTORE 1993 \\
\hline SJ7 & 466 & $6 / 10 / 87$ & Navajo 12T-630 Well & 31.0 & 4930 & & & WATSTORE 1993 \\
\hline SJ7 & 464 & $6 / 19 / 86$ & Navajo 12T-630 Well & 33.0 & 6000 & & & WATSTORE 1993 \\
\hline SJ8 & 437 & $4 / 22 / 86$ & well & 32.8 & 2720 & & & WATSTORE 1993 \\
\hline $5 \mathrm{~J} 8$ & 438 & $4 / 22 / 86$ & well & 32.8 & 2720 & & & WATSTORE 1993 \\
\hline SJ8 & 439 & $10 / 21 / 87$ & well & 32.9 & 2800 & & & WATSTORE 1993 \\
\hline SJ9 & 471 & $8 / 29 / 49$ & Navajo well & 30.5 & 5050 & & 567.75 & WATSTORE 1993 \\
\hline Sj9 & 473 & $10 / 9 / 52$ & Navajo well & 32.0 & 4110 & & 567.75 & WATSTORE 1993 \\
\hline SJ10 & 467 & $3 / 9 / 61$ & Navajo 12T-520 Well & 30.0 & 4050 & & 586.68 & WATSTORE 1993 \\
\hline SJ10 & 468 & $6 / 16 / 86$ & Navajo 12T-520 Well & 31.0 & 4000 & & & WATSTORE 1993 \\
\hline SJ10 & 470 & $6 / 9 / 87$ & Navajo12T-520 Well & 31.1 & 4300 & & & WATSTORE 1993 \\
\hline SJ11 & 453 & $4 / 15 / 54$ & well & 30.5 & 8610 & & & WATSTORE 1993 \\
\hline SJ12 & 463 & $6 / 10 / 87$ & Navajo 12T-629 Well & 30.5 & 4200 & & & WATSTORE 1993 \\
\hline SM1 & S76MNTZ1 & $1 / 3 / 66$ & Montezuma Hot Spring \#1 & 55.2 & 810 & & & Summers (1976) \\
\hline SM1 & GS2083 & $5 / 16 / 39$ & Montezuma Hot Spring \#1 & & 878 & 554 & & Summers (1976) \\
\hline SM1 & GS4801 & $7 / 2 / 40$ & Montezuma Hot Spring \#1 & & 870 & 537 & & Summers (1976) \\
\hline SM1 & GS5233 & $8 / 20 / 40$ & Montezuma Hot Spring \#1 & & 878 & 531 & & Summers (1976) \\
\hline SM2 & GS18609 & $3 / 11 / 52$ & Montezuma Hot Spring \#6 & 50.6 & 876 & 530 & & Summers (1976) \\
\hline SM3 & NM3 & $? / ? / 75-80$ & Montezuma Hot Spring & 34.3 & & 464 & & Swanberg (1980) \\
\hline SM3 & NM6 & $? / ? / 75-80$ & Montezuma Hot Spring & 35.6 & & 400 & & Swanberg (1980) \\
\hline SM3 & NM2 & $? / ? / 75-80$ & Montezuma Hot Spring & 48.0 & & 452 & & Swanberg (1980) \\
\hline SM3 & NB82-11 & $? / ? / 80-82$ & Montezuma Hot Spring & 49.0 & & & & Norman and Bernhardt (1982) \\
\hline SM3 & NM4 & $? / ? / 75-80$ & Montezuma Hot Spring & 53.0 & & 460 & & Swanberg (1980) \\
\hline SM3 & NM1 & $? / ? / 75-80$ & Montezuma Hot Spring & 53.8 & & 432 & & Swanberg (1980) \\
\hline SM3 & NM5 & $? / ? / 75-80$ & Montezuma Hot Spring & 58.5 & & 448 & & Swanberg (1980) \\
\hline SM4 & GS18610 & $3 / 11 / 52$ & Montezuma Hot Spring \#13 & 41.1 & 876 & 528 & & Summers (1976) \\
\hline SM6 & S76MNTZ16 & $2 / 11 / 66$ & Montezuma Hot Spring \#16 & & & & & Summers (1976) \\
\hline SM9 & S76MNTZ2 & $2 / 11 / 66$ & Montezuma Hot Spring \#2 & & & & & Summers (1976) \\
\hline SM10 & S76MNTZ20 & $2 / 11 / 66$ & Montezuma Hot Spring \#20 & & & & & Summers (1976) \\
\hline SM15 & S76MNTZ15 & $2 / 11 / 66$ & Montezuma Hot Spring \#15 & & & & & Summers (1976) \\
\hline SM18 & S76MNTZ18 & $2 / 11 / 66$ & Montezuma Hot Spring \#18 & & & & & Summers (1976) \\
\hline SM19 & S76MNTZ19 & $2 / 11 / 66$ & Montezuma Hot Spring \#19 & & & & & Summers (1976) \\
\hline S01 & $\mathrm{CH} 823-66$ & $8 / 23 / 66$ & core hole & 42.2 & 3460 & & & Summers (1976) \\
\hline $\mathrm{SO} 2$ & 166 & $5 / 7 / 79$ & warm well & 36 & 430 & & 11.36 & WATSTORE 1993 \\
\hline $\mathrm{SO3}$ & 126 & $8 / 24 / 79$ & Welty Salty Well & 35 & 2100 & 1440 & 18.93 & WATSTORE 1993 \\
\hline SO4 & NB82-16 & $? / ? / 80-82$ & Bosque del Apache Well \#13 & 33.0 & & & & Norman and Bernhardt (1982) \\
\hline SO5 & 127 & $7 / 2 / 80$ & warm well & 33 & 4600 & & & WATSTORE 1993 \\
\hline SO5 & 128 & $2 / 24 / 88$ & warm well & 33.0 & 4450 & 2870 & & WATSTORE 1993 \\
\hline SO6 & 161 & $7 / 24 / 56$ & Blue Canyon Well & 30.0 & 380 & & & WATSTORE 1993 \\
\hline
\end{tabular}




\begin{tabular}{|c|c|c|c|c|c|c|c|c|}
\hline SITE ID & SAMPLE & DATE & NAME & $\begin{array}{c}\text { TMP } \\
C\end{array}$ & $\begin{array}{l}\text { COND } \\
\text { uS/cm }\end{array}$ & $\begin{array}{c}\text { TDS } \\
\mathrm{mg} / \mathrm{L}\end{array}$ & $\begin{array}{c}\text { FLOW } \\
\text { L/min }\end{array}$ & REFERENCE \\
\hline SO6 & 160 & $12 / 4 / 74$ & Blue Canyon Well & 31.5 & 360 & & & WATSTORE 1993 \\
\hline SO6 & 163 & $1 / 22 / 64$ & Blue Canyon Well & 31.8 & 380 & & 15.14 & WATSTORE 1993 \\
\hline SO6 & GS-BLUE & $4 / 10 / 65$ & Blue Canyon Well & 32.2 & 375 & & 18.93 & Summers (1976) \\
\hline SO6 & GS54325 & $1 / 22 / 64$ & Blue Canyon Well & 32.2 & 380 & & 18.93 & Summers (1976) \\
\hline SO6 & 159 & $7 / 24 / 56$ & Blue Canyon Well & 32.4 & 380 & & 75.7 & WATSTORE 1993 \\
\hline SO6 & NB82-12 & $? / ? / 80-82$ & Blue Canyon Well & 33.0 & & & & Norman and Bernhardt (1982) \\
\hline SO6 & GS3372 & $7 / 24 / 56$ & Blue Canyon Well & 33.3 & 380 & & 71.92 & Summers (1976) \\
\hline SO6 & BLUE12-74 & $12 / 4 / 74$ & Blue Canyon Well & & & & 18.93 & Summers (1976) \\
\hline 506 & GS15116 & $12 / 4 / 74$ & Blue Canyon Well & & 360 & & & Summers (1976) \\
\hline SO6 & HALL4 & $12 / 20 / 61$ & Blue Canyon Well & & & & & Summers (1976) \\
\hline S06 & BLUE10-65 & $10 / 22 / 65$ & Blue Canyon Well & & 365 & 145 & & Summers (1976) \\
\hline SO7 & 148 & $1 / 24 / 57$ & Socorro Gallery Spring & 30 & 348 & 224 & & WATSTORE 1993 \\
\hline SO7 & 149 & $3 / 20 / 58$ & Socorro Gallery Spring & 30.0 & 362 & 231 & & WATSTORE 1993 \\
\hline SO7 & 151 & $2 / 5 / 63$ & Socorro Gallery Spring & 30.0 & 356 & & & WATSTORE 1993 \\
\hline SO7 & 155 & $2 / 4 / 77$ & Socorro Gallery Spring & 30.0 & 370 & & & WATSTORE 1993 \\
\hline SO7 & 153 & $4 / 10 / 65$ & Socorro Gallery Spring & 30.5 & 346 & & & WATSTORE 1993 \\
\hline SO7 & 154 & $10 / 23 / 65$ & Socorro Gallery Spring & 30.5 & & & & WATSTORE 1993 \\
\hline SO7 & 150 & $12 / 12 / 61$ & Socorro Gallery Spring & 31.0 & 370 & & & WATSTORE 1993 \\
\hline SO7 & 141 & $3 / 20 / 58$ & Socorro Gallery Spring & 31.5 & 362 & & 832.7 & WATSTORE 1993 \\
\hline SO7 & 140 & $1 / 24 / 57$ & Socorro Gallery Spring & 32 & 348 & 224 & 1336.1 & WATSTORE 1993 \\
\hline SO7 & NB82-13 & $? / ? / 80-82$ & Socorro Gallery Spring & 32.0 & & & & Norman and Bernhardt (1982) \\
\hline SO7 & S76FIG27-3 & $10 / 23 / 65$ & Socorro Gallery Spring & 32.4 & 334 & 245 & & Summers $(1976)$ \\
\hline SO7 & 157 & $10 / 30 / 80$ & Socorro Gallery Spring & 32.5 & 352 & 224 & & WATSTORE 1993 \\
\hline SO7 & S76FIG27-1 & $10 / 23 / 65$ & Socorro Gallery Spring & 32.6 & 335 & 249 & & Summers (1976) \\
\hline S07 & WALD1956 & $? / ? / 52$ & Socorro Gallery Spring & 32.8 & & & & Summers (1976) \\
\hline SO7 & 158 & $1 / 1 / 51$ & Socorro Gallery Spring & 33.0 & & 234 & & WATSTORE 1993 \\
\hline $\mathrm{SO7}$ & HALL1 & $12 / 12 / 61$ & Socorro Gallery Spring & 33.0 & 370 & & & Summers (1976) \\
\hline SO7 & GS410-65 & $4 / 10 / 65$ & Socorro Gallery Spring & 33.1 & 346 & & & Summers (1976) \\
\hline SO7 & S76FIG27-4 & $10 / 23 / 65$ & Socorro Gallery Spring & 33.1 & 339 & 232 & & Summers (1976) \\
\hline 507 & 142 & $4 / 10 / 65$ & Socorro Gallery Spring & 33.5 & 346 & & & WATSTORE 1993 \\
\hline SO7 & S76FIG27-2 & $10 / 23 / 65$ & Socorro Gallery Spring & 33.6 & 340 & 234 & & Summers (1976) \\
\hline SO7 & GS110-64 & $1 / 10 / 64$ & Socorro Gallery Spring & & 356 & 236 & & Summers (1976) \\
\hline SO7 & HALL2 & $2 / 5 / 63$ & Socorro Gallery Spring & & 356 & & & Summers (1976) \\
\hline SO7 & NMHSS-SC & $6 / 19 / 73$ & Socorro Gallery Spring & & 330 & 220 & & Summers (1976) \\
\hline SO7 & S76GS2-48 & $2 / 10 / 48$ & Socorro Gallery Spring * & & 352 & & & Summers (1976) \\
\hline SO7 & SCOFLD2 & $12 / 4 / 36$ & Socorro Gallery Spring & & 347 & & & Summers (1976) \\
\hline SO7 & CLKPRST & $5 / 24 / 31$ & Socorro Gallery Spring & & & 318 & & Summers (1976) \\
\hline SO7 & GS38854 & $3 / 20 / 58$ & Socorro Gallery Spring & & 362 & & 832.79 & Summers (1976) \\
\hline SO7 & JONESO4 & $? / ? / 03$ & Socorro Gallery Spring & & & & 1870 & Summers (1976) \\
\hline
\end{tabular}




\begin{tabular}{|c|c|c|c|c|c|c|c|c|}
\hline SITE ID & SAMPLE & DATE & NAME & $\begin{array}{c}T M P \\
C\end{array}$ & $\begin{array}{l}\text { COND } \\
u S / \mathrm{cm}\end{array}$ & $\begin{array}{c}\text { TDS } \\
m g / L\end{array}$ & \begin{tabular}{|l|} 
FLOW \\
L/min
\end{tabular} & REFERENCE \\
\hline S07 & SCOFLD1 & $2 / 17 / 36$ & Socorro Gallery Spring & & 340 & & & Summers (1976) \\
\hline SO7 & SCOFLD3 & $12 / 4 / 36$ & Socorro Gallery Spring & & 348 & & & Summers (1976) \\
\hline SO7 & SCTTBRK & $1 / 24 / 57$ & Socorro Gallery Spring & & 348 & 224 & 1184.8 & Summers (1976) \\
\hline SO7 & SETTLING & $10 / 23 / 65$ & Socorro Gallery Spring & & & 232 & 1105.3 & Summers (1976) \\
\hline SO8 & 133 & $1 / 22 / 64$ & Socorro/Sedilla Gallery Spring & 30 & 352 & 237 & & WATSTORE 1993 \\
\hline S08 & 139 & $9 / 4 / 80$ & Socorro/Sedilla Gallery Spring & 30.0 & 331 & 255 & & WATSTORE 1993 \\
\hline SO8 & 137 & $7 / 1 / 77$ & Socorro/Sedilla Gallery Spring & 30.5 & 340 & & & WATSTORE 1993 \\
\hline $\mathrm{SO8}$ & HALL3 & $12 / 12 / 61$ & Socorro/Sedilla Gallery Spring & 31.1 & 370 & & & Summers (1976) \\
\hline $\mathrm{SO8}$ & 138 & $1 / 22 / 64$ & Socorro/Sedilla Gallery Spring & 32 & 352 & & 643.45 & WATSTORE 1993 \\
\hline SO8 & SNM-21 & $6 / 5 / 87$ & Socorro/Sedilla Gallery Spring & 32.0 & 338 & 319.6 & & Shevenell (1987) \\
\hline SO8 & GS54324 & $1 / 22 / 64$ & Socorro/Sedilla Gallery Spring & 32.2 & 352 & & & Summers (1976) \\
\hline SO8 & $\mathrm{B} 13$ & $? / 2 / 75-80$ & Socorro/Sedilla Gallery Spring & 34.0 & & 284 & & Swanberg (1980) \\
\hline SO8 & GS38853 & $3 / 20 / 58$ & Socorro/Sedilla Gallery Spring & & & & 908.49 & Summers (1976) \\
\hline S08 & NMHSS-SD & $6 / 19 / 73$ & Socorro/Sedilla Gallery Spring & & 343 & 298 & & Summers (1976) \\
\hline 508 & S76SEDI & $10 / 23 / 65$ & Socorro/Sedilla Gallery Spring & & 336 & 249 & & Summers (1976) \\
\hline SO9 & $\mathrm{B} 28$ & $? / ? / 75-80$ & well & 30.0 & & 192 & & Swanberg (1980) \\
\hline SO10 & 167 & $7 / 10 / 79$ & well & 30.0 & 4000 & 4220 & & WATSTORE 1993 \\
\hline S011 & $\mathrm{B25}$ & $? / ? / 75-80$ & Monticello Box Warm Spring & 29.0 & & 516 & & Swanberg (1980) \\
\hline S012 & $\mathrm{B} 17$ & $? / ? / 75-80$ & Monticello Box Warm Spring & 28.0 & & 468 & & Swanberg (1980) \\
\hline S013 & LUC-17 & $5 / ? / 80$ & artesian well & 26 & 4000 & & 10 & Goff and others (1983) \\
\hline S014 & LUC-19 & $5 / 2 / 80$ & Field Artesian Well & 25 & 3700 & & 40 & Goff and others (1983) \\
\hline S015 & LUC-25 & $5 / ? / 80$ & Ojo Saladito Spring & 24 & 12000 & & 20 & Goff and others (1983) \\
\hline SP16 & B14 & $? / ? 775-80$ & Cook Spring & & & 348 & & Swanberg (1980) \\
\hline TS1 & S76JDUN & $12 / 4 / 65$ & Hondo Hot Spring & 36.9 & 740 & 505 & 1.89 & Summers (1976) \\
\hline TS1 & NM31 & $? / ? / 75-80$ & Hondo Hot Spring & 40.6 & & 584 & & Swanberg (1980) \\
\hline TS2 & NM30 & $? / ? / 75-80$ & Mamby Hot Spring & 32.8 & & 396 & & Swanberg (1980) \\
\hline TS2 & 458 & $12 / 3 / 74$ & Mamby Hot Spring & 34 & 794 & & & WATSTORE 1993 \\
\hline TS2 & S76MAM3 & $12 / 3 / 74$ & Mamby Hot Spring & 34.4 & & & & Summers (1976) \\
\hline TS2 & S76MAM1 & $12 / 3 / 65$ & Mamby Hot Spring & 37.8 & 660 & 520 & 113 & Summers (1976) \\
\hline TS2 & S76MAM2 & $12 / 3 / 65$ & Mamby Hot Spring & 37.8 & 729 & 491 & 113 & Summers (1976) \\
\hline TS2 & 457 & $7 / 21 / 67$ & Mamby Hot Spring & 38 & 736 & 504 & & WATSTORE 1993 \\
\hline TS2 & 459 & $7 / 21 / 67$ & Mamby Hot Spring & 38 & 736 & 491 & & WATSTORE 1993 \\
\hline TS2 & NB82-1 & $? / ? / 80-82$ & Mamby Hot Spring & 38.0 & & & & Norman and Bernhardt (1982) \\
\hline TS2 & NM29 & $? / ? / 75-80$ & Mamby Hot Spring & 38.3 & & 552 & & Swanberg (1980) \\
\hline TS2 & GS15113 & $12 / 3 / 74$ & Mamby Hot Spring & & 794 & & & Summers (1976) \\
\hline TS3 & 460 & $7 / 22 / 76$ & warm spring & 37 & 760 & & & WATSTORE 1993 \\
\hline TS4 & 451 & $12 / 3 / 74$ & Rancho Del Rio Grande Well & 32 & 786 & & & WATSTORE 1993 \\
\hline TS5 & NB82-2 & $? / ? / 80-82$ & Ponce de Leon Hot Spring & 34.0 & & & & Norman and Bernhardt (1982) \\
\hline TS5 & S76SITE6 & $12 / 3 / 74$ & Ponce de Leon Hot Spring & 34.0 & & & & Summers (1976) \\
\hline
\end{tabular}




\begin{tabular}{|c|c|c|c|c|c|c|c|c|}
\hline SITE ID & SAMPLE & DATE & NAME & $\begin{array}{c}\text { TMP } \\
\text { C }\end{array}$ & $\begin{array}{c}\text { COND } \\
\text { uS/cm }\end{array}$ & $\begin{array}{c}\text { TDS } \\
\mathrm{mg} / \mathrm{L}\end{array}$ & $\begin{array}{c}\text { FLOW } \\
\text { L/min }\end{array}$ & REFERENCE \\
\hline \hline TS5 & NM22 & ?/7/75-80 & Ponce de Leon Hot Spring & 34.4 & & 512 & & Swanberg (1980) \\
\hline TS5 & S76SITE2 & $12 / 5 / 65$ & Ponce de Leon Hot Spring & 34.6 & 740 & 486 & 529.95 & Summers (1976) \\
\hline TS5 & GS15114 & $12 / 3 / 74$ & Ponce de Leon Hot Spring & & 786 & & & Summers (1976) \\
\hline VA1 & S76-52270 & $5 / 22 / 70$ & well & 80.0 & 3450 & 3440 & & Summers (1976) \\
\hline VA2 & 172 & $7 / 13 / 75$ & well & 32.5 & 625 & 468 & & WATSTORE 1993 \\
\hline
\end{tabular}




\section{APPENDIX 5}

REFERENCES FOR DATA SOURCES 


\section{COMPILED DATA BASE REFERENCES}

Callender, J. F., 1985, Evaluation of geothermal potential of Rio Grande rift and Basin and Range province, New Mexico: New Mexico Energy Research and Development Institute Report NMERDI 2-2164, p. 263.

Cunniff, R. A., Houghton, C., Archey, J., Ferguson, E., Clanton, M., 1981, New Mexico State University geothermal production well completion and testing, NMSU-PG-3 (LRG-520): NMSU Physical Science Laboratory Technical Completion Report, 23 p.

Cunniff, R. A., Houghton, C., and Clanton, M., 1982, Testing and repair, NMSU geothermal disposal well (old golf course well): NMSU Physical Science Laboratory Technical Completion Report, $41 \mathrm{p}$.

Cunniff, R. A., Houghton, C., and Chintawongvanich, P., 1983, New Mexico State University geothermal reinjection well completion and testing, NMSU-GD-2 (LRG-3648): NMSU Physical Science Laboratory Technical Completion Report, $15 \mathrm{p}$.

Cunniff, R. A., and Chintawongvanich, P., 1985; New Mexico State University geothermal exploratory well, DT-3: NMSU Physical Science Laboratory Technical Completion Report, 129 p.

Cunniff, R. A., Fisher, K. P., and Chintawongvanich, P., 1984, New Mexico State University campus geothermal demonstration: NMSU Physical Science Laboratory Technical Completion Report, 264 p.

Elston, W. E., 1981, Assessment of the geothermal potential of southwestern New Mexico: New Mexico Research and Development Institute Report 2-67$2133,39 \mathrm{p}$.

Elston, W. E., Deal, E. G., and Logsdon, M. J., 1983, Geology and geothermal waters of Lightning Dock region, Animas Valley and Pyramid Mountains, Hidalgo County, New Mexico: New Mexico Bureau of Mines and Mineral Resources Circular 177, $44 \mathrm{p}$.

Goff, F. E., McComick, T., Gardner, J. N., Trujillo, P. E., Counce, D., Vidale, R., Charles, R., 1983, Water chemistry of the Lucero uplift, New Mexico: Los Alamos National Laboratory Report LA-9738-OBES, 26 p.

Goff, F. E., 1991, Letter: Files, Southwest Technology Development Institute, New Mexico State University. 
Goff, F. E., McCormick, T., Trujillo, P. E., Counce, D., and Grigsby, C. O., 1982, Geochemical data for 95 thermal and nonthermal waters of the Valles Caldera southern Jemez Mountains region, New Mexico: Los Alamos National Laboratory Report LA-9367-OBES, $51 \mathrm{p}$.

Gross, J. T., 1988, A hydrogeological investigation of the Las Cruces geothermal field: unpub MS Thesis, New Mexico State University, 212 p.

Henry, C. D., and Gluck, J. K., 1981, A preliminary assessment of the geologic setting, hydrology, and geochemistry of the Hueco Tanks geothermal area, Texas and New Mexico: Texas Bureau of Economic Geology, University of Texas Austin, Geologic Circular 81-1, 48 p.

Hood, J. W., and Kister, L. R., 1962, Saline-water resources of New Mexico: U. S. Geological Survey Water Supply Paper 1601, 70 p.

Icerman, L., and Lohse, R. L., 1983, Geothermal low-temperature reservoir assessment in Dona Ana County, New Mexico: New Mexico Energy Research and Development Institute Report NMERDI 2-69-2202, 188 p.

Jiracek, G. R., 1983, Evaluation of the geothermal resource in the area of Albuquerque, New Mexico: New Mexico Research and Development Institute Report NMRDI 2-67-2135, $169 \mathrm{p}$.

Levitte, D. and Gambill, D. T., 1980, Geothermal potential of west-central New Mexico from geochemical and thermal gradient data: Los Alamos Scientific Laboratory Report LA-8608-MS, 102 p.

Mariner, R. H., Presser, T. S., and Evans, W. C., 1977, Chemical, isotopic and gas compositions of selected thermal springs in Arizona, New Mexico, and Utah: U. S. Geological Survey Open-File Report 77-654, 42 p.

McLean, J. S., 1970, Saline ground-water resources of the Tularosa basin, New Mexico: U. S. Department of Interior, Office of Saline Water Research and Development Progress Report 561, $128 \mathrm{p}$.

McLean, J. S., 1977, Hydrologic maps and data in the Mimbres basin, New Mexico: U. S. Geological Survey Open-File Report 77-314, 531 p.

Myers, R. G., 1992, Geohydrology and potential hydrologic effects of surface coal mining of the San Augustine coal area and adjacent areas, Catron and Cibola Counties, New Mexico: U. S. Geological Survey Water Resources Investigation Report 92-4004, $52 \mathrm{p}$. 
Norman, D. L., and Bernhardt, C. A., 1982, Assessment of geothermal reservoirs by analysis of gases in thermal waters: New Mexico Energy Research and Development Institute Report EMD 2-68-2305, 130 p.

Schwab, G. E., Summers, W. K., Colpitts, R. M., Tenten, C. E., and Young, W. K., 1982, Pumping tests of Campbell No. 2, Gila Hot Springs, Grant County, New Mexico: W. K. Summers and Associates, Inc. Report prepared for EG\&G Idaho, Inc, $194 \mathrm{p}$.

Shevenell, L., Goff. F., Vuataz, F., Trujillo, P. E., Counce, D., Janik, C. J., and Evans, W., 1987, Hydrogeological data for thermal and nonthermal waters and gases of the Valles Caldera southern Jemez Mountains region, New Mexico: Los Alamos National Laboratory Report LA-10923-OBES, 100 p.

Shevenell, L., 1987, Letter: Files, Southwest Technology Development Institute, New Mexico State University.

Stix, T., Pearson, C., Vuataz, F. D., Goff, F. E., East, J., and Hoffers, B., 1982, Geology, resistivity, and hydrochemistry of the Ojo Caliente hot springs area, northern, New Mexico: Geothermal Resources Council Transactions, v.6, p. 5558.

Summers, W. K., 1976, Catalog of thermal waters in New Mexico: New Mexico Bureau of Mines and Mineral Resources Hydrologic Report 4, $80 \mathrm{p}$.

Summers, W. K., and Colpitts, R. M., 1980, Preliminary appraisal of the hydrothermal resource potential of the Gila Hot Springs area, Grant and Catron Counties, New Mexico: W. K. Summers and Associates Report prepared for D. A. and Ida Campbell, $102 \mathrm{p}$.

Swanberg, C. A., 1980, Chemistry, origin, and geothermal potential of thermal and non-thermal waters in New Mexico: unpublished report submitted under U. S. Geological Survey Grant 14-08-0001-6-255, 99 p.

Swanberg, C. A., 1976, Geothermal studies in southwest New Mexico: New Mexico Energy Institute Technical Completion Report NMEI 3, 63 p.

Trainer, F. W., and Lyford, F. P. 1979, Geothermal hydrology in the Rio Grande rift, north-central New Mexico, in Ingersoll, R. V., Woodward, L. A., and James, H. L., eds., Sante Fe Country: New Mexico Geological Society 30th Field Conference Guidebook, p. 299-306.

Trauger, F. D., 1972, Water resources and general geology of Grant County, New Mexico: New Mexico Bureau of Mines and Mineral Resources Hydrologic Report 2, $211 \mathrm{p}$. 
Vuataz, F. D., Stix, J., Goff, F., and Pearson, C. F., 1984, Low-temperature geothermal potential of the Ojo Caliente warm springs area, northern New Mexico: Los Alamos National Laboratory Report LA-10105-OBES, 56 p.

White, W. E., and Kues, G. E., 1992, Inventory of springs in the State of New Mexico: U. S. Geological Survey Open-File Report 92-118, 253 p.

Wilson, C. A., White, R. R., Orr, B. R., and Roybal, R. G., 1981, Water resources of the Rincon and Mesilla Valleys and adjacent areas, New Mexico: New Mexico State Engineer Technical Report 43, 514 p.

Witcher, J. C., 1988a, Geothermal resources of the Zia Pueblo, a prefeasibility assessment: Southwest Technology Development Institute, New Mexico State University, Technical Report prepared for the Council of Energy Resource Tribes, $51 \mathrm{p}$.

Witcher, J. C., 1988b, Geothermal resources of the Jemez Pueblo, a prefeasibility assessment: Southwest Technology Development Institute, New Mexico State Univesity, Technical Report prepared for the Council of Energy Resource Tribes, $50 \mathrm{p}$.

Witcher, J. C., 1991a, Jemez Pueblo geothermal assessment: Technology Enterprise Division, New Mexico Economic Development Department Report 278-5206, $11 p$.

Witcher, J. C., 1991b, The Rincon geothermal system, southern Rio Grande rift, New Mexico: a preliminary report on a recent discovery: Transactions, Geothermal Resources Council, v. 15, p. 205-212.

Witcher, J. C., Heller-Turietta, M., Fischer, C., 1992, Geothermal resources at the Pueblo of Jemez: Southwest Technology Development Institute, New Mexico State University, Technical Report prepared for Council of Energy Resource Tribes, $36 \mathrm{p}$. 
APPENDIX 6

NEW MEXICO WELL AND SPRING LOCATION SYSTEM 


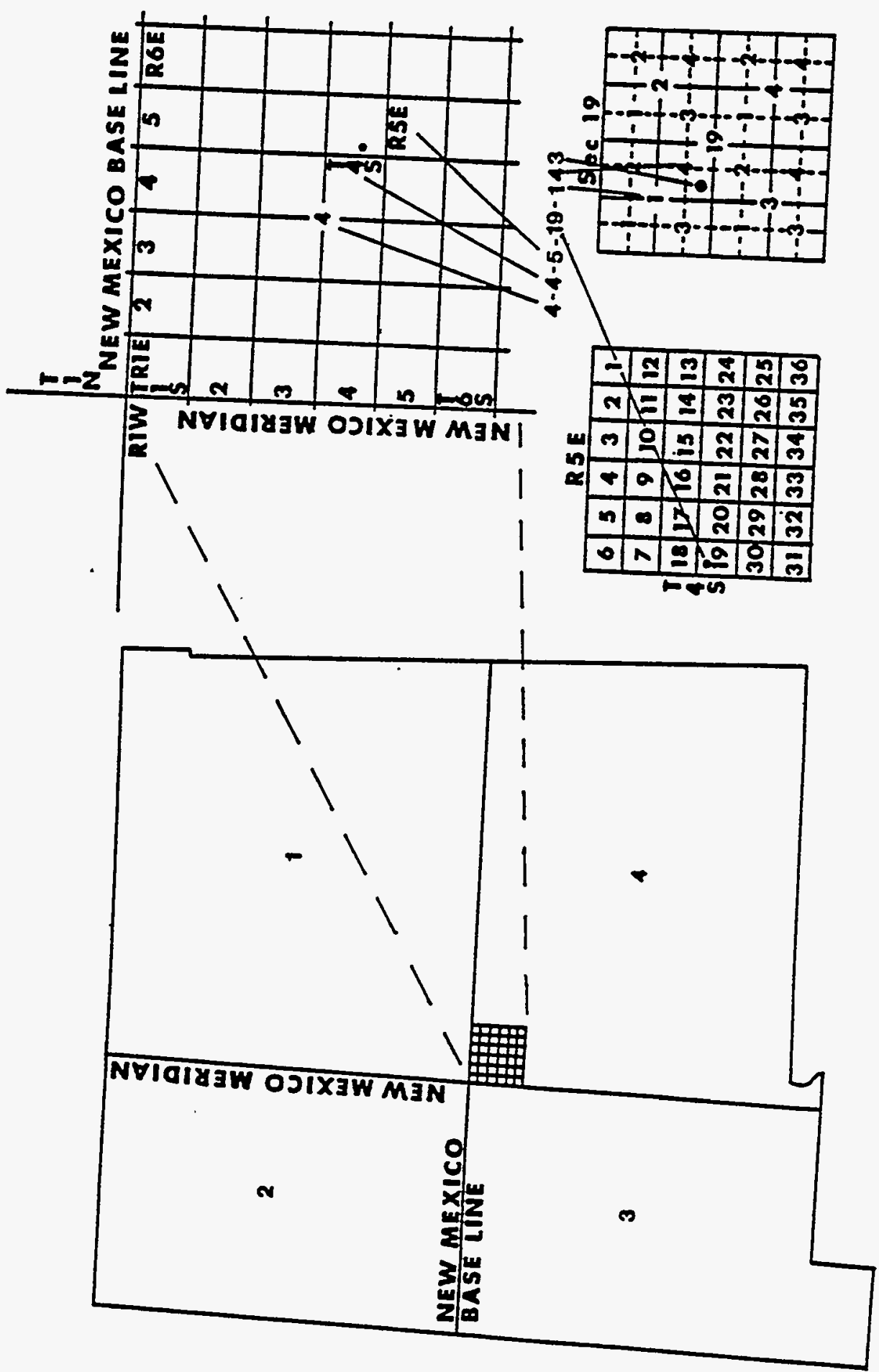

